THE UNIVERSITY OF EDINBURGH

DEPARTMENT OF MECHANICAI ENGINEERING

\title{
A STUDY OF THE PERFORMANCE OF A NONLINEAR RESONANT VIBRATORY CONVEYOR
}

\author{
GEORCE G. IECKIE
}

Thesis Submitted for the degree of Doctor of Philosophy in the University of Edinburgh

Department of Mechanical Engineering 1967.

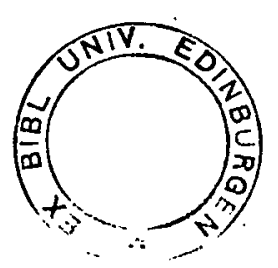




\section{ACKNOWIEDGEMENTS}

This research was carried out under a studentship from the Science Research Council who also gave an equipment grant for further studies. The research was carried out in the Engineering Department of the University of Edinburgh.

The author acknowledges with gratitude the advice of his thesis supervisors, particularly Dr.A. D. S. Barr, present acting head of the department, and Dr. J. D. Robson, formerly of this department and now Professor at Glasgow University.

Special thanks are due to Mr. George Smith for his skill and advice in constructing the apparatus used, Mrs. Marshall for her patience in typing, and Miss Anderson for photographic work. 


\section{SUMMARY}

This thesis investigates the performance of a resonant non-linear vibratory conveyor in which the conveying surface is horizontal and the maximum vertical acceleration is less than the acceleration of gravity. The mechanism by which material transport occurs has been previously established by other workers, and it is known that when the table surface is sinusoidal accurate predictions of transport rate can be made. When the conveyor has a non-linear spring force the displacement waveform can only be calculated with some difficulty.

Two experimental conveyors were constructed. In both an unsymmetric non-linear spring characteristic was formed with a stiff 'impact' spring on one side of the vibrating conveyor. Various stiff springs were used, and the gap between the stiff spring and the conveyor in its equilibrium position was variable. One conveyor was for vibration in a horizontal direction and the other allowed vibration at angles of inclination up to $30^{\circ}$. The exciting force was applied by an electromagnetic vibrator through a force transducer which allowed accurate measurement of the input force. Analogue computer circuits and digital computer programmes were developed to allow comparison between experiment and theory and to investigate the effect of material load on conveyor performance.

It is confirmed that the performance of a linear conveyor is very.. dependent on tuning, and this is seriously affected when the material load is heavy. By contrast a non-linear conveyor is less dependent on accurate control of frequency when the material load is negligible, and gives travel rates which are almost independent of material load over wide ranges. 
Good agreement is found between computer results and experimental results, and it is envisaged that computer techniques would be a valuable aid in conveyor design.

Expressions are developed for the maximum travel rate which could be obtained in terms of maximum conveyor accleration, the period of the vibration and the coefficient of friction between the conveyor surface and the material to be transported. These results could prove the basis for the design of more sophisticated conveyors. 


\section{CONTENTS}

1. Chapter I Introduction ............. I 1.1 Vibratory Conveyors ............. 2

1.2 Review of Previous

1.3 Scope and purpose of research ........ 6

2. Chapter 2 Theoretical Development ........ 9

2.1 Theory of sliding .................. 10

2.2 Introduction of non-linear element ........ II

2.3 Equations of motion ................. 13

2.4 Solution of equations ................... 19

3. Shapter 3 Experimental Procedure ......... 25

3.1 Scope of experiments ............ 25

3.2 Requirements of system ........... 25

3.3 Preliminary investigation .......... 27

3.4 Considerations affecting design ....... 27

3.5 Measurement of force ............ 28

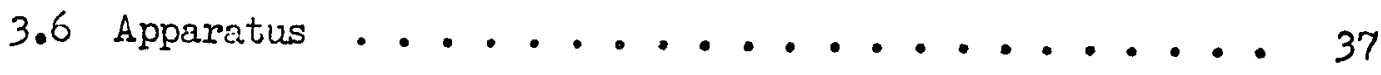

3.7 Procedure ....................... 46

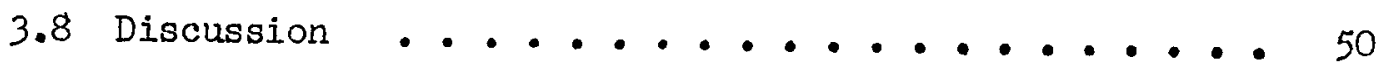

4. Chapter 4 An approach using an Electronic Analogue Computer ........... 52

4.I Introduction ..................... 52

4.2 The 'Minispace' Analogue Computer ........ 52

4.3 Basic computer circuit ............ 53

4.4 The SC 30 and 247 Computers ................ 59

4.5 Operating states of a computer .................. 62 
4.6 Computer circuit for horizontal table motion when slider has negligible mass ........ 64

4.7 Circuit for slider of negligible mass $m$ horizontal table vibrating at angle $\beta \ldots 70$

4.8 Generation of forcing function ........ 72

4.9 Ancillary equipment ............... 73

4.10 Procedure ................... 73

4.11 Circuit for table vibrating horizontally with material mass comparable to table mass ........ 75

4.12 Imperfoctions in analogue circuits ....... 77 4.13 Further circuits .............. 78

5. Chapter 5 Solution using a digital computer ...... 80

5.1 A quick approximate method to find the waveform of a non-linear single degree of freedom system with an unsymmetric bi-linear spring characteristic ....

5.2 Method of solution adopted .......... 85

5.3 Basic computer programme .......... 88

5.4 Computer programme for horizontal table motion with mass being conveyed comparable to table mass ... 91

5.5 Programme extended to inclination of vibration at $\beta^{\circ} .97$

5.6 Further extensions which could be made...... 104 Digital Computer Programme .......... 105

6. Chapter 6 Results and Discussion ......... II3 6.1 Results from System A . . . . . . . . 113 6.2 Results from System B ........... 117 6.3 Further results ostained by computer . . . . . 120 6.4 Plates and additional figures $\ldots . . . . . . . . . .123$ 6.5 Discussion .......................... 124 
7. Chapter 7 Conclusions . . . . . . . . . 135

7.I Objectives of the investigation . . . . . . . I35

7.2 Overall results and conclusions ......... 135

7.3 Factors effecting design of a conveyor . . . . . 137

7.4 Areas for further study . . . . . . . . . 139

Bibliography . . . . . . . . . . . . . 140

Appendix A System Coefficients ........... 144

Appendix B Development of expressions of optimum travel rate for a given maximum acceleration . . . 147

B.1 Case (i) Travel rate resulting from a purely horizontal conveyor motion ...... 148

B.2 Case (ii) Surface motion at angle of inclination $\beta^{\circ}$. 150

B.3 Case (iii) Motion resulting from horizontal and vertical accelerations which are independent .......... 155

B.4 Discussion . . . . . . . . . . . 157

Appendix C Experiments with sand .......... 163

Appendix D Theory of motion when centre of gravity is not at geometric centre ......... 167

Appendix E Ultra-harmonic and ultra-subharmonic response $\quad 171$ 


\section{NOTATION}

Note - see separate notation for digital computer programme on page 105.

\begin{tabular}{|c|c|c|}
\hline$A, B, C$ & Constants & \\
\hline$f, \omega$ & circular frequency of forcing function & $\mathrm{rads} / \mathrm{sec}$ \\
\hline$F(t)$ & applied external force & Ib \\
\hline $\mathrm{g}$ & acceleration of gravity & ins $/ \mathrm{sec}^{2}$ \\
\hline$g$ & gap setting $\left(x_{g}\right)$ & ins \\
\hline$K$ & spring stiffness & $1 \mathrm{~b} / \mathrm{in}$ \\
\hline$M$ & mass of vibrating conveyor & $1 b \cdot \sec ^{2} /$ in \\
\hline $\mathrm{m}$ & mass of material being transported & Ib.sec $\sec ^{2} /$ in \\
\hline $\mathrm{N}$ & normal force & Ib \\
\hline $\mathrm{n}$ & damping factor & $\sec ^{-1}$ \\
\hline $\mathrm{p}$ & natural frequency & rads/sec \\
\hline $\mathrm{R}$ & reaction force & $1 \mathrm{~b}$ \\
\hline$t$ & time & sec \\
\hline $\mathrm{T}$ & period if vibration & sec \\
\hline $\mathrm{U}$ & maximum conveyor acceleration & ins $/ \sec ^{2}$ \\
\hline $\mathrm{U}_{\mathrm{x}}$ & maximum horizontal conveyor acceleration & ins $/ \sec ^{2}$ \\
\hline$U^{*}$ & limiting conveyor acceleration & ins $/ \sec ^{2}$ \\
\hline$X(t), \dot{X}(t), \ddot{X}(t)$ & $\begin{array}{l}\text { displacement, velocity, acceleration of } \\
\text { conveyor }\end{array}$ & $\begin{array}{l}\text { ins, ins/sec, } \\
\text { ins/sec }\end{array}$ \\
\hline$x(t), \dot{x}(t), \ddot{x}(t)$ & $\begin{array}{l}\text { displacement, velocity, acceleration of } \\
\text { material }\end{array}$ & $\begin{array}{l}\text { ins, ins } / \text { sec, } \\
\text { ins } / \sec ^{2}\end{array}$ \\
\hline $\mathrm{X}_{\xi}$ & gap setting & ins \\
\hline Z & amplitude of steady stete response & ins \\
\hline$\alpha$ & phase angle & radians \\
\hline$\beta$ & angle of inclination of vibration & degrees \\
\hline$\gamma$ & damping factor & $\sec ^{-1}$ \\
\hline
\end{tabular}


displacement/cycle

angle of surface inclination

density

coefficient of friction

period of vibration ins

degrees

Ib. $\sec ^{2} /$ in $^{4}$

sec. 


\section{GHAPTER I}

\section{INTRODUCTION}

In recent years much thought and research has been given to methods of transporting material by various types of conveyor. While the conventional belt conveyor has been in use for a great many years, vibratory and shaker conveyors are a comparatively recent development. Belt conveyors are essentially moving platforms with little or no relative motion between the belt and the material being transported, whereas shaker and vibratory conveyors whose motion is oscillatory, convey the material wholly by relative slipping or throwing. This thesis is devoted to vibratory conveyors in which transport occurs wholly by relative slipping.

The difference between shaker conveyors and vibratory conveyors is in the method by which the conveyor displacement is produced. In the former the conveyor motion is caused by direct mechanical drive while the motion of a vibratory conveyor is the response of the system to an applied periodic force. It is noted that much previous research into the performance of vibratory conveyors has been carried out on special small amplitude high frequency shaker conveyors, this allowing interest to be concentrated on the mechanism of sliding and ignore effects of changes in the tuning of the system through damping or material load.

It is however worth digressing to discuss shaker conveyors. These were the earliest form of oscillatory conveyors used, and are still common in screening operations in the coal industry. They are generally large amplitude low-frequency conveyors. The motion is imparted by direct drive from anc electric motor via a cam or eccentric or occasionally by hydraulic or pneumatic drive. Occasionally various 
types of motion are imparted to the conveyor surface by the use of curved guides which produce any desired conveyor displacement, including sharp jerks to improve the performance. While spring mountings or buffers may be used they are not basic to the conveyor motion. Shaker conveyors, unlike vibratory conveyors are characterised by low frequency of operation $1-2 \mathrm{c} / \mathrm{s}$ and large amplitudes $3-9$ ins.

\subsection{Vibratory Conveyors}

The essential features of a vibratory conveyor defined as above are that it should have a rigid conveying surface mounted on springs to which a periodic exciting force is applied. In the most simple case the spring mounting, which has the form of linear coil springs, is inclined at an angle to the horizontal. The exciting force is either applied by rotating out-of-balance weights or by on electro-magnetic vibrator aligned in the same direction as the spring mounting. The conveying surface may thus be considered as the mass of a single-degreeof-freedom Iinear system which, under the influence of an applied periodic exciting force will execute simple harmonic motion on its spring mounting The amplitude of the conveyor motion is dependent on the magnitude of the applied force and its frequency. Such systems are usually tuned to operate near the resonance of the system when large response magnification is obtained. Vibratory conveyors can seldom feed up an incline of more than a few degrees, so most surfaces are either horizontal or are given the advantage of a slight/gradient. Under the effect of the horizontal and vertical components of vibration which material placed on the conveyor experiences, relative inovement occurs. Depending on the magnitude of the maximum vertical acceleration this may take the form of either pure relative slipping or of the material being thrown off the conveyor surface. Thus the material is transported by a series 
of slides or hops along the conveyor surface.

The tro types of motion are found in rather different applications. Throwing is usually found. when a bulk material is to be transported. Because the material is airborne for much of the cycle, hot $t_{\frac{\beta}{7}}$ abrasive materials cause little frictional wear on the conveyor surface. By various arrangements and combinations of conveyors, separation, mixing, or cooling of the material can be done while it is being transported. Vibratory conveyors are usually self cleaning, and several materials could be transported in succession without contamination. The length of such conveyors is usually fairly large, up to about $100 \mathrm{ft}$. , though long distances are usually achieved by a succession of conveyors. Under this type of throwing motion fast transport rates can be achieved.

Pure slipping motion is more restricting on the travel rate which can be achieved than is the case for throwing. However rigid bodies in free flight bounce erratically on making contact with the conveyor surface, and throwing is not suitable where steady feed rates of individual parts is required. Recent interest in sliding motion has largely been for applications in feeding components into automated processes. The conveyors in this type of work are often spiral hopper-type, this being a special case of the more common straight conveyor. The slower travel rates obtained with pure slipping are offset by the degree of control over feed rate and the removal of possible breakage due to bouncing. The more gentle material motion gives 'attitude stability' of the components during transport which is lost in throwing and this makes the use of various selection and alignment operations comparatively simple. If tuning of the system and the exciting force can be maintained, small power input is required. Amplitudes are usually small (less than $\frac{1}{2}$ ) and while $50 \mathrm{c} / \mathrm{s}$ is obviously a particularly convenient frequency at which to excite a 
system, the frequency range $5-25 \mathrm{c} / \mathrm{s}$ is more usual.

1.2 Review of Previous Work.

The earliest oscillatory conveyors are found around the period 1915 - 1925, when shaker conveyors and screens appeared in the coal industry. Vibratory conveyors becane more into the light from about 1940. Much of the basic research appears to have been carried out simultaneously at several centres, and there is much duplication in the published works.

The design of a vibrating conveyor is complicated not only in predicting the travel rate for various materials and conditions, but also in that large inertia forces are set up which have to be isolated from the surroundings. This is usually achieved by coupling the conveyor by its springs to a massive base which is itself elastically coupled to the ground. Most industrial plants are thus two mass systems. However the characteristic of the mounting of the base is such as to make the use of a single degree of freedom representation as described in the previous section, acceptably accurate.

Most of the work on vibratory conveyors have used the simplification of making the conveyor surface execute simple harmonic motion whose amplitude was determined by the throw of an eccentric $(4,6)$. This allows easier presentation of results, and also permits the neglect of changes in performance of the conveyor due to slider mass. The research branches Into two parts depending on whether the notion is throwing or sliding.

It has previously been mentioned that conveyors which depend on throwing are generally only suitable for transporting bulk materials due to impact effects when the material lands on the conveyor. Klockhaus (42) ignoring many of the difficulties produced a much simplified solution for this type of motion. Brickman $(5)$ has extended the theory to account 
for the divergences between simple theory and the observed behaviour of fine powders, by considering the porosity of the powder in evaluating the effect of air resistance on the trajectory. Impact effects, which are still not fully included in theories of throwing restricted Redford's (30) experimental work to maximum vertical accelerations of $1.2 \mathrm{~g}$, above which erratic results were obtained. Schraud $(43)$, evaluating the energy losses in inelastic impact between the material and the conveyor surface, calculated the porver requirement of a system, though without experimental verification.

Recent works into linear conveyors whose transportation is achieved by sliding have been very comprehensive. Booth and McCallion $(4,4 a)$ give formulae and tables by which a quick approximate solution to the travel rate can be found in terms of the coefficient of friction, the frequency of vibration and the amplitude of vibration. Berry $(2,3)$ pursued similar lines, but was more interested in the transportation of a bulk material (grain) than of a single body. All the researches before 1966 made use of a sinusoidal excitation, the horizontal and vertical motions being components of a single displacement, and were, of necessity, in-phase with each other. Redford ${ }^{(30)}$ used two separate sources of excitation and was thus able to vary the phase between the two components. He has shown that out of phase excitation of the components can produce better travel rates with a much smaller dependence on frequency than is the case for in-phase excitation. The Bibliography contains many further references on in-phase linear conveyors.

In the short discussion on shaker conveyors, it was mentioned that these often make use of guides to impart jerks to the conveyor surface and hence cause relative slipping. Gutman $(10)$ indicates that using a 4-bar link to drive a conveyor surface in a purely horizontal direction 
allows transport of the material without the necessity for any gradient. This case discussed by Wolff( ${ }^{(4)}$ does not produce material transport when the conveyor displacement is sinusoidal.

While vibratory conveyors are usually run at or near their resonance frequency in order to achieve large amplitudes for a minimal power requirement, exact tuning is often difficult to maintain, particularly when there are marked fluctuations in the conveyor loading. Kluge ${ }^{(17)}$, discussing this describes a system which has symmetrically placed buffer stops. While these have a slight limiting effect on the amplitude at resonance, the 'backbone' of the response curve is bent slightly forwards and a broader frequency band is available for tuning. Kluge assumed that this small symmetrical non-linearity had no effect on the waveform which remained sinusoidal.

Continuing a project on conventional conveyors using linear springs, Maclartin ${ }^{(21)}$ added a very hard 'limiter' spring at one side of the vibrating table to produce an unsymmetrical non-linear spring characteristic. The advantages expected were a combination of the effects of a shaker conveyor whose motion is non-sinusoidal and an extension of the resonance amplitudes over a much wider frequency band. This report could not be very complete in the short time available, and the object of the present work was to obtain a more comprehensive study of this type of conveyor.

\subsection{Scope and purpose of this research}

This research was undertaken to investigate the performance of a vibratory conveyor whose mounting spring had an unsymmetrical non-linear spring characteristic and to evaluate it against the more usual conveyor with linear restoring springs. Only low-acceleration conveyors were considered, that is, those whose maximum vertical acceleration was less than gravity, so that throwing motion was excluded. The mechanism 
by which slipping takes place has been investigated by other workers, so it was not felt important at this stage, to investigate the effect of changes in parameters such as the coefficient of friction between the material and the conveyor surface. In the case of bulk material where the mechanism of motion is less well established, simple experiments were required to confirm the assumed theories.

The programne of research was as follows:

1) To construct a suitable vibratory conveyor with an unsymmetrical nonlinear resotring spring characteristic which was to be excited in a horizontal direction, the conveyor surface being horizontal. The performance of this system was to be found by exciting it with a periodic force of constant magnitude over the frequency range of the 'non-linear resonance', with various degrees of non-linearity introduced.

2) To construct a similar conveyor to that ahove which would be excited at a variable angle to the horizontal while keeping the conveyor surface horizontal. This system without any 'impact' spring would correspond to the conventional 'linear' conveyor and hence the performance of the system with, and without the non-linear element could be compared.

The ranges over which tests were carried out on the above systems were frequency $5-15 \mathrm{c} / \mathrm{s}$ (Iinear resonance $8-10 \mathrm{c} / \mathrm{s}$ ); Force .75 ib (constant); amplitudes $\rightarrow$ " (not directly controllable); and anglo of inclination of conveyor vibration $0-30^{\circ}$ continuously variable. Only one rigid body (a brass slider) was used and hence variation of the coefficient of friction between table and slider was not considered.

A theoretical approach was required which would, allow prediction of the performance of any given conveyor without recourse to practical experiment. The mechanism by which relative sliding occurs is well established. However the problem was complicated by the need to know 
the response of an extermely non-linear system to which quasi-linear techniques are not applicable. When solving non-linear differential equations in which the non-linearity is in the restoring spring force, analogue computer techniques immediately suggest themselves. Accordingly it was decided to develop an analogue computer circuit to simulate the experimental work.

It was also considered worth while to develop a programme for a digital computer to allow the performance of any given conveyor to be calculated. This was not because the problem was considered to be more suited to a digital computer than to analogue methods. Indeed the opposite was the case. However, outside universities and similar research institutes access is more readily available to large digital machines than to even mediurn sized analogue computers. In this research project research facilities were somewhat limited and the analogue computer had only 54 amplifiers. It was found possible to extend the digital programme to cases such as a heavy weight on a conveyor vibrating in an inclined plane; which were not possible by other methods.

It was obviously desirable to find exact solutions analytically and to explain any phenomona found. The preoccupation of research in the field of non-linear dynamics with the concept of quasi-linear systems offers little help for dealing with systems whose non-linearity is extreme, and it became obvious that analytic methods for such systems seldom exist. When designing an oscillating conveyor it is possible that the maximum acceleration to which the table is subjected would be an important factor. It was found possible to establish absolute maxima for the travel rate which can be obtained from a given maximum acceleration and coefficient of friction. These form an important basis for evaluating the efficiency of any conveyor displacement waveform. 


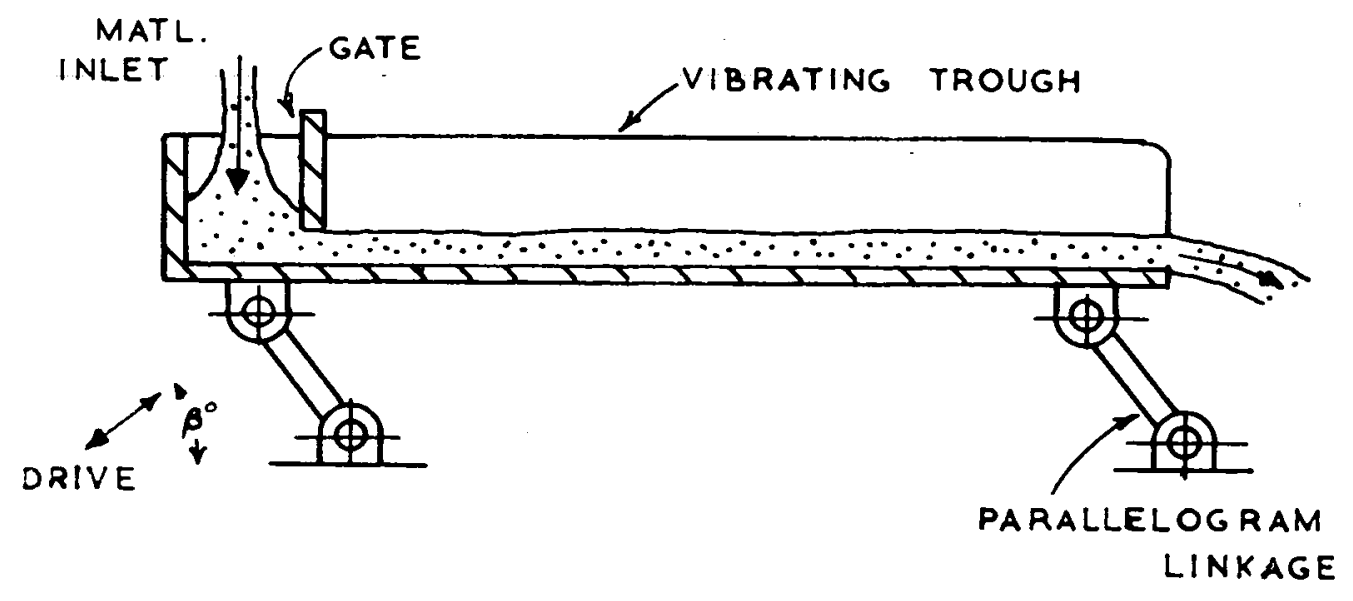

(A) Elementary Linear System

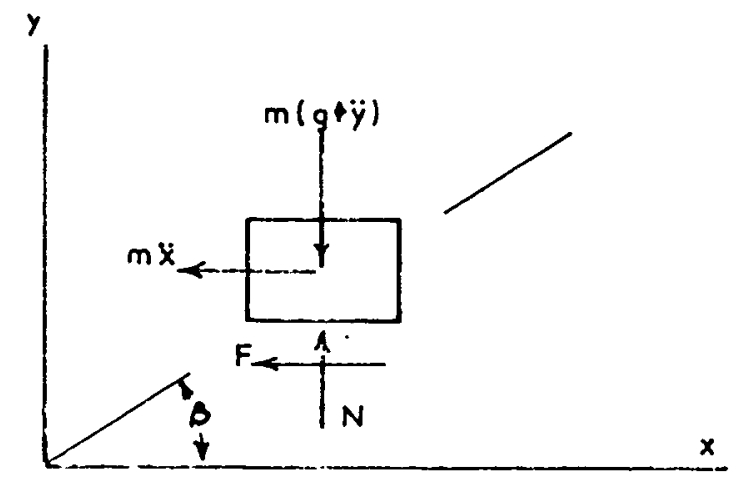

(B) Forces on Moterial

FIG. $2: 1$ 


\section{CHAPTER 2}

\section{THEORETICAL DEVELOPMENT}

Vibratory conveyors in which the surface motion is sinusoidal have been in use for some 30 years, and the analysis of the mechanism by which transport occurs has been thoroughly carried out. The usual model studied, and indeed the one most commonly used in industry is shown in Fig. 2.10. The conveyor vibrates in a straight line motion at an angle $\beta^{\circ}$ to the horizontal. Various systems have been used to produce the motion from a mechanical linkage to the excitation of a linear single degree of freedom system. Most investigators have not discussed the system used, being interested in the resulting amplitudes rather than how they were obtained.

The material transported is usually idealised. Bulk materials are assumed to move as a rigid body, the coefficient of friction between the material and the conveyor surface is assumed to have the same value throughout a cycle, and the effect of the relative motion of the material on the conveyor motion is neglected. The former two assumptions have been shown to be valid, at least within the limits of the experiments conducted. The latter approximation, that of neglecting the mass effect of the material is demonstrably wrong. In cases where the conveyor forms the mass of a linear single degree of freedom spring/mass system, the response to an exciting force depends critically on the vibrating mass of the system.

On the other hand it can be seen that where the motion is prescribed by a mechanical linkage the conveyor displacement is governed principally by the exciting system. This is certainly true when a large motor, with possibly a flywheel, is used such that the alternating loads do not 

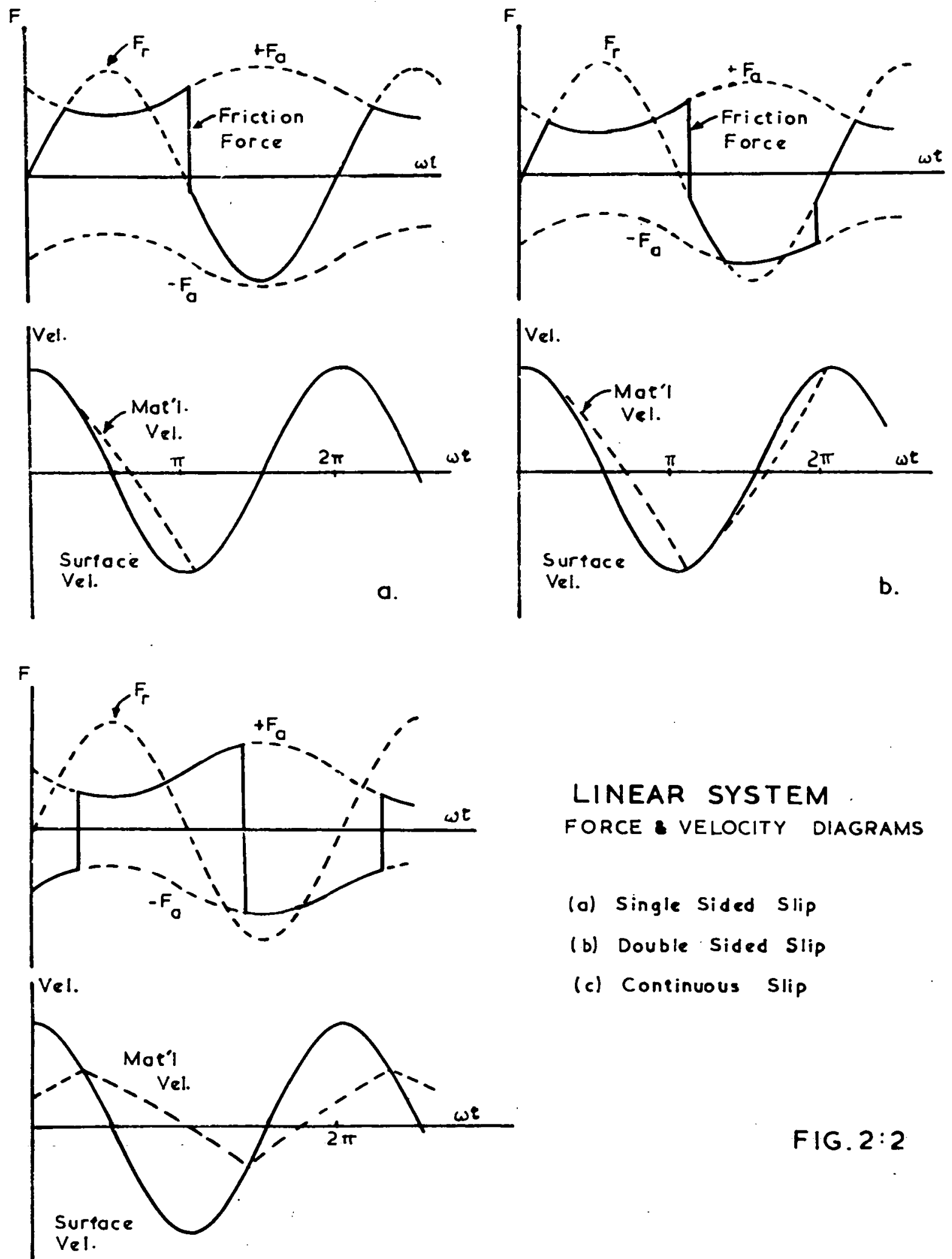

LINEAR SYSTEM

FORCE \& VELOCITY DIAGRAMS

(a) Single Sided Slip

(b) Double Sided Slip

(c) Continuous Slip

c. 
produce speed changes. In the present investigation resonant systems only are considered, when the assumption of negligible material mass is not valid.

\subsection{The theory of sliding}

Figure 2.2 shows force and velocity diagrams for the conveyor surface and material for a linear system. It will be familiar to all who have read any of the literature on vibratory transport as the waveforms shown are the basis of the theoretical analysis.

Considering the forces acting on the material transported Fig. 2.1b the normal reaction between the material and the surface is $N=m(g+\ddot{y})$ where $m$ is the mass of the material and $\ddot{y}$ the vertical acceleration. The limiting horizontal force between the material and the surface is $\mu \mathrm{N}$ where $\mu$ is the coefficient of friction. There is a horizontal inertia force on the material $m \ddot{x}$ where $\ddot{x}$ is the horizontal component of acceleration. When this exceeds the limiting value slipping will start. Equating the horizontal forces on the material gives its equations of motion during slipping

$$
m \ddot{x}=\mu \mathrm{m}(g+\ddot{y}) \operatorname{sign}(\dot{x}-\dot{x}) \quad \ldots 2.1
$$

$(\dot{\mathrm{X}}-\dot{\mathrm{X}})$ is the relative velocity between the table and the material. When $\dot{x}=\dot{X}$ the friction force is indeterminate and may have any value between $\pm \mu \mathrm{m}(\mathrm{g}+\ddot{\mathrm{y}})$. Figures 2.2 show the available friction force between the surface and the material $F_{a}= \pm \mu m(g+\ddot{y})$ and the required force if slipping is not to occur $F_{r}=m \ddot{x}$. Then $F_{r}=F_{a}$ is the limiting condition for slipping to start.

The classification of the slipping motion into the three types shown in Fig. 2.2 is standard. The types show the progression as $\mu$ is decreased all other parameters remaining constant. In the case of 

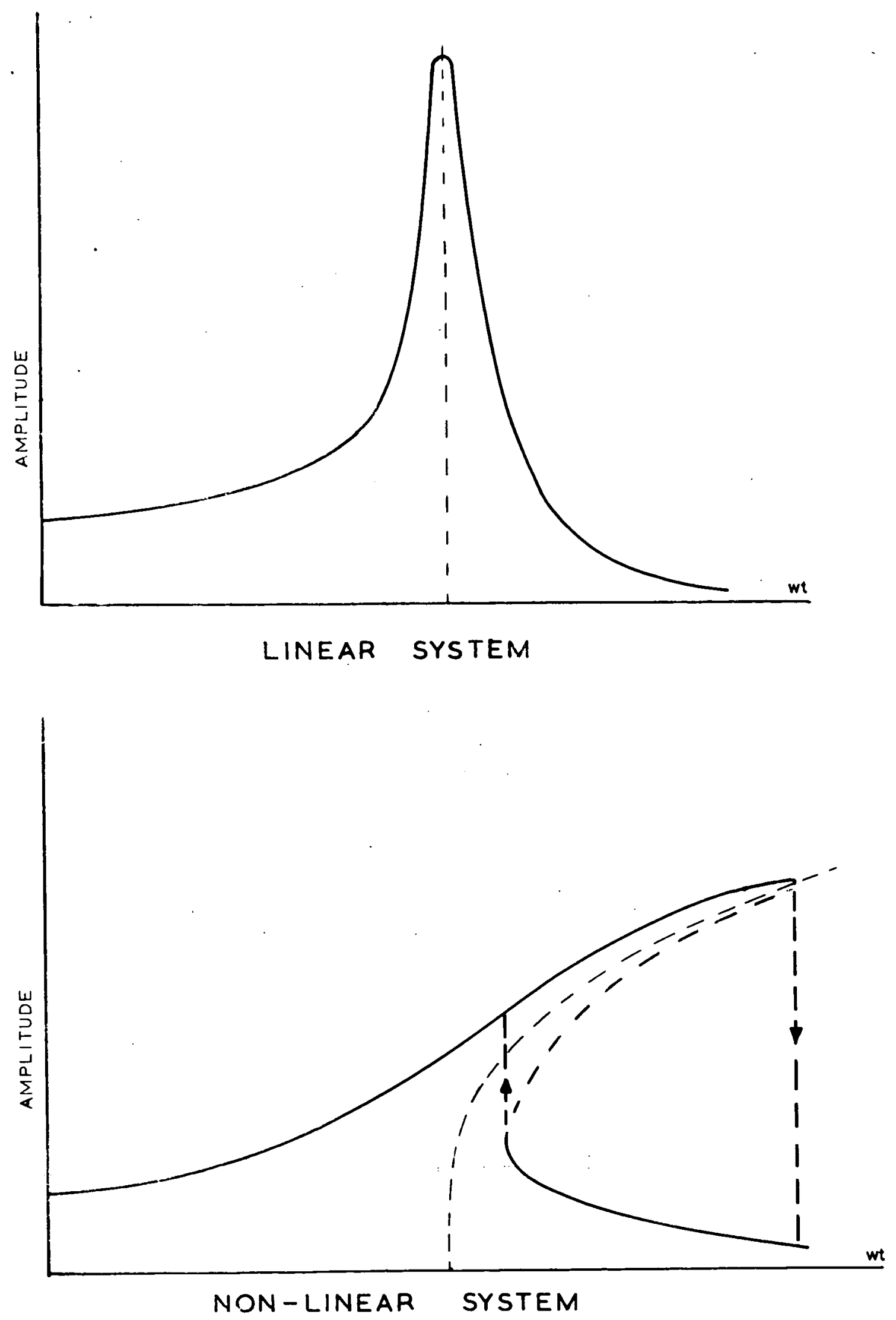

FIG. $2: 3$ 
single sided slip (forward only) $\mathrm{F}_{r}>\mathrm{F}_{\mathrm{a}}$ only over one part of the cycle. As $\mu$, and hence $F_{a}$, is reduced there are two regions of the cycle in which $\left|F_{r}\right|>\left|F_{a}\right|$, but there is still a period of no relative slip between the regions of forward and beckward relative velocity (double sided slip). With $\mu$ reduced still further a continuous slipping motion occurs with the direction of relative velocity changing instantaneously each time $\dot{\mathrm{x}}=\dot{\mathrm{x}}$.

In the linear system when the surface motion is sinusoidal, all surface displacements, velocities and accelerations are known functions of time, and equations can be solved to give values of $\omega t$ at the boundaries of each region of slipping. The calculations become excessively tedious in the case of continuous slipping; Booth and McCallion ( 4 ) have developed a programme for a digital computer to solve this motion. Having found the slider and table velocities the relative displacement per cycle is obtained by integrating the relative velocity. In the case of sinusoidal displacements the results can be tabulated in non-dimensional form using the table acceleration as a variable rather than the more basic elements of the resonant system from which the response was obtained.

\subsection{Introduction of non-linear element}

The most usual industrial system is the linear spring/mass model, the excitation being applied by rotating out of balance weights or by an electro-magnetic vibrator. Fig. 2.3a shows the response curve of a linear system to an exciting force $A \sin \omega t$. The sharp nature of the resonance curve (damping would obviously be minimised for power economy) shows that fairly exact tuning in necessary if amplitudes are to be maintained approximately constant near resonance. This is always difficult to achieve, small frequency changes in the exciter or 


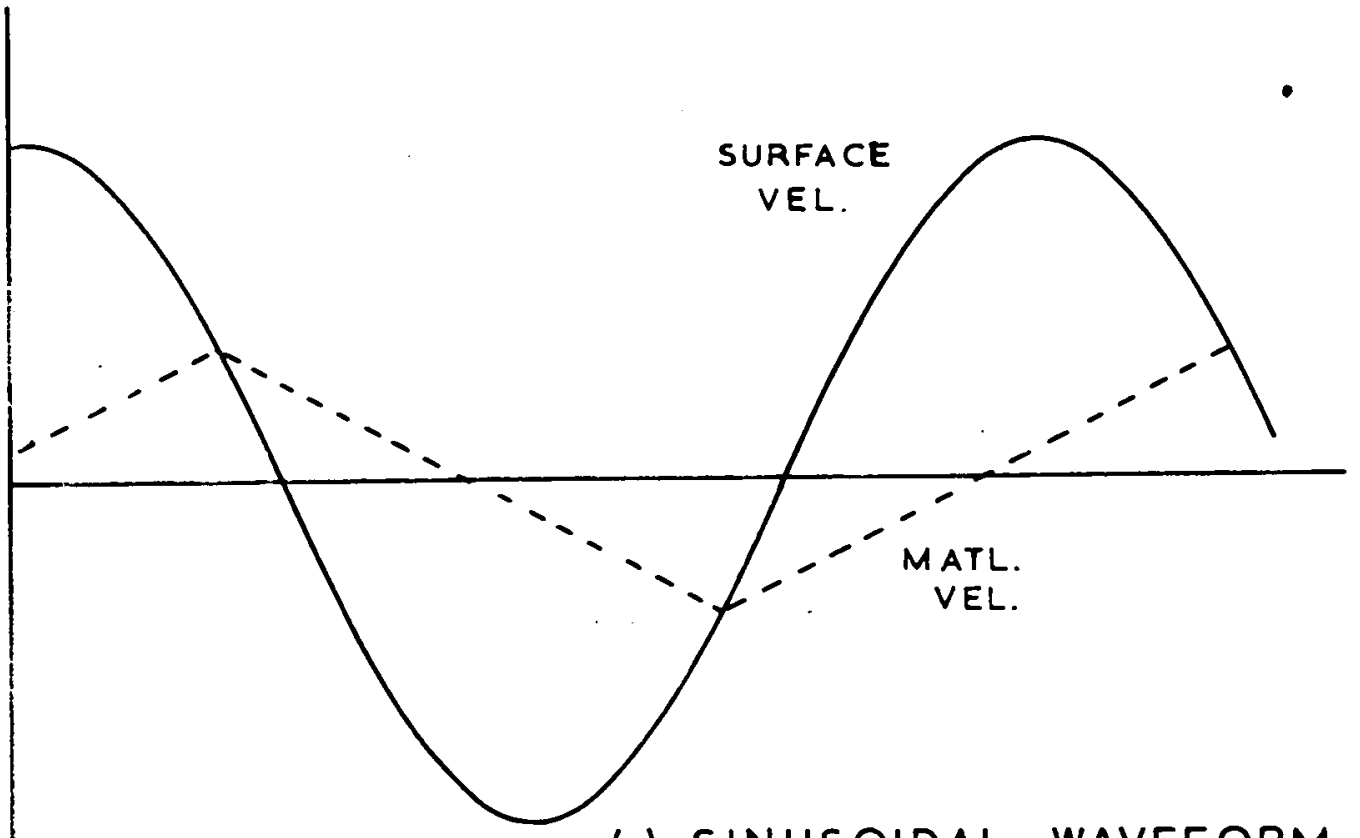

(a) SINUSOIDAL WAVEFORM

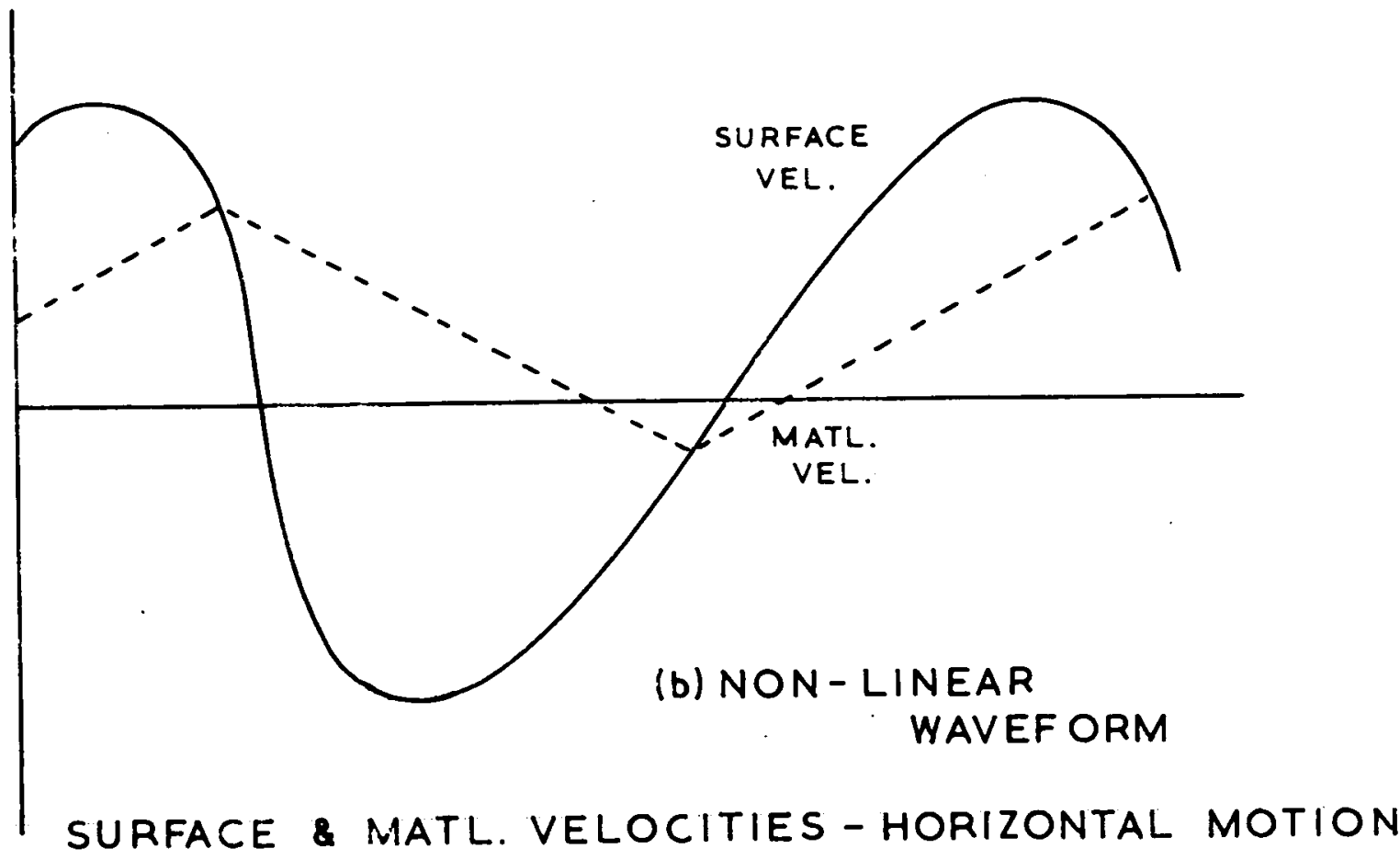

FIG. 2:4 
or variations in the spring characteristic being more or less inevitable. However, since the vibrating mass is a function of the material load, and damping is unlikely to remain constant in use, some de-tuning must occur.

Fig. 2.3b shows the response curve of a system similar to the one above when a non-linear term is added to the spring characteristic. It is obvious that over much of the curve small changes in the frequency of excitation produce little effect on the amplitude of the response. Further, changing damping in a non-linear system of this type changes the 'jump' frequencies, but has less effect on the amplitude of response at other points.

Considering Fig. 2.4a which is a special case of Fig. 2.2 c when the surface is vibrating in the horizontal plane with no vertical component such that $\mathrm{F}_{\mathrm{a}}= \pm \mu \mathrm{mg}$, it can be seen that due to symmetry of the waveform there is no nett displacement per cycle. This is true for any symetrical waveform. If however, the velocity waveform can be made unsymmetrical such as is shown in Fig. $2.4 \mathrm{~b}$ material transport will occur without the aid of a vertical component.

It was thus decided to develop a non-linear system with an unsymmetrical non-linear spring characteristic. The bi-linear characteristic of Fig. 2.5 is particularly convenient to produce and to analyse. The restoring force

$$
\begin{array}{rlrl}
k(x) & =K_{1} X & x \leqslant x_{g} & \\
& =K_{1} X_{g}+K_{2}\left(x-x_{g}\right) & x \geqslant x_{g} \quad \ldots 2.2
\end{array}
$$

can be represented by a power expansion

$$
k(x)=k_{1} x+k_{2} x^{2}+k_{3} x^{3} \ldots \quad \ldots 2 \cdot 3
$$


Powers at least as high as the fifth in $\mathrm{x}$ are required to give a good approximation. Analysis of systems of this order is not usually possible.

When discussing un-symmetrical non-linear systems it becomes meaningless to talk about the maximum amplitudes of the resulting motion, as the maximum displacement on the 'hard' side will not be the same as that on the 'soft' side. The motion could be represented by a Fourier expansion of which each component frequency term would have an amplitude in the usual sense.

\subsection{Equations of motion}

Assuming for the moment that expressions for acceleration, velocity and displacement for the conveyor can be found, the theory of sliding developed at the start of this chapter applies equally to linear or to non-linear systems.

\subsubsection{Material idealised as weightless particle.}

The most simple case is that of the table vibrating in the horizontal plane, there being no vertical component, and a material or 'slider' being transported whose mass is negligible. Then the table motion is described by

$$
\ddot{M} \dot{X}+C \dot{X}+k(X)=F(t)
$$$$
\ldots 2.4
$$

and the slider motion is
i) no slipping
$\dot{\mathrm{x}}=\dot{\mathrm{X}}$
ii) slipping$$
\ddot{n}=\mu \mathrm{mg} \cdot \operatorname{sign}(\dot{\mathrm{X}}-\dot{\mathrm{x}})
$$$$
\ldots 2.5
$$

Slipping starts from the condition $\dot{x}=\dot{x}$ if $|\ddot{x}|>\mu g$ and lasts until $\dot{x}=\dot{x}$, when, depending on the magnitude of the acceleration at that particular instant, the direction of slip may be reversed or else a period of no relative slip entered. The slider velocity and displacement may be obtained by integrating the second of equations 2.5 . 


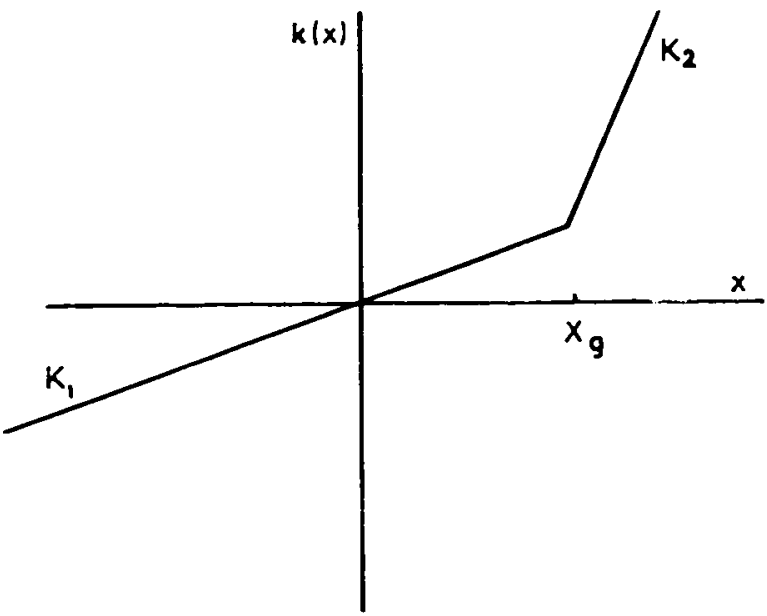

Bilinear Spring Characteristic

FIG. $2: 5$

Table \& Surface Horizontal
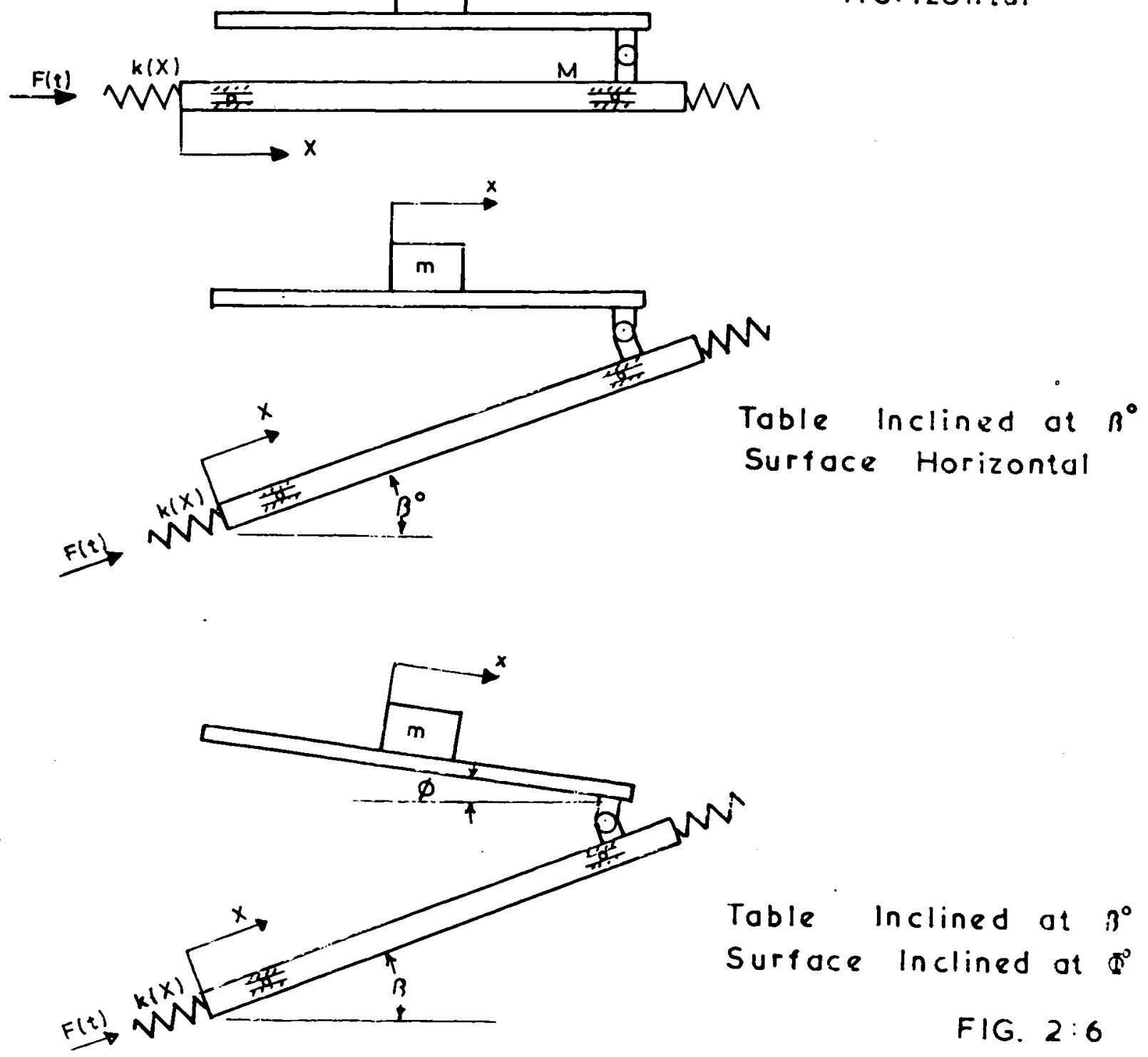

Table Inclined ot $3^{\circ}$ Surface Inclined of $\Phi^{\circ}$

FIG. $2: 6$ 
Constants of integration are found from the table motion at the instant $t_{0}$ when slip started. This gives velocity as a linear function of time during slipping.

In a similar way equations of motion can be derived for the above case with the table vibrating in a plane at $\beta^{\circ}$ to the horizontal, but with the conveying surface horizontal. For the conveyor

$$
\ddot{M}=F(t)-C \dot{X}-k(X)-M g \sin \beta
$$

where $X$ is defined along an axis at $\beta^{\circ}$ to the horizontal. The last term in the equation is the effect of the weight of the table causing a new equilibrium position to be set. For a bilinear system defined by equation 2.2 with $x_{g} \geqslant 0$ this term causes a shift of $-\frac{M_{g}}{K_{I}} \sin \beta$. If $X_{g}$ is measured from this new equilibrium condition, as would be the case in a physical system the equation can be re-written

$$
\ddot{M} \dot{X}^{\prime}+\dot{C X}^{\prime}+k\left(X^{\prime}\right)=F(t)
$$

where

$$
x^{\prime}=x+\frac{M g \sin \beta}{K_{1}}
$$

which is identical in form to equation 2.4. In this case the slider motion is found from

i) no slipping $\quad \dot{\mathrm{x}}=\dot{\mathrm{x}} \cos \beta$

ii) slipping $\quad \quad \ddot{x}=\mu m(g+\ddot{x} \sin \beta) \cdot \operatorname{sign}(\dot{x} \cos \beta-\dot{x}) \quad \ldots 2.7$

Slipping starts from the condition $\dot{x}=\dot{x} \cos \beta$ if $|\ddot{x} \cos \beta|>\mu(g+\ddot{x} \sin \beta)$ and continues until $\dot{x}=\dot{x} \cos \beta$ as before. The slider velocity cannot be found by integrating the second of equations 2.7 unless $X$, the table acceleration, is known.

This latter case can be extended to include the case when the conveying surface is not horizontal but inclined at some angle $\phi$. Considering the case when the slope is 'downhill' (Fig. 2.6c) the slider motion is given by 
i) no slipping $\quad \dot{\mathrm{x}}=\dot{\mathrm{X}} \cos (\beta+\phi)$

ii) slipping $\quad m \ddot{x}=\mu R \cdot \operatorname{sign}(X \cos (\beta+\phi)-\dot{x})+m g \sin \phi$

where $\mathrm{R}=\mathrm{mg} \cos \phi+m \ddot{x} \sin (\beta+\phi) \quad \ldots 2.8$

and the table motion is still given by equations 2.6. Slipping will continue until $\dot{x}=\dot{x} \cos (\beta+\phi)$. On this equation, as for eq. 2.7, the slider velocity cannot be obtained by integration unless the table acceleration is known.

2.3.2 Material has a mass comparable to that of the table.

In conveying a bulk material such as gravel in a trough shaped conveyor with a depth of several inches of material, it is likely that the load of material on the trough at any time will weigh more than the trough itself.

Consider the case of a horizontal conveyor vibrating with mass $\mathrm{m}$ on it, the conyeyor motion being restricted to the horizontal. Then when this material is not slipping there is an effective vibrating mass $(M+m)$. When slipping is taking place the conveyor experience a dry coulomb friction force of $\pm \mu \mathrm{mg}$. The equations describing the table motion are

i) no slipping $(M+m) \ddot{X}+C \dot{X}+k(X)=F(t)$

ii) slipping $\quad \ddot{M}+C \dot{X}+\mu m g . \operatorname{sign}(\dot{X}-\dot{x})+k(X)=f(t) a n$ a $\ldots 2.9$

The equations of slider motion are as for (2.5) viz.

$$
\begin{array}{ll}
\text { no slipping } & \dot{x}=\dot{X} \\
\text { slipping } & \ddot{m} x=\mu \mathrm{mg} \cdot \operatorname{sign}(\dot{x}-\dot{x})
\end{array}
$$

In the case of continuous slip only the second of equations 2.9 applies, and the effect of the material mass is to introduce a Coulomb damping force. Coulomb damping is, of course, a non-linear effect, having more effect on the system when amplitudes are small than when large. This indicates that when the larger amplitudes associated with resonance are involved one would expect the most important changes in the non-linear case to be in the frequency at which the 'jumps' occur. In the linear 
case damping is much more important than in the non-linear case. When single or double sided slip occurs the acceleration $\ddot{X}$ may be discontinuous. When slipping starts $m \ddot{X}=\mu m g . s i g n(\dot{X}-\dot{x})$ and no discontinuity occurs. After slipping, with $\dot{x}=\dot{x}$, the table acceleration will have a different value than when slipping started, so the switch from the second of equations 2.9 to the first will produce a jump in acceleration.

This system can be further developed to include the case when the table is vibrating at $\beta^{\circ}$ to the horizontal with the conveying surface horizontal. If no relative slip occurs then the table motion is given by $(M+m) \ddot{X}+C \dot{X}+k(X)=F(t)-(M+m) g \sin \beta$

If, as before, the spring characteristic is defined with reference to the equilibrium position of the table under its own weight with no added material then a new origin can be used with $X^{\prime}=X+\frac{M g \sin \beta}{K_{I}}$. Then, for no slipping

$$
(M+m) \ddot{X}^{\prime}+C \dot{X} 1+k\left(X^{\prime}\right)=F(t)-m g \sin \beta \quad \ldots 2.10
$$

When slipping occurs

$$
\begin{gathered}
M " x=F(t)-C \dot{X}-K(X)-M g \sin \beta-m(g+\ddot{X} \sin \beta) \sin \beta-\mu m(g+\ddot{X} \sin \beta) \cos \beta \cdot \operatorname{sign}(\dot{X} \cos \beta-\dot{X}) \\
\text { or }\left\{M+m \sin ^{2} \beta+\mu m \sin \beta \cos \beta \cdot \operatorname{sign}(\dot{X} \cos \beta-\dot{X})\right\} \ddot{X}+C \dot{X}+k(X) \\
=F(t)-(M+m) g \sin \beta-\mu m g \cos \beta \cdot \operatorname{sign}(\dot{X} \cos \beta-\dot{X})
\end{gathered}
$$

and using the above change of origin gives

$$
\begin{aligned}
\left\{M+m \sin ^{2} \beta+\mu m \sin \beta \cos \beta \cdot \operatorname{sign}\left(\dot{X}^{\prime} \cos \beta-\dot{X}\right)\right\} \ddot{X}^{\prime}+C \dot{X}^{\prime}+k\left(X^{\prime}\right) \\
\quad=F(t)-m g \sin \beta-\mu m g \cos \beta \cdot \operatorname{sign}\left(\dot{X}^{\prime} \cos \beta-\dot{x}\right) \ldots 2.11
\end{aligned}
$$

The equations of motion of the slider are given by equations 2.7 viz.

no slipping $\quad \dot{\mathrm{x}} \neq \dot{\mathrm{X}} \cos \beta$

slipping

$$
\ddot{m} \ddot{x}=\mu m(g+\ddot{x} \sin \beta) \cdot \operatorname{sign}(\dot{x} \cos \beta-\dot{x})
$$

Unlike equation 2.9 , equation 2.11 has a change in inertia term as well as a coulomb damping force when the motion is one of continuous slip, and it is difficult to make any prediction about the effect of the added mass on the system. The term in $\mathrm{mg} \sin \beta$ in equation 2.11 is a static 
load which produces a new equilibrium position. Since $x_{g}$ in the bilinear characteristic is set without this term, its effect will be to increase $\mathrm{X}_{\mathrm{g}}$. This, in effect, reduces the non-linearity of the spring and with it the 'jump' frequency.

2.3.3. Throwing - material idealised as particle of negligible weight compared with table. Maximum vertical table acceleration greater than gravity.

Strictly speaking this case is outwith the declared scope of the project. Nevertheless it is an aspect which is covered by many other workers and is briefly included here.

If the maximum vertical acceleration of the conveyor surface is less than $-g$ where $g$ is the acceleration of gravity, then the material on the conveyor is thrown off the surface. When in 'free flight' the vertical motion of the material is given by

$$
\begin{aligned}
& \ddot{y}=-g, \text { giving } \\
& y=-\frac{1}{2} g t^{2}+\dot{x}\left(t_{0}\right) \sin \beta \cdot t+x\left(t_{0}\right) \operatorname{sin\beta } \quad \ldots 2.12
\end{aligned}
$$

where $t$ is measured from $t_{0}$ defined by $\ddot{x}\left(t_{0}\right) \sin \beta=-g$. $\quad \dot{x}\left(t_{0}\right)$ and $X\left(t_{0}\right)$ are the surface velocity and displacement at $t_{0}$. The material regains contact with the surface at $t_{I}$ when $y\left(t_{I}-t_{0}\right)=x\left(t_{I}\right) \sin \beta$. Usually the material is slipping relative to the surface when it is thrown off i.e.

$$
|\ddot{X} \cos \beta|>\mu(g+\ddot{x} \sin \beta) \text { before } \ddot{x} \sin \beta<-g
$$

During the period in which the material is not in contact with the table surface its horizontal velocity is given by $\dot{x}=\dot{x}\left(t_{0}\right) \cos \beta$. Otherwise it is given by equations 2.7 .

This is a much simplified approach which can only be used to cover the case of a material of negligible mass which has a completely inelastic impact with the surface. More accurate investigations show, for example, that equation 2.12 is not valid for fine powders which 
tend to adhere to each other giving an air damping effect. Various assumptions of the nature of impact can be made. When throwing occurs the sliding motion associated with it is relatively unimportant as far as the transport velocity is concerned. It is often stated that steady solutions require that the material be in flight for less than one period of the motion, but this is too restrictive a condition. A fuller study will be found in the references quoted. $(5)$

\subsubsection{Applicability of equations}

All the equations developed in section 2.3 apply to any single degree of freedom spring/mass system whose spring characteristic is a function of displacement. Only viscous damping has been considered in the system. The change of origin carried out is only meaningfully applied to the bilinear spring characteristic defined in equation 2.2, but all other expressions are completely general and in the simplest case cover the usual linear spring.

When the system being investigated is linear and the matericl being transported is assumed to be of relatively negligible mass, then the conveyor motion is simply Asinwt. The velocity and acceleration are easily found. Thereafter to find values of $\omega t$ at the boundaries of forward and backward relative slip is no more than elementary substitution and tedious calculation. If $\mathrm{A}$ is used as a parameter, rather than the more basic system coefficients then the results may be represented in non-dimensional form.

The introduction of non linearities into the system greatly complicates the solution of the differential equations, whether the nonlinear term is from a spring characteristic or the result of coulomb damping or inertia terms from a material of finite weight. This is particularly so when the equation of motion of the material requires 
requires that the conveyor acceleration be known.

\subsection{Solution of the equations}

\subsubsection{The solution if $k(x)$ is linear.}

It has already been indicated that when $k(x)$ is a linear function of $X$ and the material is idealised as a massless particle, no great difficulty attaches to obtaining the travel rate. The displacement, velocity and acceleration of the conveyor are all sinusoidal, and only one equation governs the conveyor motion over the cycle. The relative movement of the material does not affect the conveyor performance. There are a sufficient number of equations to obtain all the boundaries of the regions of relative slipping, and while the solution is tedious it is not complicated.

When the material has a mass which is comparable to that of the conveyor so that it cannot be neglected, up to three equations may be required to describe the table motion over a single cycle. The regions over which each equation is valid are partly defined by the material velocity which is, in turn, a function of the conveyor acceleration, so, more exactly, three pairs of equations are required, for the material motion is inseperable from that of the conveyor. It may be assumed that the conveyor motion will not be sinusoidal. The most usual procedure then would be to represent it by a Fourier series. However, each region is covered by a linear differential equation whose exact solution is known, and is of the form

$$
X(t)=e^{-n t}(A \cos p t+B \sin p t)+Z \sin (f t-\alpha)+C \quad \ldots 2.13
$$

where all the symbols have their usual meaning. When each region is represented by its exact solution with $A$ and $B$ chosen to give continuity of displacement and velocity an exact periodic solution may eventually 
be obtained. This can be done either by adjusting the initial condition at the start of a cycle to give a periodic solution, or by continuing the calculation over several cycles until a periodic solution is obtained. The latter seems the most profitable line to follow since when more than one discontinuity occurs within a cycle there may be no convenient approach to allow improved successive approximations to an initial assumption. The system of equations may be considered as being equivalent to a nonlinearity. That being so the possibility of non-linear phenomena must be considered. Only periodic, harmonic solutions were, in fact, obtained during the course of the investigation.

\subsubsection{The solution when $k(x)$ is non-linear.}

It is not proposed to review all the possible methods of solution of differential equations with non-linear restoring forces. The complexities described in the provious section (2.4.1) for the linear system when several pairs of equations are required to describe the table motion over a cycle apply equally to the non-linear system. This problem is outwith the normal bounds of non-linear mechanics where there is usually a restriction to small non-linearities.

The most simple differential equation involved is

$$
\ddot{M X}+C \dot{X}+k(X)=F(t)=F \sin \omega t
$$$$
\text { ... } 2.14
$$

This is a very common equation in non-linear vibrations. It has solutions $X(t)$ which are uniquely determined once the values of $\dot{X}(0)$ and $X(0)$, the initial conditions at $t=0$, are given. Solutions other than periodic solutions may exist, but these are not usually considered. Solutions may be periodic with period $\frac{m}{n} \cdot \frac{2 \pi}{\omega}$ where $m$ and $n$ are integers. The solutions are classed as harmonic if $m=n=1$; as ultra harmonic if $m=1, n>1$; as subharmonic if $m>1 ; n=1$; and as ultra-subharmonic if $\mathrm{m}$ and $\mathrm{n}$ are mutually prime $\mathrm{m}>1, \mathrm{n}>1$. 
Intuitively one can gain an idea of how subharmonic and ultrasubharmonic resonance occur. If one of the subharmonics has period $\frac{m}{n} \cdot \frac{2 \pi}{\omega}$ which is close to the period of free oscillation of the system, then an oscillation of this particular frequency will be singled out relative to the other subharmonics. However this approach does not advance the solution very far. The problem splits into two parts, proof of existence of a subharmonic, and proof of its stability, the latter being the more difficult. In practical systems results are not found for all values of $m$ and $n$.

Equation 2.14 is usually found in the form of Duffing's equation, $k(x)=p^{2}\left(x+\beta x^{3}\right)$ with $\beta$ small, not so much, one suspects, because it represents a physical problem as because approximate solutions to harmonic and sub-harmonic response are conveniently obtained. A great many analytic methods depend on the condition that the non-linearity is small to obtain convergence of successive approximations. In the physical system being investigated in this work, the non-linear spring characteristic was very marked, and methods developed for quasi-linear systems are clearly not permissible.

From a vast and confusing literature, three approaches seemed at first inspection to merit further consideration. These were found in Hayashi ${ }^{(12)}$, Mitropolsky ${ }^{(25)}$ and Kauscher ${ }^{(29)}$. The latter two solutions can use a bilinear characteristic such as equation 2.2 whereas the former requires that the characteristic be a power expansion equation 2.3. Hayashi considers spring characteristics of $k(X)=a X+b X^{2}+c X^{3}$. The 'required' solution, whether harmonic or subharmonic, is inserted into the equation and the unknow amplitudes found by equating coefficients. Having found the solution its stability is investigated in the form of Hill's equation. The method suffers from the disadvantages that (a) 
the form of the solution must be assumed, (b) the calculations are exhaustive and (c) during the calculation new parameters are introduced. The solution obtained involves these parameters and this tends to lose its physical meaning.

Mitropolsky develops an asymptotic expansion to cover the case of a spring force composed of rectilinear sections. The method allows an accurate solution but involves tedious calculation. (Nor is its value enhanced by the poor quality and inaccurate English translation). Mitropolsky does not continue to the investigation of stability nor the presence of subharmonics.

Rauscher's numerical method is well known. In it, amplitudes are assumed and a corresponding frequency obtained. It gives no indication of stability. The method developed by Brock ${ }^{(6)}$ which is an extension of Rauscher's method was not applicable.

Exact solutions have been obtained to the case of a symmetric spring characteristic composed of rectilinear sections by Den Hartog (II) Not only does this entail exhaustive calculation but it is not applicable to the unsymmetric case. Recent interest in non-linear elements in control systems has produced some new techniques, particularly where stability criterion are concerned. Many of the assumptions which are valid in control systems are not so in vibrations work and these methods are seldom applicable.

2.4 .3 The solution when $K(X)$ is the bilinear characteristic of equation (2.2)

From the discussion above it will be seen that solution of equation 2.14 is not easy if $\mathrm{K}(\mathrm{X})$ is anything more complicated than Duffing's equation, and that the bilinear nature of the spring characteristic hardly helps, at least so far as analytic methods are concerned. It does, however, allow solution in a manner similar to that discussed 

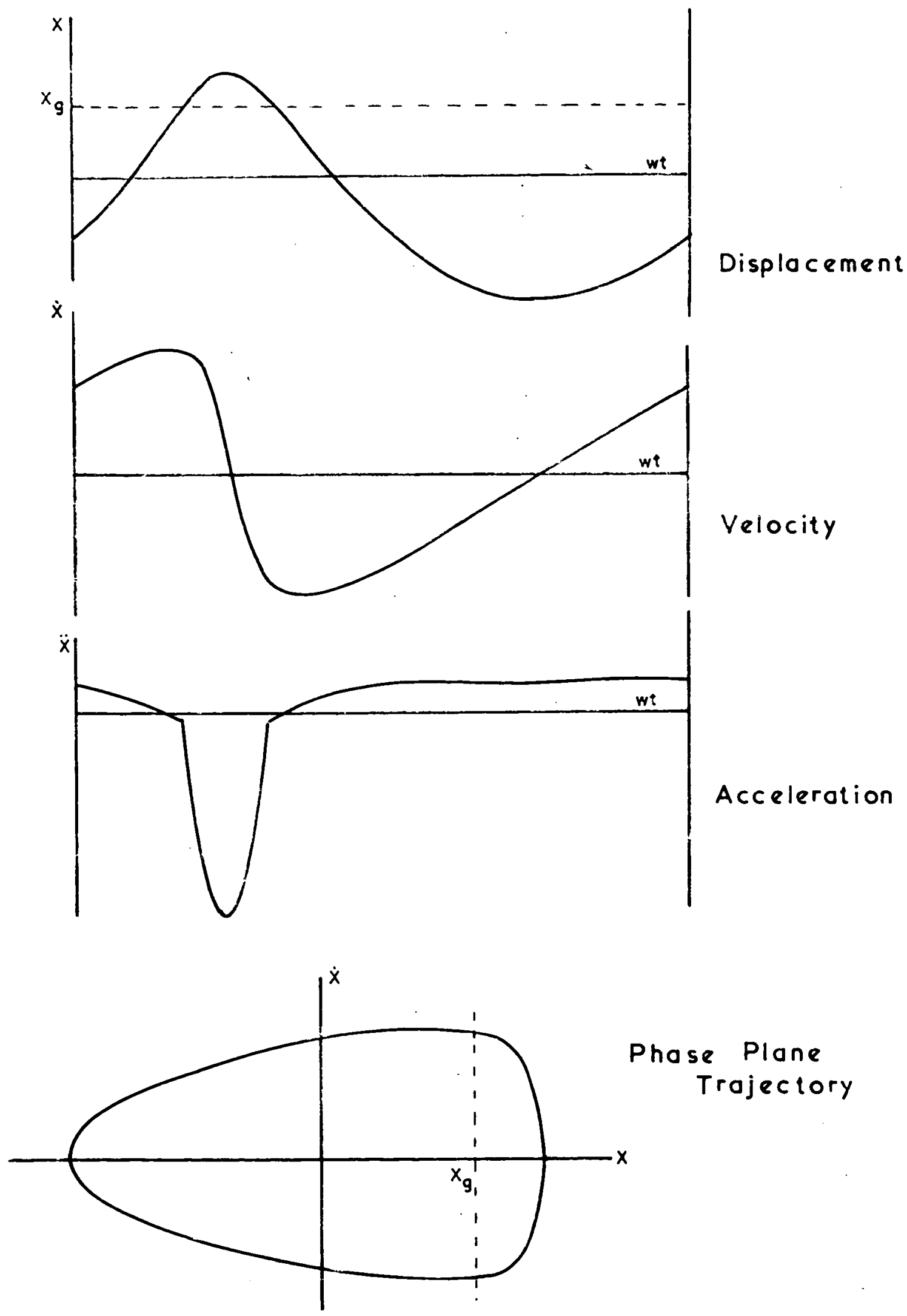

Phase Plane Trajectory

Conveyor Surfoce Motion

FIG. 2:7 
in 3.4.1. The motion may be represented by two linear equations depending on whether $X>$ or $\left\langle X_{g}\right.$, and if conditions of continuity of displacement and velocity are applied at entry to each region an exact solution may be obtained either by adjusting the initial condition at entry to a cycle or by continuing the calculation over several cycles until a steady solution is obtained. This procedure can be extended to cases where the material mass is comparable to that of the conveyor mass. The calculations involved in this would be excessive but for the use of digital computers. Their use to solve the equations is discussed in section 5 . The other convenient method of solving the equations, namely, by analogue computer, is discussed in section 4.

No calculations were attempted on the basis of any of the methods discussed in section 2.4 .2 , largely because of the calculation involved and because the degree of approximation in waveform, as opposed to the more usual amplitude, had to be good.

The nature of the solution expected is shown in Fig. 2.7. When the displacement of the conveyor $\mathrm{X}$, is less than the gap setting $\mathrm{X}_{\mathrm{g}}$, the system is linear. Once contact with the hard spring is made, a very rapid decelferation takes place and the material on the surface is 'jerked' forward relative to the corveyor.

A most convenient representation of the conveyor motion is the 'phase-plape' trajectory, also shown in Fig. 2.7. It should be noted that the term 'phase-plane' is used loosely throughout this work for want of a better description. The phase plane representation, plotting $\dot{X}$, the velocity, against the displacement $X$ only applies to autonomous systems. This method of representation when applied to non-autonomous systems allows intersections of trajectories which in the autonomous case is impossible, and which contravenes the basic requirements of a 
phase plane as it is usually defined. If the plot is drawn in three dimensions with time as the third co-ordinate, the objection is overcome. This greatly complicates the graphical procedure and tends to obscure the physical sense of the trajectory. Throughout this work the term 'phase plane trajectory' refers to a two dimensional plot of $\dot{\mathrm{X}}$ against $X$ for a non-autonomous system unless otherwise stated. 


\section{CHAPTER 3}

3. EXPER IMENTAL PROCEDURE AND DEVELOPMENT OF APPARATUS

3.1 Scope of experiments to be carried out

The experiments to be carried out could be regarded in two ways either as an investigation into the parameters and factors influencing the performance of a vibrating conveyor, or bearing in mind the difficulty, even impossibility, of finding suitable scale factors for non-linear systems, the experiments could be seen as a means of establishing a check on computer techniques by which changes in parameters could be more conveniently studied. This latter purpose is probably the more important, since while indications of how a nonlinear system with certain parameters will behave may possibly be gained from repeated experiments on a model, the exact answer can readily be found by computer techniques which would be immediately applicable to any system. Thus while many readings were required from our experimental system, it was not felt worth while to vary all parameters over complete ranges.

\subsection{Requirements of the system}

The basic experimental requirements for studying the performance of a resonance system, linear or non-linear, for the vibratory transport of materials are as follows.

(i) A force producing device - electrical or mechanical. The possible devices include out-of-balance rotating weights, electrical vibrator, or a mechanical cam or linkage. The means chosen will have regard to the nature of the forcing 
function required - whether sinusoidal or some other function, including multi-frequency excitation. The control required is that force and frequency should be separately variable and accurately known. Of the types mentioned above the electromagnetic vibrator is probably the most flexible.

(ii) A table constrained to move in one direction and forming the mass of a single degree of freedom system whose restoring spring force is linear or non-linear. If non-linear, the non-linearity should be variable. The direction of table motion should be capable of being adjusted to give horizontal and vertical components of motion. The table should havo a surface which should be smooth and should not change in any way during experiments. This surface should be capable of inclination relative to the direction of table motion.

(iii) Material to be transported. This may either be a bulk material such as sand or gravel, or a single rigid body having a surface on which it is in a state of stable equilibrium. This latter requirement is necessary if any form of analysis is to be carried out, as an arbitrarily shaped rigid body such as a single grain of sand will roll or slide depending on its configuration at the contact surface.

(iv) A means of timing the travel rate. This may be done, in the case of bulk material by establishing the mass flow rate by weighing the throughput in a given time, or for particle motion by timing with a clock electrically or manually triggered. The accuracy is obviously dependent on the travel rate and the length over which measurements are made. 


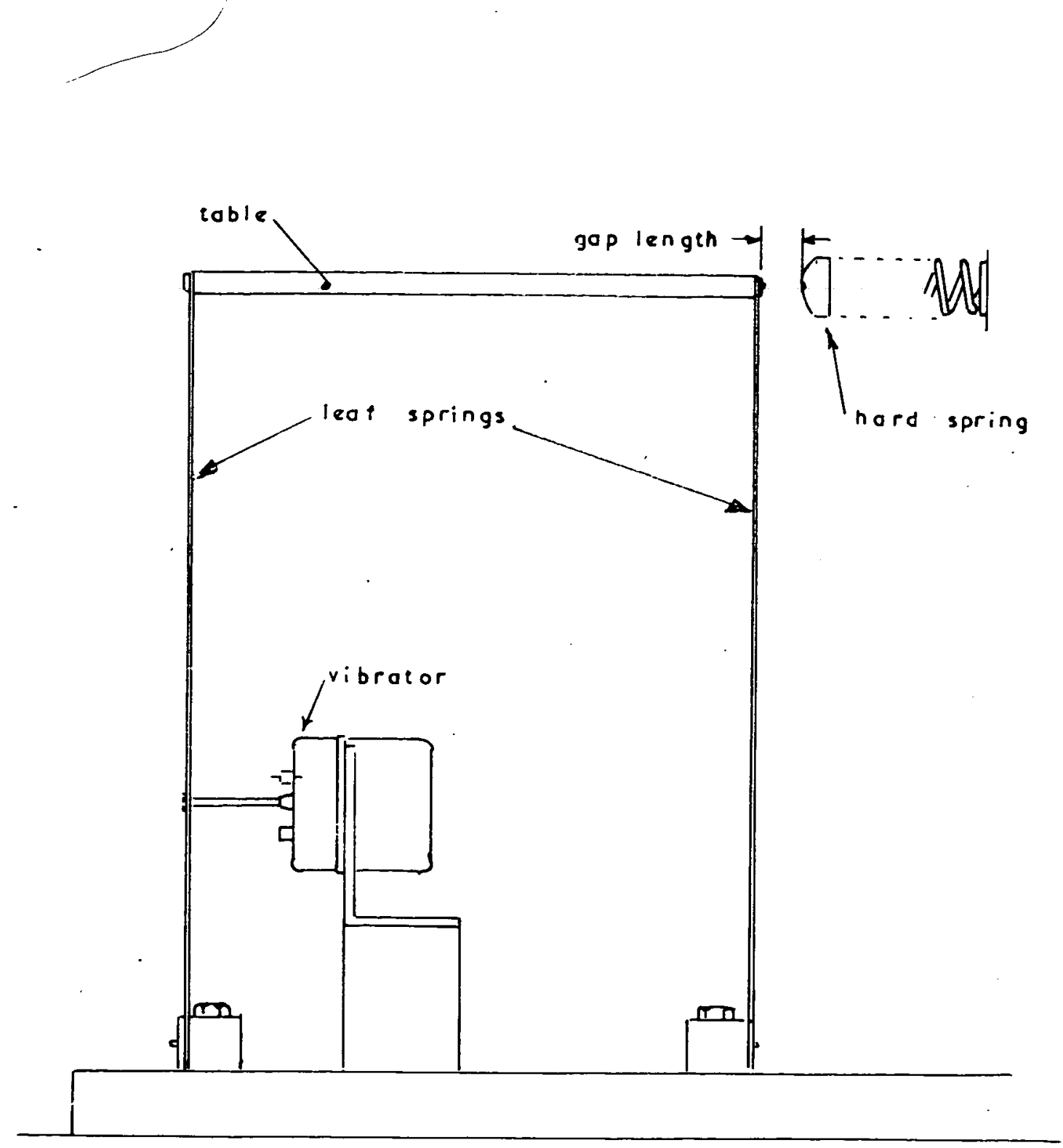

APPARATUS USED BY MACMARTIN (211))

FIG. $3: 1$ 
(v) A measure of the table displacement waveform. This is not strictly necessary but is obviously desirable.

\section{Preliminary Investigation}

The apparatus used by MacMartin (2I) is shown in Fig. 3.1. In order to allow large table amplitudes the vibrator was coupled near the bottom of the leaf springs. The non-linear spring characteristic was caused by having a stiff impact spring in the system. This apparatus was rather inflexible and did not allow for any vertical component in the table acceleration.

\subsection{Considerations affecting the design of the system}

From the reasoning above it was felt that the most suitable way of exciting the system at constant force was by means of an electromagnetic vibrator. At the time the largest available was a Goodmans vibrator model 390A. This gives a maximum force of $10 \mathrm{lb}$. approximately, without air cooling, and a maximum amplitude of $25^{\prime \prime}$ from the maker's manual, but somewhat less in practice. Since we were likely to be considering a lightly damped resonance system the amplitude restriction was more important than the maximum force. The size of the vibrator obviously had an effect on the system, so a smaller system that was probably desirable had to be considered.

The frequency range at which vibratory conveyors are normally operated had to be considered. Previous researchers quote a variety of frequencies. Berry $(2,3)$ mentions $100-240 \mathrm{r} \cdot \mathrm{p} . \mathrm{m}$. though not quoting the exact ranges his experiments covered; Booth and McCallion (4) worked in the region 400 - 1000 r.p.m; and Brickman (5) quotes 1000 - 10,000 c.p.m. as being the normal range. MacMartin 
carried out his experiments in the range $270-750 \mathrm{c}$.p.pis It was felt from consideration of these and other references that Brickman was the odd man out and that the region $5-15 \mathrm{c} / \mathrm{s}(300-900 \mathrm{c.m.p.})$ would be a reasonable frequency range to investigate. This allowed us to design our system with a resonance of the table on its linear springs in the region $8-10 \mathrm{c} / \mathrm{s}$.

In the literature of accurate amplitude and phase recording some doubt is cast on the assumption of the proportionality of vibrator force and current. To investigate this was the next stage.

\subsection{The Measurement of Force}

To quote from Prendered and Bishop (28)

"It is possible to calculate the force produced by an electro-magnetic vibrator from a knowledge of the current passing through it. Tests have been carried out however, on a small Goodman vibrator, and it was found that this method is neither accurate nor suitable for this type of work."

These authors were largely concerned with the accurate determination of phase of the forcing function as well as its amplitude and to measure force they developed a special transducer. This transducer, described in detail in their paper consisted essentially of a thin tube whose increase or decrease in length under an applied force was used to vary the distance between two lapped electrodes. These electrodes formed part of the circuit of an oscillator, and the resulting varying capacitance produced a frequency modulated signal which was amplified and demodulated. 
The previous work on vibrating non-linear systems for use as conveyors by MacMartin (21) assumed thath vibrator current was equivalent to constant vibrator force without appreciable error. This was probably due to small forces and amplitudes at the vibrator. Since in this project we were to be using a more direct coupling of the vibrator to the system and also a larger vibrator was involved it was felt that a force transducer would probably be required.

The system used by Prendered and Bishop was felt to be too complicated for our use, both in manufacturing difficulties and ancillary electronic equipment.

For the preliminary check on the linear relationship between force and current at the vibrator a very simple transducer was constructed. This consisted of a thin foil of metal with tapped end pieces for OBA connections to the system and the vibrator, the whole piece being machined from the solid. The sensing elements were wire resistance strain gauges fixed to the foil section, one on either side, so that their resistance would be increased or decreased depending on whether the force on the transducer was tension or compression. The "dummy" gauges were placed on the foil but with their axis at rightangles to the line of loading. The four gauges were combined into a resistance bridge and a transistor radio battery used to give an energising D.C. voltage. This section was obviously very prone to bending, but did work sufficiently well to justify a second transducer on a similar pattern. Further, it indicated the complete lack of correlation between vibrator current and force near a resonance of the system to which it was connected. 


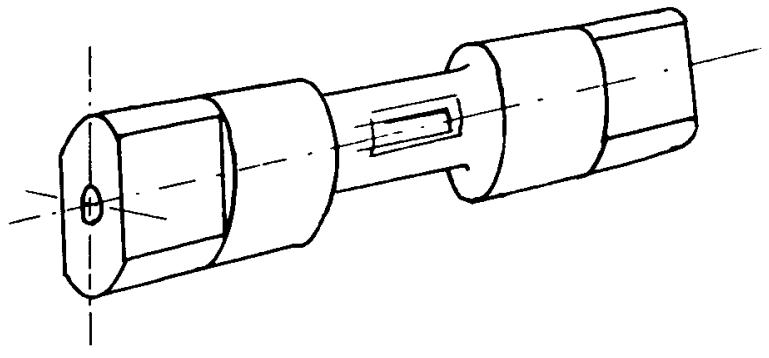

The Force Transducer

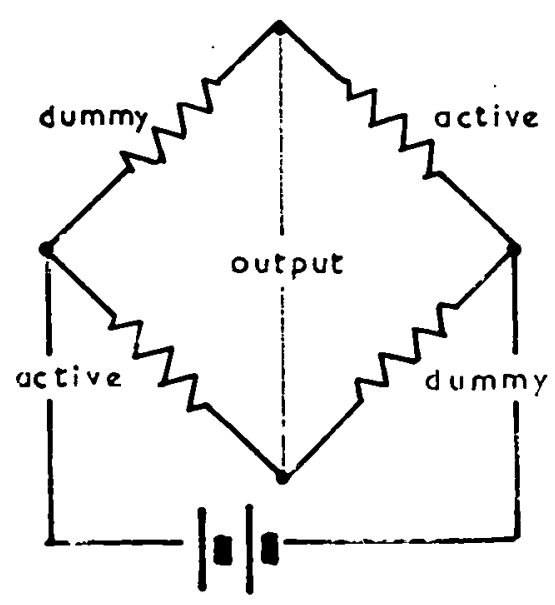

BRIDGE CIRC.UIT FOR TENSION/ COMFRESSICIN-WIRE GAUGES.

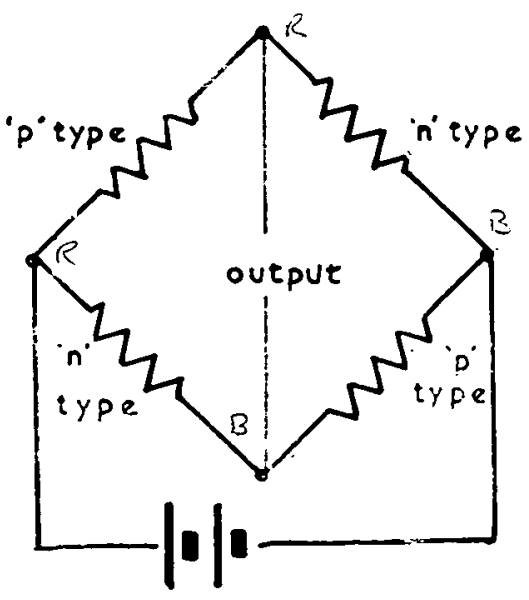

BRIDGECIRCUIT FOR TENSION/ COMPRESSION-SILICON GAUGES.

FIG. 3:2 


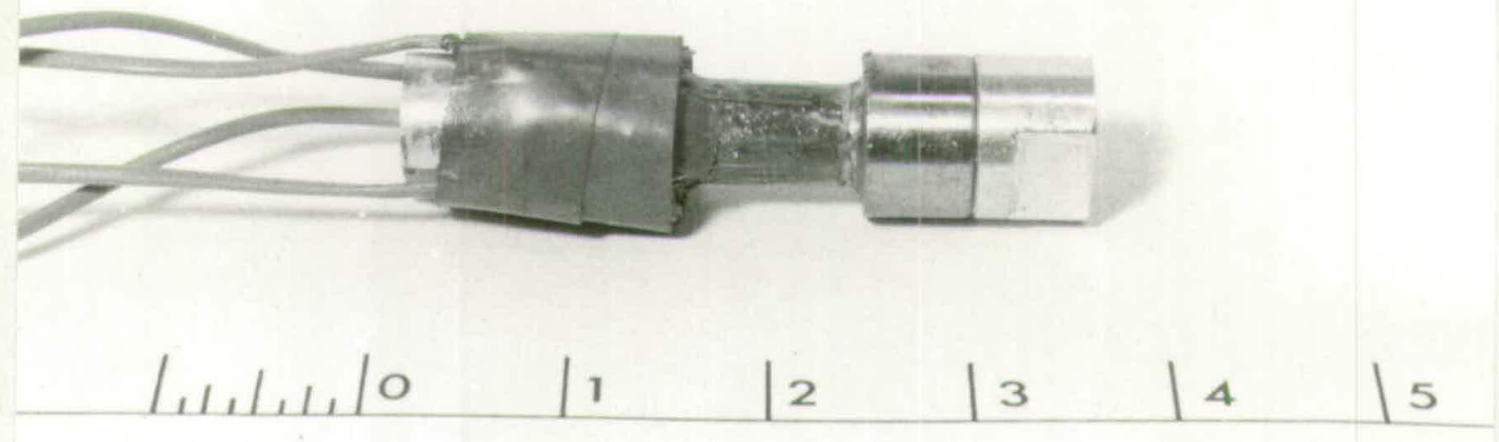

Plate 3.1 The Force Transducer with the Aluminium end pieces. The silicon gauges which are inbedded in cement for protection can be faintly discerned. 
A second more sophisticated transducer was made up and this will be described in detail, see Fig. 3.2. In this transducer, a thin tube was used having an inside diameter of ".500 and a wall thickness of about ".0065. The length of the gauge surface was about ".875 between the radii of the corners of the ends of the tube which were about "875 in diameter. These larger ends were required because whereas in the original model we could drill and tap the ends the required OBA, the inside diameter throughout the tube had to be "500 or over. The ends of the tube were thus opened out and tapped $\frac{5}{8}$ B.S.W. and alluminium plugs were made up to screw into these. The plugs were drilled and tapped OBA to provide a coupling to vibrator and the system. Aluminium was used for these plugs to keep down the weight, and this turned out to be a useful precaution as will be discussed later.

The use of the tube greatly increased the stiffness of the transducer in bending, over the original model. Despite it being extremely thin walled, no trouble was experienced due to buckling or other effects due to the short tube length.

A further sophistication was added to this transducer in that semi-conducter strain gauges were used as the sensing element instead of wire resistance gauges. These consist of thin strips of silicon cut from a single crystal mounted on a resin and glass fibre base.

With wire gauges, when measuring tension or compression only two gauges may be active while two dumny gauges must be used to make up the other arms of the bridge. Semi-conducter strain gauges of the type used have a double advantage in that not only can they be made with a gauge factor of approximately 100, but also that this gauge 
factor may be positive or negative. Thus with only tensile (or compressive) strain, both positive and negative resistive changes can be produced, and by suitable combination of gauge types e.g. by utilizing two gauges of each type, a four active-arm strain gauge bridge can be made to respond to strains of the same kind in all arms.

The semi-conducter strain gauge bridge should thus have a gauge factor of approximately 400 compared with 4 for a wire gauge bridge, all things being equal. However, all things are not equal - gauge resistance being lower ( 350 ohms against 600 ohms) and maximum permissible current being less ( 6 ma against $10 \mathrm{ma}$ ) so that the output instead of being 100 times more than that which would have been achieved with wire gauges is only of the order of 30 times better. Another disadvantage of the semi-conductor gauges was that those which were obtained for this project, being two gauges with positive gauge factors and two with negative gauge factors, were not so well matched for resistance as was the case with wire gauges, and balancing would have been extremely difficult to achieve accurately. However, the D.C. component of the output was not important, provided the dynamic output was accurate.

\subsubsection{Calibration of force transducer}

Before being used the transducer was calibrated statically and dynamically.

In the static calibration the transducer was loaded in tension or compression and the D.C. energising supply applied across the bridge. The output was fed into a Solartron Digital Voltmeter. From the graph of load against output a linear relationship was found from which a value of output in $\mathrm{mV} / \mathrm{lbf} / \mathrm{bridge}$ volt was calculated. Since in the 
experiment the transducer output was to be metered by a valve voltmeter this figure was converted into volts r.m.s.

The dynamic calibration required firstly that we calibrate an accelerometer for frequencies up to $50 \mathrm{c} / \mathrm{s}$, as without a cathode follower the response of an accelerometer is very frequency dependent at lower frequencies. The calibration was carried out by mounting the accelerometer on a spring supported block and forcing this to vibrate at various amplitudes and frequencies with a large Goodman's vibrator. The amplitude was measured by a microscope against reflected light on sandpaper.

For each frequency a linear relationship was found to exist between accelerometer output and displacement. The force transducer was then placed vertically on a large Goodman's vibrator. A known weight was then fixed to the top of the transducer, and, still in the vertical plane, an accelerometer was mounted above the weight. The procedure of exciting the system at the frequencies used for calibration of the accelerometer was repeated, and transducer output against acceleration tabulated. Again, this produced a linear relationship. As force is equal to mass times acceleration it was possible to state the force and thus find a dynamic factor transducer output in $\mathrm{mV} / \mathrm{lbf} / \mathrm{bridge}$ volt.

The transducer output factor was found to be identical for sinusoidal input to $50 \mathrm{c} / \mathrm{s}$ or to static calibration. This factor was $\quad .231 \mathrm{mV} / \mathrm{lbf} /$ volt applied

or $\quad .945 \mathrm{mV} / \mathrm{lbf}$ at the max. bridge voltage of 4.1 volts. This was a gain of approximately 30 times over that obtained using wire gauges. This gain enabled both direct display on a 


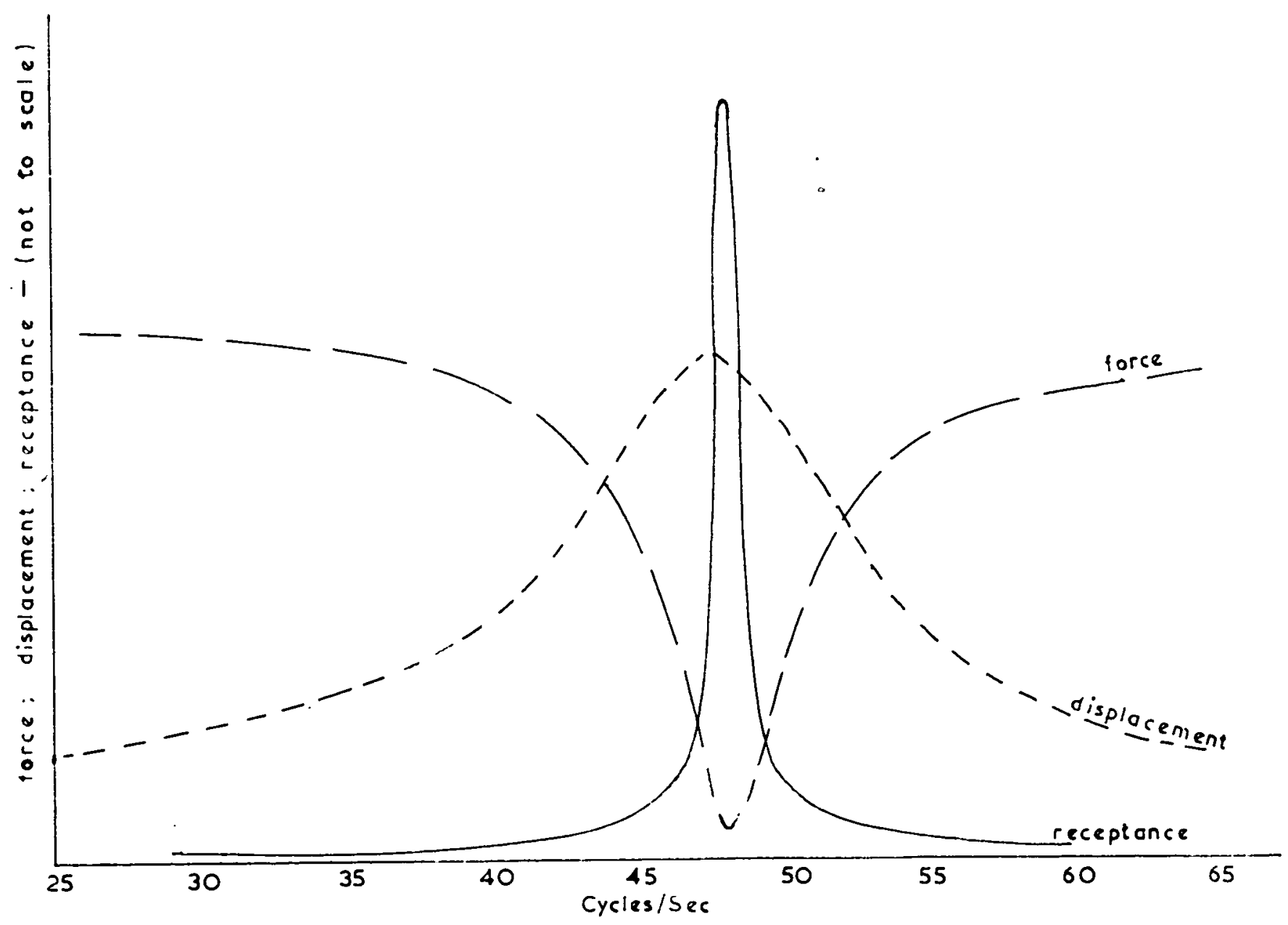

FORCE, DIS PLACEMENT, \& RECEPTANCE (DISPLACEMENT/FORCE) FOR CANTILEVER SYSTEM, VIBRATOR CURRENT HELD CONSTANT.

FIG. 3:3 
Solartron 1400 Oscilloscope with high gain amplifiers and also direct monitoring on a Phillips valve voltmeter. The noise level using screened leads was about $.07 \mathrm{mV}$ under the conditions prevailing in the laboratory. 3.5 .2 An investigation into the relationship between vibrator force

Having developed an accurate force transducer the relationship between the input current and the output force could be investigated. From another investigation there had been made up a cantilever beam, which consisted of two $I^{\prime \prime} \mathrm{x} \frac{3}{8} "$ mild steel beams held together by bolts to give an interface which introduced some friction into the structure. A wire resistance strain gauge bridge was fitted onto this cantilever near its built in end, and the vibrator connected through the force transducer to the cantilever near its free end. An anmeter was connected in the vibrator circuit so that input current could be monitored.

Then holding the vibrator input current constant the first resonance peak (at approximately $50 \mathrm{c} / \mathrm{s}$ ) was investigated. This is shown in Fig. 3.3, the displacement being measured by the output from the resistance bridge on the cantilever beam. The vibrator force was approximately $3 \mathrm{lbs}$. and the maximum amplitude at the vibrator

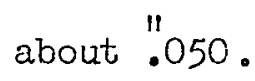

The figure shows a typical relationship for this type of system. It shows quite clearly that the force drops down to approximately zero. at the resonant frequency, and the displacement peak from the strain gauge bridge gives a response peak, which if analysed by usual bandvidith methods would give a completely erroneous measure of damping. 
This latter effect is due to the system whose response is measured not being a simple cantilever beam but a more complex system including the vibrator with its associated mechanical damping and such electrical effects as back e.m.f. If a receptance of the system is plotted, dividing the displacement by the measured force, a better estimate of the system damping is obtained, which can be checked by disconnecting the vibrator and using decrement techniques.

A further investigation was carried out into the variation in phase angle between vibraior current and force for this particular cantilever system. Force was measured by displaying vibrator current (measured from the voltage drop across a resistor in the vibrator current) and the output from the force transducer, on an oscilloscope. This was not highly accurate due to (a) the difficulty of accurate phase measurement, $5^{\circ}$ being about as good as could be guaranteed, and (b) the very small amplitudes of force at resonance. However, despite these difficulties, when the force amplitude and phase angle for constant input current were plotted on polar graph paper, as in the Kennedy and Pancu (15) technique, indications were shown of the circular curve indicating a resonance effect between force and current.

From these experiments it is obvious that near resonance, the vibrator current is, both in phase and amplitude, an inaccurate measure of the output force, and that some form of transducer is necessary. The experiments also served to establish the accuracy and reliability of the transducer for frequencies up to $50 \mathrm{c} / \mathrm{s}$ and forces up to $5 \mathrm{lb}$. 

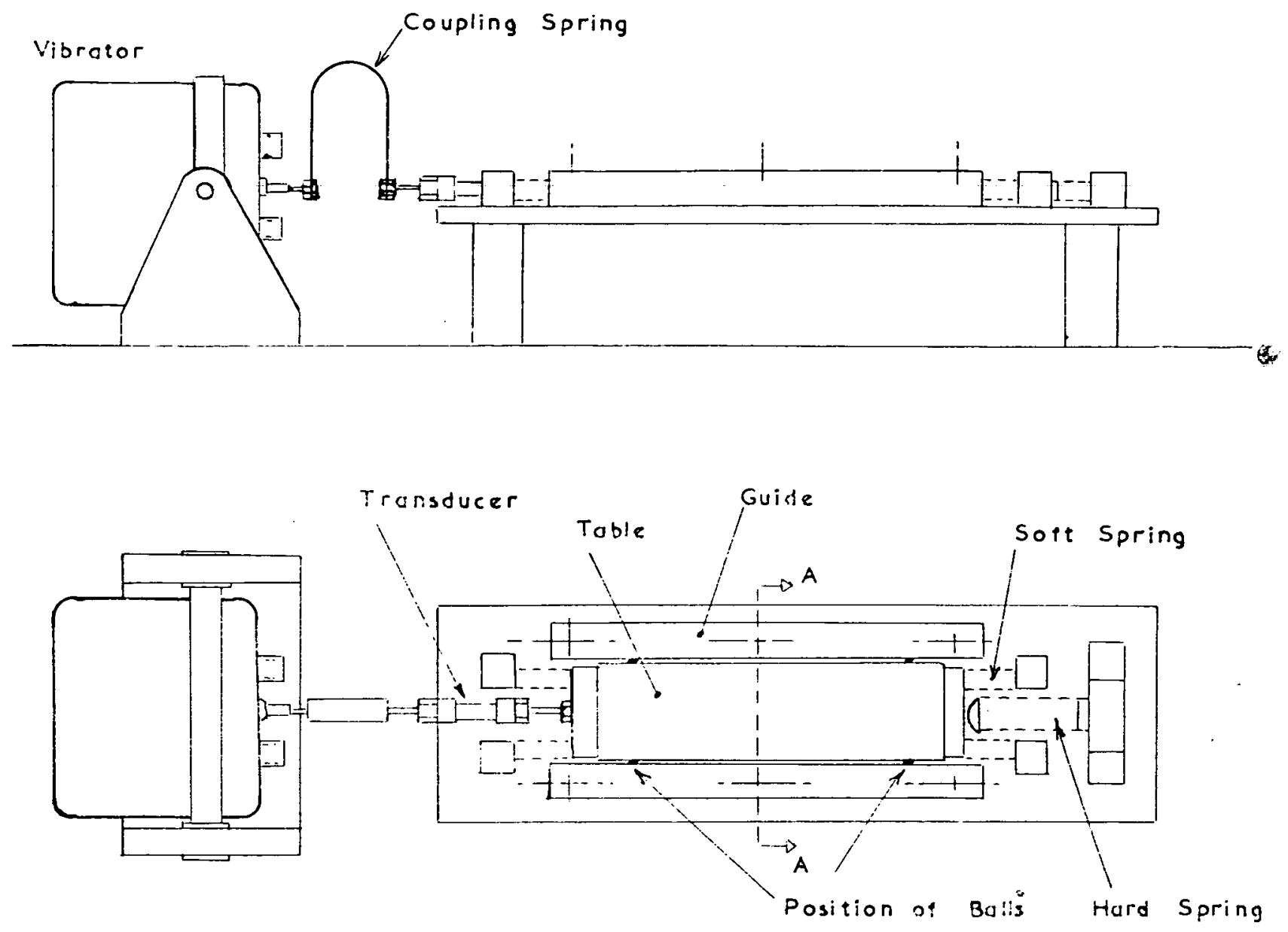

GENERAL ARRANGEMENT OF APPARATUS SYSTEM A

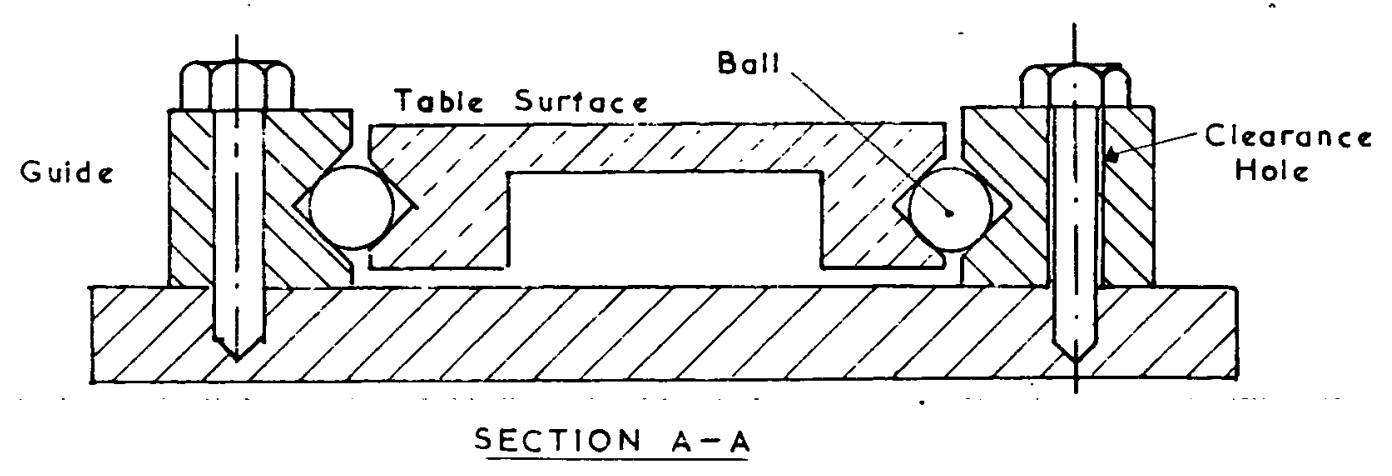

SYSTEM A

FIG.3:4 


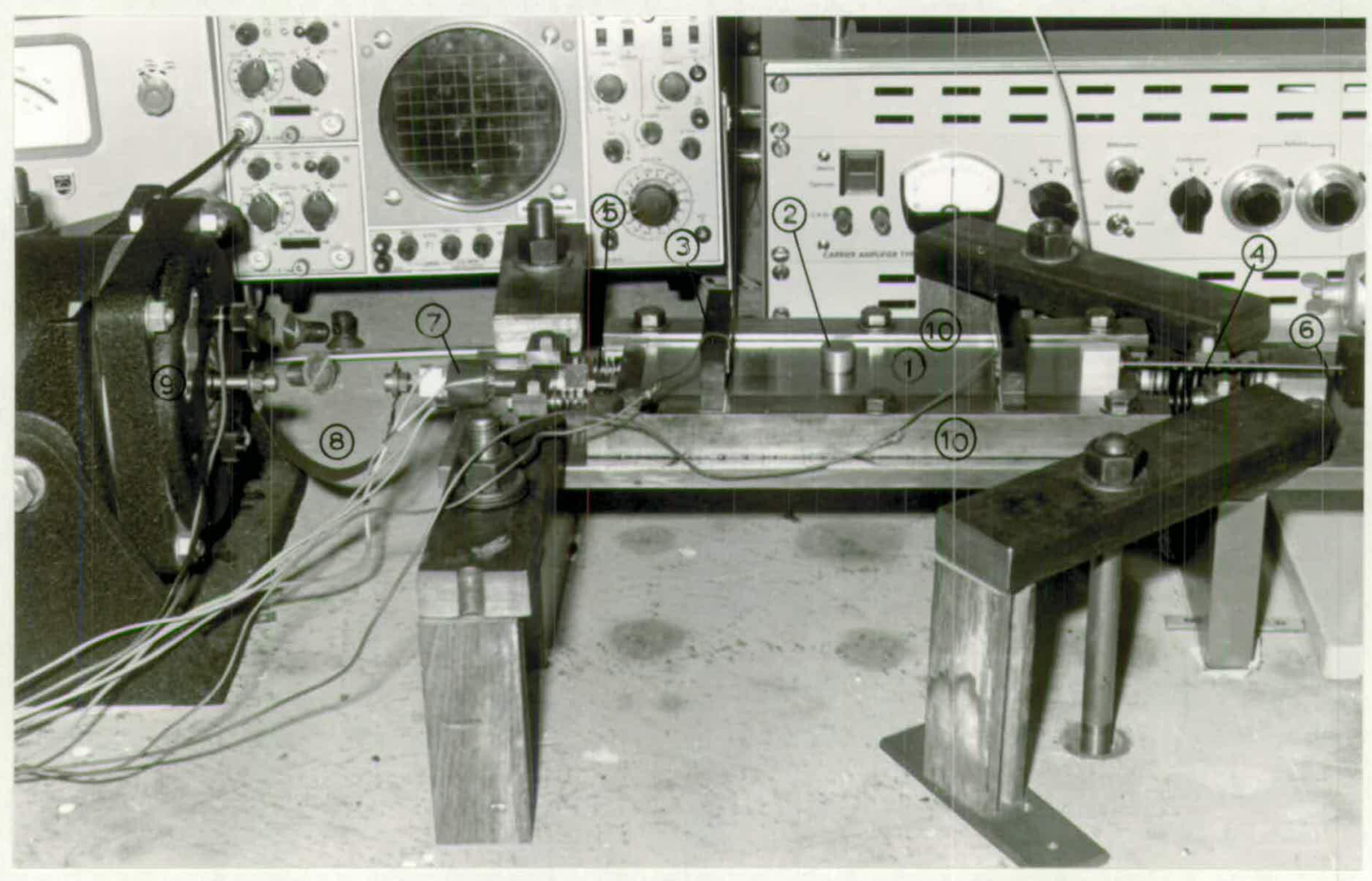

Plate 3.2 View of Apparatus System A

$\begin{array}{ll}\text { 1. Conveyor Surface } & \text { 6. Linear Displacement Transducer } \\ \text { 2. Slider } & \text { 7. Force Transducer } \\ \text { 3. Timing Contact } & \text { 8. Coupling Spring } \\ \text { 4. 'Hard Spring } & \text { 9. Vibrator } \\ \text { 5. 'Soft' Spring } & \text { 10. Guides }\end{array}$




\subsection{The Apparatus}

Two systems were used in the course of the investigation. They are referred to throughout the work as System $A$ and System $B$. System A was designed to investigate the rate of transport of material over a horizontal table whose motion was confined to the horizontal plane. System B was very similar to System A and was used to investigate in a direction inclined at an angle to the horizontal. Thus System A was for non-linear spring systems only as motion does not normally occur in this case with linear systems, while System B could be used for the investigation of linear or non-linear spring systems. 3.6.1 System A - see Plate 3.2 and Fig. 3.4.

Bearing in mind the limitations of the vibrator and the frequency range which was to be investigated a small table was made whose dimensions were $3^{\prime \prime} \times 10 "$. The guides to constrain the motion consisted of a linear ball bearing. Onto a baseplate two parallel guides were

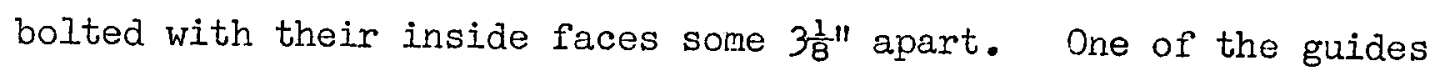
had clearance holes to allow some lateral adjustment. These guides were of I" $x I^{\prime \prime} \times 12^{\prime \prime}$ mild steel bar and had a $90^{\circ}$ vee notch in their inside faces. The table was made 311 thick and a corresponding vee notch was milled in each side of the table. When the table was fitted between the guide rails, $\frac{3}{8} "$ dia. steel balls were put into the channel formed by the notches on the guides and table, and the guide spacing adjusted to give positive location and free running. Originally it had been intended to use several balls on each side with self lubricating spacers, but experience showed that if the guides were correctly adjusted for contact pressure, the balls rolled but showed no tendency to creep down the length of the track.. 
Eventually only two balls a side were used. At first there was some slight roughness in the guides as the finish of the notches was that of a milled surface, but the balls soon removed local high spots. The horizontal surface which was to be used for the experiments was ground. The underside of the table had a channel machined out down its centre to reduce weight. Fnd pieces were attached to the table to hold the springs and transducer.

Having thus defined the vibrating mass, the required spring stiffness could be calculated. Four 'soft' coil springs were made, fitted with end pieces, and arranged symmetrically two at each end of the table.

The 'hard' coil springs were arranged with one end fitting into a mounting block which was bolted to the base plate, while the other end had a brass plug with a curved 1 " radius 'impact' surface. The mounting block has elongated holes to allow its position on the base plate to be varied and hence change the gap between the radiused surface and the end piece of the table. The gap was measured with the table in its equilibrium position.

The 'hard' spring was centrally positioned at one end of the table. In the corresponding position at the opposite end the force transducer was connected by an OBA rod.

It had originally been planned to couple the vibrator directly to the force transducer, and hold the transducer output constant by varying the input vibrator current, while exciting the system at various frequencies. However it was soon found that this method would not give the required effect. Not only did the damping of the vibrator damp out interesting phenomena and its amplitude restriction give 
trouble, but the mass of the moving parts in the vibrator caused an inertia force to be transmitted to the transducer during the period of rapid deceleration following the contacting of the hard spring. Thus the transducer output was of a sinusoidal force plus a superposed non-sinusoidal inertia force. To obviate these problems a soft spring was coupled between the force transducer and the vibrator. This spring was in the form of a ' $U$ ' shaped piece of spring steel strip. This proved successful in acting as a mechanical filter and preventing the inertia force of the moving mass of the vibrator from reaching the transducer. It also effectively uncoupled the damping of the vibrator from the table..$^{I}$ By making the system a two degree of freedom system it allowed amplification of table motion without causing over-travel in the vibrator at some frequencies, but aggravated it at others. There was, thus, a limitation on the neximum force which could be used over the frequency range of approximately $\mathrm{l} \mathrm{lb}$. Because of its shape the soft spring had a tendency to cause a bending moment to be transmitted to the force trasducer.

The proof of the separation of the vibrator and table systems can be seen in the linear equations, where $\mathrm{m}, \mathrm{k}, x$, are vibrator mass spring and damping coefficients, $k_{1}$ the coupling spring stiffness, and $M, K$ and $\gamma_{2}$ the mass spring force and damping of the table system, $z$ is vibrator amplitude; $X$ is table displacement.

$$
\begin{aligned}
& m " \prime \gamma_{1} x+k_{1}(x-X)+k_{2} x=F \sin \omega t \\
& M " x+\gamma_{2} \dot{X}+K(x)+k_{1}(x-x)=0
\end{aligned}
$$

$\vec{F}$ is not held constant. The force which the transducer measures is the force in the soft spring, $k_{I}(x-x)$. While $x$ is an almost pure sinusoidal motion, it cannot be argued that $X$ is. However in cases where the waveform was extremely non-linear, i.e. at low frequencies, $\mathrm{X}$ was large in comparison with $X$. At higher frequencies alternating component of $X$ was more nearly sinusoidal. On an oscilloscope no divergence of the transducer output from sinusoidal could be noticed. 


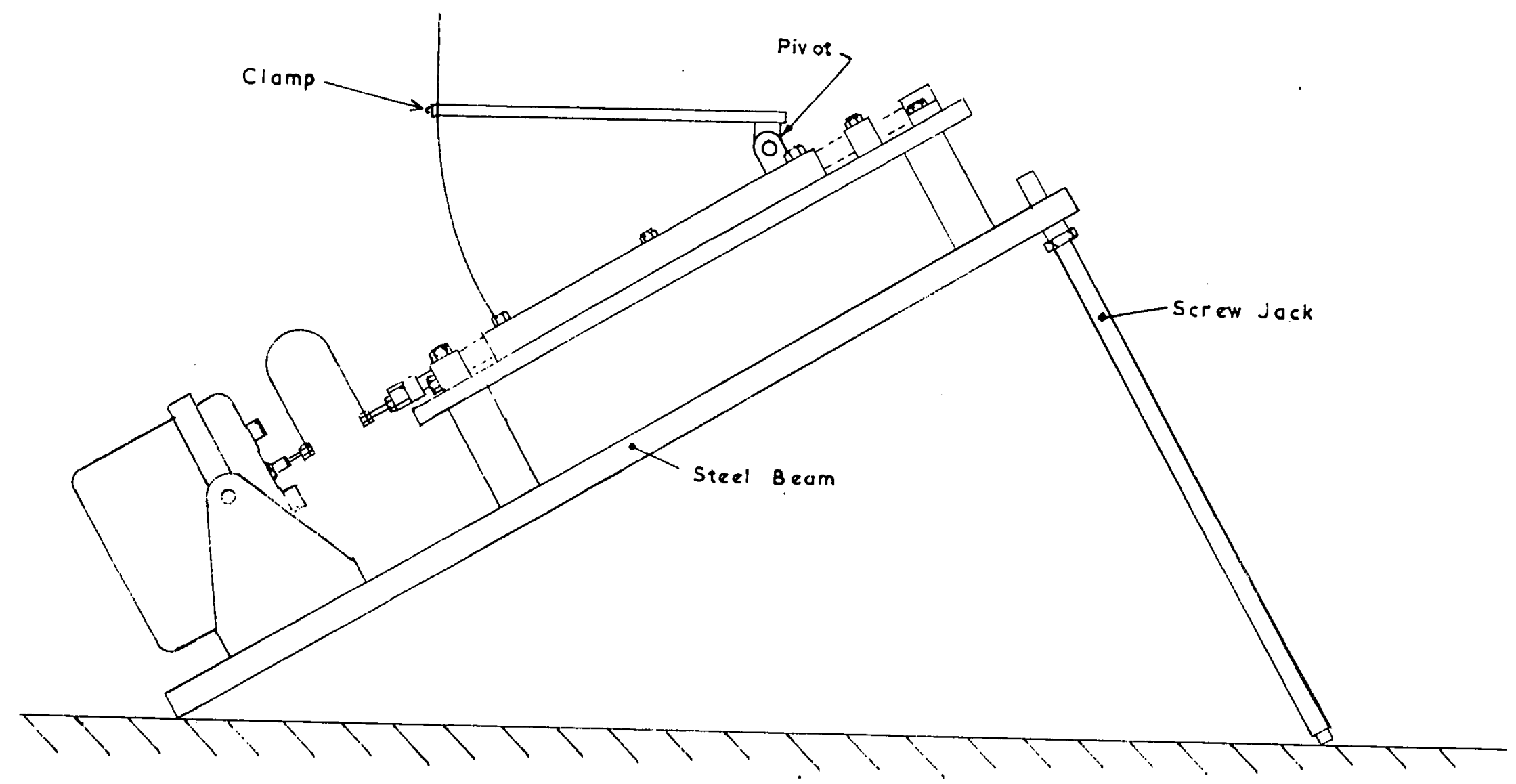

General Arrangement - SYSTEM B

FIG. $3: 5$ 


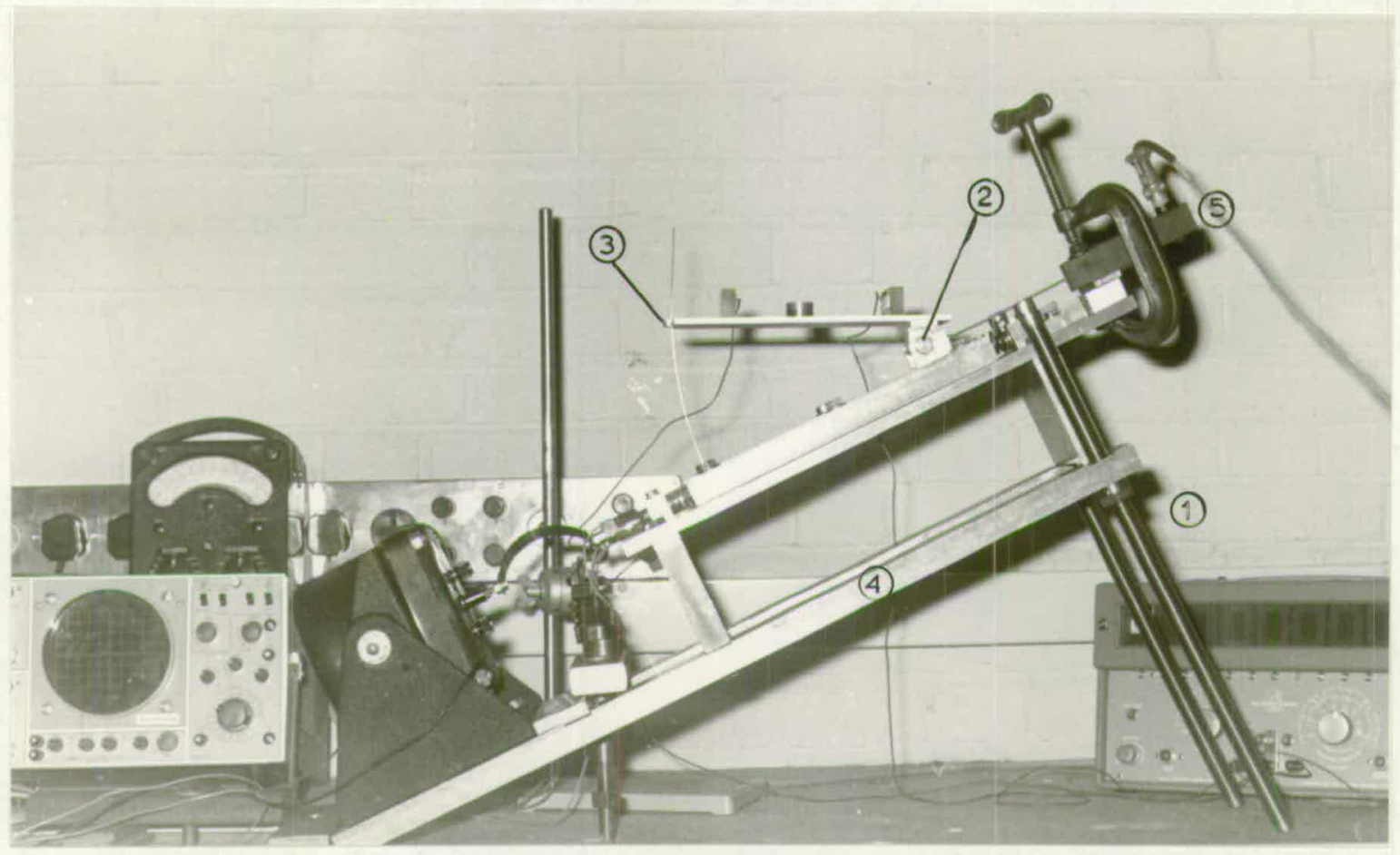

Plate 3.3 View of Apparatus System B
1. Screw Jack
4. Base Plate
2. Pivot
5. Linear Displacement Transducer
3. Clamp 
This was reduced by using cone washers on all connections to the spring to make its end conditions more those of a pinned end than a built-in end condition. The assumption that any bending noment transmitted did not affect the accuracy of the force transducer was corroborated by the agreement between displacement amplitudes of the linear system from experiment and the equivalent system as set up on an analogue computer see graph $3: 1$.

3.6.2 System B - see plate 3.3 and Fig. 3.5

System $B$ was a modified version of System $A$, the changes being immediately obvious by referring to the plate 3.3 or Fig. 3.5. Two steel beams $2 " x$ l" were used to support the entire vibrator-baseplate and system, forming a rigid location between vibrator and table which did not exist in System A. At the end of these beams away from the vibrator holes were drilled and threaded with a $\frac{3}{4}$ B.S.W. Thread. Then by screwing long $\frac{3.11}{4}$ B.S.W. screws down through these holes onto the concrete plinth on which the apparatus stood, the entire system could be jacked up to any angle of inclination up to $30^{\circ}$. In order to maintain a horizontal conveying surface at any angle of inclination a new table was designed. The suspension, spring mounting and force connection remained as before. The difference was that the table was replaced by a carriage of approximately the same dimensions as the table in System A. However, instead of the conveyor surface being part of the carriage, a surface was arranged above it on a pivot which enabled it to be tilted in any angle up to $30^{\circ}$ and clamped there, so that it could always be maintained horizontal regardless of the angle of inclination of the rest of the apparatus. A clamp which gripped a steel strip helped to lock the table surface in position. As before 


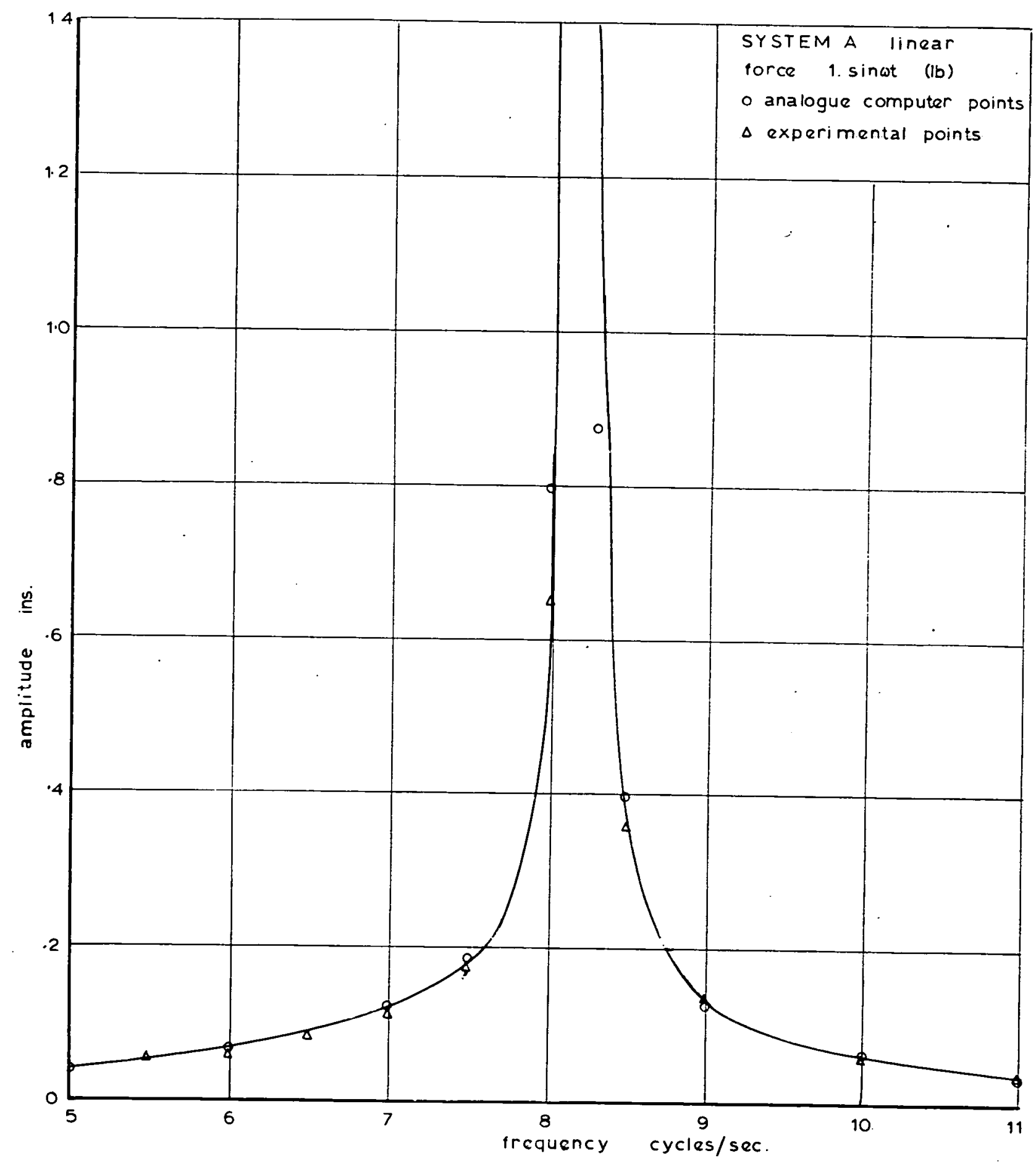

RESPONSE OF SYSTEM A-EXPERIMENTAL \& ANALOGUE COMPUTER RESULTS. 


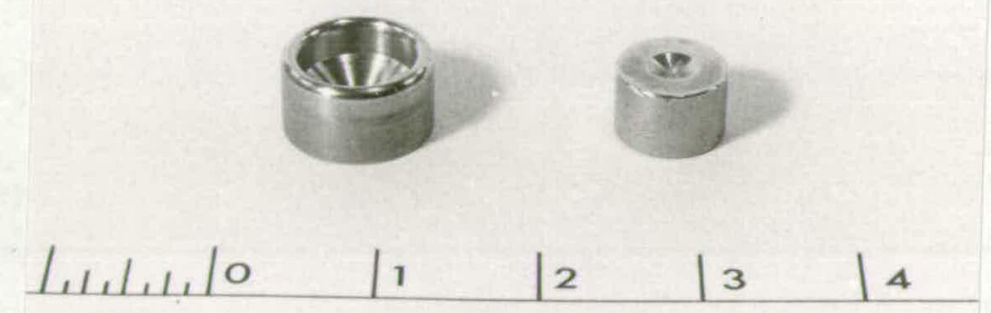

Plate 3.4 Sliders - Turned upside down to show sliding surface. The smaller slider (right) is slightly recessed. The larger (left) is almost hollowed out'. 
the table surface was of ground steel. This new system weighed somewhat more than the original one, and to keep the natural frequency of the linear system in the region $8-10 \mathrm{c} / \mathrm{s}$ new 'soft' springs were made up.

The constants of damping, spring stiffnesses etc., for System A and System B will be given in Appendiz $A$.

3.6 .3

The material to be transported was chosen to be a rigid body for the bulk of the tests. The 'sliders' can be seen in plate 3.4 They were made from round bar to avoid any problem in timing the travel rate due to slider rotation. Brass was shown to be a convenient material to use, particularly as it was unlikely to damage the table surface. The smaller of the sliders was of $\frac{3}{4}$ " diameter brass bar. Its face was as smooth and flat as could be achieved using the lathe and einery paper. As high spots at the centre tended to cause spinning the centre was slightly recessed. All edges were slightly rounded. This slider proved fairly satisfactory, but for reasons which will be discussed later a second slider vas made. This was slightly larger, being I" dianeter. Its centre was completely recessed so that the only bearing surface was round the outer edge. This lip was turned to give a radiused surface so that the only contact was round the circumference of a circle. This slider proved wholly satisfactory. Some experiments were carried out using sand as the material to be transported and these are described in Appendix C . 3.5 .4

The timing equipment was that used by the author in a project carried out some years previously. This was an electrical 
'make-and-make' contact system using the automatic gating facility for interval timing on a Venner digital time and frequency meter. Two 'Tufnol' bridge pieces were fitted onto the table surface a set distance apart. On the face of each was a brass strip which protruded from the bridge piece with a lead to the frequency meter, the table surface being an 'earth'. On contacting the first strip the brass slider caused a pulse which automatically started the meter, and the pulse which it caused on contacting the other strip stopped the meter. This system of course depended on the slider being of some electrically conductive material. The possible accuracy in measuring the time interval was far beyond the accuracy of the experiment, but the automatic nature of the timing removed some human error. 3.5 .5

Two methods were used to measure table displacement. One was the smoked glass method whereby a pointer on the table scratches a trace on a smoked glass plate drawn over it in a direction orthogonal to the table motion. While this method is slow and messy, it is fairly accurate particularly for larger amplitudes. The other method attempted was to use a Honeywell Linear Displacement Transducer. In this type of transducer the table motion is used to move an alloy billet inside two coils. The variation in inductance between the coils is used to give a modulated signal proportional to displacement which can be amplified and denodulated. This method was not very satisfactory because of non-linearity in the response of the transducer but it did prove useful in identifying the nature of the table motion even if unsuitable for amplitude measurements. 


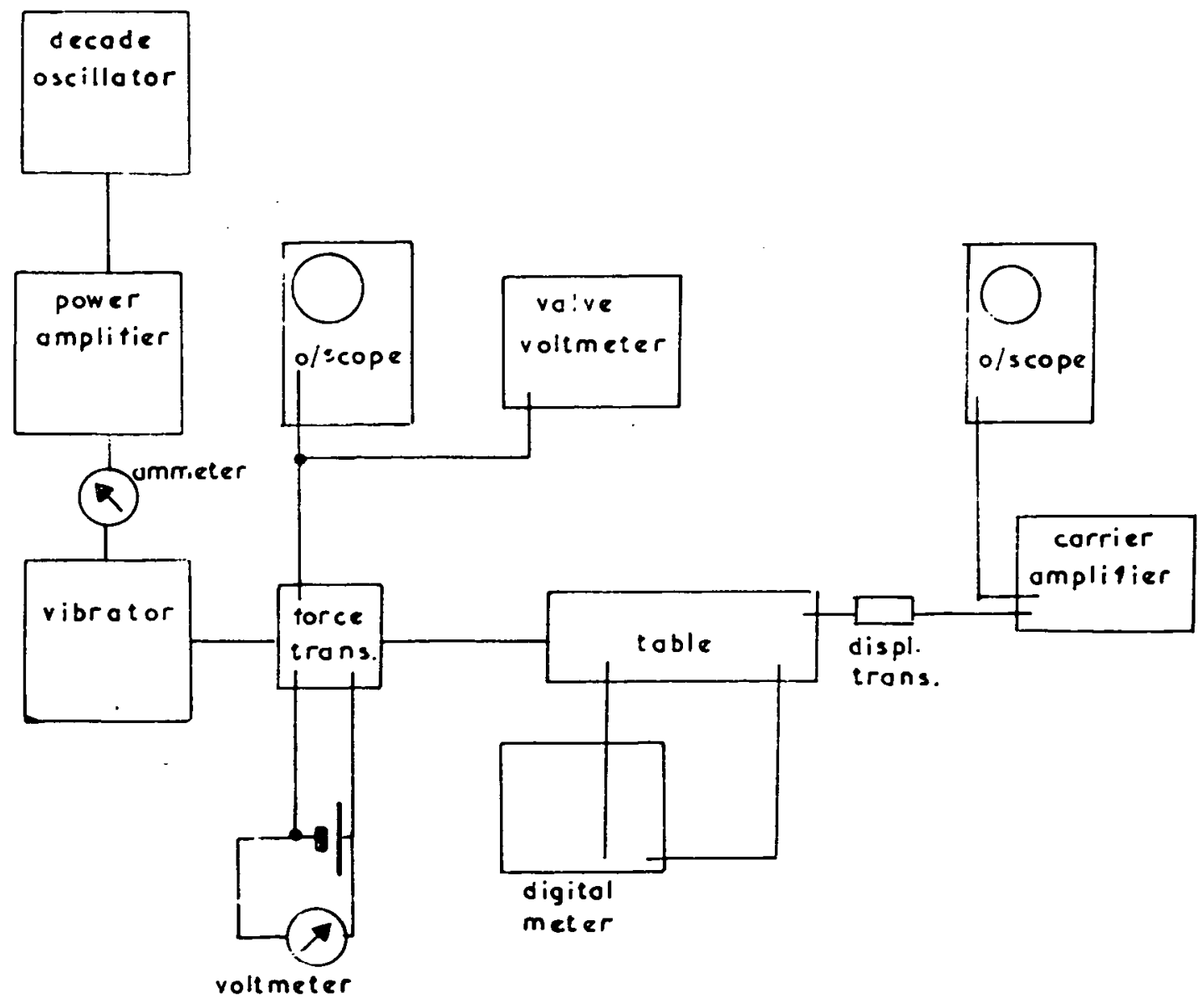

BLOCK DIAGRAM OF ANCILLARY EQUIPMENT

F:G. $3: 6$ 


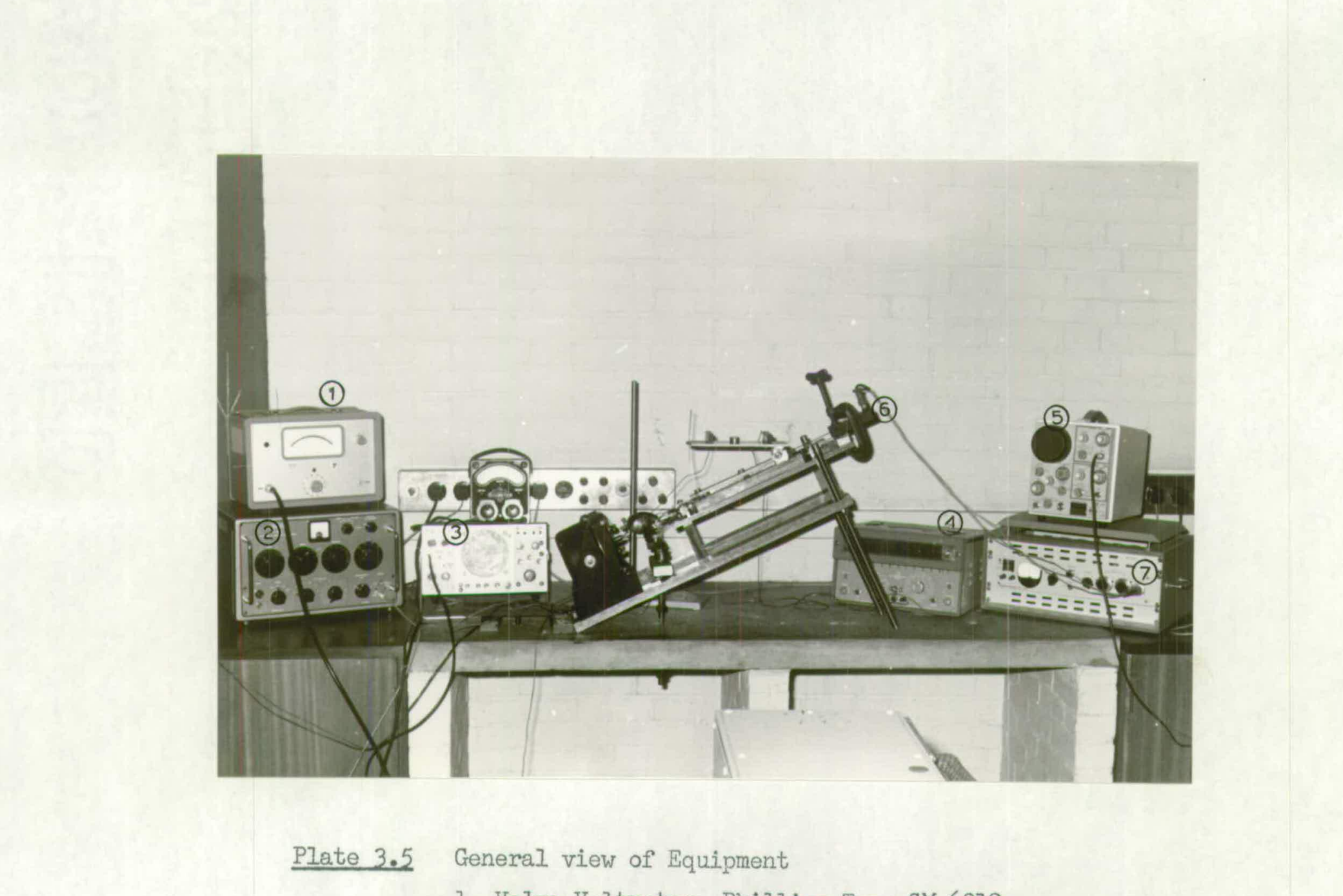




\subsubsection{Ancillary Equioment Fig. 3.6}

The vibrator was excited by means of a Muirhead Low Frequency decade oscillator type D-880-A, the signal being amplified by a Goodmans power amplifier type PA50 VA. An ammeter was connected in the vibrator circuit. This system gave the required range of force, with a frequency control of .I cycles/sec. The oscillator frequency control was checked by a digital frequency meter and it was found that the dial settings were completely accurate. The energising D.C. supply for the strain gauge bridge of the force transducer was from a PP9 transistor radio battery with a resistance to reduce current to the maximum permissible value for the gauges. A voltmeter was used to monitor the supply voltage. The output from the transducer was displayed on a Solartron CD 1400 with high gain amplifiers and also was fed in to a Phillips GM6012 valve voltmeter. As previously described the leads from the timing contacts were fed directly into a Venner 3336 digital time and frequency moter. The unsuccessful Honeywell displacement systein was a linear displacement transducer type ID 12 whose maximum amplitude was I $\frac{1}{2}$ together with a Carrier Amplifier type 2506 which contained an oscillator, amplifier and demodulator. The output from this could either be displayed on a meter or shown on an oscilloscope.

\subsection{Experimental Procedure}

The constants of the two systens used were found as described in Appendix A where their values are tabulated.

Before taking a reading the non-linearity had to be set up in the springs. This was done by using one of three hard springs and adjusting the gap setting between the contact surface of the hard spring and the table in its equilibrium position. Feeler gauges 
were used for this, an considerable care had to be taken to ensure that the gauge was not itself deflecting the table. The equilibrium position of the table presented some difficulties as there was occasionally a feeling that the vibrator produced a small D.C. component. In an attempt to check this the output from the force transducer was displayed on the CD1400 oscilloscope using the D.C. amplifiers so that any static force would show. A further precaution was that the gap was set with the power amplifier connected to the vibrator. Because of the high gain of the power amplifier there was some signal output from it even when the oscillator was at its highest attenuation. However, if the frequency of the oscillator signal was increased to above $50 \mathrm{cycles} / \mathrm{sec}$. no response of the system was caused by the small force that came through the low pass filter of the spring. It was hoped that in this condition any D.C. component would be present and compensated for in the gap setting. When using System $B$ the angle of inclination of the guides obviously had to be set before the gap as the table equilibrium position was varied by the addition of an $\mathrm{mg}$ sing gravity term. The angle of inclination of the guides was set using an adjustable square with a built-in level and vernier.

To protect the table surface from $r$ sting it was kept under an oil film when not in use. Before readings could be taken it was degreased using trichloroethylene and polished using Brasso metal polish. In theory it would have been desirable to measure the coefficient of friction between the slider and table surface each time the table was polished, but this would have required some dismantling of the apparatus. To avoid this a given configuration was adopted as a yardstick and this was used to check the conveyor performance by comparing the travel rate 
with that previously obtained. As travel rate is dependent on friction this was a fairly rigorous test. Despite all reasonable precautions the maintenance of constant coefficient of friction presented real problems which became so acute under humid climatic conditions as to necessitate temporary suspension of experiments.

The 'Tufnol' pieces with the timing contacts were stuck onto the table surface using a double sided 'Scotch' tape. This gave a very rigid clamp that had the advantage of easy removal and also that it did not damage the surface. The distance between the contacts was usually 5 - 6 inches, and was accurately measured each time the contacts were set up.

Having set up the system the travel rate of the slider across the table surface was investigated over the frequency range which covered the resonance peak of the particular mass/spring system, input force being held constant usually at a value of .75 lb. The system was set to vibrate at the particular frequency and the attenuation of the oscillator adjusted to give the required output from the force transducer. The slider was then put on the plane and gently drawn back against the direction of travel until it touched the first contact. The counter was started by the contact and the time measured until the slider had traversed the table surface and made contact with the other brass strip. Great care had to be taken to ensure accurate starting, neither holding the slider against the first contact or bouncing it off the contact. This proved to be extremely difficult in those cases which involved large amplitudes of table motion and good travel rates. In attempts to overcome this many readings were taken for each point and an average value taken. In most cases it was possible to hold the 
scatter to $\pm 5 \%$ of the mean value. However with the fastest transit times of about 2 seconds this was not altrays possible. Part of the cause of this difficulty could be attributed to a 'directional preference' in the smaller of the two sliders. This effect manifested itself in that there was a certain attitude of slider alignment in which its transport rate was greatest. This was checked by inscribing a line on its top surface across a diameter and timing the travel rate for various angles of this line to table motion. Most of the results from System A were taken using this slider, the fastest angular attitude being used. This effect was most marked at the larger amplitude cases whose travel rates are thus open to doubt. A fuller discussion of the phenomena is given in Appendix D. It was not found when using the larger slider.

Because of the complicated regions of motion of the table some indication of table displacement was required. In early experiments smoked glass traces were used, and from them could be found the nature of the table motion, whether harmonic or subharmonic, and the number of times the table contracted the hard spring as well as a measure of the amplitude. For the later experiments the displacement transducer was used. This gave a convenient display of the form of the table motion, but not an accurate indication of amplitude. A rather more basic approach to the problem was to listen to the distinctive tapping of the table against the hard spring. It soon became possible to identify the type of motion in this way. Another more accurate indication could be obtained by examining the output from the force transducer as displayed on the oscilloscope. At the instant of contact the 'impact' inertia force of the aluminium end piece caused the transducer 
tube to give a small high frequency pulse at its natural frequency. On the oscilloscope trace this showed as a brief blur, thus enabling the number of impacts to be counted. It was not assumed that this small signal affected the value voltmeter output.

The brass sliders described above were the only rigid bodies used. Thus only one value of the coeffici. nt of friction was investigated. The force was sinusoidal with an amplitude (peak) of .75 Ib. unless otherwise stated. For system A three hard springs and three gap settings, making nine configurations in all, were studiex. In System B the same nine configurations together with the linear case were investigated for angles of inclination of the guides from $0^{\circ}$ to $30^{\circ}$ by $5^{\circ}$ intervals. The readings were usually at $\frac{1}{2} \mathrm{cycle} / \mathrm{sec}$. intervals of frequency, but closer where more complex regions were involved. Some experiments were carried out using $f$ : 1o sind as a bulk material. These are described in Appendix C In the cases where experiments at an angle of inclination were covered, the regions in which throwing occurred were not considered. The results are shown graphically in Chapter 6 . 3.8

The performance of the apparatus proved at all times to be most satisfactory with the exception of the displacement transducas. The efficiency of the table bearing arrangement can be estimated from the fact that with a slightly loose adjustment allowing a play of about .001 the damping of the system was .012 of critical damping. The bearings showed no real signs of wear until the experiments with sand when grit in the bearings caused some damage. The force transducer behaved very well the only trouble being caused by the fragility of 
the connections within the strain gauges which neccessitated the replacement of two gauges.

The small scale of the table model imposed by the non-availability of large amplitudes electromagnetic vibrators prevented extension to the case of a slider whose mass is greater or equal to that of the table, as there was no means of obtaining a weight of 5 lb., with its centre of gravity fairly low, within the limits of the system. These cases were investigated by analogue and digital techniques.

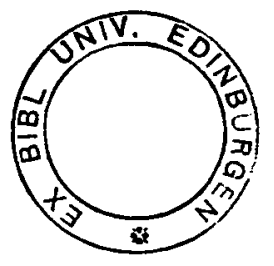




\section{CHAPTER 4}

4. AN APPROACH TO THE PROBIEM USING AN ELECTRONIC ANALOGUE COMPUTER

4.1 Introduction

The electronic analogue computer is a model in which the physical variables of a real system are represented by voltage variations in electric circuits. The independent variable of the somputer is time, so it is particularly suited to applications in dynamics where accelerations, velocities, displacements and time-varying forces are involved, both transient and steady state solutions being readily obtained.

The computers available in the department were a small Solartron 'Minispace', a Solartron model SC 30 and a Solartron type 247. The relative capacities of these machines may be assessed from the number of amplifiers. These are, for the Minispace' 10 summer/integrator groups; for the SC 30 - 10 summers and 20 summer/integrator groups; and for the $247-8$ summer and 16 summer/integrator groups. The eight summer groups of the 247 are conveniently used with additional 'plug-in' circuit cards to give limiters, relays etc. The SC 30 and 247 models could be used separately or coupled together and run as a single machine.

A full account will be given of a simple investigation using the small 'Minispace' machine, before developing the more complex circuits used later.

\subsection{The 'Minispace' analogue computer}

This computer was used for early work due to its simplicity of operation and greater availability compared with the other machines. 
Having only 10 operational amplifiers the capacity of the machine is not large. It was, however, used successfully for the study of the single degree of freedom system which represented the table vibrating in the horizontal plane with a slider whose mass was negligible compared with that of the table.

The 'Minispace' has ten operational summer/integrator groups. Each group when used as a summer offers a gain of unity on three inputs, and ten on two inputs. Five of the amplifiers when used as integrators offer the same gains as the summers, the other five having a further gain of ten times on all inputs. Coefficients of input and output quantities are adjusted by means of linear potentiometers, one end of which is permanently earthed. In addition to twenty such potentiometers, two associated with each amplifier, are four ' free' potentiometers used in conjunction with four pairs of diodes which allow the generation of discontinuous functions. The machine generates a 100 volt reference signal, coefficients being set by balancing a potentiometer against a direct reading supplied from the reference source. Potentiometer settings and amplifier outputs could be read off from a digital voltmenter. Other facilities of the machine such as a servo-multiplier were not used.

Additional equipinent used with the computer incluaded a luw frequency sine wave generator, a Kelvin and Hughes single channel pen recorder and an oscilloscope.

\subsection{The basic computer circuit for solving the single degree of freedom}

The principles of analogue computing are too well known to merit much discussion, and many references are available.

Figs. $4.1-4.2$ show the basic computer symbols, and the circuit 

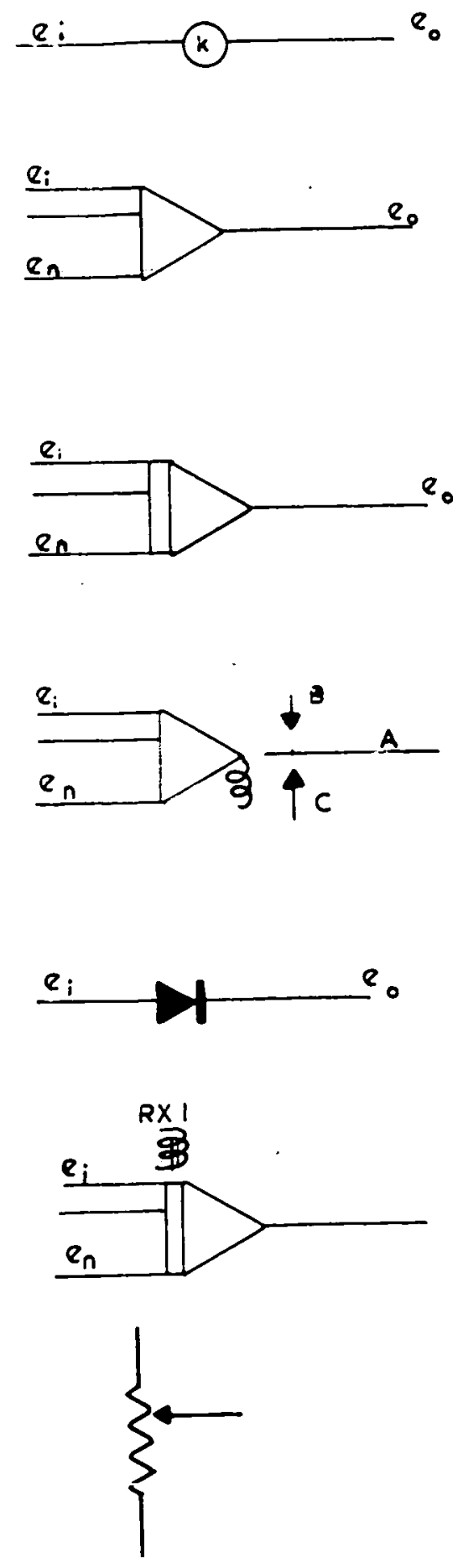

LINEAR POTENTIOMETER $e_{0}=k e_{i}$

$$
\begin{aligned}
& \text { SUMMER } \\
& e_{0}=-\left(e_{i}+\ldots+e_{n}\right)
\end{aligned}
$$

$$
\begin{aligned}
& \text { INTEGRATOR } \\
& e=-\int_{0}^{t}\left(e_{i}+\ldots+e_{n}\right) d t
\end{aligned}
$$

\section{RELAY AMPLIFIER}

$A$ is connected to $B$ if
$-\left(e, t \ldots+e_{n}\right) \geqslant 0$

$A$ is connected to $\mathrm{C}$ if

$-\left(e_{i}+\ldots+e_{1}\right)<0$

$$
\begin{aligned}
& \text { DIODE } & & \\
e_{0} & =e_{1} & \text { if } & e_{1}>0 \\
& =0 & & e_{i}<0
\end{aligned}
$$

$$
\begin{aligned}
& \text { INTEGRATOR ON } \\
& \text { RELAY CONTROL }
\end{aligned}
$$

FREE POTENTIOMETER

basic anAlogue SUB - CIRCUIT SYMBOLS

FIG. 4:1 

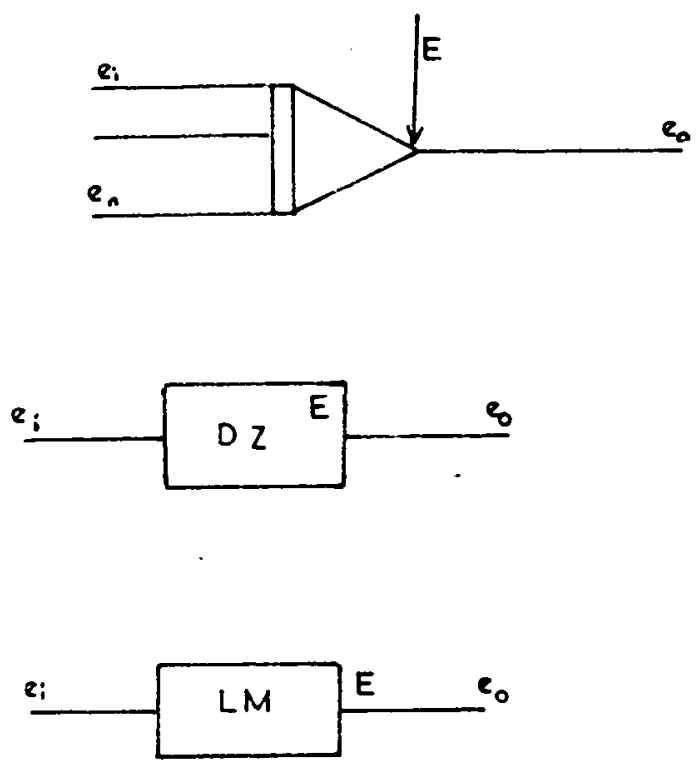

INTEG RATOR WITH INITIAL CONDITION

$e_{0}=-E-\int_{0}^{t}\left(e_{i}+\ldots+e_{n}\right) d t$
LIMI TER

$e_{0}=E \operatorname{sign}\left(e_{i}\right)$

BASIC ANALOGUE SUB - CIRCUIT SYMBOLS

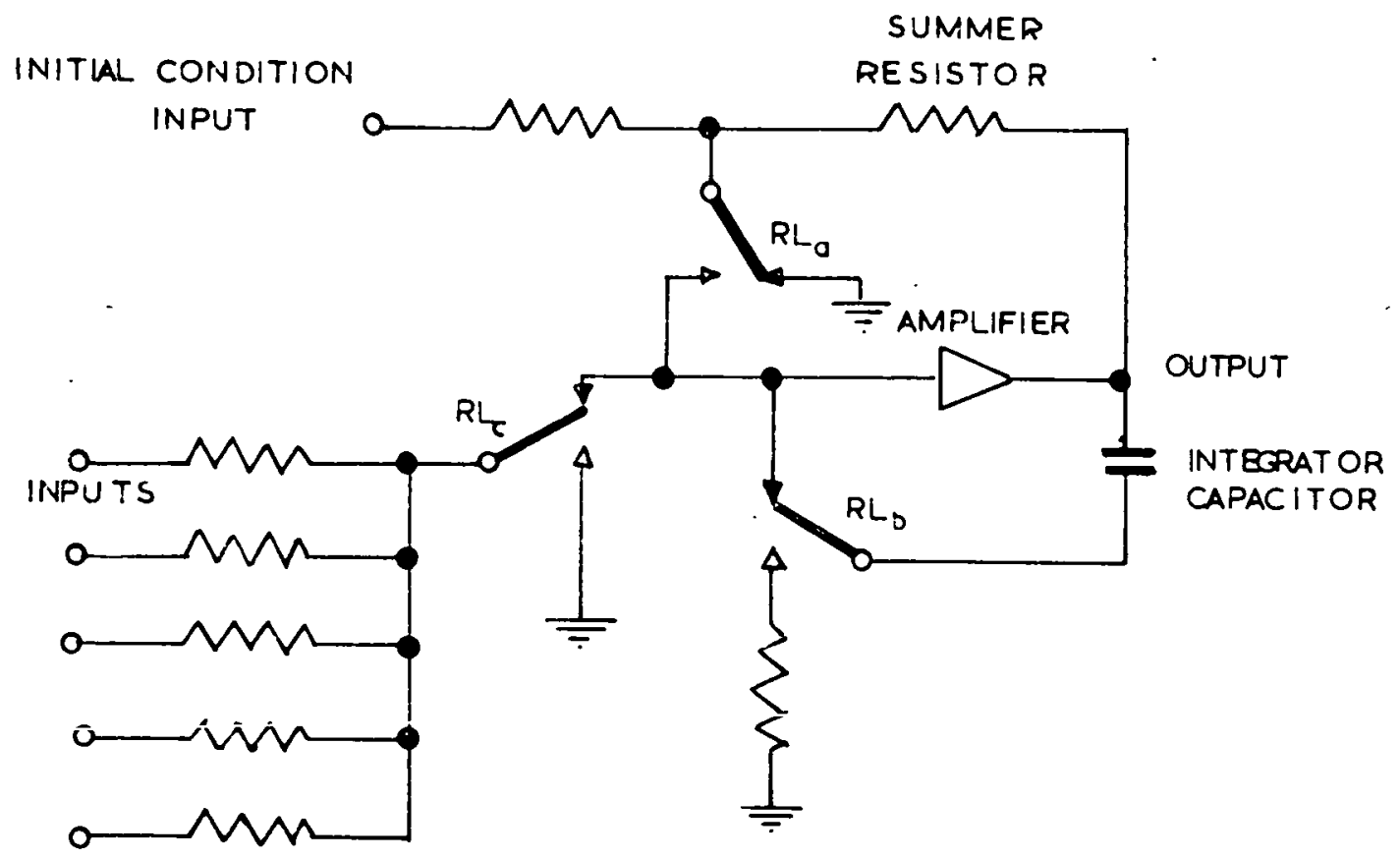

RELAYS SHOWN IN DE-ENERGISED POSITION

( circuit irom Solortron 247 manual)

SIMPLIFIED SUMMER-INTEGRATOR DIAGRAM

FIG $4: 2$ 


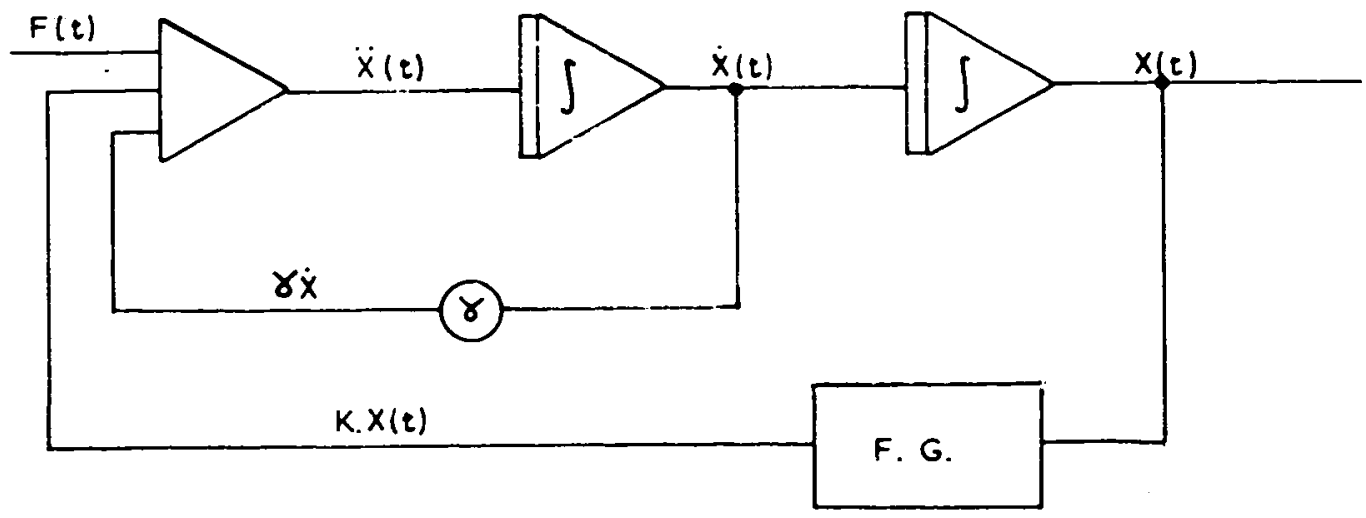

Simplified Diagram of Basic Computer System

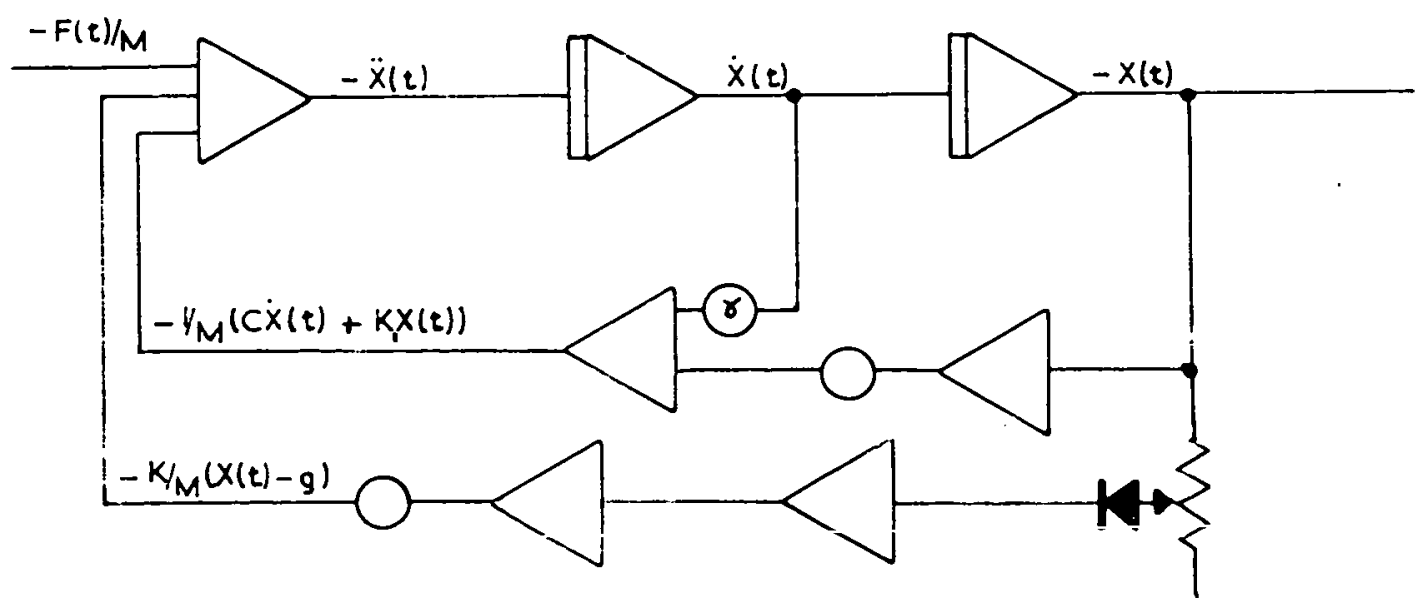

$$
M \ddot{x}+C \dot{x}+K(x)=F(t)
$$

SIMPLEST COMPIJTER CIRCUIT

FIG. 4: $\mathrm{CI}$ 


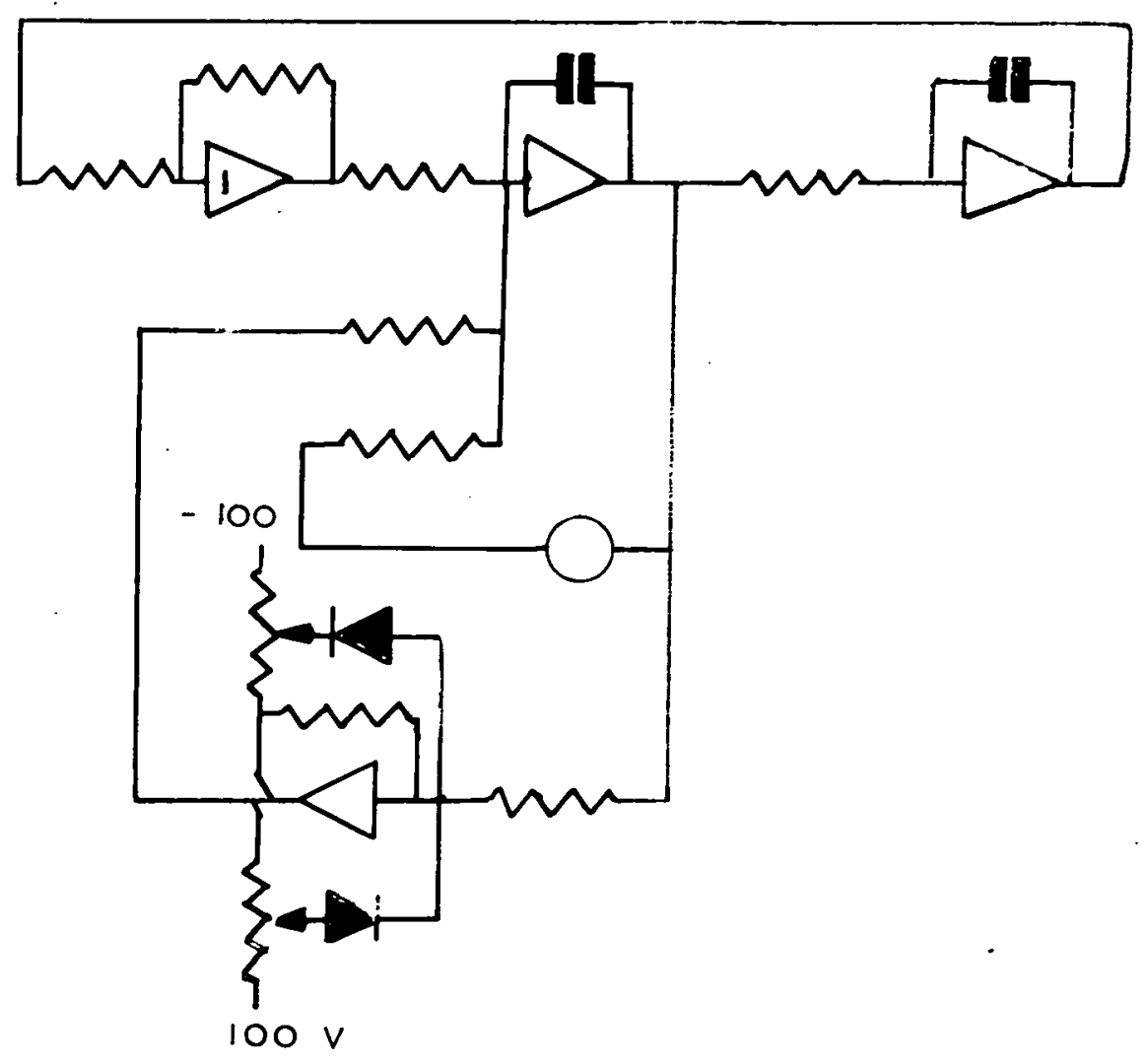

SINE waVE generator AMPLITUde STABILISED C!RCU!T FPOM SOLARTROA 24? MAAUIJAL 
required to solve $a \ddot{x}+b \dot{x}+c x=F(t)$ is shown in Fig. ${ }^{4} c_{0.1}$. The discontinuous function of spring stiffness is generated using a diode. Fig. 4.3 shows a circuit for generating a sine waveform. The symbols F.G. represent any function generator, the exact nature of which will be explained in the text describing that particular circuit.

\subsubsection{Setting up and scaling}

Since the computer solves differential equations by integration (differentiation being unstable) the equation is written as

$$
\ddot{x}=f(t)-a x-b\langle x-g\rangle-c \dot{x}^{I} \quad \ldots 4.1
$$

and the amplifier line diagram is drawn fig. 4.3. Writing the equation again with the usual symbols we have

$$
\ddot{x}=\frac{\hat{F}}{M} \sin \omega t-p_{1}^{2}-p_{2}^{2}\langle x-g\rangle-\gamma_{\dot{x}} \quad \ldots 4.2
$$

A discussion of computer accuracy will appear elsewhere. The basic principle of scaling is that accuracy is improved if all outputs are kept as large as possible without exceeding \pm 100 volts at which value the amplifiers will saturate. Experience showed that this was not very important as long as signals were kept sensibly large, and in general signals were kept at values compatible with easy transcription into physical units. The piece-wise linear nature of the spring function greatily facilitated scaling. In the system a maximum amplitude of .5", a maximum velocity of above $10 \mathrm{ins} / \mathrm{sec}$. , and a maximum acceleration of about three times that of gravity were expected.

\footnotetext{
I Note - the symbol ' $g$ ' used here to represent spring gap setting should not be confused with ' $g$ ' the acceleration of gravity. In general the meaning will be clear from the text.
} 


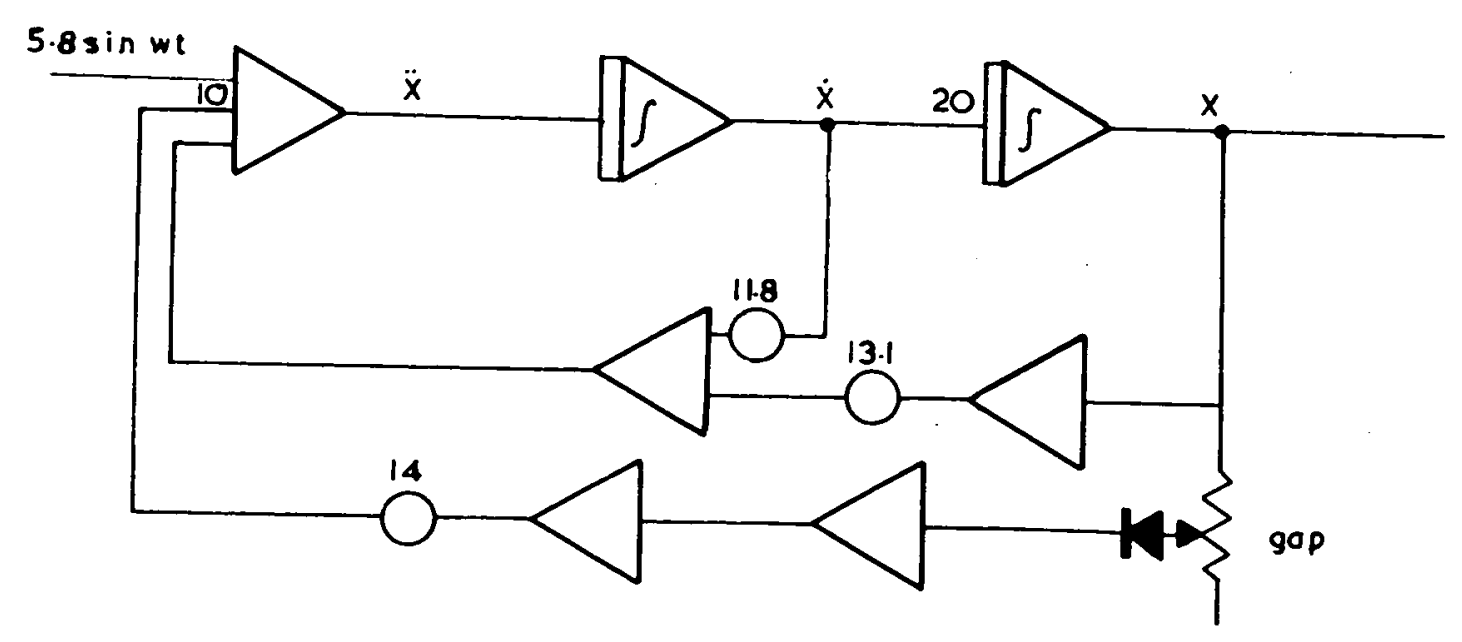

Coefficients set for system A

FIG . $4: C 2$ 
The scaled units were thus

$$
\begin{array}{ll}
\text { acceleration } & I \text { volt }=10 \mathrm{ins} . / \mathrm{sec}^{2} . \\
\text { velocity } & I \text { volt }=1 \mathrm{ins} . / \mathrm{sec} . \\
\text { displacement } & I \text { volt }=.005 \mathrm{ins} .
\end{array}
$$

If due to overloading, it was desired to change these by a factor of two, say, it was only necessary to alter the gap setting and input force.

Having scaled the problem for amplitude another complication

appeared. In this particular computation the table motion was required as exact waveform traces of velocity, acceleration and displacement, from which the slider travel rate could be obtained. The pen recorder available however, while accurate for sinusoidal inputs up to $15 \mathrm{c} / \mathrm{s}$ the required upper limit exhibited considerable overshoot with the non-linear waveforms, and it was thus necessary to slow down the computer to a time base of ten times real time. This slowing down was achieved by keeping all scale factors constant and reducing integrator gains by 10. If the forcing function was being generated outside the computer the time base of the oscillator had to be increased by 10 times also. Fig. C.2 shows the system scaled for the parameters of System A which are tabulated in Appendix A.

\subsubsection{Procedure}

The restriction imposed by the lack of multi-rhannel pen recording facilities greatly complicated the procedure.

While any parameters could easily be varied, only those which were controlled in the experimental work were changed, the computer investigation repeating the tests on the physical system. The information required from the computer was, in descending order of importance, an exact velocity trace, acceleration trace and displacement trace for 
each frequency considered.

Having set up the circuit the force signal was connected and tuned to the frequency for investigation. The computer was then switched to 'Compute' and the system left to settle dorm to steady oscillation. Depending on the stability of the solution this could take up to several minutes. When the system was in steady state three traces were taken - velocity, acceleration and displacement. Earh was taken over a period somewhat greater than that of the 'limits cycle in which the motion was periodic, lines of known voltage being superposed on the trace to give a scale. This was repeated at frequency intervals of $\frac{1}{2} \mathrm{cycle} / \mathrm{sec}$. or less over the entire frequency range in which the table made contact with the hard spring. Thus for each spring configuration considered, the output was a long roll of Teledos recording paper with three traces for each frequency. In common with the experimental results, two stable solutions were occaspionally found at a particular frequency. To examine the stability of a particular solution the problem could be given an impulse and the displacement watched as it passed through the transient and settled down again. The existence of more than one solution at a particular frequency will be discussed later, but in general the solutions which co-existed were a subharmonic solution and a harmonic solution.

Having obtained a velocity trace for the table motion, the travel rate of a particle on the table surface was found by drawing lines of slider velocity and integrating the area between slider and table velocity curves as described in Section 2. This is only possible for slider motion with the table vibrating in the horizontal plane 

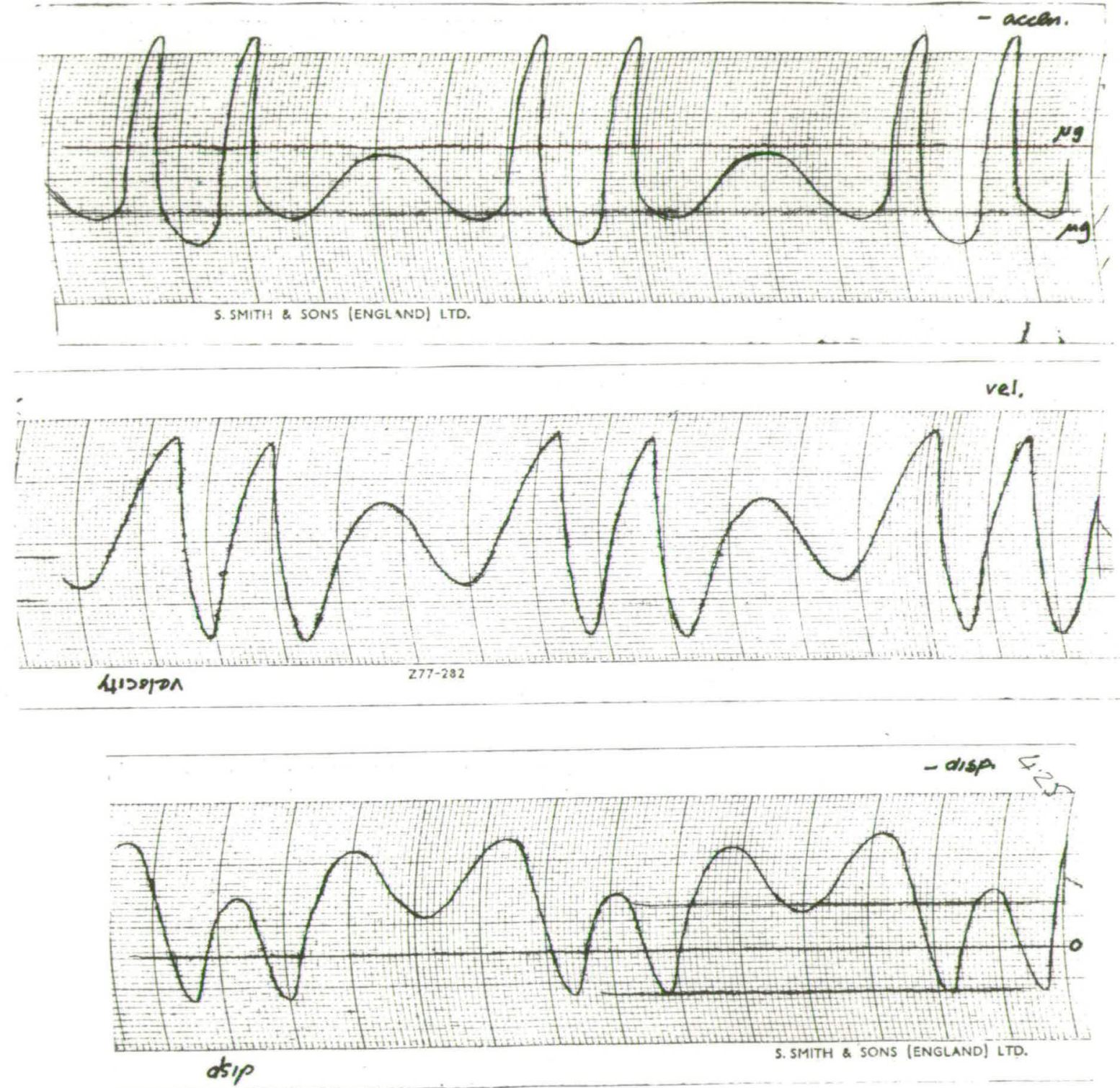

SINGLE CHANNEL RECORDER OUTPUT - PAPER SPEED $2.5 \mathrm{~cm} / \mathrm{sec}$ SYSTEM A gaP $O \quad K_{2} 360$ \& $4.25 \mathrm{c} / \mathrm{s}$ HARMONIC SOLUTION 2 IMPACTS PER CYCLE

Plate 4.I Waveforms obtained from 'Minispace' Analogue Computer using Kelvin and Hughes single channel pen recorder. The horizontal lines superposed on the traces are for scaling purposes. 

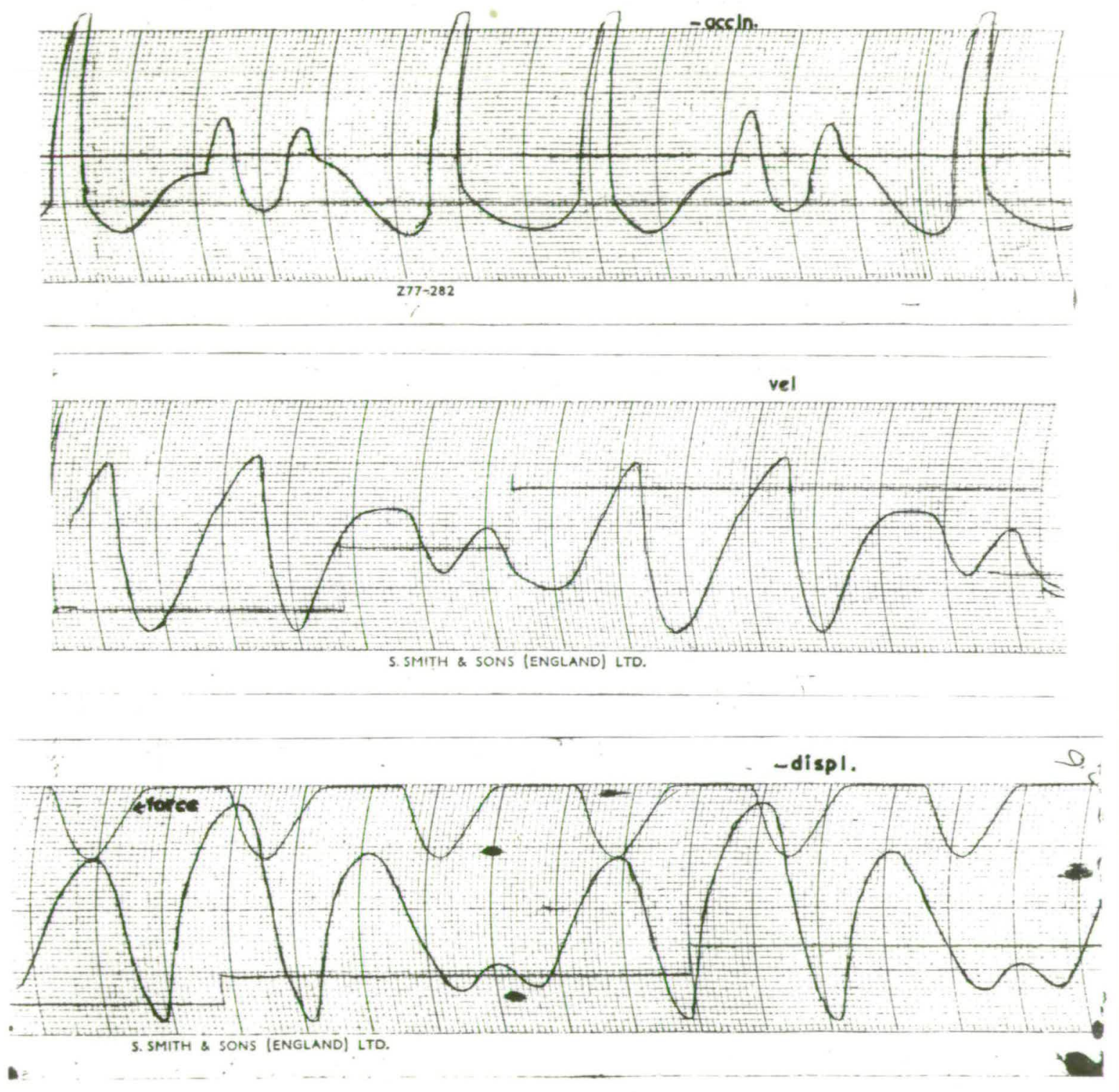

1/3 SUBHARMONIC ANALOGUE COMPUTER RESULT
SYSTEM A GAP Oin. K $360 \mathrm{lb} / \mathrm{in}+1 \mathrm{~g} .25 \mathrm{cls}$ F.75 Ib

Plate $4 e^{2}$ Similar to Plate 4.1 these waveforms show a $\frac{1}{3}$ 'subharmonic'. The superposition of the forcing function in the lower trace is to show the frequency relationship and its phase relationship to the displacement was arbitrarily chosen. 
when the path of slider velocity, when slipping, is a straight line. The pen recorder output was on a curved grid and had to be redrawn onto graph paper. The area between the curves of slider velocity and table velocity was then measured by planimeter. Plate 4.1 shows a set of pen recorder traces for a harmonic solution, and Plate 4.2 is a similar set for a $\frac{1}{3}$ subharmonic solution.

This procedure was most unsatisfactory from a time viewpoint as it took days to obtain travel rates from traces obtained in hours. However within the limits of the accuracy of the method, particularly considering the error introduced by redrawing and using the planimeter, good agreement was found with experimental results and some of the results in section 6 were obtained this way. As a practical means of solution a small system like this is useful for checking regions of stability of solutions. While travel rates can, in time, be obtained for the case of negligible mass on a horizontally vibrating table, it would not be suitable if, for example, the table motion had a vertical component when the slider velocity would no longer be a straight line. To deal with the more complex cases, and also to speed up the time required, a more sophisticated method had to be evolved.

\subsection{The SC 30 and 247 computers}

These are tro machines which can be run quite separately, run in conjunction with each other, or used as a master/slave system. They offer between them a comprehensive range of facilities. Only those which were used will be described in detail.

$4 \cdot 4 \cdot 1$

The $\mathrm{SC} 30$ is a general purpose machine with the following computing equipment. 

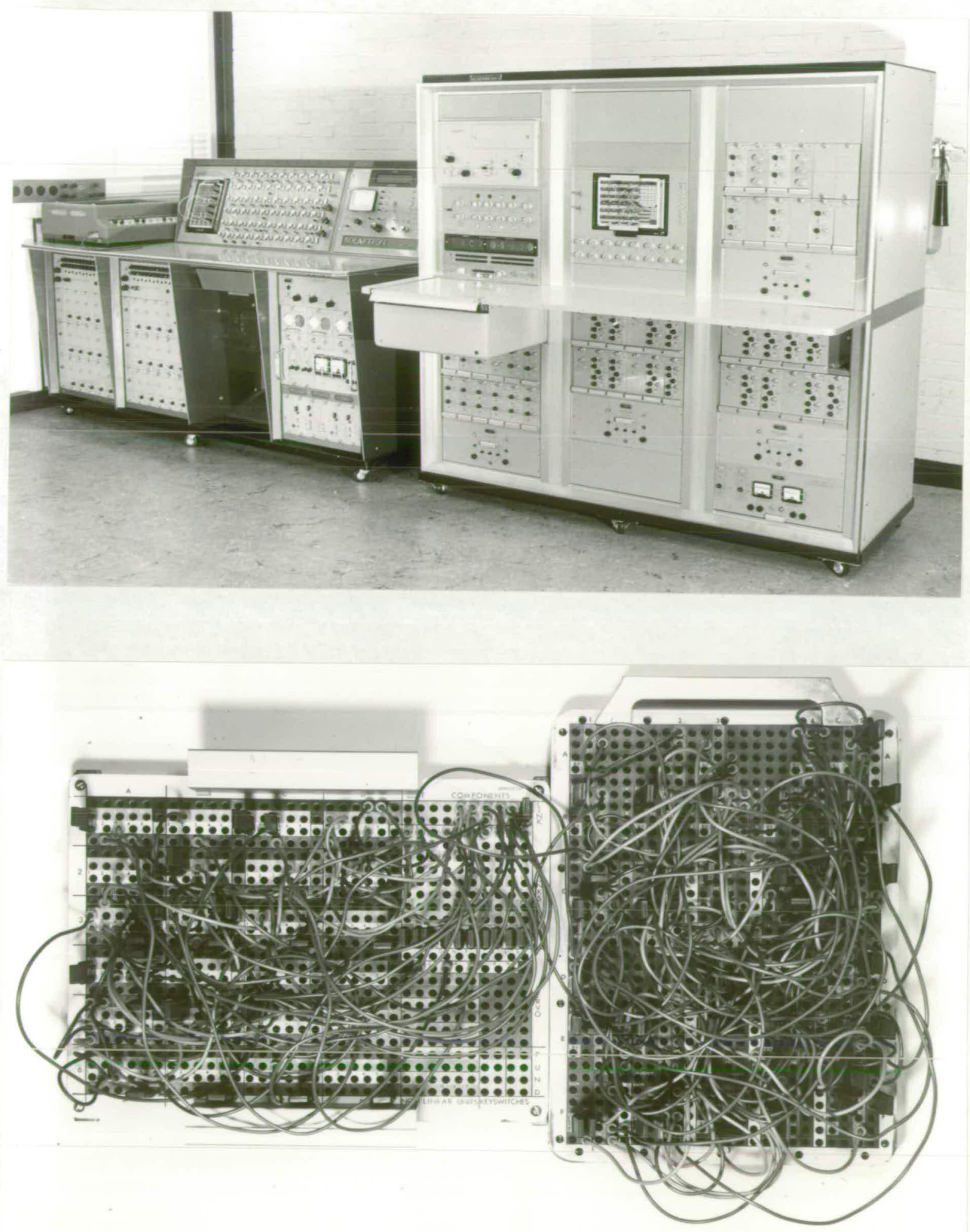

Plate 4.3 (top) Solartron Analogue Computers SC 30 (left) and 247 (bottom) Patch panels for SC 30 (right) and 247. 
60 Coefficient Potentiometers; 30 Operational Amplifiers (10 summers, 20 summer/integrators); 4 Servo-multipliers; 2 General-purpose diode function generators; 4 Symetrical diode limiter bridges; 4 Relay amplifiers. The circuit is wired on a removable patch panel. In the problem considered the servo-multipliers and general purpose diode function generators were not used.

The coefficient potentiometers are similar to those used on the 'Minispace'. The amplifiers are slightly different in that the summer groups have three outputs and the summer/integrators offer five outputs. Each amplifier.can, by means of relays incorporated in it, have its gain as an integrator increased by ten or decreased by ten if used as a summer. The energising 24 volt supply can either be supplied to each individually, or, by means of a trunk connection, any or all amplifiers can be changed by a single link connection. There are no 'free' potentiometers as in the 'Minispace', discontinuous functions being generated by the diode function generator. The limiters are used in conjunction with amplifiers to give a 'soft' limiter - i.e. a gain of unity until the input reaches a preset value, at which level the output is held approximately constant for all higher inputs. Considerable use was made of the relay amplifiers. These allow a signal to be switched in either of two ways depending on the sign of an input signal. A further facility used was one by which any integrator may be switched between 'Problem Check' and 'Compute' states independently of the in control panel. The significance of this will be discussed later. There is an 18 line trunk connection between the SC 30 and 247 machines.

The 247 is the more recent of the two machines. It offers on 

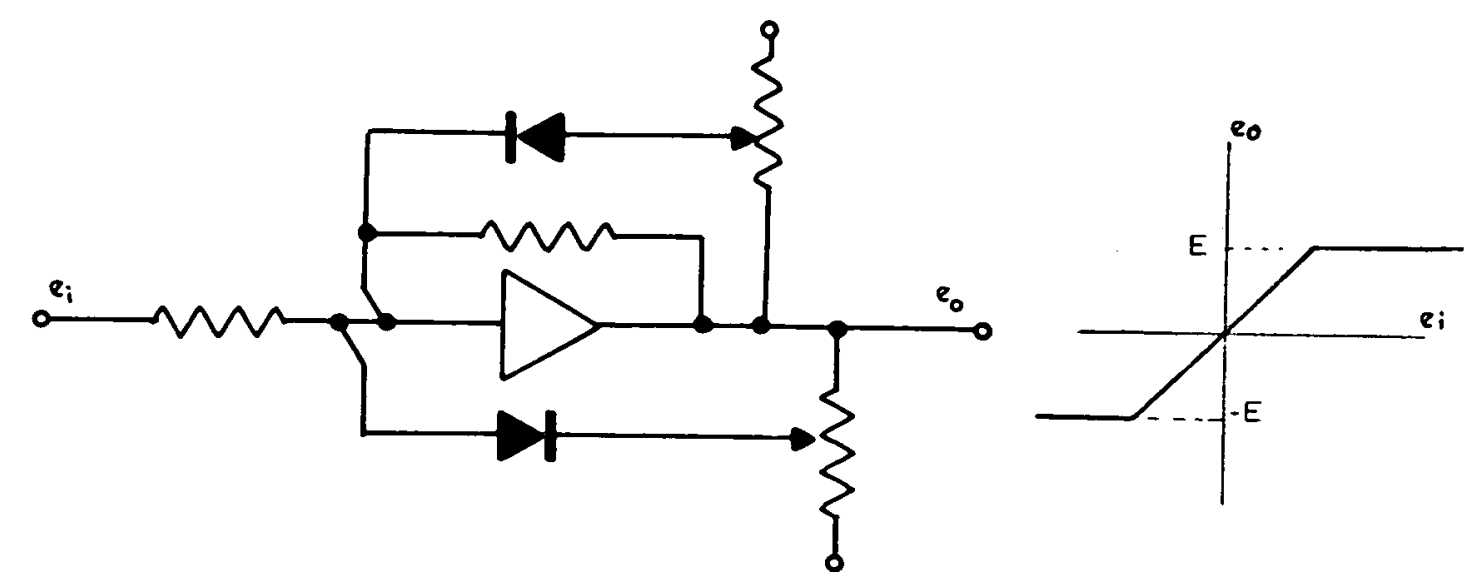

SOFT LIMITER CIRCUIT ON PLUG-IN BOARD
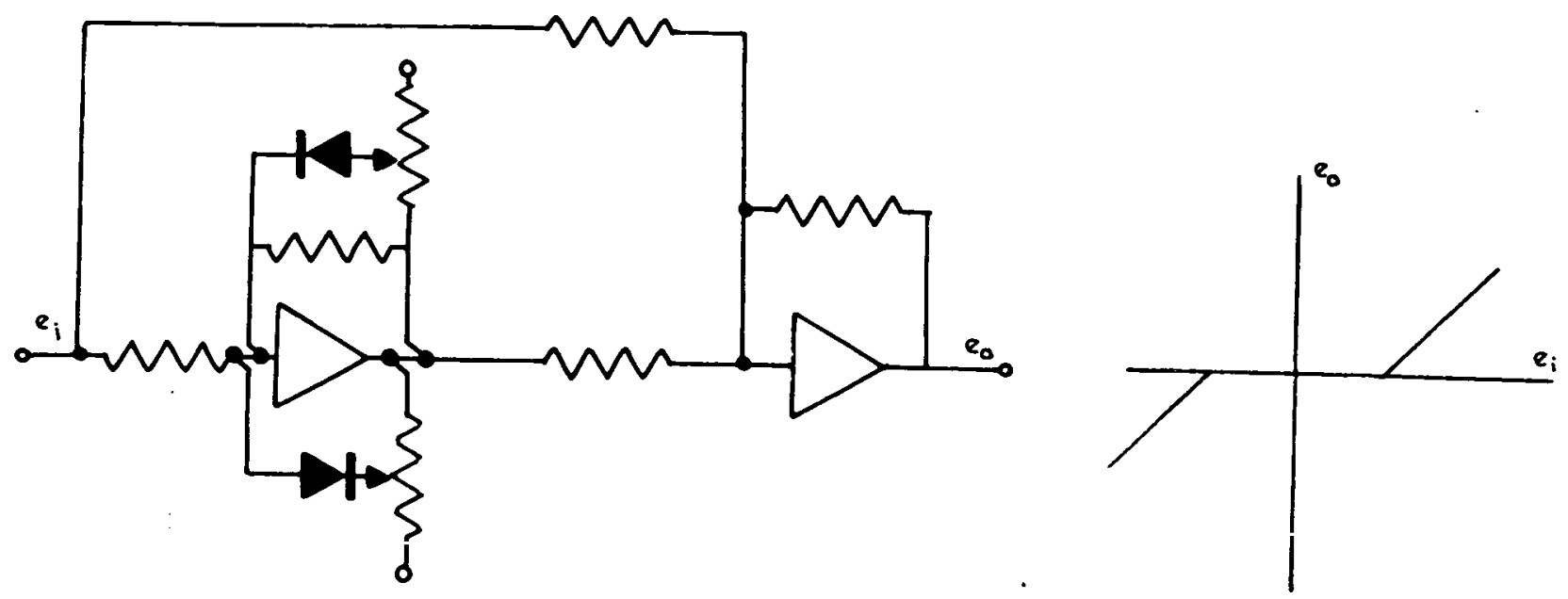

LIMITER USED WITH SECOND AMPLIFIER TO GIVE DEAD ZONE

FIG. $4: 4$ 
a removable patch panel 16 summer/integrators and 8 summer groups, as well as servo-multipliers and various non-linear elements. Of the 48 coefficient potentiometers, 8 are earth free. 5 free diodes have been incorporated. Through the rear of the machine access is obtained to circuit cards associated with each amplifier. For the 8 summer groups a small 'library' of circuit cards is available. In this way hard and soft limiters can be obtained and reed relays incorporated. The latter are a more sophisticated version of the relays on the SC 30 giving quicker switching and less electrical noise. The 247 machine also incorporates a timer allowing computation to proceed for an exactly preset interval. Digital print-out of amplifier output can be obtained automatically on an electric type-writer using a printer unit in conjunction with the timer.

\subsection{The operating states of a computer}

There are four basic operating states of an electronic analogue computer - 'Pot Set', 'Problem Check', 'Compute' and 'Hold'. They are selected by switches on the control panel. These states have been mentioned above at various points and are here discussed in more detail.

'Pot Set', or more explicitly, 'Potentiometer Set' is the state which should be used when the potentiometers are being set up. In this state all integrators are held in their initial condition state, all

summer outputs are 'Problem Check'. In this state all summers operate normally, that is, their input signals are connected to the amplifier terminals. Integrators remain in the Initial Condition state, and all other units operate normally. The initial conditions on integrators are 
set in this state by supplying a voltage to the I.C. terminal and monitoring the output. This is necessary since the initial condition setting resistors are neither precise nor even nominally equal. 'Compute' is the normal operating condition with all units operating. 'Hold' is similar to the compute state except that all integrator input signals are disconnected and grounded. Each voltage is therefore held stationary at the voltage reached as the hold state is entered. 4.5 .1

Now to enlarge on the Integrator External Control facility of the SC 30, briefly mentioned in 4.3.1. On the patch panel of the $\mathrm{SC} 30$ are two holes labelled RXI and RX2. They are associated with the facility whereby an integrator may be switched from the 'Problem Check' to the 'Compute' states by means of external contacts independently of the main control panel. By removing a panel at the rear of the machine access is gained to a set of sockets which enable any integrator to be operated either from the control panel, from line $R X 1$, or from line RX2. An integrator on one of the RX line controls is switched between 'Problem Check' and 'Compute' according to whether the control line is energised (by a 24 volt relay supply) or not. Thus the output of an integrator controlled in this way will either be the voltage at the initial condition terminal (Problem Cheok state) or the voltage at the initial condition at the instant of entering the 'Compute' state together with a voltage which is the integrated input voltage. 
4.6 The computer circuit for horizontal table motion when the slider has negligible mass

This is the case with the most simple mechanism of slider transport as has previously been described in 4.2. The table motion was obtained from the circuit described in 4.2.1 and 4.2.2 with the difference that the diode and free potentiometer used to simulate the discontinuous spring characteristic was replaced by a relay amplifier. This method of generating the discontinuous function (which was not available on the 'Minispace') is to be preferred, as it provides a very sensitive switch compared with a diode which may have a threshold level before it conducts, those incorporated in the 247 requiring $\frac{1}{2}$ volt before conducting. Having obtained the solution to the table motion, the problem was to obtain, within the machine, the slider motion. The theory of the motion and the equations governing it have been covered in section 2, but the main points will be briefly reviewed.

The differential equation of table motion is $M \ddot{x}+c \dot{x}+k(x)=f(t)$. The slider will begin to slip if the horizontal acceleration of the table $(\ddot{X})$ exceeds $\pm \mu \cdot g$, and will continue to slip until its velocity equals that of the table. The condition of table acceleration initiates sliding only if the slider and table are relatively at rest, the auration of tine period of slip being determined by the paths of table and slider velocity.

We now define a 'control function' $G(t)^{I}$ which was used to

I

As is described in the text following $G(t)$ is composed of $h$ two signals $G_{a}(t)$ and $G_{v}(t)$

When slipping is taking place $G_{V}(t)=C$. sign $(X-\dot{X})$. With no slipping $G_{y}(t)=0$. $G_{a}(t)=0$ unless $|\ddot{X}(t)|>g$ when it has a value D. $\operatorname{sign}(\ddot{X}(t))$. C is greater that $D$. 


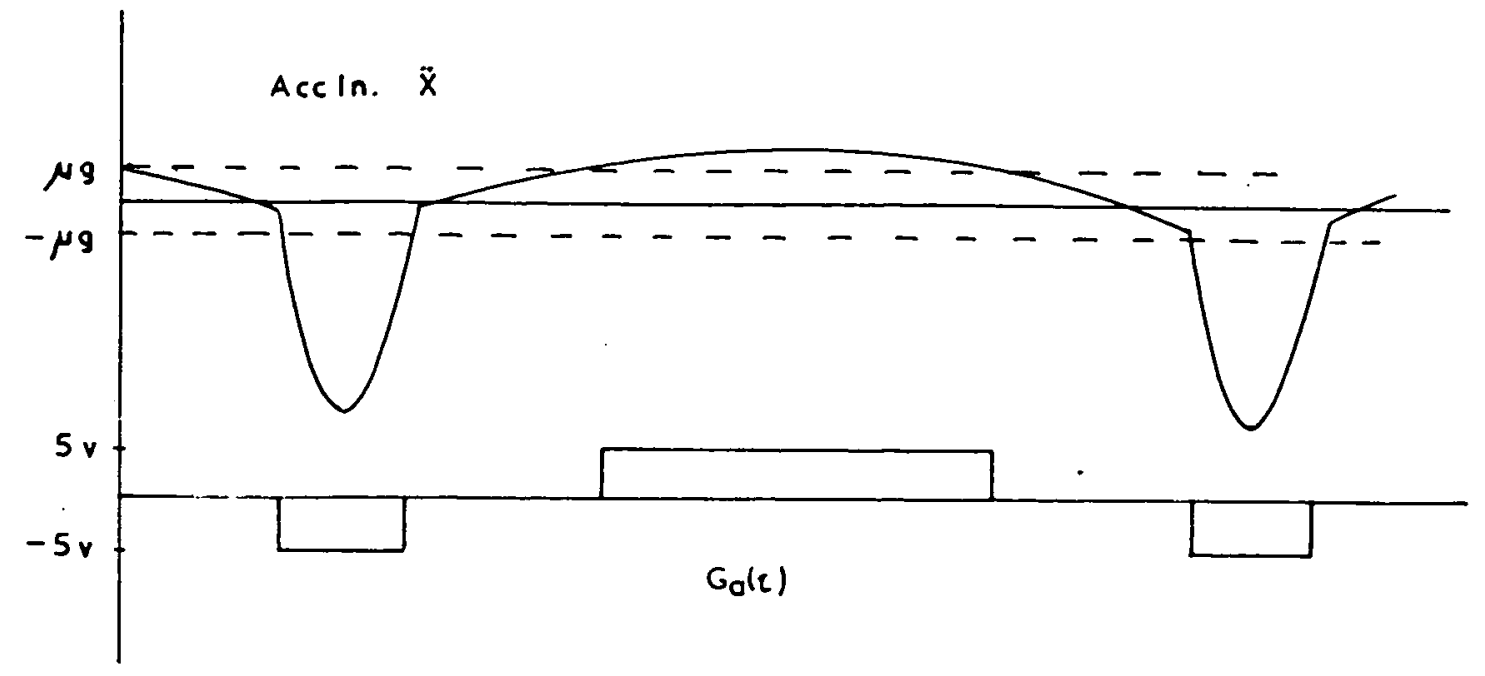

(A)
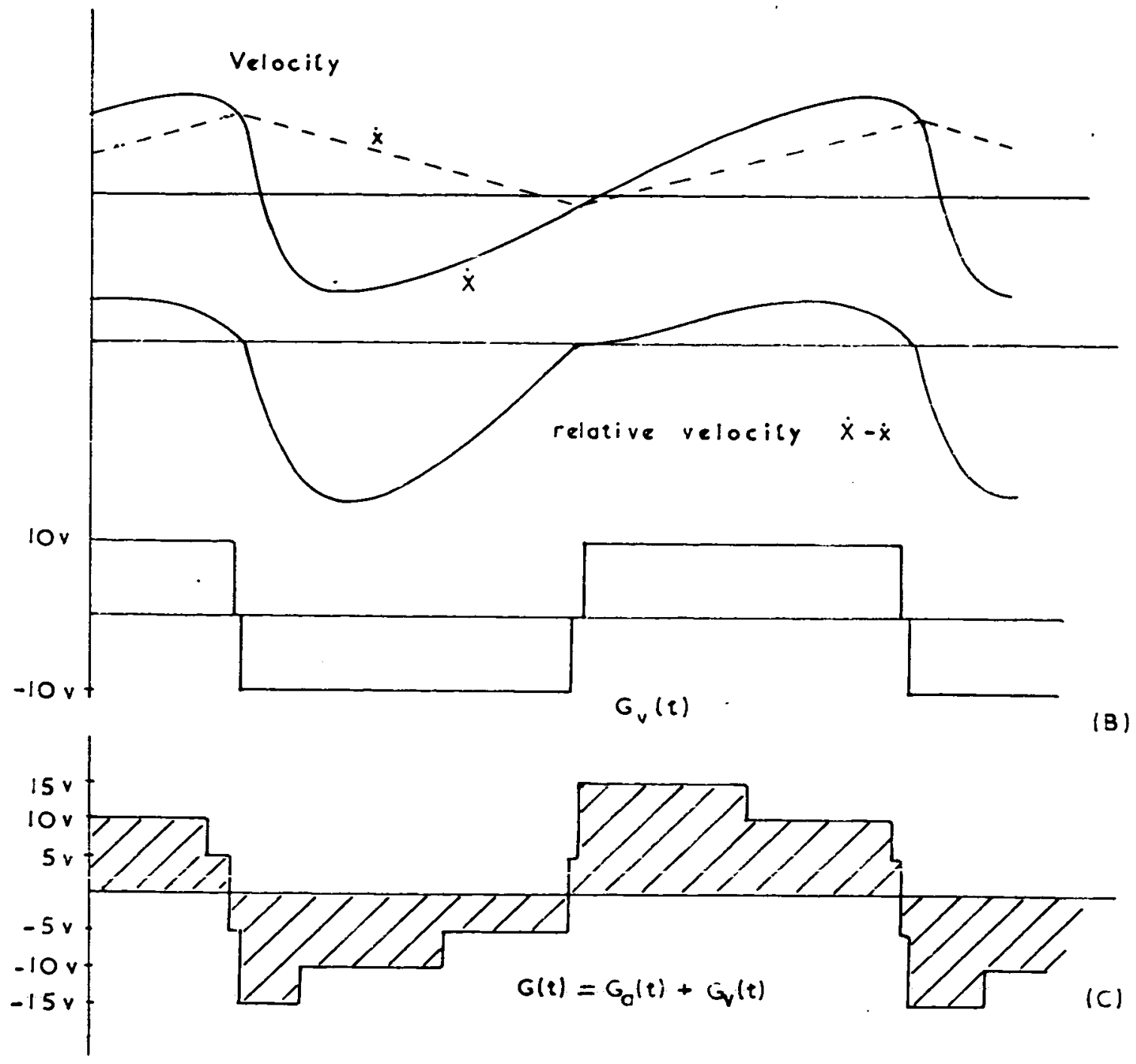

CONSTRUCTION OF CONTROL FUNCTION $G(t)$

F!G. $4: 5$ 
define the regions of slider motion in the analogue computer, as

$$
\begin{array}{ll}
G(t)=0 & \text { all } t \text { except regions defined below } \\
G(t)>0 & t_{0}<t \leq t_{I} \\
G(t)<0 & t_{2}<t \leq t_{3}
\end{array}
$$

where $t_{0}, t_{1}, t_{2}, t_{3}$ are found from

$$
\begin{array}{ll}
\ddot{X}\left(t_{0}\right)=-\mu g ; & \dot{x}\left(t_{1}\right)=\dot{X}\left(t_{1}\right) \\
\ddot{X}\left(t_{2}\right)=\mu g ; & \dot{x}\left(t_{3}\right)=\dot{X}\left(t_{3}\right)
\end{array}
$$

and the slider motion is defined as

$$
\begin{array}{ll}
\dot{x}=\dot{X} & G(t)=0 \\
\dot{x}=\dot{X}\left(t_{0}\right)-\mu g t & \dot{G}(t)<0 \\
\dot{x}=\dot{X}\left(t_{2}\right)+\mu g t & G(t)>0
\end{array}
$$

The above definitions allow that $G(t)$ may have an instantaneous sign change if $t_{0}=t_{3}$ or $t_{1}=t_{2}$ which are conditions for continuous slip. If $t_{0} \neq t_{3}$ or $t_{1} \neq t_{2}$ we have stick slip motion. The problem of the computer circuit was firstly to generate this control function which is essentially, the output from a three way switch.

It will be convenient to refer to the circuit diagram, fig. ${ }^{4} \mathrm{C} .3$ with $\beta=0$ (which folds out for easier reference), and fig. 4.5 in conjunction with the text.

$G(i)$ was composed of tiwo Iunctions, one an acceieration signai which was used to start sliding $G_{\mathrm{a}}(t)$, and the other a signal generated from the voltage representing the relative velocity of the slider and the table $\mathrm{G}_{\mathrm{v}}(t)$. The acceleration waveform was put as the input to a 'dead zone' circuit (see fig. 4.4) whose limits were $\pm \mu$. This circuit gives no output if $|\ddot{x}|<\mu g$. If $\ddot{x}>\mu$ g the output voltage represents $\ddot{x}-\mu g$ and if $\ddot{x}<-\mu g$ the output represents $\ddot{x}+\mu g$. 
This output was then put through a Iimiter circuit to be converted as nearly as possible into the waveform of fig. $4.5 \mathrm{~A}$, which gives an acceleration control signal waveform of -5 volts if $\ddot{x}<-\mu g$, 0 if $|\ddot{X}|<\mu \mathrm{g}$, and +5 volts if $\ddot{x}>\mu g$.

A similar circuit was used with the voltage representing the relative velocity of the slider and the table as its input. In this case the dead zone was set with very small limits, those inherent in the diodes being sufficient, and the limiter was set to give a maximum output of \pm 10 volts, the output signal being as shown in fig. $4.5 \mathrm{~B}$.

Fig. 4.5C shows these signals combined to give $G(t)$. There was no output from either circuit if no slipping is taking place. The 5 volt signal from acceleration started slipping and was immediately augmented by a 10 volt signal from the relative velocity trace. If while slipping was occurring the acceleration condition for slip in the opposite direction was met, the resulting 5 volt signal was drowned by 10 volts from the relative velocity trace until this was zero.

It can be seen that the generation of $G(t)$ made very considerable use of limiter circuits, these being the only easy method of achieving the $+a, 0,-a$ output required. The limiter cards available were 'soft' limiters, i.e. a gain of unity when conducting until the 'stop' was reached at which value the output is held only very approximately constant. Attempts were made to overcome this using 'hard' limiters specially purchased from Solartron Ltd., but these were too inflexible for our requirement (being single sided and having a preset limiting level of 100 volts). To achieve the sharp pulses required two limiters were used in series. While the reason for the dead zone of $\pm \mu \mathrm{g}$ in the case of the acceleration is obvious there is no 
physical nesissity. for a dead zone in the path of relative velocity. This was introduced to improve stability and to avoid small errors in the relative velocity signal from starting or continuing slider motion. The introduction of the dead zone, requiring a relative velocity of .01 ins./sec. before the signal was used in $G(t)$ did not introduce any error, for at the instant of starting to slip, acceleration is the dominant control and remains so for a short time after slipping has begun. Wille the diodes have this $\frac{1}{2}$ volt threshold level before conducting, they continue to do so as long as the input is positiffe so no error is involved.

The control function $G(t)$ depended on the availability of a voltage representing the relative velocity of the slider and table, which in turn required a voltage of slider velocity. This latter was produced using $G(t)$ as the control function. The slider velocity must be identical to the table velocity for no slipping, have a positive or negative gradient of $\mu \mathrm{g}$ when slipping, and be capable of instantaneous sign change for continuous slip motion. To achieve this the external integrator relay control facility on the $\mathrm{SC} 30$ was used. Following the circuit diagram $C .3$ the control function $G(t)$ was used as an input to two relay amplifiers, one of which was in its normal position (arm connected to 'positive' terminal) and the other having a small negative signal input to cause the arm to be connected to the negative terminal. A 24 volt relay supply voltage was put onto the arm of the relay amplifiers, and $\mathrm{RXI}$ and $\mathrm{RX} 2$ (the two integrator relay control lines) energised from the terminals to which the relay amplifiers arms were connected for zero input. The integrator control lines were each used to control the operating states of one 
integrator as described in 4.4.1. When $G(t)$ was zero, $R X I$ and $R X 2$ were both energised by a 24 volt relay supply and both integrators were held in the 'Problem Check' state. If $G(t)$ became positive, control line $\mathrm{RX} 2$ was no longer energised by 24 volts and its associated integrator entered the 'Compute' state while the other remained in 'Problen Check'. Similar switching occurred if $G(t)$ became negative. The integrator controlled by $\mathrm{RXI}$ had a voltage representing $+\mu \mathrm{g}$ as its input, with $-\mu \mathrm{g}$ volts as the input to the integrator controlled by RX2. Both integrators had a voltage $\dot{X}$ (the table velocity) as input to their initial conditions. The outputs from these integrators were connected to the input of a summer together with the signal of table velocity, the latter amplified by a factor of 2. The setting up of the initial conditions on the integrators had to be most accurately carried out so that in the 'Problem Check' state the output was exactly the input voltage (with a sign change).

When the circuit was set in the 'Compute' state at the main control panel the integrators controlled by the lines $R X I$ and $R X 2$ remained in the 'Problem Check' state as Iong as $G(t)$ was zero. Their output was then minus the table velocity, and the output from the summer was zero. If now the acceleration exceeded $+\mu \mathrm{g}, \mathrm{G}(t)$ was no longer zero and the arm of the relay amplifier connected to line RXI changed over. The integrator controlled by RXI then went into 'Compute' when its output was the instantaneous voltage at the initial condition at the moment of entering the 'Compute' state together with the integrated input voltage ie. $-\left(\dot{x}_{0}+\mu g t\right)$. The output from the integrator associated with RX2 was $-\dot{X}(t)$ and hence the output from the summer following the integrators was the relative velocity $\dot{X}(t)-\left(\dot{X}_{0}+\mu g t\right)$. 
As soon as the relative velocity was no longer zero it was used to generated part of $G(t)$ as described above. It can be seen that this circuit allows for a stable position with no slip taking place, single sided slip, double stick/slip or continuous slip when an instantaneous change in the sign of $G(t)$ causes both integrators to change their operating states simultaneously.

The system described above was felt to be the best possible to give the slider motion. However, although relay switching times were fast, it was found necessary to slow down the time base of the computer to 100 times real time to give accurate instantaneous velocity changes in continuous slip motion. The accuracy of the voltage representing relative velocity depended on the abllity of the output of an integrator to follow accurately a dynamic signal at its initial condition input terminal with no phase lag, and the speed of response of the integrator When changing from 'Compute' to 'Problem Check'. The relative importance of these effects was not investigated but some inaccuracy was undoubtedly present.

By Integrating the relative slider velocity signal a trace of relative slider displacement was obtained. Only the solution with the table oscillating in steady state was of interest, when the relative displacement of the table and slider over a known interval was required. Since relative displacement was a function which increased continuously with time as the table motion passed through the transient state, the relative velocity could not be integrated over the entire computing period without the integrator amplifier overloading. To allow separate control of this one integrator the main computation, and in particular all other integrators, were set up on the SC 30 , the 247 having only 
elements such as summers, limiters and relay amplifiers which function normally in the 'Problem Check', 'Compute' and 'Hold' states. The computers were not used in the full master/slave relationship in which one control panel governs the operating states of both machines. Instead the 247 was normally in 'Problem Check' when the SC 30 was in 'Compute', the output from the integrator being zero. Once the motion had settled into steady state the 247 was switched to 'Compute' for a known period, and the output from the integrator monitored on the digital voltmeter. The displacement per cycle could either be obtained by taking the successive maximum values displayed or by using the timer to control the period of integration to 100 seconds ( 1 second of real time). The latter method allowed results to be automatically printed out using the printer and electric type-writer unit.

In Fig. C.3 provision is shown for the introduction of Coulomb damping, acting on the table alone $\mathrm{P}$ sign $(\dot{X})$. This is easily obtained using a relay amplifier. The circuit shown does not make provision for $\dot{X}=0$, since for zero input the relay amplifier has a positively switched output. The circuit is thus not suitable for table motions of the stick/slip type, but otherwise no error is involved since $\dot{X}=0$ only instantaneously.

4.7 The circuit for the case of a slider of negligible mass on a horizontal table vibrating in a plane at po to the horizontal

The circuit for this problem was a very slightly modified version of that used in the previous section. Fig. C.3 shows the circuit with $\beta \neq 0$ for this case.

The equations of motion are described in full in section 2. Since the slider is assumed to have zero mass the motion of the table on its new axis at ${\ell^{\circ}}^{\circ}$ the horizontal is unaffected provided we 
measure the gap setting from the equilibrium position of the table. In a practical system this would require that the gap be set for each value of $\beta$. The table motion then has horizontal and vertical components of motion, $X(t) \cos \beta$ and $X(t) \sin \beta$ respectively. The motion of the slider, when slipping is $\ddot{x}(t)= \pm \mu(g+\ddot{x}(t) \sin \beta)$. The relative velocity is $\dot{X}(t) \cos \beta-\dot{x}(t)$. $G(t)$ the control function is defined as before

$$
\begin{array}{ll}
G(t)=0 & \text { all } t \text { except regions below } \\
G(t)=0 & t_{0}<t \leq t_{1} \\
G(t) \succ 0 & t_{2}<t \leq t_{3}
\end{array}
$$

where $t_{0}, t_{1}, t_{2}$ and $t_{3}$ are defined from

$$
\begin{aligned}
& \ddot{x}\left(t_{0}\right) \cos \dot{\beta}=-\mu\left(g+\ddot{x}\left(t_{0}\right) \sin \beta\right) ; \dot{x}\left(t_{1}\right)=\dot{x}\left(t_{1}\right) \cos \beta \\
& \ddot{x}\left(t_{2}\right) \cos \beta=\mu\left(g+\ddot{x}\left(t_{2}\right) \sin \beta\right) ; \dot{x}\left(t_{3}\right)=\dot{x}\left(t_{3}\right) \cos \beta
\end{aligned}
$$

and

$$
\begin{aligned}
\dot{x}(t) & =\dot{x}(t) \\
& =\dot{x}\left(t_{0}\right) \cos \beta-\mu^{\prime}(g+\ddot{x}(t) \sin \beta) t \quad G(t)>0 \\
& =\dot{x}\left(t_{2}\right) \cos \beta+\mu^{\prime}(g+\ddot{x}(t) \sin \beta) t \quad G(t)<0
\end{aligned}
$$

The method used to generate $G(t)$ was similar to that used for the case of $\beta=0$. The input condition to the initial condition of the integrators on external relay control was multiplied by cos using a potentiometer, and the input to these integrators has an additional term of $\pm \mu \ddot{x}(t) \sin \beta$ as shown in fig. 0.3. The dead zone used to generate the acceleration component of the control function had to be modified so that it gave an output when the input signal multiplied 
by $\cos \dot{\beta}$ exceeded $\pm \mu(g+\ddot{x}(t) \sin \beta)$. This was done by replacing the 100 volt referenos applied to the 'high' end of the free potentiometer by $\mu(g+\ddot{X}(t) \sin \beta)$ and multiplying the input signal by cos $\beta$ using a potentiometer. In all other respects the circuit was identical to that previously described in 4.5 .

\subsection{Generation of forcing function}

As previously mentioned there are two means of generating the force input to the problem - either by an external oscillator or by a sub-circuit within the computer. In the case of the 'Minispcce' the argument against generating the signal in the machine was that too many amplifiers were required. This however, was not the case for the joint SC 30-247 system. The main consideration was which method would give the quickest solutions, bearing in mind that there was light damping in the system, and that while slowing down the computer to a time base of 10 times real time (and later 100 times) did not increase the 'problem' time to steady state, the operator had to wait minutes of 'real' time. Using the frocility for changing integrator gains via the trunk line with the forcing function generated in the computer, the machine could be run at a faster time until an approximately steady state was obtained. If then the machine was put into :Holdi, tine time bases of aII integrators reduced by ten and the computer returned to 'Compute', the computation continued at the new machine time without any perturbation of the solution due to the changed time base. If this same approach was tried with an externally generated signal, only the computer circuit was held while the integrator gains were changed. The perturbation resulting from any phase shift between force and motion when the computation was 
restarted destroyed the solution previously obtained. The advantage of this latter system were that one solution was used as the basis of the next computation. This is because the amplitude and frequency of the forcing function can bo set by calibrated dials and this can be done with the computers in the 'Compute' state. This gives quicker convergence to steady state for successive cases compared with generating the forcing function within the machine. Since the sine wave generator solves $\ddot{x}+\omega^{2} x=0$ to obtain $x=P$ sin $\omega t$ where $P$ is an initial condition and $\omega^{2}$ is set by a potentiometer it is necessary to return to 'Problem Check' or 'Pot Set' to vary P or $\omega$. Best results and greater ease of computation were achieved with an external oscillator.

\subsection{Ancillary Equipment}

On changing from the 'Minispace' to the larger machines a greater range of ancillary equipment was available. A low frequency 'Servomex' wave form generator type II! I4I was used to provide a sinusoidal forcing function. In addition to the monitor facilities provided by two five figure digital voltmeters one incorporated in each unit, and the automatic print-out facility of the 247 there was also available a 4 channel Airmec display oscilloscope type 2'79 and a Bryans Aeroequipment Itd. X $-Y$ Cowrdira te Plotting Table. With funds made available from

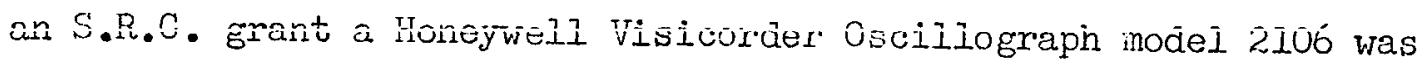
purchased. This ultra-violet recorder gave five recording channels. - The input signal from the analogue computer had to be highly alternated to avoid overloading, and the signal source impedarce had to be matched to the galvanometers used.

\subsection{Procedure}

The experimental procedure was similar to that used in the 
'Minispace'. The parameters of the system were set up and the various sub-circuits balanced as described. The SC 30 was used in the 'Compute' state with the 247 in the 'Problem Check'. Once a steady state solution was obtained the 247 was switched to 'Compute' using the timer control. By this method the integral of the relative velocity over 100 seconds of computer time, corresponding to $I$ second of 'real' time was obtained and displayed on the digital voltmeter. Acceleration, displacement, table and slider velocities were displayed on the display oscilloscope to ensure that there was no obvious fault in the circuit. Records were made using the $U-V$ recorder or $X-Y$ plotter where permanent records of any particular motion were required. As has been discussed there was no change in the circuit controlling the table motion as the angle was varied, but two coefficient potentiometers in the circuit generating slider motion. The settings of coefficient potentiometers could not be monitored with the machine in any state other than 'Pot Set'. However, by marking the potentiometer knob settings on the coefficient potentiometer panel the settings for various values of $\beta$ could be set without monitoring the setting each time. With the circuit in steady state oscillation all angles could then be achieved quickly and values of travel rate were obtained for all angles considered for a particular spring configuration and frequency without stopping to computation. Using the circuit described all experiments carried out on Systems $A$ and $B$ in the laboratory were repeated. It is obvious that the system, primarily designed for a non-linear spring characteristic, was completely suited to linear systems also. The readings taken were three gap settings of three 'hard' springs ( 9 configurations) for System A which was only for horizontal motion, and the same 
9 configurations, plus the linear case for System $B$ at all values of from 0 to $30^{\circ}$ at $5^{\circ}$ intervals. The results are show graphically and discussed in Sostion 6. The circuits used were not suitable when the maximum vertical acceleration exceeded that of gravity.

4.17 A circuit for a table undergoing horizontal table motion when the mass of the material being conveyed is comparable to the table mass.

In this particular case, which is a very practical one, the transported material rather than a single slider would probably be a bulk medium which moves as a rigid body. When sliding is taking place the material is subject to a constant deceleration of $\pm \mu g$ as before, but in this case the motion of the table is affected by the presence of the material being transported. When there is no relative motion the vibrating system has an effective mass of the table mass plus the mass of the material on the surface of the table. As soon as relative sliding begins the effective vibrating mass of the system becomes that of the table alone. Due to the motion of the slider there is a friction damping force on the table of $\mu \mathrm{mg} \operatorname{sign}(\dot{X}-\dot{x})$ where $m$ is the mass of the material being transported.

Following Section $4.6, G(t)$ is defined as

$$
\begin{array}{ll}
G(t)=0 & \text { all } t \text { except regions below } \\
G(t)>0 & t_{0}<t \leq t_{1} \\
G(t)<0 & t_{2}<t \leq t_{3}
\end{array}
$$

where $t_{0}, t_{1}, t_{2}$ and $t_{3}$ are defined by

$$
\begin{aligned}
& \ddot{x}\left(t_{0}\right)=-\mu g ; \dot{x}\left(t_{1}\right)=\dot{x}\left(t_{1}\right) \\
& \ddot{x}\left(t_{2}\right)=-\mu g ; \dot{x}\left(t_{3}\right)=\dot{x}\left(t_{3}\right)
\end{aligned}
$$

The motion of the table is defined by

$$
\begin{array}{rlrl}
(M+m) \ddot{X}+C \dot{X}+k(X)=f(t) & G(t) & =0 \\
\ddot{M} \dot{X}+C \dot{X}+k(X)+\mu m g \operatorname{sign}(\dot{X}-\dot{X})=f(t) & |G(t)|>0 \\
\ldots & \ldots 4.8
\end{array}
$$


and the slider motion is defined by

$$
\begin{array}{ll}
\dot{x}(t)=\dot{x}(t) & G(t)=0 \\
\dot{x}(t)=\dot{x}\left(t_{0}\right)-\mu g t & G(t)>0 \\
\dot{x}(t)=\dot{x}\left(t_{2}\right)+\mu g t & G(t)<0
\end{array}
$$

It can be seen that in this case there is no change in either the control function $G(t)$ or the equations of slider motion from the circuit described in section 4.6. The main change is that some fairly complicated switching of signals is required in the circuit associated with the table motion. Circuit diagram $\mathrm{C} 5$ shows a circuit for this particular case. Coulomb damping is not included in this diagram.

Before describing the system in detail it is noted that the switching circuit was rather more complicated than that shown. It will also be seen that a quantity used as a signal for computation purposes was obtained from the relay supply voltage (24 volts normally) instead of from the 100 volt reference signal. The circuit shown is a practical means of solving the equations with the facilities available. It is not claimed that it conforms to the best principles of analogue computing.

When no slipping is taking place in the system with a large mass the equation is

giving

$$
(M+m) \ddot{X}+C \dot{X}+k(x)=f(t)
$$

$$
\ddot{X}=\frac{I}{M+m}(f(t)-C \dot{X}-k(X))
$$

or

$$
\ddot{X}=\frac{M}{M+m}\left(\frac{f(t)}{M}-x \dot{X}-\omega_{1}^{2} X-\omega_{2}^{2}<X-X_{g} \dot{y}\right)
$$

The acceleration term was thus multiplied by $M /(M+m)$ when no slipping was taking place, this keeping the other coefficients of the system correct. This multiplication was achieved using a relay 
amplifier, the input to which was the modulus of the control function $G(t)$, the rectification being achieved by yet another relay amplifier. When slipping was taking place the coulomb damping effect of the material requires an input to the acceleration summer of magnitude $\mathrm{jmg} / \mathrm{M}$ and sign controlled by the direction of relative slipping. This voltage was obtained from the voltage available at the previously unused terminals of the relays which energised $R X 1$ and $R X 2$ when slipping was taking place. Since a voltage was available from one or the other depending on the direction of relative slip no difficulty existed in obtaining the correct sign. The relay supply was only nominally 24 volts and voltages could not be obtained from it by setting a coefficient potentiometer as would be done if the 100 volt reference source was used. Instead the required voltage had to be set by monitoring it as an amplifier output. There was associated with the relays energising $R X 1$ and $R X 2$ scme tendency to chatter and also some noise at the terminal not connected to the arm. To prevent this interfering with the system, the signal representing the coulomb damping was further controlled by the relay used to multiply the acceleration output since the relay amplifiers on the 247 allow simultaneous switching of two signals by a single input. This circuit is not shown on $\mathrm{C} 5$.

\subsection{Imperfections in the Analogue Circuits}

The accuracy of the computer system will be evaluated in section 6 after discussion of the results. A few comments will be made however, before concluding this chapter.

One of the main unknowns of the computer accuracy was caused by the breakdown of a voltage regulator and stabiliser in the power supply 
to the machines. This caused a higher voltage than recommended onto the amplifier valves, digital voltmeter etc., and before this was discovered, and for some little time after it was rectified, a fairly regular series of valve breakdowns was found. The effects on readings taken while this was going on are not known. A known source of error was the relay amplifiers of the SC 30 . They give relatively slow switching and there is a lot of 'noise' superposed on the signal. While one was used to generate the coulomb damping term in circuit $\mathrm{C}_{4.3}$, the output from it was not a sharp square pulse of $\pm A$ as one might expect, but a signal which has the 'corners' smoothed due to slow switching and possibly interference from the free terminal. This was not a fault on the relay amplifiers of the 247 .

\subsection{Some further computer circuits}

Remembering that circuit diagram $\mathrm{C} .3$ is a somewhat simplified picture, it can be seen that the computer was being used very near to its capacity. Extension of the circuit to the problem of a slider mass comparable to that of the table with the table vibrating in a plane at $\beta^{\circ}$, involving as it does not one multiplication of acceleration but two, depending on the direction of slip, was considered outwith the capacity of the machine. Similarly the case when the maximum vertical table acceleration exceeds gravity, involving the simultaneous computation of the trajectory of the slider, and switching of further signals was not achieved. One case which would be easy to obtain is that where the table motion is at $\beta^{0}$ to the horizontal and the surface is at $\varnothing^{\circ}$ to the horizontal. This would necessitate an unsymmetrical limit to the dead zone in generating $G_{a}(t)$ and inputs differing by a constant term to the integrators controlling slider motion. One would 
of course have to define whether travel rate was in the horizontal or in the plane of the table surface. If the motion of the table was known, as in the case of a linear non-resonant system, the circuit would be simplified by the removal of the table circuit, though the displacement, velocity and acceleration would be required. This would probably be obtained by using an external oscillator to produce a signal proportional to table acceleration. While the power requirement of the conveyor is unlikely to be important in a resonance system, if a non-resonant system was being used a voltage of the integral $\int F d x$ the work done by the force could be achieved using a servo-multiplier. 


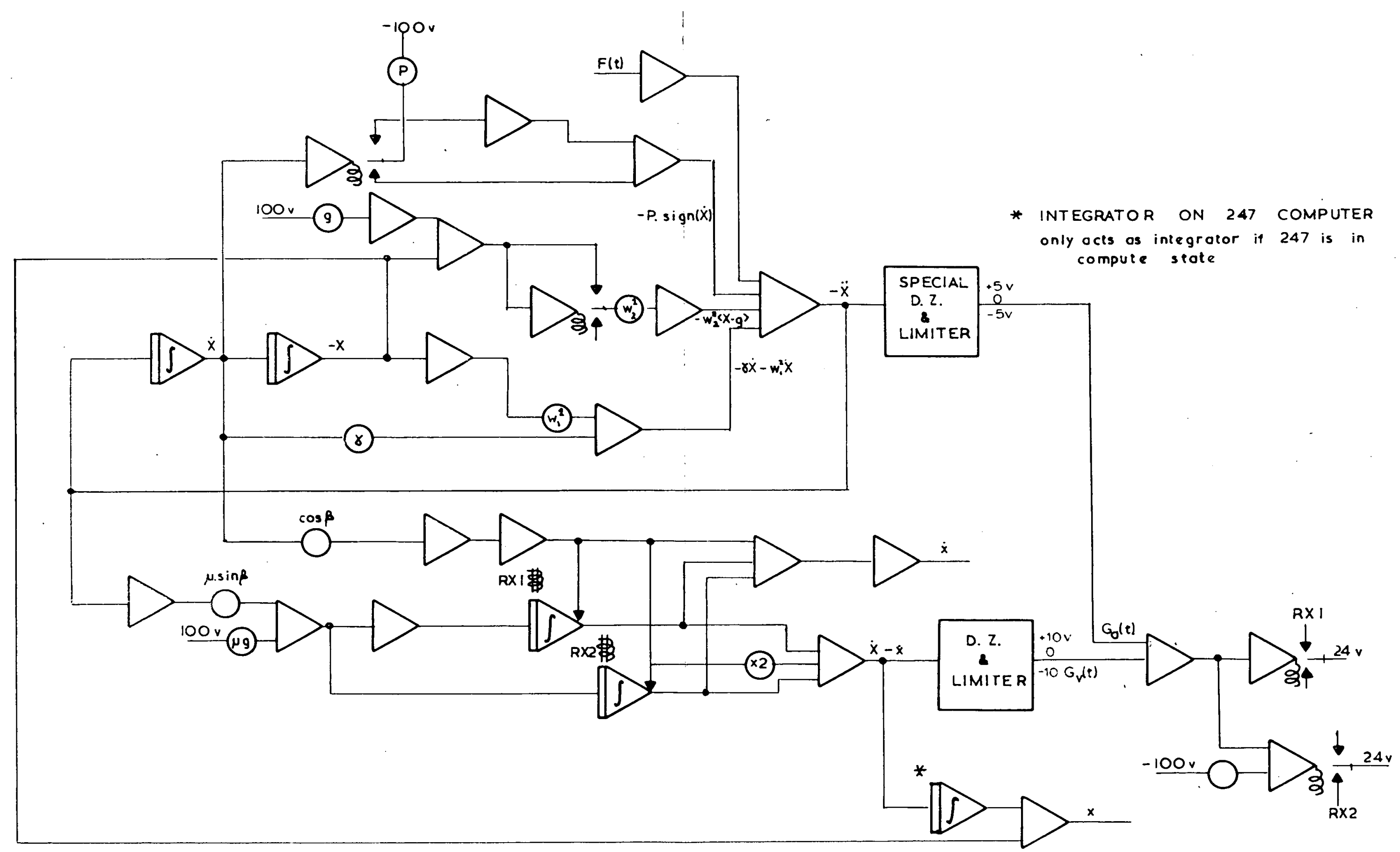

analogue computer circuit for particle motion with table MOtion at angle $B \geqslant 0^{\circ}$ SOME AMPLIFIERS ARE PUT IN CIRCUIT FOR MONITOR PURPOSES 


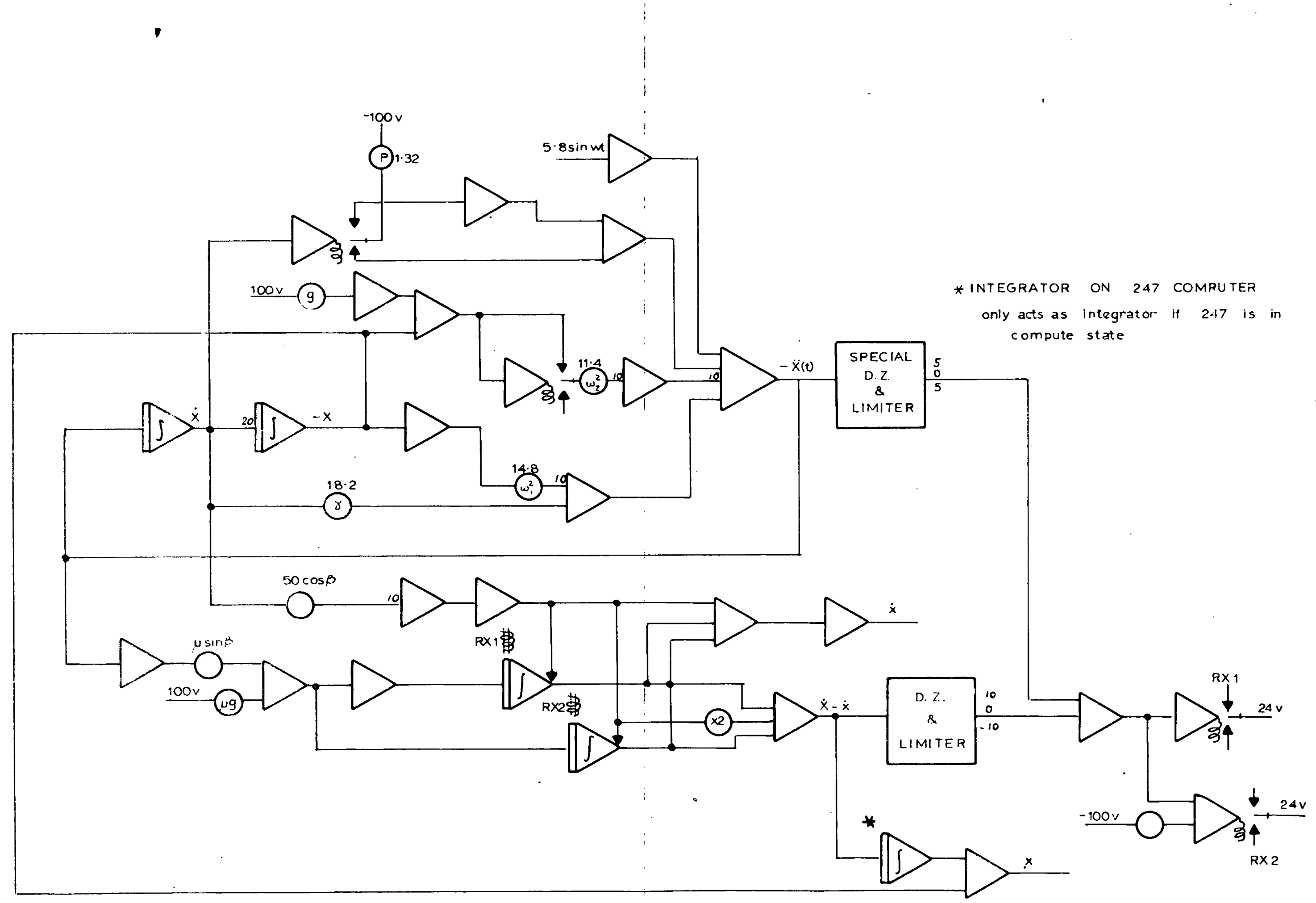

analogue computer circuit for particle motion with table motion at angle $\beta \geq 0^{\circ}$ COEFFICIENTS SET FOR SYSTEM B

FIG. $4: C 4$ 


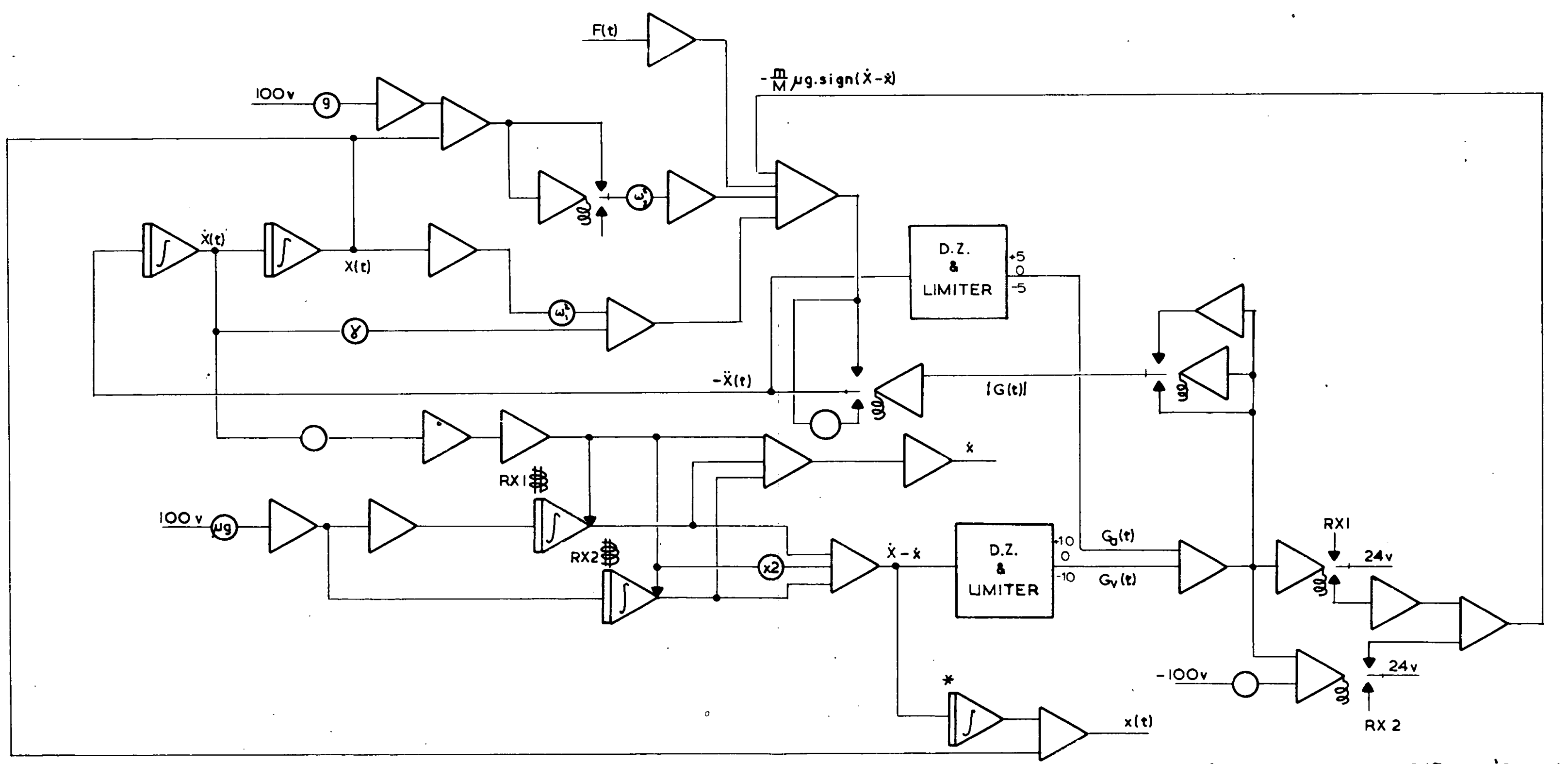




\section{CHAPTER 5}

\section{SOLUTION USING A DIGITAL COMPUTER}

The various techniques for solving non-linear differential equations, and in particular those which have a non-linear restoring force have been set out in section 2. These are (a) exact analytic solutions; (b) approximate analytic solutions (c) analogue computer methods; (d) experiments on a real system and (e) numerical methods. Method (a) is not normally available when investigating non-autonomous systems. The difficulties in applying method (b), the literature on which is alnost entirely devoted to quasi-linear systems have already been described. The previous sections have described the investigation carried out by direct experiment and an analogue computer circuit. This section is devoted to method $(e)$ numerical methods and in particular to the use of a digital computer to obtain a solution.

The advent of high speed digital computers in the last few years has made the solution of differential equations by iterative mothods a practical proposition, though it would appear that the literature on this subject is somewhat lagging behind the practice. While the most widely used iterative procodure, that of Rauscher (29), dates from 1938 its use has been severely limited by the tedious calculations involved, though several researchers ${ }^{(19)}$ have used the method with success and others have developed variations of it. Other methods which have been used are those of Inglis $\left(1_{4}\right)$, and Martinsen ${ }^{(22)}$, the only reference of the latter's use being in (20).

Piece-wise linear systeins have attracted several authors who have looked for simplifications in their solution, and while some have 
achieved greater convenience, others have obtained this only for symmetrical non-linearities. However it was felt that the piecewise linear nature of the problem might offer some simplification.

5.I A quick approximate method to find the waveform of a non-linear single degree of freedom system with an unsymmetric bilinear spring characteristic.

Starting from the equation

$$
\ddot{M} \ddot{X}+C \dot{X}+k(X)=\hat{F} \sin \omega t
$$

with

$$
\begin{aligned}
k(X) & =K_{1} X & & x \leq x_{g} \\
& =K_{1} g+K_{2}\left(x-x_{g}\right) & & x \geqslant x_{g}
\end{aligned}
$$

the motion can be divided into two regions in each of which it is defined by a linear differential equation. These give solutions

$$
x=e^{-n t^{\prime}}\left(A \cos p_{I} t^{\prime}+B \sin p_{1} t^{\prime}\right)+z_{1} \sin \left(\omega t-\alpha_{1}\right) \quad X \leq g
$$

and $x=e^{-n t "}\left(C \cos p_{2} t^{\prime \prime}+D \sin p_{2} t^{\prime \prime}\right)+z_{2} \sin \left(\omega t-x_{2}\right)+g$

$$
\begin{array}{rl}
-\left(\frac{\mathrm{K}_{1}}{\mathrm{~K}_{2}}\right) \mathrm{g} & \mathrm{x} \\
& \geqslant \mathrm{g} \\
\ldots & 5.2
\end{array}
$$

where $A, B, C$ and $D$ are constants determined from the initial conditions at entry to the region of the solution, $n, p, Z$ and are the damping factor, the natural frequency of the system on its springs, and the amplitude and phase angle of steady state forced vibration respectively; t' and t"l are time measured from the moment of entry to a particular region. These equations give exact solution to the motion if $A, B, C$ and $D$ are found such that the motion is periodic and both displacement and velocity curves are continuous. If, in addition to $A, B, C, D, t_{1}$ and $t_{2}$, the instants of ontering and leaving the hard spring region are considered, six unknowns exist. Conditions of continuity of displacement and velocity can be written 
down, but the solution is escessively complicated. The solution obtained by Den Hartog ${ }^{(11)}$ to the case of a symmetrical non-linearity is of little practical use due to its complexity. Various approximations can be made which enable a solution to be found.

If damping is small, so that the exponential terms can be neglected

and

$$
\begin{array}{ll}
X=A \cos p_{1} t^{\prime}+B \sin p_{1} t^{\prime}+Z_{1} \sin \left(\omega t-\alpha_{1}\right) & x \leq g \\
x=C \cos p_{2} t^{\prime \prime}+D \sin p_{2} t^{\prime \prime}+Z_{2} \sin \left(\omega t-\alpha_{2}\right)+g-\left(\frac{K_{1}}{K_{2}}\right) g & x \geqslant g
\end{array}
$$

and

$$
\begin{array}{r}
\alpha_{i}=\tan ^{-1}\left(\frac{2 n \cdot i}{p_{i}^{2}-\omega^{2}}\right) ; \quad z_{i}=\left(\frac{\hat{F}}{K_{i}}\right) / \sqrt{\left(1-\frac{\omega^{2}}{p_{i}^{2}}\right)^{2}+\frac{4 n^{2} \omega^{2}}{p_{i}^{4}}} \\
\ldots 5.3
\end{array}
$$

Since the hard spring is very much more stiff than the soft spring it is reasonable to assume that the region associated with the resonance curve, whose 'back-bone' starts at $p_{1}$, is considerably below the frequency $p_{2}$. This allows the approximations $\alpha_{2}=0$ and $z_{2}=\hat{F} / K_{2}$ which is the static deflection of the hard spring under a force $\widehat{F}$. An estimate may be made of the phase relationship between the force and the motion. If the system is being excited at frequencies such that it is responding in the large amplitudes associated with the non-linear resonance curve below the 'drop-off' frequency, then the response is below the inatural (autoperiodic) frequency, and in the absence of damping the fundamental harmonic content of the motion will be in-phase with the force. This assumption can then be extended to say that if the higher harmonic content is small, the position of maximum displacement of the system on the hard side occurs at the position of maximum force i.e. $\omega t=\pi / 2$. Returning to the second of equations (5.3) and putting $X=g$ at $t "=0$ gives $C=\left(\frac{K_{1}}{K_{2}}\right) g-z_{2} \sin \omega t$ which is small 
since $K_{2}$ is large compared with $K_{1}$ and $z_{2}$ is small. Thus $D_{2} \operatorname{sinp} p_{2}$ " is the dominant term in the second equation. To a first approximation the region in which $X \geq g$ will last for a period of $\frac{1}{2}$ cycle of motion at the natural frequency of the system $p_{2}$. inis contact with the hard spring occurs at $\omega_{t}=\pi / 2-\frac{\pi}{2} \frac{\omega}{p_{2}}$ and lasts until $X=g$ which is given by $t=\frac{\pi}{2}+\frac{\pi}{2} \frac{\omega}{p_{2}}$. This establishes two blues of $t$ for which $X=g$ for each region. The values of $t$ used in the first approximation are

$\begin{array}{lll}\text { At entry to the hard spring } & p_{2} t^{\prime \prime}=0 & t=\frac{\pi}{2}-\frac{\pi}{2} \frac{\omega}{p_{2}} \quad \text { (i) } \\ \text { On leaving the hard spring } & p_{2} t^{\prime \prime}=\pi & t=\frac{\pi}{2}+\frac{\pi}{2} \frac{\omega}{p_{2}} \quad \text { (ii) } \\ \text { At entry to the soft spring } & p_{1} t^{\prime}=0 & t=\frac{\pi}{2}+\frac{\pi}{2} \frac{\omega}{p_{2}} \quad \text { (iii) } \\ \text { On leaving the soft spring } & p_{1} t^{\prime}=\left(2 \pi-\frac{\pi \omega}{p_{2}}\right) \frac{p_{I}}{t=2 \pi+\frac{\pi}{2}-\frac{\pi}{2} \frac{\omega}{p_{2}} \quad \text { (iv) }} \\ & & \ldots 5.4\end{array}$

The calculation is carried out using equations (i.), (iii) and (iv) to find $C, A$ and $B$. Since $C$ is small the motion in the region $X \geqslant g$ is almost symmetrical, so the velocities at entry to the region and on leaving the region should be approximately equal in magnitude though of opposite sign. Knowing A and B these velocities can be calculated, but as neither the above condition of symmetry, nor even the condition of continuity of velocity have been introduced there is no guarantee that the two values of velocity will be even approximately equal, though experience has shown that they often are. From the average of these two velocities an estimate of $D$ can be found. If better accuracy is desired, this value of $D$ can be used to calculate the exact interval spent when $X \geqslant g$, considering $C$ to be finite. As the motion will not be exactly in-phase, but almost certainly lagging behind the 


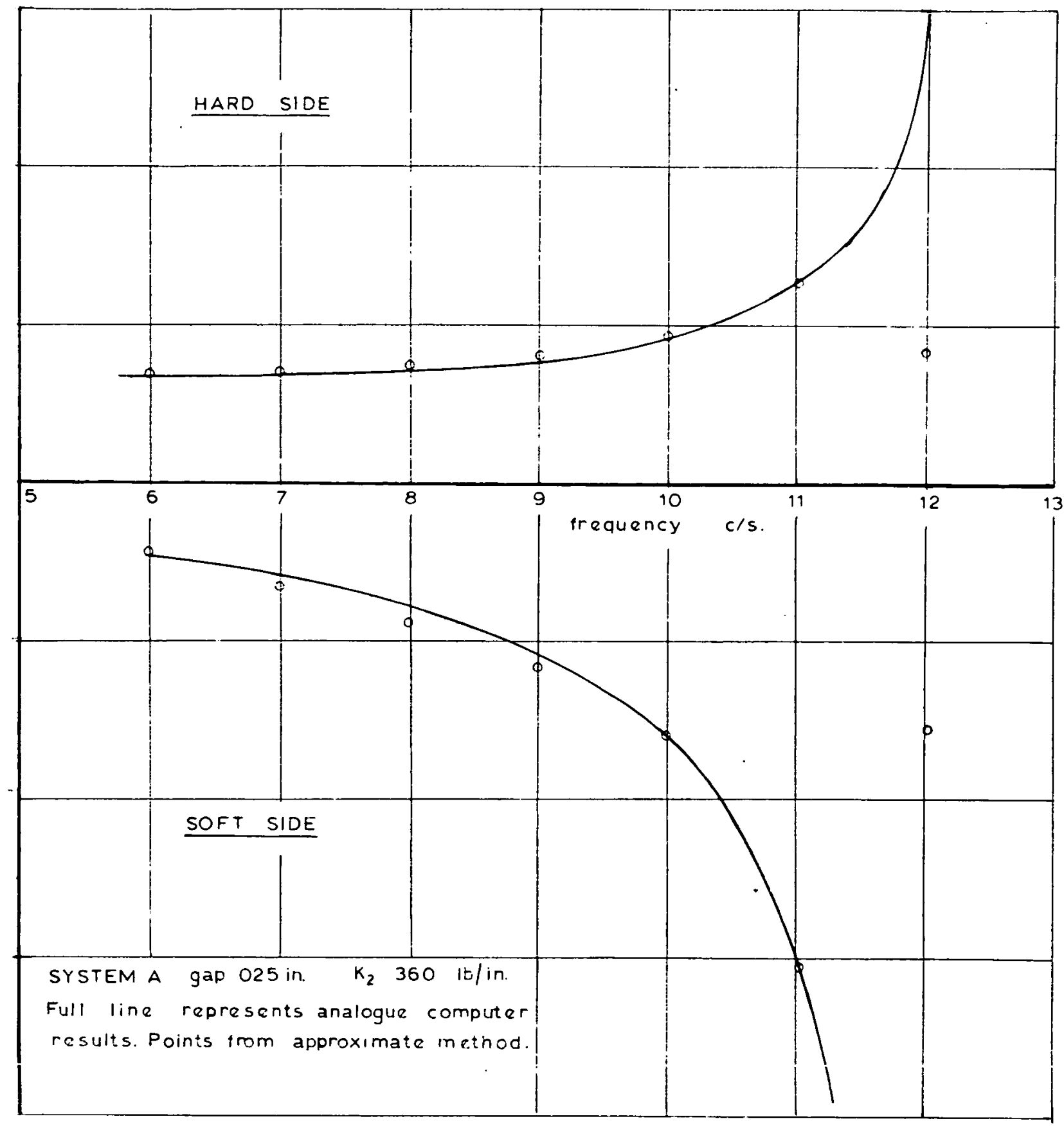

COMPARISON OF MAXIMUM AMPLITUDES FROM ANALOGUE COMPUTER \& APPROXIMATE METHOD.

FIG. $5: 1$ 
force by a small phase angle, the instant of entry is retained as $t=\frac{\pi}{2}-\frac{\pi}{2} \frac{\omega}{p_{2}}$, and any increase in the period within the region added onto the values previously specified in (ii) equation (5.4). Repeating the calculation will in general give good enough agreement, but if not, the period in which $X \geqslant g$ is not changed, but a further phase shift is introduced by making $\omega t=\omega t+\epsilon$ in equations $(5.4)$, when one or two calculations with different $\epsilon$ are usually enough to give agreement. On average such a calculation using slide rule and tables of trigonometrical functions would take $20-25$ minutes.

Once the solutions have been found it is noted that they are almost exact solutions, the only error, other than the degree of approximation used, being the absence of the exponential damping term. Two curves are shown in Fig. 5.1 giving an idea of the accuracy which can be achieved. The trace for $11 \mathrm{c} / \mathrm{s}$ is a first approximation. This method has advantages over the more usual numerical methods in both speed and accuracy though its mathematical basis is hardly elegant.

The method is not unfortunately, suitable for subharmonic motion when the table response is periodic in two or more cycles of force. Nor does it hold when the mass enters the region $X \geqslant g$ more than once per cycle. If, however, a solution has been assumed to have one such region per cycle when in fact it has two, this will emerge from the solution, in that if the conditions are satisfied as described above, the resulting waveform will be found to have a second region $X \geqslant g$ which, of course, has not been considered in the solution. These restrictions on the use of the method are no more stringent than on any other numerical method, all of which have been developed for harmonic solutions whose motion is, in the first approximation, that 


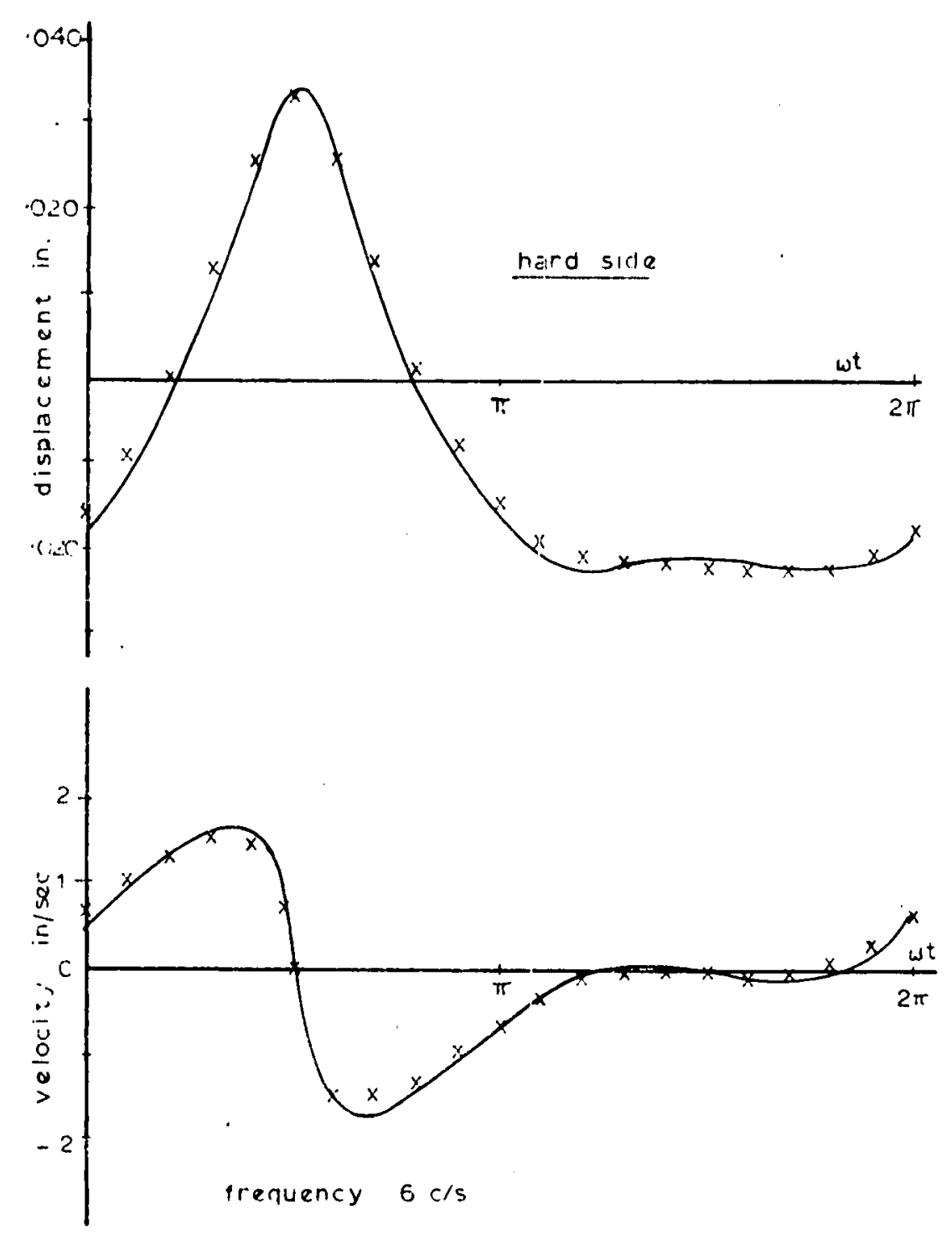

tull line shows results by aporoximate method points are ligieal crmputer srlution COMPARISON OF WAVEFORMS OBTAINED DIGITAL COMPUTER.-(SYSTEM A gap.O25 in. $K_{2} 360 \mathrm{lb} / \mathrm{in}$.)

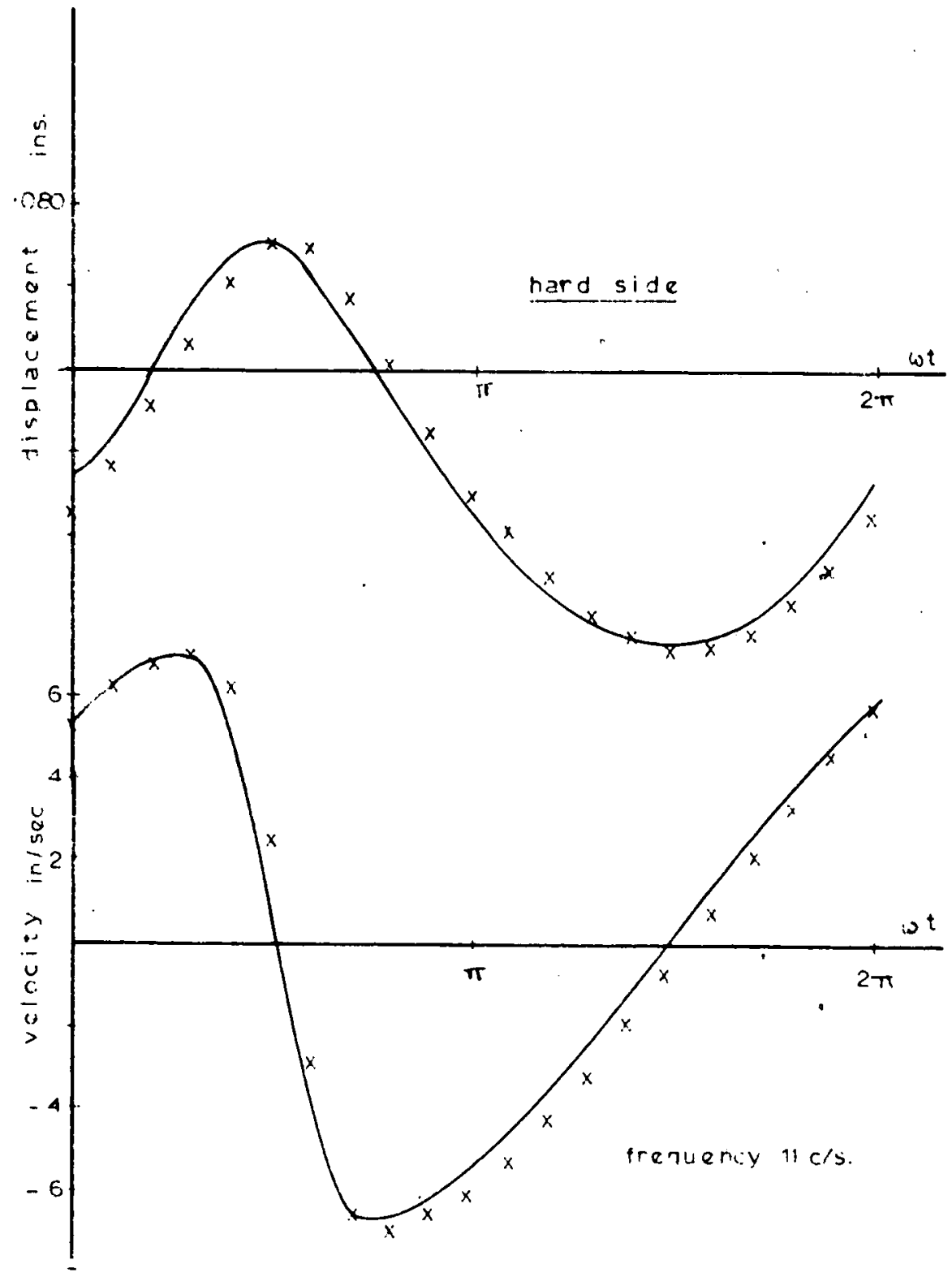

BY APPROXIMATE METHOD AND BY 
of the system in free vibration. If more than one region $X \geqslant g$ occurs then the deviation from this waveform will be very marked.

A further possibility of the method not fully investigated, is that estimates can be made of the table motion in the unstable region of the resonance curve. As it was previously assumed that the motion was in-phase with the force, so it can be assumed that below the 'backbone' of the non-linear resonance curve the motion is completely out of phase, and by reasoning similar to that applied above equations of the waveform can be obtained. This solution, when found, completely satisfies the equations, there being no indication as to its lack of stability. This type of solution cannot be obtained by experiment or by analogue computation, and only a numerical method carefully designed would obtain this solution instoad of diverging to one of the two stable solutions.

Graph 5.2 shows the response for one system investigated by this method and allows comparison with exact analogue gomputer and digital computer results. Travel rates for the horizontally vibrating table with a particle slider were also obtained and are shown in graph 6.7 This approximate method, proved a valuable means of achieving more rapid convergence to steady state on a digital computer. 5.2

The comparative complexity and amount of calculation required to obtain accurate solutions by numerical methods indicated that if many results were required a digital computer would have to be used. As mentioned, the literature on the use of digital computers for solving dynamic systems does not seem to have progressed as far as might be expected. The experimental work indicated that whatever 
method was used to solve the equations would require the capacity to produce sub-harmonic as well as harmonic solutions, and also be capable, without using excessive machine time, of calculating solutions in which contact was made with the hard spring more than once in each cycle of force. Looking further ahead beyond the case of the single degree of freedom system representing a. vibrating conveyor with a particle being transported where one equation describes the table motion over a cycle, the program evolved would have to be capable of finding the motion and travel rates of a mass being transported which is comparable to that of the table. In this case three equations may be needed to describe the conveyor motion over one cycle, the regions in which each equation applied being a function of the table motion.

The limitations of numerical methods have been briefly mentioned. However, considering only the single degree of freedom system, it would be possible to write a program based on the method of Rauscher to find a harmonic solution at a given frequency, though as previously mentioned, if the waveform is distorted by the presence of higher harmonics such that it is very different from the free vibration waveform, a considerable number of iterations may be required to give the solution. There would be no indication as to whether the solution was stable or unstable, or whether a sub-harmonic solution was possible. When more than one equation holds during a cycle, the iteration cannot be used, since the correction in conditions at the start of the cycle to cause convergence to a periodic solution is not known.

The method finally adopted was based on the bi-linear spring characteristic which allows the table motion to be represented by one or more pairs of linear differential equations whose solution is known. 
The digital computers to which access was available during the . course of the investigation were an 'Atlas' computer at Manchester University, and English Electric Leo Marconi KDF 9 computers at Newcastle, Glasgow and latterly, at the regional computing gentre in Edinburgh. The machines were programmed in 'Atlas' autocode for an Edinburgh University compiler, the input being on 7 hole tape.

The investigation of the conveying speeds of vibratory conveyors by the digital machine developed along similar lines to the analogue investigation. The first program produced solved the single degree of freedom system with a particle being conveyed, this being exactly the case of the 'small' circuit of the 'Minispace'. The second programme gave the slider motion as well as the table motion and allowed the case of a mass being transported which was comparable to the table mass. Finally an 'omnibus' programme was evolved which embraced both previous cases, as well as solving the problem for inclination of the plane of vibration at $\beta^{\circ}$ to the horizontal both with a negligible mass and a finite mass. A further modification to this programne allowed it to be used for the case of a particle on a vibrating table which has a maximum vertical acceleration greater than that of gravity.

Before describing these programmes it should be stated that it must not be assumed that the final 'omnibus' programme necessarily supersedes the previous ones. Each additional complexity introduced requires more machine time and speed of computation is slowed down. In general the most simple programme which will solve the particular problem should be used, rather than a more complex program used to much less than its capacity. 


\subsection{The basic computer programme}

equation of motion

$$
\ddot{M X}+C \dot{X}+k(X)=F \sin \omega t
$$

with

$$
\begin{array}{rlrl}
k(x) & =K_{1} X & & x \leq x_{g} \\
& =K_{1} g+K_{2}(x-g) & x \geqslant g
\end{array}
$$

can be solved by writing the two solutions for each region and establishing continuity conditions of velocity and displacement at each boundary $X=g$. The solutions are

$$
\begin{array}{lr}
X=e^{-n t^{\prime}}\left(A \cos p_{I} t^{\prime}+B \sin p_{1} t^{\prime}\right)+z_{1} \sin \left(f t-x_{1}\right) & X \leq g \\
x=e^{-n t^{\prime \prime}}\left(C \cos p_{2} t^{\prime \prime}+D \sin p_{2} t^{\prime \prime}\right)+z_{2} \sin \left(f t-\alpha_{2}\right)+g-\left(\frac{K_{I}}{K_{2}}\right) g \quad & X \geqslant g \\
& \ldots 5.6
\end{array}
$$

The equations (5.5) and (5.6) are identical to (5.1) and (5.2). As discussed in section 5.1 they are solved by establishing conditions of continuity of velocity and displacement at the boundaries of the regions. If this continuity is used as a condition in generating the new constants of the region being entered, then the resulting solution is an exact one both in the transient state and in the periodic steady state. A periodic solution is achieved if both displacenent and velocity have the same values at two instants of time $\tau$ apart, where $\tau$ is a multiple, or subrultiple, of the period of the forcing function. The calculation can be carried out from any initial condition, but the number of cycles required to obtain a periodic solution will depend on the accuracy of the initial conditions used. Unlike an analogue computer, there is no facility on a digital programme by which a solution can be disturbed from steady state to see if it will return to its previous solution or whether in fact another stable 
solution, possibly less dominant, coexists. This was investigated by re-running the program with different initial conditions.

The general arrangement of the programme can be seen in the accompanying block diagram.

Any programme is written according to fairly rigid formulae laid down by the compiler. After a formal job heading all variables to be used were declared. From the numerical data read in - spring characteristic table mass, frequency of excitation, amplitude of force and damping - the steady state forced vibration amplitudes and phase angles were calculated $\left(z_{1}, z_{2}, x_{1}, \alpha_{2}\right)$. The initial conditions were then set as required. In general the approximation developed in section 5.1, which was found to be particularly powerful in aiding convergence, was used. There-after the calculation proceeded by calculating the displacement $X(t)$ at discrete intervals of $1 / 100$ of a cycle of force for a maximum of 60 cycles. It should be noted that while in the analogue circuit the highest order differential term is integrated to obtain the displacement, in the digital programme the displacement was known, velocity and acceleration being found differentiating the formula for displacement. No machine integration or differentiation is required when exact solutions are known. At each value of displacement calculated a check was carried out to see if the displacement waveform had passed through the boundary of the hard spring. If this occurred the exact time $t_{0}$ at which $X\left(t_{0}\right)=g$ was found by linear interpolation. A more accurate method using Newton-Rapheson iteration was tried but produced no significant change in solutions, and required more machine time. Knowing to the velocity $\dot{X}\left(t_{0}\right)$ was calculated and from it the constants of the 


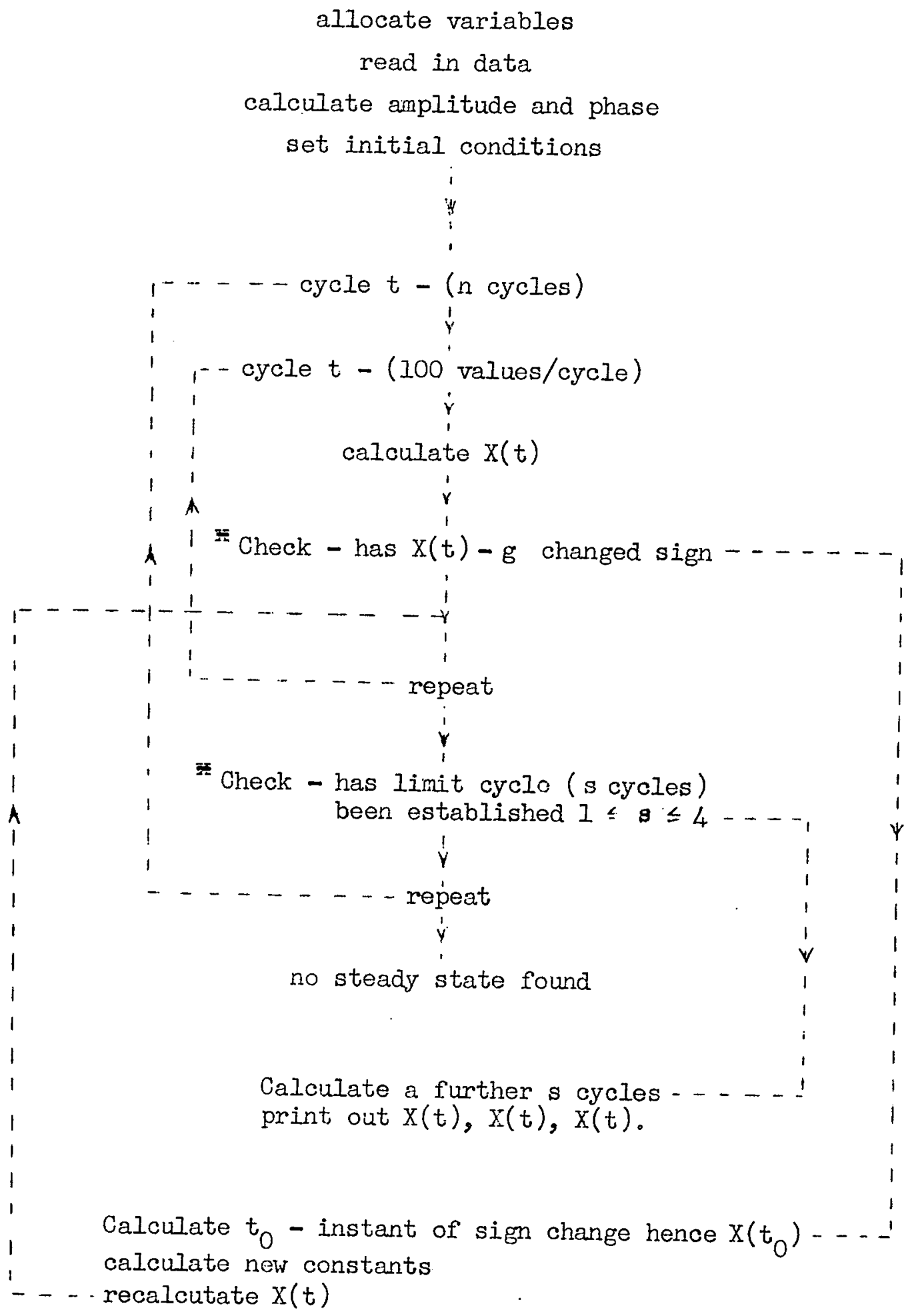

Simplified block diagram of computer circuit 
new region being entered. After 25 cycles it was assumed that there was a reasonable chance that steady state might have been obteined. Hence the values of displacement and velocity at the end of the 26th and each successive cycle were compared with those at the end of the preceeding three cycles. This would establish cycle of s cycles $(1 \leq s \leq 4)$ was established the computation was continued for a further $s$ cycles, with print-out of displacement and velocity.

In this way waveforms were obtained for various spring configuration for a range of frequencies 5-15 cycles/sec. or as much of that region as was associated with the resonance curve. The waveforms were plotted on graph paper and the slider velocity obtained by planimeter exactly as described in the section on the 'Minispace' analogue computer. As a means of obtaining a digital solution to the differential equation incorporating a bilinear spring force, the program proved most successful the solutions being subharmonic in those regions which had previously been found by experiment and analogue computer methods. It did, however, suffer from all the limitations and disadvantages of the small analogue computer, and it was obvious that a nore sophisticated programme was required.

5.4 A computer programme for the solution of the case of horizontal table motion with a mass being conveyed which may be comparable to the table mass.

As in the analogue computer it was more convenient to generate. the velocity vaveform of the material being transported, and find the travel rate within the machine.

The equations of motion are

a) no slipping $(M+m) \ddot{X}+C \dot{X}+k(X)=\hat{F} \sin \omega t$

$$
\dot{\mathrm{x}}=\dot{\mathrm{x}}
$$


b) slipping $\quad \ddot{M}+\dot{C X}+k(X)+\mu m g \operatorname{sign}(\dot{X}-\dot{X})=\hat{F} \sin \omega_{t}$

$$
\ddot{x}=\mu \operatorname{sign}(\dot{X}-\dot{x})
$$

... 5.7

where slip is initiated by

and continues until

$$
\begin{aligned}
& \ddot{x}= \pm \mu g \\
& \dot{x}=\dot{x}
\end{aligned}
$$

These equations were broken down into six pairs depending on (a) whether the stable was in the 'soft' spring region or the 'hard' spring region, and (b) on whether the slider was not slipping, or slipping in a positive or negative direction relative to the table for either of regions (a). It was found most convenient to have a convenient reference of which region the motion was in at any time. This was done by using the integer $\underline{v}$ to refer to the six regions. Region $1 \quad(\mathrm{v}=1) ; \mathrm{X} \leq \mathrm{X}_{\mathrm{g}}$; no relative slipping

$$
\begin{aligned}
(M+m) \ddot{X}+C \dot{X}+W_{I} X & =\hat{F} \sin \omega t \\
x & =X
\end{aligned}
$$

Region $2(v=2) ; \quad x \leq x_{g} ; \dot{X}>\dot{x} ; \quad$ slipping negatively in soft region

$$
\begin{aligned}
\ddot{M}+C \dot{X}+K_{I} X+\mu m g & =\hat{F} \sin \omega t \\
\ddot{x} & =\mu g
\end{aligned}
$$

Region $3 \quad(v=3) ; \quad x \leq x_{g} ; \dot{X}<\dot{x} ;$ slipping positively in soft region

$$
\begin{aligned}
M \ddot{X}+C \dot{X}+K_{I} X-\mu m g & =\hat{F} \sin \omega t \\
\ddot{x} & =-\mu g
\end{aligned}
$$

Region $4 \quad(v=4) ; \quad X \geq X_{g} ;$ no relative slipping

$$
\begin{aligned}
(M+m) \ddot{X}+C \dot{X}+K_{1} X_{g}+K_{2}\left(X-X_{g}\right) & =\hat{F} \sin (v t \\
x & =X
\end{aligned}
$$

Region $5(\mathrm{v}=5) ; \quad \mathrm{X} \geq \mathrm{X}_{\mathrm{g}} ; \dot{\mathrm{X}}>\dot{\mathrm{x}} ;$ slipping negatively in hard region

$$
\begin{aligned}
\ddot{M X}+C \dot{X}+K_{1} X_{g}+K_{2}\left(X-X_{g}\right)+\mu m g & =\hat{F}_{\sin \omega t} \\
\ddot{x} & =\mu g
\end{aligned}
$$


Region $6(v=6) ; \quad x \geq X_{g} ; \dot{x}<\dot{x} ; \quad$ slipping positively in hard region

$$
\begin{aligned}
\ddot{M X}+C \dot{X}+K_{1} X_{g}+K_{2}\left(X-X_{g}\right)-\mu m g & =\hat{F} \sin \omega t \\
\ddot{X} & =-\mu g \quad \ldots 5.8
\end{aligned}
$$

where $\mathrm{X}_{0}$ is velocity at entry to a particular region.

The conditions of application of any region are tabulated below.

\begin{tabular}{|c|c|c|}
\hline Region & Conditions of entry & Conditions for leaving \\
\hline 1 & $\mathrm{X} \leq \mathrm{X}_{\mathrm{g}} ; \dot{\mathrm{x}}=\dot{\mathrm{X}} ; \quad|\ddot{\mathrm{x}}|<\mu \mathrm{g}$ & $\mathrm{x}>\mathrm{x}_{\mathrm{g}} \stackrel{\circ}{\circ}|\ddot{x}|>\mu \mathrm{g}$ \\
\hline 2 & $\begin{array}{l}x \leq X_{g} ; \dot{x}=\dot{X} ; \ddot{x}>\mu g \\
\text { or } x \leq x_{g} ; \dot{x}>\dot{x}\end{array}$ & $x>x_{g}$ or $\quad \dot{x}=\dot{x}$ \\
\hline 3 & $\begin{array}{l}x \leq x_{g} ; \quad x=x ; \quad \ddot{x}<-\mu g \\
\text { or } x \leqslant x_{g} ; \quad \dot{x}<\dot{x}\end{array}$ & $x>x_{g}$ or $\quad \dot{x}=\dot{x}$ \\
\hline 4 & $\mathrm{x} \geqslant \mathrm{x}_{\mathrm{g}} ; \quad \dot{\mathrm{x}}=\dot{\mathrm{x}} ; \quad|\ddot{\mathrm{x}}|<\mu \mathrm{g}$ & $x<x_{g}$ or $|\ddot{x}|>\mu g$ \\
\hline 5 & $\begin{array}{l}x \geqslant x_{g} ; \quad \dot{x}=\dot{x} ; \quad \ddot{x}>\mu g \\
\text { or } x \geqslant x_{g} ; \dot{x}>\dot{x}\end{array}$ & $x<x_{g}$ or $\dot{x}=\dot{X}$ \\
\hline 6 & $\begin{array}{l}X \geqslant X_{g} ; \quad \dot{x}=\dot{X} ; \quad \ddot{x}<-\mu g \\
\text { or } X \supseteq x_{g} ; \quad \dot{X}<\dot{x}\end{array}$ & $x<x_{g}$ 으 $\dot{x}=\dot{x}$ \\
\hline
\end{tabular}

\section{TABLE 5.1}

3 on entry from region 1

on entry from region 4

The use of the above table will probably be made more clear by considering an example. If the motion is in region 1 and the displacement $X(t)$ exceeds $X_{g}$ without any other change the system enters 
region 4. If $\ddot{X}(t)$ the acceleration becomes less than $-\mu g$ region 6 will be entered. If $X(t)$ becomes less than $X_{g}$ without any other change the system will enter region 3. There is a proviso that changes between regions 2 and 3 may only occur via region 1 , and similarly changes between regions 5 and 6 may only occur via region 4, though the changes may occur at a particular instant of time. That is to say instantaneous reversal of the direction of relative slip occurs by considering that there is no relative slip instantaneously, but that if the conditions for slip to start are fulfilled then the new region is entered immediately. Returning to equations 5.8 the solution to the table motion can be written as

$$
X(t)=e^{-n(v) t^{\prime}}\left\{A(v) \cos p(v) t^{\prime}+B(v) \sin p(v) t^{\prime}\right\}+Z(v) \sin (\omega t-\alpha(v)+S(v)
$$

when $t^{\prime}$ is time measured from the instant of entry to a region. for all values of $\mathrm{v}$, and

$$
\begin{aligned}
\dot{x}(t) & =\dot{x}(t) & v & =1 \text { or } 4 \\
& =\dot{x}\left(t_{0}\right)+u(v)\left(t-t_{0}\right) & v & =2,2,5,6
\end{aligned}
$$

where $u(v)$ means the value of $u$ in region $v$.

Damping would normally be measured by decrement or band-width techniques from the system when the table has no added mass $\left(\frac{C}{M}=2 n\right)$ aria $x<\pi_{g}$ Then

$n(1)=n\left\{\frac{M}{M+m}\right\} ; n(2)=n ; n(3)=n ; n(4)=n\left\{\frac{M}{M+m}\right\} ; n(5)=n ; n(6)=n$

$p(I)=\sqrt{K_{I} /(M+m)} ; p(2)=\sqrt{K_{I} / M} ; p(3)=\sqrt{K_{I} / M} ; p(4)=\sqrt{K_{2} /(M+m)} ;$

$\mathrm{p}(5)=\sqrt{\mathrm{K}_{2} / \mathrm{M}} ; \mathrm{p}(6)=\sqrt{\frac{\mathrm{K}_{2}}{1}}$ 
then

$$
\begin{aligned}
Z(v)=\frac{\hat{F}}{K(v)} / \sqrt{\left(1-\frac{\omega^{2}}{p^{2}(v)}\right)^{2}+\frac{4 \cdot n^{2}(v) \cdot \omega^{2}}{p^{4}(v)}} ; \alpha(v)=\tan ^{-1}\left\{\frac{2 n(v) \cdot \omega}{p^{2}(v)-\omega^{2}}\right\} \\
K(v)=K_{1} \quad v=1,2,3 \\
=K_{2} \quad v=4,5,6
\end{aligned}
$$

$S(v)$ embraces all the constant terms in the expression

$$
\begin{aligned}
& S(1)=0 ; \quad S(2)=-\mu \mathrm{mg} / \mathrm{K}_{1} ; \quad S(3)=\mu \mathrm{mg} / \mathrm{K}_{1} \\
& S(4)=X_{g}-\left(\frac{K_{1}}{K_{2}}\right) \cdot x_{g} ; S(5)=x_{g}-\left(K_{I} / K_{2}\right) \cdot x_{g}-\mu m g / K_{2} ; \\
& S(6)=X_{g}-\left(\frac{K_{l}}{K_{2}}\right) X_{g}+\mu m g / K_{2}
\end{aligned}
$$

$A(v)$ and $B(v)$ do not have quite the same meaning as the other functions. While $A(v)$ and $B(v)$ are constants over a region, their values are calculated at entry to that region. It is often convenient, however, to retain the constants of the previous region after the new constants have been calculated.

For the slider

$$
u(2)=\mu g ; u(3)=-\mu g ; u(5)=\mu g ; u(6)=-\mu g \quad \ldots 5.11
$$

The advantages of this method of notation can be seen with reference to programme 1 . If the table is vibrating in region $I, v=I$ and the displacement becomes greater than $X_{g}$, then the new region is $(\mathrm{v}+3 \dot{X}=4)$ and if the new $A(v)$ and $B(v)$ are calculated, then the first of equations (5.9) represents the motion. If then $\ddot{X}<-\mu g$ then $v=v+2$ $(=6)$. If $x(t)<x_{g}$ with no other change $v=v-3=3$. These values of $\mathrm{v}$ can be tabulated. 


\section{TABIE 5.2}

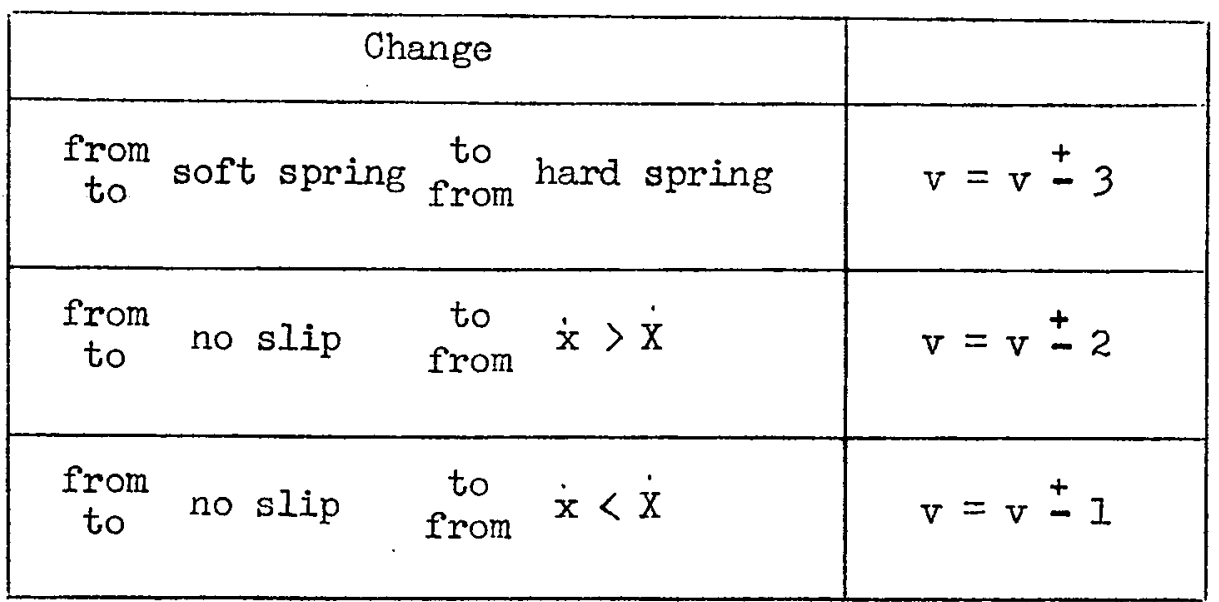

The computation was carried out as before with values of displacement velocity and acceleration being calculated 100 times each cycle of force. While in the previous programme only displacement was calculated, the velocity only boing required at entry to a new region, when the slider motion is wanted, acceleration and velocity of the table must be calculated at each value some rather more complicated switching conditions were required, particularly as the condition $\mathrm{X}=\mathrm{X}_{\mathrm{g}}$ often coincided with a change from backward to forward relative slip. In this more complicated programme no check was made for limit cycles. The computation was carried out for 60 cycles of force, the last 4 cycles being used to give print out of the various velocities and displacements. Also during these last 4 cycles the travel rate in inches/cycle was obtained by integrating the relative velocity using Simpson's Rule.

Similarly $X(t)$ the slider displacement can be obtained from

$$
x(t)=x(t-\tau)+\frac{1}{2}\{\dot{x}(t)-\dot{x}(t)+\dot{x}(t-\tau)-\dot{x}(t-\tau)\} \tau+x(t)
$$

where $\tau$ is the interval between successive calculations $(1 / 100$ of the period of the forcing function). 
It is not likely that in investigations of resonant systems the power requirement will be important. However this was easy to obtain and several results were taken. Since both force (Fsin $t$ ) and table velocity were known at 100 intervals per cycle of force, the work done by the force

$$
W \cdot D=\int F d x=\int_{0}^{T} F \dot{X} d t
$$

could be obtained using Simpson's Rule.

On K.D.F 9 the computing rate for this programme is about 15 cases of different frequency and/or spring characteristic per 30 minutes computing time. 5.5 Programme extended to embrace the case of inclination at $\beta^{\circ}$ to the

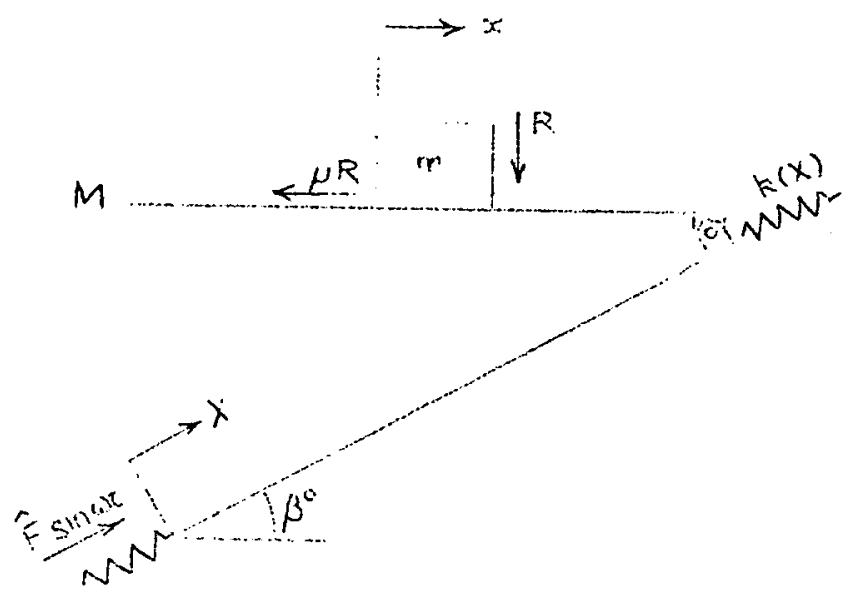

The equations of motion for this case have been developed in section 2. They are for the table

a) no slip

$$
(M+m))^{\prime \prime}+C \dot{X}+k(X)=\hat{F} \sin \omega t-m g \sin \beta
$$

b) slip

$$
\begin{aligned}
\left(M+m \sin ^{2} \beta+\mu m \sin \beta \cos \beta \operatorname{sign}(\dot{X} \cos \beta-\dot{x})\right) X+c \dot{X}+k(X) \\
=\hat{F} \sin \omega t-m g \sin \beta-\mu m g \cos \beta \operatorname{sign}(\dot{X} \cos \beta-\dot{X})
\end{aligned}
$$


and for the slider

a) no slip $\dot{x}=\dot{x}$

b) $\sin \quad \ddot{x}=\mu(g+\ddot{x} \sin \beta) \operatorname{sign}(\dot{x}-\dot{x} \cos \beta) \quad \ldots 5.12$

As before the motion of table and slider can be defined by 6 pairs of differential equations such of which has a known solution. The general solution of table motion can again be written in the form

$$
X(t)=e^{-n(v) t^{\prime}}\left\{A(v) \cos p(v) t^{\prime}+B(v) \sin p(v) t^{\prime}\right\}+Z(v) \sin (\omega t-\alpha(v)+s(v)
$$

for all values of $v$, and for the slider

$$
\begin{aligned}
& \dot{x}(t)=\dot{x}(t) \quad v=1 \text { or } 4 \\
&=\dot{x}\left(t_{0}\right)+u(v) \int_{t_{c}}^{t}(g+\ddot{x}(t) \sin \beta) d t \quad v=2,3,5,6 \\
& \ldots .5 .13
\end{aligned}
$$

where $u(v)$ is the value of $u$ in region $v$.

Experiments with the programme representing the horizontal case indicated that with the light damping involved no noticeable error resulted from the assumption that $n(v)$ was a constant for all regions. This small simplification was used in the programme. It was mainly a matter of convenience. No difficulty would be experienced in inserting the correct values.

The values of the other coefficients were found as before.

$$
\begin{aligned}
& p(I)=\sqrt{K_{I} /(M+m)} \\
& p(2)=\sqrt{K_{I} /\left(M+m \sin ^{2} \beta+\mu m \sin \beta \cos \beta\right)} \\
& p(3)=\sqrt{K_{I} /\left(M+m \sin ^{2} \beta-\mu m \sin \beta \cos \beta\right)} \\
& p(4)=\sqrt{K_{2 /(M+m)}} \\
& p(5)=\sqrt{K_{2} /\left(M+m \sin ^{2} \beta+\mu m \sin \beta \cos \beta\right)}
\end{aligned}
$$


$p(6)=\sqrt{K_{2 /\left(M+m i^{2} \beta-\mu m \sin \beta \cos \beta\right)}}$

Then

$$
\begin{aligned}
& Z(v)=\frac{\hat{F}}{K(v)} / \sqrt{\left(1-\frac{\omega^{2}}{p^{2}(v)} \cdot\right)^{2}+\frac{4 n^{2} \omega^{2}}{p^{4}(v)}} ; \alpha(v)=\tan ^{-1}\left\{\frac{2 n \omega}{p^{2}(v)-\omega^{2}}\right\} \\
& S(I)=-\frac{m g \sin \beta}{\mathrm{K}_{1}} ; S(2)=-\frac{\mu m g \cos \beta}{\mathrm{K}_{1}}-\frac{m g \sin \beta}{\mathrm{K}_{1}} \\
& S(3)=-\frac{m g \sin \beta}{K_{I}}+\frac{\mu m g \cos \beta}{K_{l}} ; S(4)=x_{g}-\frac{K_{I} / K_{2}}{} \cdot x_{g}-\frac{m g \sin \beta}{K_{2}} \\
& S(5)=x_{g}-\frac{K_{1} X_{g}}{K_{2}}-\frac{m g \sin \beta}{K_{2}}-\frac{m g \cos \beta}{K_{2}} ; S(6)=X_{g}-\frac{K_{1} X_{g}}{K_{2}}-\frac{m g \sin \beta}{K_{2}} \\
& +\frac{m g \cos \beta}{\mathrm{K}_{2}}
\end{aligned}
$$

For the slider

$$
u(2)=\mu ; u(3)=-\mu ; u(5)=\mu ; u(6)=-\mu \quad \ldots 5.14
$$

However it will be noticed that the slider velocity $x(t)$ now involves an integration containing $X(t)$, the table acceleration, which is not known as an analytic expression, and so a numerical integration had to be carried out. The method adopted was to use the simplest approximation possible - that the acceleration $X(t)$ over an interval $\left({ }^{1} / 100\right.$ of the period of the forcing function) was the value at the end of the interval. With the small values of $\tau$ involved it was not felt that any appreciable error would accrue. A rather better approximation would be to obtain an average value of $X(t)$ by assuming the acceleration to be a linear function over the interval. The table velocity was then caldulated as

$$
x(t)=x(t-\tau) \pm \mu(g+\ddot{x}(t) \cdot \sin \beta) \tau
$$

The conditions for entering or leaving a region can be written as in table 5.1. As before $\underline{v}$ was used as a means of identifying the 
region of motion.

TABIE 5.3

\begin{tabular}{|c|c|c|}
\hline Region & Conditions at Entry & Conditions for leaving \\
\hline 1 & $X \leq X{ }_{g} ; \dot{x}=\dot{X} \cos \beta ;|\ddot{X} \cos \beta|<\mu(g+\ddot{X} \sin \beta) \mid$ & $X>X_{g}$ or $|\ddot{X} \cos \beta|>\mu(g+\ddot{X} \sin \beta) \mid$ \\
\hline 2 & $\begin{array}{l}X \leq X{ }_{g} ; \dot{x}=\dot{X} \cos \beta ; \ddot{X} \cos \beta>\mu(g+X \sin \beta)^{z x} \\
\text { or } X \leq X{ }_{g} ; \dot{x}<\dot{X} \cos \beta\end{array}$ & $X>X_{g}$ or $\dot{x}=\dot{x} \cos \beta$ \\
\hline 3 & $\begin{array}{l}X \leq X_{g} ; \dot{X}=\dot{X} \cos \beta ; \ddot{X} \cos \beta<-\beta\left(g+\ddot{X}_{\sin f}\right)^{3} \\
\text { or } X \leq X_{g} ; \dot{X}>\dot{X} \cos \beta\end{array}$ & $X>X{ }_{g}$ or $\dot{x}=\dot{X} \cos \beta$ \\
\hline 4 & $\mathrm{X} \geqslant \mathrm{X}_{\mathrm{g}} ; \dot{\mathrm{x}}=\dot{\mathrm{X}} \cos \beta ;|\ddot{X} \cos \beta| \kappa \mu(g+\ddot{X} \sin \beta)$ & $X<X_{g}$ or $|\ddot{X} \cos \beta|>\mu(g+\ddot{X} \sin \beta) \mid$ \\
\hline 5 & $\begin{array}{l}X \geqslant X_{g} ; \dot{X}=\dot{X} \cos \beta ; \ddot{X} \cos \beta>\mu(g+\dot{X} \sin \beta)^{=1} \\
\text { or } X \geqslant X_{g} ; \dot{x}<\dot{X} \cos \beta\end{array}$ & $X<X_{E}$ or $\dot{X}=\dot{X} \cos \beta$ \\
\hline 6 & $\begin{array}{l}\mathrm{X} \geqslant \mathrm{X}_{\mathrm{g}} ; \dot{\mathrm{x}}=\dot{\mathrm{X}} \cos \beta ; \ddot{\mathrm{X}} \cos \beta<\mu\left(\mathrm{g}+\ddot{X}_{\sin } \beta\right) \\
\text { or } \mathrm{X} \geqslant \mathrm{X}_{\mathrm{g}} ; \dot{\mathrm{x}}>\dot{\mathrm{X}} \cos \beta\end{array}$ & $\mathrm{X}<\mathrm{X}_{\mathrm{g}}$ or $\dot{\mathrm{X}}=\dot{\mathrm{X}} \cos \beta$ \\
\hline
\end{tabular}

* on slip starting

on slip continuing while spring region changes.

5.5.I A modification to allow for particle motion when throwing occurs

The previous programme is only applicable to cases when the table maximum vartical acceleration $(\ddot{X}(t)$ sinp) aues nut exceed gravity so that contact between the table and the moving mass is not lost. This approach proved perfectly suitable for most cases which were experimentally investigated. However, if one was investigating the usual linear system, in which damping was low, it is probable that very close to the natural frequency of the system amplitudes would become large such that even at low frequencies, the maximum vertical acceleration 
would exceed that of gravity. The previous programme was thus extended to deal with this case, but only for a slider of negligible mass. The modification required would have been excessive, if sliders of mass comparable to that of the table had been considered. Further, if the slider mass is large, during slipping the Coulomb damping extracts energy from the system and so very large amplitudes are not so probable.

The first of equations (5.12) with $m=0$ describes the table motion in this case. In any practical system with $\beta \neq 90^{\circ}$ it is obvious that the slider will begin slipping before the vertical acceleration of the table causes it to be thrown. The equations of slider motion can thus be written (ref. 5.13 and 5.15)

$$
\begin{aligned}
\dot{x}(t) & =\dot{x}(t) \quad v=1 \text { or } 4 \\
& =\dot{x}(t-\tau) \pm N \mu(g+\ddot{x}(t) \sin \beta) \tau \\
v & =2,3,5,6
\end{aligned}
$$

where $N=I$ if the slider is in contact with the table, and $N=0$ if the slider has been thrown off and is in free fall. The condition leading to $N=0$ is that at some instant when $N=1, \ddot{X}\left(t_{I}\right)<-g$. This defines $t_{I}$ when the particle is thrown off the table surface. The trajectory of the particle in the vertical direction is given by

$$
y(t)=-\frac{1}{2} g\left(t-t_{1}\right)^{2}+\dot{Y}\left(t_{1}\right) \cdot\left(t-t_{1}\right)+Y\left(t_{1}\right) \quad \ldots 5.17
$$

and until $t_{2}$ such that $y\left(t_{2}\right)=Y\left(t_{2}\right)$ the horizontal velocity of the particle remains $\dot{x}\left(t_{1}\right)$. In general, for a meaninguul result to emerge $\left(t_{2}-t_{1}\right)$ must be less than the period of the vibration. In cases where the motion is harmonic, this implies that $\left(t_{2}-t_{1}\right)$ shall be less than the period of the forcing function.

The programme was modified to include $N$ as described above, with a jump instruction governed by vertical acceleration of the table and the particle vertical displacement. 


\section{5 .2}

The programme is included as an appendix to this section. Since the autocode used is particularly easy to follow no details of construction will be given. As previously mentioned, with the exception of a formula for slider inotion no numerical integration of differentiation was required, since all table displacements are twice differentiable functions, though the expression for table acceleration may be felt to be somewhat cumbersome. Several jump instructions which have not been previously mentioned will be found in the programme. These were included to avoid dividing by zero (and producing an infinity) if an exact root coincides with a value of $t$ used in the calculation. The programme was punched on 7-hole tape. Data, also transmitted on 7--hole tape, was grouped into batches each of which required the maximum programme time of 25-30 minutes. Thus one programme tape was used many times, and several hours work on data tapes ( 1 for each job) was sent off with the programme tape. The normal method adopted was to feed in a given spring characteristic and investigate the resulting travel rate over a frequency range covering the resonance peak. A sample of data input is shown after the programme. The data input is, in order: the number of frequencies to be used; the mass of the table; the mass of the slider; the stiffness of the soft spring; the stiffness of the hard spring; the amplitude of the force; the gap setting; the coefficient of friction (inserted as $\mu \mathrm{g}$ ); the damping factor; the angle of inclination (degrees); $I / g$; and a number used to identify the results with a particular data tape. Then follow the frequencies $(\mathrm{c} / \mathrm{s})$ which are to be calculated. The neans of inserting a linear characteristic is to make the gap setting very large and the hard spring stiffness 
equal to zero. It is worth noting that unlike the analogue computer investigation, in the numerical approach the value of the 'hard' spring stiffness used was the total restoring force in that region - i.e. the sum of the hard and soft spring stiffnesses of the real system.

The results which were computed in this way were again a parallel to the experimental work. All experiments carried out on system A (horizontal table motion with a particle slider) were repeated. No comparison was attempted with system B since coulomb damping would have been akward to insert into the programme. However, continuing with the constants of system $A$, the effect of making the mass being transported comparable to that of the conveyor was investigated. Results were obtained for the case when the system was vibrating in a plane at $\beta^{\circ}$ to the horizontal (the conveying surface being horizontal) for cases of both particle sliders (when the programme was valid for maximum accelerations greater than gravity) and sliders of mass comparable to that of the table. Both bi-linear and linear spring characterictics were studied.

A photograph of part of a print-out is shown, and figures show some results plotted out.

This complete programme required about 25 minutes to produce 10 results on K.D.F 9, a figure which might well be considered excessive for most users. As previouisly mentionied it should not be used in its complete form unless the full facilities of the programme are required. If any simplification such as $\beta=0$ or $m=0$ can be made, the programme should be re-written. It is a programme designed primarily for a system with a bi-linear restoring force, and it is not reasonable to expect it to be efficient for a linear system. While the programme no doubt contains many crudities which slightly impair its efficiency, 
in repeated use with many sets of data it proved most satisfactory and in its present form can be regarded as completely "de-bugged".

5.6 Further extensions which could be made to the programme.

The most obvious restriction on the programme is that it does not apply to cases where the slider mass is comparable to that of the table, and the table has a maximum vertical acceleration which exceeds gravity. It should be remembered that the term 'slider' which normally implies a single mass, is used here to denote any material being transported which behaves as a single rigid body. In a vibratory conveyor, motion is never only in the throwing region but always includes some slipping. When large masses are involved the resulting Coulomb damping may absorb energy and so limit accelerations. If the large mass takes the form of a bulk material, several revearchers have shown that the assumption of rigid body motion and free fall are not always valid. Until this point has been established by investigation into the behaviour of bulk materials it would not appear to be worth developing this aspect of the programme.

The mathematics of motion when the plane is at an angle $\not$ to the horizontal with the table vibrating at $\beta \geqslant 0^{\circ}$ to the horizontal has been covered in section 2. It will be seen that this would be a minor modification to the programme.

As described, the extraction of other data such as the work done by the force on the system is conveniently obtained when required. 


\section{DIGITAL COMPUTER PROGRAMME}

The following notation is used in the programme and differs from that used in the text.

$$
\begin{aligned}
& U(n)=\alpha(n) \\
& Y(n)=\dot{X}(n) \\
& \mathrm{C}(n)=\ddot{X}(n) \\
& W(n)=\dot{x}(n) \\
& Q=\mu g
\end{aligned}
$$

$R_{1}$ is the instant of changing regions

$R_{2}$ is the instant at which slipping starts

$$
Y t=\dot{X}\left(R_{2}\right)
$$$$
L=X\left(R_{1}\right)
$$$$
\mathrm{H}=\dot{\mathrm{X}}\left(\mathrm{R}_{1}\right)
$$$$
G=\ddot{X}\left(R_{1}\right) \text { or } \ddot{X}\left(R_{2}\right)
$$

$\mathrm{b}=\beta$

$\ell=$ damping factor

$a t, b t, c t, d t=t i m e$ required for initial approximation

$\mathrm{AA}, \mathrm{BB}, \mathrm{R}_{3}=$ are associated with throwing motion

$$
\mathrm{K}_{3}=\frac{1}{\text { gravity }}
$$

$$
\mathrm{g}=\text { gap setting }
$$

Other terms are either used in their previous content or are merely constants used for the computation.

The programme is shown in the form in which it was transmitted. 


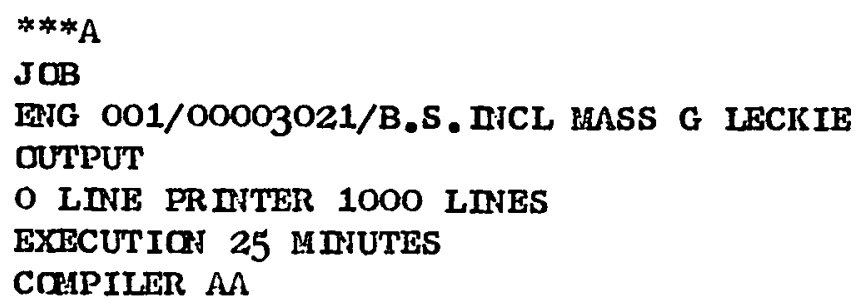

bogin

array $A(0: 6), B(0: 6), z(0: 6), U(0: 6), s(0: 6), p(0: 6), u(0: 6), c(0: 100), x(0: 100)$, $Y(0: 100), W(0: 100), V(0: 6)$

roal $M 1, H 2, K 1, K 2, F, g, Q, T, f, f f, x, f T, p t, R 1, R 2, Y t, G, L, H, a t, b t, c t, d t, 1, t$ ravol, $E, D, J, K 3, b, A A, B B, P, R 3$

intogor $k, j, i, n, m, v, r, b, t, r r, a$, tapo, $N$

road $(k, M 1, M 2, K 1, K 2, F, g, Q, 1, b, K 3$, tapo)

caption TAPIS

print(tapo, 1,0)

nowline

$b=b * \pi / 180$

$p(1)=$ sqrt $(\mathrm{Ir} 1 /(\mathrm{M} 1+\mathrm{M} 2))$

$\mathrm{p}(2)=\operatorname{sqrt}\left(\mathrm{K} 1 /\left(\mathrm{B} 1+\mathrm{M} 2 *(\sin (\mathrm{b}))^{2}+\mathrm{H} 2 * \mathrm{Q} * \mathrm{~K} 3 * \operatorname{ain}(\mathrm{b}) * \cos (\mathrm{b})\right)\right)$

$p(3)=\operatorname{sqrt}\left(K 1 /\left(M 1+M 2 *(\sin (b))^{2}-M 2 * Q * M 3 * \sin (b) * \cos (b)\right)\right)$

$p(4)=\operatorname{sqrt}(12 /(M 1+H 2))$

$p(5)=\operatorname{aqrt}\left(K 2 /\left(M 1+M 2 *(\operatorname{ain}(b))^{2}+M 2 * Q * 1 K 3 * \sin (b) * \cos (b)\right)\right)$

$p(6)=\operatorname{mrt}\left(\mathrm{K} 2 /\left(M 1+M 2 *(\sin (b))^{2}-M 2 * Q * K 3 * \sin (b) * \cos (b)\right)\right)$

$T=112 * 2 * \cos (b)$

cyclo $j=1,1, k$

$\operatorname{road}(f f)$

$f=f f * 2 \pi$

cyclo $i=1,1,3$

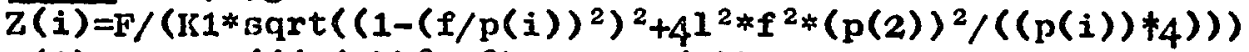

$U(i)=\arctan \left(\left((p(i))^{2}-f^{2}\right), 21 * f * p(2)\right)$

$\operatorname{print}(\mathrm{z}(i), 1,3) ; \operatorname{print}(\mathrm{u}(\mathrm{i}), 3,3)$

ropoet

If $\mathrm{K} 2=0$ thon $\rightarrow 72$

cycie $i=4,1,6$

$Z(i)=F /\left(12 * 3 q r t\left(\left(1-(f / p(i))^{2}\right)^{2}+4^{2} * f^{2 *}(p(2))^{2} /((p(1)) * 4)\right)\right)$

$U(i)=\arctan \left(\left((p(i))^{2}-f^{2}\right), 21 * f * p(2)\right)$

print(z(i),1,3); print $\|^{\prime}(U(i), 3,3)$

ropoat

$\mathrm{s}(4)=\mathrm{g}-\mathrm{K} 1 / \mathrm{IK} 2 * \mathrm{~g}-\mathrm{R} 2 / \mathrm{K} 2 * 386 * \mathrm{~B}$ in $(\mathrm{b}) \| \mathrm{i} \mathrm{S}(5)=\mathrm{S}(4)-\mathrm{T} / \mathrm{K} 2 ; \mathrm{s}(6)=\mathrm{S}(4)+\mathrm{T} / \mathrm{K} 2$

72: $\quad \mathrm{s}(1)=-\mathrm{K} 2 / \mathrm{K} 1 * 386 * \sin (\mathrm{b}) ; \mathrm{s}(2)=-\mathrm{T} / \mathrm{K} 1+6(1) ; \mathrm{s}(3)=\mathrm{S}(1)+\mathrm{T} / \mathrm{K} 1$

$u(1)=0 ; u(2)=0 ; u(3)=-0 ; u(4)=0 ; u(5)=0 ; u(6)=-0$

$v=1$ 
$\mathrm{N}=1 ; \mathrm{x}=.02 \pi$

if $112=0$ thon $\rightarrow 70$

$a t=\pi / 2 * f / p(4) ; b t=2(\pi-a t) * p(1) / f ; c t=\pi / 2-a t ; d t=\pi / 2+a t$
$A(v)=g-Z(v) * \sin (d t-U(v))+s(v)$
$B(v)=(-\Lambda(v) * \cos (b t)+g-Z(v) * \sin (c t-U(v))) / \sin (b t)$
$R 1=d t-27 ; x=.02 \pi$
$\rightarrow>71$

70: cyclo $n=1,1,6 ; n(n)=0 ; B(n)=0 ;$ ropoat

71: cyclo $n=0,1,60$

1: if $n>56$ thon $\rightarrow 51$

2: cyclo $\mathrm{n}=0,1,100$

11: $\quad f T=2 ; * n+m^{* * x}$

$p t=p(v) / f *(P T-R 1)$

$J=\operatorname{oxp}(-1 * p(2) *(f T-R 1) / f)$

$C(m)=J *(-\Lambda(v) * \cos (p t)-B(v) * \sin (p t)) *(p(v))^{2}-21 * p(2) * p(v) * J *$ $(-A(v) * \sin (p t)+B(v) * \cos (p t))+1^{2} *(p(2))^{2} * J *(A(v) * \cos (p t)+$ $B(v) * \sin (p t))-Z(v) * f^{2} * \sin (f T-U(v))$

$X(n)=J *(A(v) * \cos (p t)+B(v) * \sin (p t))+Z(v) * \sin (f T-U(v))+S(v)$

$Y(m)=J *(-A(v) * \operatorname{Bin}(p t)+B(v) * \cos (p t)) *(p(v))-1 * p(2) * J *(A(v) * \cos (p t)+$ $B(v) * \sin (p t))+Z(v) * f * \cos (f T-U(v))$

$W(m)=Y(m) * \cos (b)$

if $v=1$ or $v=4$ then $\rightarrow 15$

if $\mathrm{m}=0$ thon $\rightarrow 45$

if $\mathrm{n}=\mathrm{a}$ thon $\rightarrow 44$

if $\mathrm{N}=1$ thon $\rightarrow 103$

$\rightarrow 100$

103: if $\mathrm{C}(\mathrm{m}) * \mathrm{Bin}(\mathrm{b})<-386$ thon $\rightarrow 98$

101: $\quad W(m)=W(m-1)+u(v) * x / f * \overline{(1, \mid)}+C(m) * K 3 * \sin (b)) * N$ $\rightarrow 15$

44: $\mathrm{W}(\mathrm{m})=\mathrm{Y} t+\mathrm{U}(\mathrm{v}) *(\mathrm{fT}-\mathrm{R} 2) / \mathrm{f} *(1+\mathrm{K} 3 * \mathrm{C}(\mathrm{m}) * \mathrm{Bin}(\mathrm{b})) * \mathrm{~W}$ $\rightarrow 15$

45: $W(m)=W(100)$

15: if $m=0$ thon $\rightarrow 10$

if $n=r r$ thon $\rightarrow 40$

41: if $m=r$ thon $\rightarrow 10$

if $\mathrm{m}=\mathrm{t}$ thon $\rightarrow 12$

if $m=t+1$ thon $t=0$

if $! x(m)-g !<=0$ !

if $(\mathrm{x}(\mathrm{m}-1)-\mathrm{g}) /(\mathrm{x}(\mathrm{m})-\mathrm{g})<0$ thon $\rightarrow 20$

13: if $v=1$ or $v=4$ thon $\rightarrow 9$

if $|W(\mathrm{~m}) \overline{-Y}(\mathrm{~m}) * \cos \overline{(\mathrm{b})}|<.000001$ thon $\rightarrow 7$

if $(\mathrm{W}(\mathrm{m})-\mathrm{Y}(\mathrm{m}) * \cos (\mathrm{b})) /(\mathrm{W}(\mathrm{m}-1)-\mathrm{Y}(\mathrm{m}-1) * \cos (\mathrm{b}))<0$ thon $\rightarrow 21$ $\rightarrow 10$

9: $\quad H(m)=Y(m) * \cos (b)$

if $C(n) * \cos (b)>a *(1+1 K 3 * C(n) * \sin (b))$ thon $\rightarrow 22$

if $c(m) * \cos (b)<-n *\left(1+K 3^{*} \mathrm{C}(\mathrm{m}) * \operatorname{Bin}(\mathrm{b})\right)$ thon $\rightarrow 23$

10: ropoat

ropoat

$\rightarrow 51$

40: if $v=1$ or $v=4$ thon $\rightarrow 41$ 
if $v=2$ or $v=5$ thon $\rightarrow 42$

if $W(\mathrm{~m})>\bar{Y}(\mathrm{~m}) * \overline{c o s}(\mathrm{~b})$ thon $\rightarrow 41$

$\mathrm{v}=\mathrm{v}-2$

$\mathbf{r} \mathbf{r}=0$

$\rightarrow 24$

42: if $W(m)<Y(m) * \cos (b)$ thon $\rightarrow 41$

$v=v-1$

$\mathrm{rr}=0$

$\rightarrow 24$

98: $\quad \mathrm{BB}=\mathrm{X}(\mathrm{n}) *_{\operatorname{Bin}} \mathrm{B}(\mathrm{b})$

$\mathrm{N}=0$

R3 $=\mathbf{f T}$

$P=Y(n) * \operatorname{Bin}(b)$

$->101$

100: $\Omega A=B B+P *(f T-R 3) / f-193((f T-R 3) / f)^{2}$

if $A A>X(m) * \operatorname{Bin}(b)$ thon $\rightarrow 101$

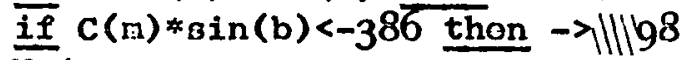

$\mathrm{N}=1$

$\rightarrow 101$

5: $\quad \mathrm{IT}=2 \pi * \mathrm{n}+\mathrm{n} * \mathrm{x}$

$\mathrm{pt}=\mathrm{p}(\mathrm{v}) / \mathrm{f} *(\mathrm{fT}-\mathrm{R} 1)$

$t=m+1$

$\rightarrow 6$

12: if $(x(m)-g) /(x(m-2)-g)>0$ then $\rightarrow 5$

$\rightarrow 13$

7: $\quad \mathrm{IT}=2 \pi^{*} \mathrm{n}+\pi^{* * x}$

$\rightarrow 8$

20: $\quad f T=2 \pi * n+(m-1) * x+(|x(n-1)-g|) /(|x(m-1)-g|+|x(m)-g|) * x$

6: $\quad p t=p(v) / f *(f T-R 1)$

$J=\exp (-1 * \mathrm{p}(2) *(f \mathrm{~T}-\mathrm{R} 1) / \mathrm{f})$

$\mathrm{L}=\mathrm{g}$

$H=J *(-A(v) * \sin (p t)+B(v) * \cos (p t)) * p(v)-1 * p(2) * J *(A(v) * \cos (p t)+B(v) *$ $\sin (p t))+Z(v) * f * \cos (f T-U(v))$

$G=(-A(v) * \cos (p t)-B(v) * \sin (p t)) *(p(v))^{2} * J-21 * p(2) * p(v) * J *(-A(v) * \sin (p t)$ $+B(v) * \cos (p t))+I^{2} *(p(2))^{2} * J *(A(v) * \cos (p t)+B(v) * B \operatorname{in}(p t))-$ $Z(v) * f^{2 *} \operatorname{Bin}(f T-u(v))$

if $v=1$ or $v=4$ thon $\rightarrow 30$

$\bar{x}=\overline{\mathrm{LiU}}$

if $(W(m)-Y(m) * \cos (b)) /(W(m-1)-Y(m-1) * \cos (b))>0$ then $\rightarrow 30$

$\overline{G=}(-A(v) * \cos (p t)-B(v) * \sin (p t)) *(p(v))^{2} * J-21 * p(2) * p(v) * J *$

$\left.(-A(v) * \operatorname{Bin}(p t)+B(v) * \cos (p t))+1^{2 *}(p(2))\right)^{2} * J *(A(v) * \cos (p t)+$ $B(v) * B \operatorname{in}(p t))-Z(v) * f^{2} * \sin (f T-U(v))$

if $v=2$ or $v=5$ thon $\rightarrow 33$

if $G * \cos (b)>(2 *(1+K 3 * G * \sin (b))$ thon $\rightarrow 34$

$\mathrm{v}=\mathrm{v}-2$

$\rightarrow 30$

34: $v=v-1$

$\rightarrow 36$ 


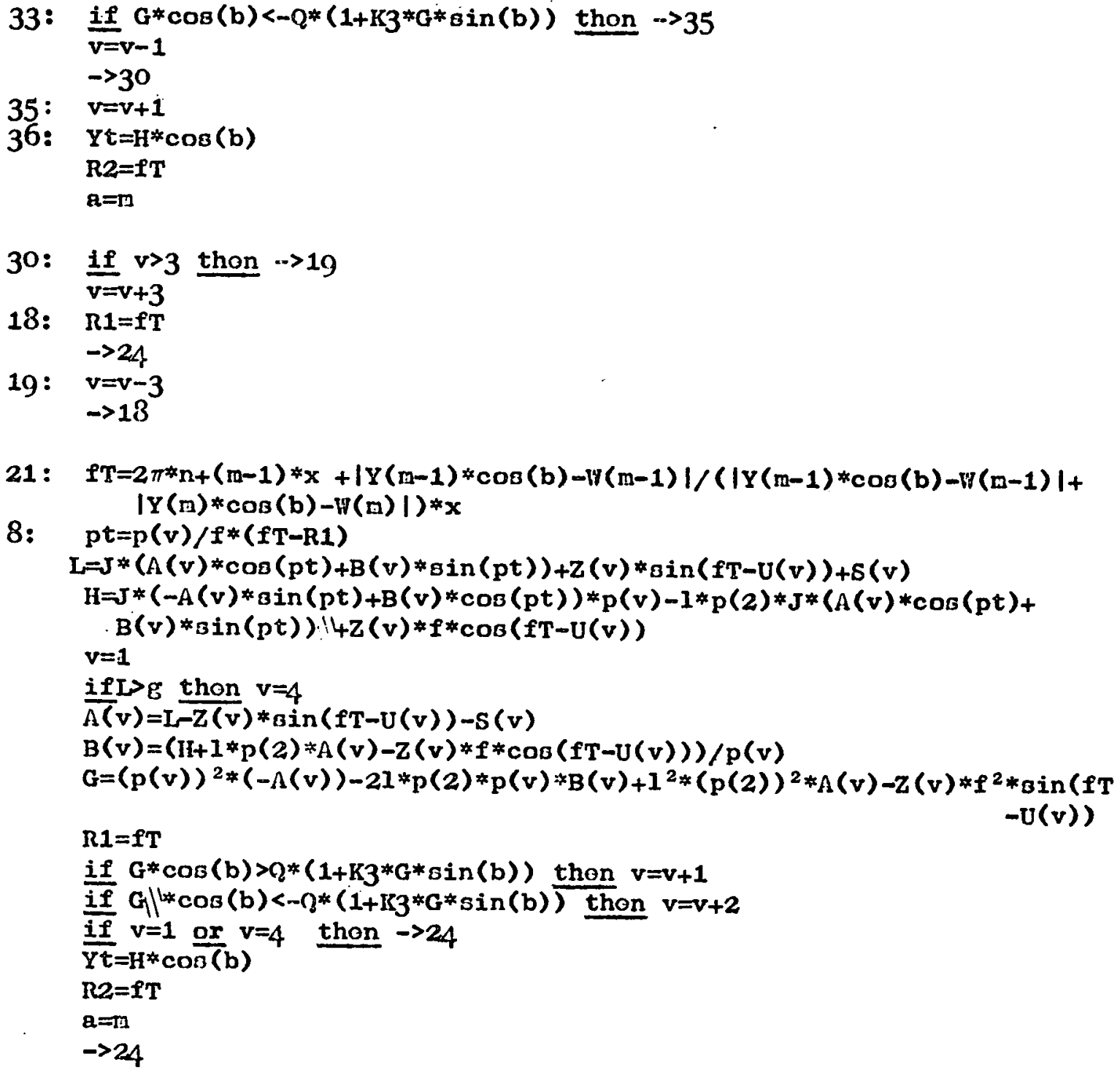

22: $\mathrm{fT}=2 \pi * n+(m-1) * x+\left|c(n-1) * \cos (b)-n *\left(1+K 3^{*} \mathrm{C}(\mathrm{m}-1) * \sin (b)||\right)\right| /$

$(|\mathrm{c}(\mathrm{m}) * \cos (\mathrm{b})-\mathrm{Q} *(1+\mathrm{K} 3 * \mathrm{C}(\mathrm{m}) * \sin (\mathrm{b}))|+\mid \mathrm{C}(\mathrm{m}-1) * \cos (\mathrm{b})$ $\left.-0 *\left(1+K 3^{*} C(m-1) * \sin (b)\right) \mid\right) * x$

53: $\quad p t=p(v) / f *(f T-R 1)$

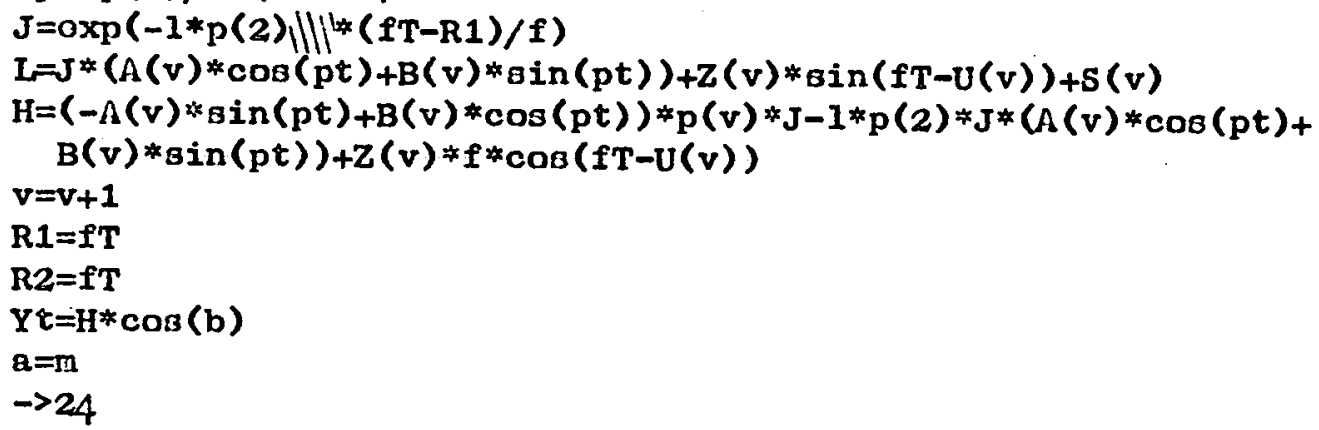


23: $\quad f T=2 \pi * n+(m-1) * x+|c(m-1) * \cos (b)+Q *(1+K 3 * C(m-1) * \sin (b))| /$ $(|\mathrm{C}(\mathrm{m}-1) * \cos (\mathrm{b})+\mathrm{Q} *(1+\mathrm{K} 3 * \mathrm{C}(\mathrm{m}-1) * \sin (\mathrm{b}))|+\mid \mathrm{C}(\mathrm{m}) * \cos (\mathrm{b})+$ $\left.\mathrm{Q} *\left(1+\mathrm{K} 3^{*} \mathrm{C}(\mathrm{n}) * \sin (\mathrm{b})\right) 1\right) * x$ $p t=p(v) / f *(f T-R 1)$

$I=J *(A(v) * \cos (p t)+B(v) * \sin (p t))+Z(v) * \sin (f T-U(v))+S(v)$

$H=(-\Lambda(v) * \sin (p t)+B(v) * \cos (p t)) * p(v) * J-1 * p(2) * J *(A(v) * \cos (p t)+$ $B(v) * \sin (p t))+Z(v) * \cos (f T-U(v)) * f$

$\mathrm{v}=\mathrm{v}+2$

$R 1=f T$

$\mathrm{R} 2=\mathrm{fT}$

$\mathrm{Yt}=\mathrm{H}^{*} \cos (\mathrm{b})$

$a=n$

$->24$

24: $\Lambda(v)=L-Z(v) * \sin (f T-U(v))-S(v)$

$B(v)=(H+1 * p(2) * A(v)-Z(v) * f * \cos (f T-U(v))) / p(v)$

$I=\mathrm{n}$

$\rightarrow 11$

51: nowlino

print $(f f, 2,2) ; \quad$ opacos(2); $\operatorname{print}(g, 1,3)$

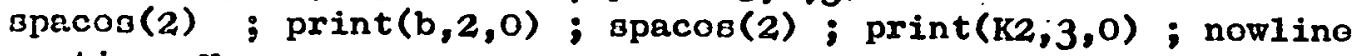
caption $x$

cyc10 $s=0,5,100$

print $(x(\pi), 1,3)$

ropoat

nowlino

caption $Y$

cyclo $8=0,5,100$

print $(Y(B) * \cos (b), 2,2)$

ropoat

nowline

caption $\mathrm{H}$

cyc10 $s=0,5,100$

print $(W(B), 2,2)$

ropoat

nowlino

$\overline{\mathrm{E}}=\overline{\mathrm{O}} ; \overline{\mathrm{D}}=\overline{\mathrm{O}}$

cyc10 $3=1,2,99$

$E=W(B)-Y(B) * \cos (b)+E$

ropoat

cyclo $8=2,2,98$

$D=W(B)-Y(B) * \cos (b)+D$

ropoat

travol $=.00333 / f f *(H(0)+W(100)-Y(0) * \cos (b)-Y(100) * \cos (b)+2 E+4 D)$

caption TRAVEL \& RATE

print(travol, 1,3)

caption INS \& PER \& CYCLE 
print (ff*travol, 3,3$)$

if $\mid$ travol|>.0001 thon $\operatorname{print}(1 /(f f * t r a v o 1), 3,3)$

if $n=60$ thon $\rightarrow 4$

$->2$

4: ropoat

ond of progran

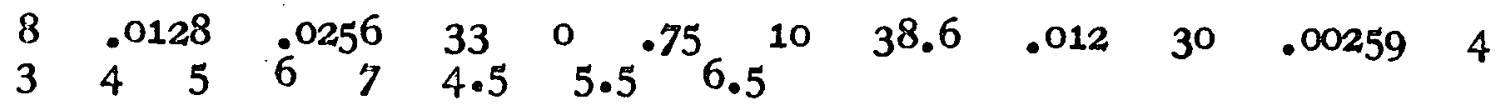

$* * * 2$ 


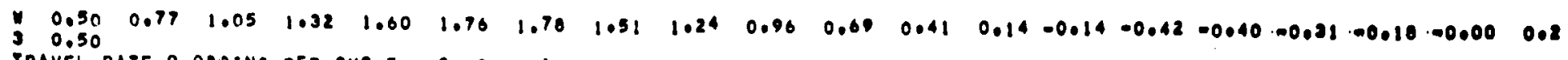
TRAVEL RATE 0.000 INS PER CYCLE $0.020 \quad 1.599$

MORK DONE 0.05 JIN LBS PER CYCLE 0.351

$7.00 \quad 0.025$

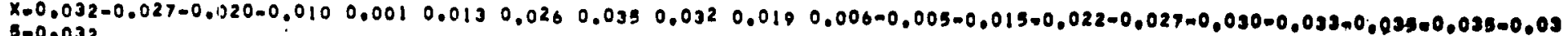
300.032

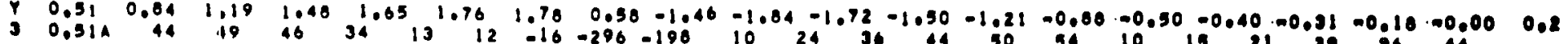

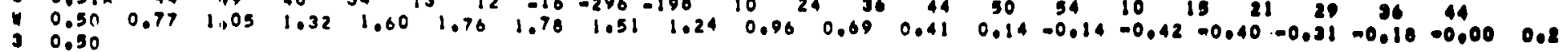

PRAVEL RATE 0.081 INS PER CYCLE $0.626 \quad 1.390$
MORK DONE 0.031 IN LBS PER CYCLE 0.351

$.7 .00 \quad 0.025$

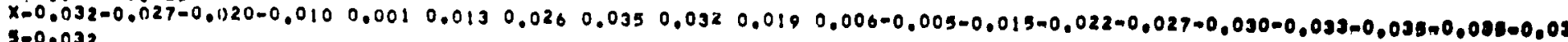

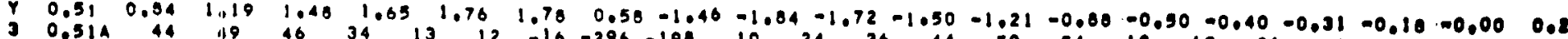

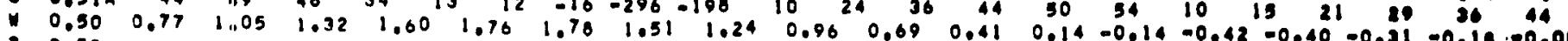

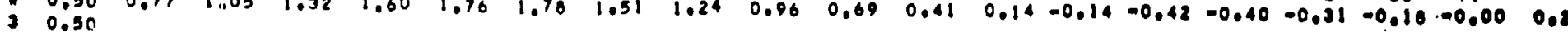

MORK DONE $0.0501 N$ PER CYCLE $0.626 \quad 1.590$

$7.00 \quad 0.025$

$x=0.032-0.027-0.0120-0.010 \quad 0.001$
5 $5-0.032$

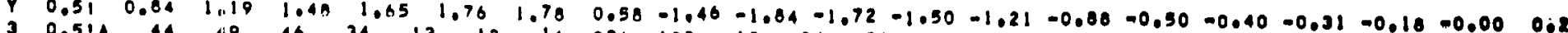

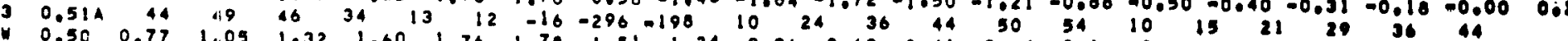

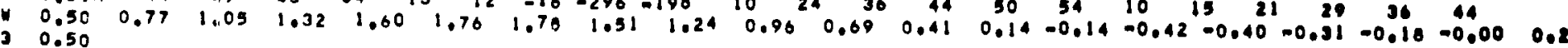

RPREL RATE 0.005 INS PER CYCLE 0.6261 .590

SPRK DOHE 0.05CIN LAS PER CYCLE 0.351

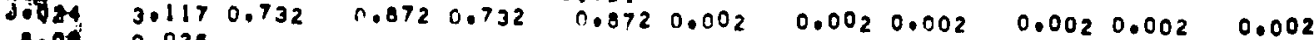

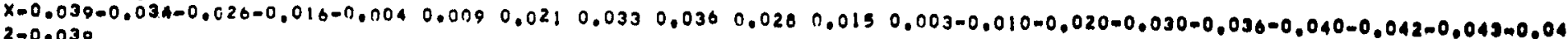

$2-0.030$

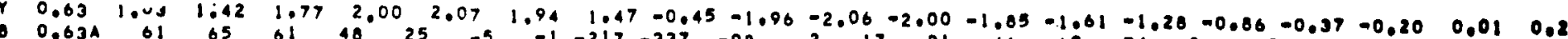

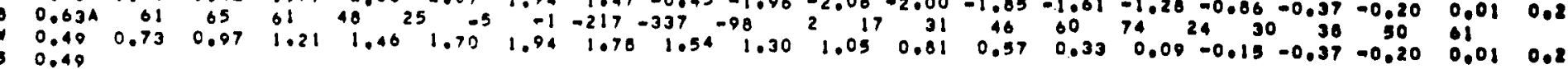

TRAVEL RATE 0.097 INS PER CYCLE $0.776 \quad 1.280$

MORK DONE $0.067 I N$ LES PER CYCLE 0.536

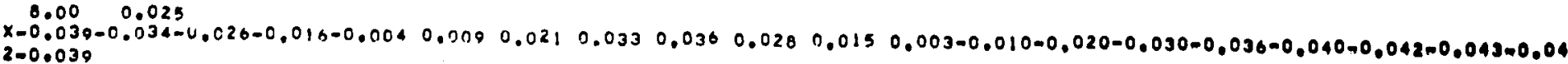
$2=0.039$

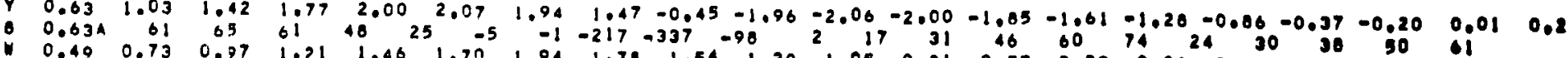

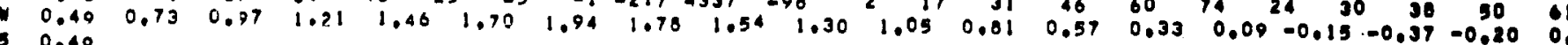

TRAYRL RATE 0.0971 IAS PEQ CYCLE $0.776 \quad 1.289$

MORK ONNE $0.0671 \mathrm{~N}$ LBS PER CYCLE 0.536

$0.00 \quad 0.025$

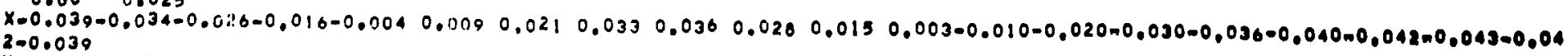

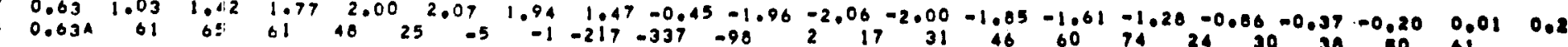

Part of print-out from digital computer. 


\section{CHAPTER 6}

\section{RESULTS AIND DISCUSSION}

All the results are shown graphically at the end of this chapter. Since no set of non-dimensional parameters could be established which would allow presentation of results in a general form, the results are shom in the form in which they were taken experimentally. This absence of suitable non-dimensional representation is an immediate difference between vibratory conveyors with non-Iinear and linear spring force characteristics. It has previously been mentioned however, that in the latter case it is usual to treat amplitude as an independent variable which is not the case in a resonant vibratory conveyor. In some work on non-linear systems, and particularly those where the non-linearity is in the form of a cubic term in the restoring force a 'percentage non-linearity' can be derived as a function of maximum amplitude. While this is possible for a quasi-linear system it is not useful when the non-linearity is unsymmetrical or severe, when, as was mentioned in Chapter 2, it becomes meaningless to talk about amplitude of a waveform. Finally, the performance is represented as a 'travel rate' in seconds/inch rather than as a velocity in ins./second. It was felt that the former was more useful when slow transport rates were involved.

\section{I Results from System A}

Figures $6.1-6.3$ show response curves obtained from the analogue computer for various non-linear spring characteristics. It is obvious that the linear system was very lightly damped, and that very large amplitudes could occur at resonance. The non-linearity in these, and in all other graphs, was denoted by the stiffness of the 'impact' spring and the 'gap' setting. It will be seen that the response curves for 
the non-linear spring characteristics are not complete. At lower frequencies the response curve of a non-linear system would, in general have a common point with the response curve of the linear system at a frequency where the latter had an amplitude equal to the gap setting. In the present case however, at and immediately above this frequency the response to forced vibration is complicated by the presence of subharmonic response and complex waveforms where the term 'amplitude' has no real meaning. At higher frequencies no non-linear 'jump' is shown, due to the limit on the frequency of excitation which could be achieved without causing over-travel of the vibrator. The jump frequencies were found by analogue computer to be between $15 \mathrm{c} / \mathrm{s}\left(K_{2}=360 \mathrm{lb} /\right.$ ins.; gap 0 ins $)$ and $11 \mathrm{c} / \mathrm{s}\left(K_{2}=90 \mathrm{lb} /\right.$ ins.; gap .050 ins $)$. With zero gap after this jump, the displacement was still non-linear, whereas for positive gap settings the system reverted to linear response with an amplitude less than the gap setting. Figures $5.1-6.3$ show an expected trend in that as the gap is increased, and the hard spring stirfness decreased, the response begins to approach that of the linear system.

Figures 6.4 to 6.10, which contain 9 graphs in all, show the travel rates for a brass slider $(\mu=.12)$ against frequency, the exciting force being .75 sinut. Results shown are those obtained by experimental methods, from the analogue computer circuit, from the digital computer programme, and in Figure 6.7 from waveforms calculated by the approximate method of chapter 5. The legend $\frac{1}{3}(4)$, for example, indicates a conveyor motion which has a period which is three times that of the forcing function, and during this limit cycle, contact with the hard spring occurs four times.

Reference to Figure 6.4 shows that the results are a great deal more complicated than those for a linear conveyor. While motions of 
the type $1 / 1$ ( ) have the same period as the forcing function, and as such would usually be termed 'harmonic' solutions, there are no less than 6 regions where such a solution exists, with no indication that these regions are part of a single curve. Another feature which would not be found in a linear system is the presence of motions whose period is 2, 3, or 4 times that of the forcing function. According to the definitions which appear in most standard works, such motions are designated $\frac{1}{2}$, $\frac{1}{3}$ or $\frac{1}{4}$ subharmonics. This definition shall be maintained for the present, and the subject explored more fully in Appendix E.

Figures 6.5 and 6.6 show a similar pattern of harmonic and subharmonic response as was found in Fig. 6.4. Although several of the small regions of harmonic and sub-harmonic response give fairly good travel rates, only that region of harmonic response at frequencies greater than those at which subharmonic response is found are likely to be of much practical interest, for at lower frequencies a small change in exciting frequency might result in a change of type of motion with a resulting large change in travel rate. In this upper region of harmonic response a turning point exists above which the travel rate decreases as the frequency of excitation increases, this effect being most rarked for the softest 'impact' spring. It has been shown in chapter 2 that horizontal sinusoidal displacement will not produce material transport in the absence of a vertical component. The motion is of the continuous slip type, the relative forward displacement being cancelled by an equal and opposite backwards displacement. As the conveyor amplitude increases so the waveform approaches the linear case and the resulting transport rate tends to zero. The trend which Figs. 6.4, 6.5, 6.6 show is that as the stiffness of the impact spring decreases, so also does the maximum travel rate, though only slightly. The frequency at which this maximum 
occurs also decreases, and the range over which travel rate is reasonably independent of frequency becomes smaller.

Figures 6.7 and 6.8 are for the same impact springs with a gap setting of .025 ins. The curves are somewhat simpler than the previous ones in that only two regions of harmonic solutions exist, and that subharmonic response is found only at one frequency with $K_{2}=360 \mathrm{lb} / \mathrm{in}$. A similar trend is found as in the previous case with the stiffest spring having a slightly faster maximum travel rate than the others, the frequency at which it occurs being greater, as is the frequency band over which the fastest travel rates occur.

Figures 6.9 and 6.10 show the travel rates for the same three springs when the gap is $.050 \mathrm{ins}$. Fig. 6.9 shows a return to a more complicated response. The harmonic solution at $4.5-4.75 \mathrm{c} / \mathrm{s}$ corresponds to 'hard excitation'. At this frequency the response of the linear system is less than .050 ins, and the motion with larger amplitudes must be started by giving it an initial impulse. It should be noted that in these graphs the absence of a solution of any given type does not mean that it does not exist, but merely that it does not produce significant travel rates. In the upper graph of Fig. $6.10\left(\mathrm{~K}_{2}=190 \mathrm{lb} / \mathrm{in}\right)$ a region $\frac{1}{3}(4)$ was found at $7.5 \mathrm{c} / \mathrm{s}$, though the resulting travel rates were very low. The trend of the graphs is as was described for the other gap settings. It is noticeable that increasing gap does not have a marked effect on the travel rate for a given 'impact' spring. Little or no effect is shown with $K_{2}=360 \mathrm{lb} / \mathrm{in}$., and only a slight falling-off takes place with $\mathrm{K}_{2}=90 \mathrm{lb} / \mathrm{in}$. This indicates that a stiff spring should be used, the exact choice depending on how it affects the production of other regions of harmonic or subharmonic response. Increasing gap may occasionally remove various types of solution, but it should be 
remembered that this will reduce the 'jump' frequency. Iilile a marked change may result in travel rate if a sub-harmonic motion is picked up, if the system is pushed over the 'jump' frequency, no travel rate at all is the likely outcome.

The degree of agreement between the solutions obtained by various methods was most encouraging, and provides ample proof of their accuracy. At higher frequencies experimental travel rates were usually faster than those found by other methods. In chapter 3 some possible explanations were advanced for this. When the table is executing large amplitude vibrations, starting conditions for timing are more difficult. With large amplitude motion with relative sliding of the 'continuous slip' type, any variation in friction force by local surface irregularities, or by 'directional preference' as described in Appendix D can cause large changes in the time to cover short distances.

\subsection{Results obtained from System B}

The results for System B are shown in Figures 6.11-6.27. No digital computer solutions were attempted for this particular system due to the inconvenience in altering the digital computer programme to include coulomb damping.

Figures 6.11 - 6.13 show response curves for System B, which has much higher damping than was present in System A. In this case the nonlinear resonance 'jumps' are shown. It is particularly interesting to note that while the cases with positive gap settings drop to the linear resporise curve after resonance, the case of zero gap behaves rather differently. It is assumed that the left and right hand curves either meet or that there is an amplitude jump at a certain frequency. The immediate region of this change is very erratic and could not be investigated closely. 
Figures $6.14-6.19$ show the variation in travel rate against frequency for various spring non-linearities, with the table vibrating in a horizontal plane. The increased damping had a striking effect on regions of harmonic and subharmonic response. A harmonic response with two 'impacts' per cycle persisted, but only the $\frac{1}{2}$ sub-harmonic remained, and that in much more restricted regions. The heavier damping had the effect of reducing amplitudes and hence travel rates. In all cases the gap setting of .025 ins gave the best compromise between good travel rates and large frequency bandwidth over which frequency is relatively unimportant in maintaining transport. The trends established for System $A$ were still noticed, stiffer springs giving better maximum travel rates at higher frequencies, with a broader operating frequency band than is the case for softer springs. Figure 6.20 shows that when the hard 'impact' spring is set at zero gap transport continues over the resonance 'jump', though it should be noted that this jump produces a very erratic region which in a practical system would prohibit any attempt to use both portions of the graph.

System $B$ was constructed nainly to investigate the effects of inclining the conveyor motion at an angle to give horizontal and vertical components. The horizontal case which was shown in Figs. 6.14 - 6.19 proved a convenient starting point, for Figs. 6.21 and 6.22 show the effects of inclining the conveyor motion. These graphs are ropresentative of the behaviour found for a great many results. For the case with $\mathrm{K}_{2}=360 \mathrm{lb} / \mathrm{in}$. with zero gap only the fastest travel rates exhibited any improvement as the angle of inclination was increased, and in general a decrease in transport rate occurred. With a gap setting of .025 ins. the travel rate proved more or less independent of inclination, and only slight improvement was achieved when the gap setting was .050 ins. 
By constrast, the effect of inclination on the linear system, Fig. 6.23, shows that the conveyor has no travel rate unless there is some inclination, and that the travel rate increases as the angle of inclination is increased. For the linear system the improvement in travel rate through the effect of the vertical acceleration on the slider velocity more than offsets the loss of horizontal velocity, while the opposite is the case for the non-linear system.

Fig. 6.24 shows the travel rate of the linear system against frequency for an angle of inclination of $30^{\circ}$. While very fast travel occurs at resonance there are few frequencies at which a change in exciting Irequency of 1 cycle/sec would not change the travel rate by at least a factor of two. This compares unfavourably with results shown in Fig. 6.15 (gap .025 ins) for example when a change in frequency from $8.5 \mathrm{c} / \mathrm{s}$ to $11 \mathrm{c} / \mathrm{s}$ changes the travel rate from $2.4 \mathrm{secs} / \mathrm{ins}$. to 1.75 secs/ins.

Figures $6.25-6.27$ allow comparison between the linear system and the various non-linear configurations used when the angle of inclination is $30^{\circ}$. In some cases the non-linear systems produce throwing while at $30^{\circ}$ the linear system at the resonant frequency is at its limiting case. Hhile motion at an angle is not favourable to the nonlinear systems there are ranges on all curves at which reasonable travel rates can be obtained and where the travel rate is less dependent on accurate control of the frequency of the exsiting force than is the case for the linear system.

The results of Fig. 6.25 were taken from the analogue computer in order to establish the best operating range of angle of inclination of vibration for a linear system. The acceleration was monitored and the exciting force adjusted to give a maximum of ' $g$ '. This figure indicates 
that the best operating range is $40^{\circ}$ to $70^{\circ}$.

The agreement between the analogue computer results and the experimental results was not so good for this System as for system A. The main discrepancy takes the form of a frequency shift between the results of $\frac{1}{2}$ cycle/sec, those cases with zero gap being particularly affected. This could be caused either by inaccurate gap setting in the experimental system, caused either by a D.C. component in the force, or by a less sensitive equilibrium position when coulomb damping was high, or by the inaccuracy of the relays controlling the coulomb damping term on the SC 30 computer.

Thile a linear vibratory conveyor depends on an inclined path of conveyor vibration of $40^{\circ}$ or more, it is shown that such motion is detrimental to the performance of a non-linear conveyor of the type used in this experiment.

\subsection{Further results obtained by computer}

Though the experimental range could not be extended easily, it was possible to extend the scope of the project to include cases where the weight of material being transported was taken into account, using the analogue and digital computers. The representation of System B was not attempted due to the inconvenience of including coulomb damping in the digital computer programme, so the coefficients of System A were used.

Fig. 6.29 shows the variation of travel rate with coeficient of friction for a linear vibrator excited at $30^{\circ}$ to the horizontal. At resonance, throwing occurs. Near resonance where amplitudes are large, higher coefficients of friction give better travel rates, but low values of $\mu$ give faster travel where amplitudes are small. This effect is discussed in other works $(4,30)$. At $7.2 \mathrm{c} / \mathrm{s}$ the travel rate 
is reasonably independent of $\mu$ in the region $.1 \leq \mu \leq .5$.

Fig. 6.30 shows the de-tuning of a linear system which results when the weight of material being transported becomes large: Exactly $6 \mathrm{c} / \mathrm{s}$ is the only frequency at which the conveyor would continue to transport material loads varying from zero to two times the weight of the conveyor itself with a coefficient of friction $\mu=.1$. Other results showed that with $M_{2}=M_{1}$ and $\mu \geqslant .3$ only very smalI frequency bands existed at which transport could occur. The limitations of a linear system for transporting heavy loads is clearly indicated. . Redford $(30)$ found similar effects, and Fig. 6.37 shows the change in response of a bowl conveyor.

Fig. 6.3I shows the dependence of the performance of a horizontal non-linear conveyor on change in the coefficient of iriction.

Fig. 6.32, which co5responds to Fig. 6.30, indicates that the nonlinear system is less affected by material load than is the linear system. These two cases are directly compared on Fig. 6.33. Tor the particular non-linearity show travel rates occur for material loads from zero to twice that of the conveyor between $5 \mathrm{c} / \mathrm{s}$ and $7 \mathrm{c} / \mathrm{s}$, and are almost independent of material load between $6 \mathrm{c} / \mathrm{s}$ and $7 \mathrm{c} / \mathrm{s}$, while the linear system is shown to be highly effected by material load. This is obviously a powerful argument in favour of non-linear conveyors. The variation in travel rate with $\mu$ when $M_{2}=M_{1}$ is shown in Fig. 6.34, lower values of $\mu$ giving better travel rates.

In chapter 5 it was mentioned that the energy requirements of a vibratory conveyor could conveniently be extracted from the digital computer, and Fig. 6.35 shows the displacement/unit work for a conveyor whose material mass is equal to that of the conveyor. For simple sliding the maximum displacement per unit work is $1 / \mu \mathrm{mg}$. When double 
sided slip occurs nore energy is extracted from the system and the efficiency falls. In other cases the only loss of energy is caused by damping in the system and the work done against friction. In chapter 2 it was stated that continuous slip introduced a form of coulomb damping but no other changes in the case of a horizontal non-linear conveyor, and that damping has little effect on anplitude in many non-linear systems. Thus the travel rate might be expected not to be very dependent on coefficient of friction or material mass. The waveforms shown in Fig. 6.35 indicate how a phase-shift is the principal effect of the additional material damping. Calculations of work done/cycle were only carried out for the horizontal non-..linear conveyor. They would perhaps have been of interest for the linear conveyor inclined at an angle when transporting heavy material, when the effect of the vertical component would help to cancel the effects of continuous slip.

Pig. 6.36 shows waveforms of a case when throwing occurs, the result being obtained by digital computer as discussed in chapter 5 . The travel rates from this motion are show in Fig. 6.30 .

It will be noticed that no graphs have been shown for the case of comparable mass for the non-linear case when the oscillation is at an angle to the horizontal. The digital computer results for inclined oscillation and negligible material mass showed the same lack of dependence on angle of inclination as did previous results. With the spring characteristic defined as bilinear, and the gap set with reference to the conveyor in its unloaded equilibrium position, a new equilibrium position of the conveyor, and hence the gap setting, was caused by the material load. In the system used in this thesis the increase in gap setting due to a material load equal to the weight of the conveyor caused the system to degenerate to the linear case for $\beta=30^{\circ}$. 
There is, of course, no reason why the gap setting could not be defined with reference to the expected material load or even why a different form of non-linearity should not be used.

The results of this section showed good agreement between analogue and digital computer solutions.

\subsection{Plates and additional Figures}

Records of the output of various signals were taken from the analogue computer either by five channel U.V. recorder or on an $X-Y$ plotter. Plates $6.1-6.8$ are copies of these and illustrate various types of motion.

Plate 6.1 is for a linear systen such as was illustrated in Fig. 2.2. Zero lines were shown on the output from the U.V. recorder but these have been lost in obtaining better contrast for reproduction. The velocity traces are the super position of two signals - not as might be supposed those of $\dot{X}$ and $\dot{x}$, but an upper and lower half. The upper of the two traces, either $\dot{X}$ or $\dot{x}$, constitutes one signal, the lower, the other signal It can be seen where the 247 computer was set to 'Compute' to give $x$, the slider displacement, as a time increasing function.

Plate 6.2 is for System $B$ and shows how the efiect of increasing angle is to change double sided slip (Trace 2) into single sided slip (Trace 3).

In Plate 6.3 continuous slip persists despite the angle of inclination.

Plate 6.4 is for a horizontal conveyor with a heavy load being transported. Some relay chatter can be seen in the acceleration waveform which has discontinuities. The motion is double sided stick/slip.

Plate 6.5 is for a similar case with continuous slip.

Plate 6.6 shows the phase-plane trajectory of the most complicated motion of System A, denoted on Fig. 6.4 as $\frac{1}{4}(9)$. This notion is 
mentioned in Appendix $\mathrm{E}$ where it is considered to be a $\frac{17}{4}$ ultrasubharmonic rather than a $\frac{1}{4}$ subharmonic. This motion is very sensitive to small changes and took a long time to settle down.

Plate 6.7 shows that velocity, displacenent curves could also be obtained for the material this plate shows a $\frac{1}{2}$ subharmonic.

Plate 6.8 shows the effect of the jump in acceleration when the material is heavy. This phase-plane is for single sided slip, harmonic solution. Three cycles of material motion are shown in the lower picture.

Fig. 6.38 shows the phase-plane of a harmonic solution in which an intersection occurs. The samp waveform is plotted in 3-dimensions in Fig. 6.39 to give a 'phase-space' and from the projection in the $X-t$.plane using the periodic time representation sin $t$.it can be seen that the intersection of curves no longer takes place.

Fig. 6.40 illustrates that the results obtained from the X-Y plotter can be plotted from the results of the digital computer programme. 6.5 Discussion of the relative mertis of solution by analogue and digital

The agreement between solutions obtained by the two computers is good throughout all the results taken, the only disagreement being in the complicated regions of solution of Figs. 6.4 - 6.6. In other cases, estimates of the uegree of agreement is assessed as being between 5 10\%, though occasionally higher when travel times were large. It would thus appear that both solutions are equally acceptable in terms of accuracy

The type of non-linearity involved made solution by analogue computer particularly convenient for this problem. The degree of accuracy which can be obtained depends on the size and accuracy of the machine and the 
skill of the user. There were several known sources of error in the analogue computer circuit, most of which have been mentioned before. Despite slowing down the problem to a very slow speed, some time lag was inevitable when several relays were involved in one circuit. Relay chatter was another difficulty experienced sometines brought about by the need to use very small signals to operate them, as, for example when $|\ddot{x}|$ was very slightly greater than $\mu \mathrm{g}$. Even had all other parts of the circuit been perfect the means of obtaining the relative velocity by the use of two integrators and a summer is not exact. The initial condition voltage would always have a time lag compared with the input signal, so that in sinusoidal motion with no inclination an error was found when large signals were involved, giving a small travel rate. This was unavoidable, and allowed for where possible. Otherwise the circuit worked well, the only insuperable problem being that the machines available were too small so deal with the full problem. The flexibility of the analogue computer in investigating a range of variables would make it a most valuable tool in designing a working conveyor.

The digital computer progranme is not without interest. It could be developed almost indefinitely to include any complexity if machine time and money were of no importance. Several approximation were used in this solution, though none were important or contained much inaccuracy. The damping constant was not, in genoral, changed for ecish region or sulution, though this was done in one programme and was found to have negligible effect. While relative displacements were calculated by Simpson's Rule over 100 ordinates per cycle, and trapezoidal rule was used to calculate the slider displacenent when a vertical acceleration was present, and this may have produced a small error. Finally, linear interpolation was used to establish the instant at which changes in regions occurred, 
but the error in $1 / 100$ of a cycle could not be significant, and was not cumulative. This was checked using a Newton-Rapheson iteration, which used more machine time and gave no change in waveform: Obviously some limit must be put on a digital progranne, but accuracy to six or more decimal places is available if required, and machine time is of no consequence. The information required from the digital computer must be pre-determined, and in this it is less flexible than the analogue computer. However the results obtained agreed with those from the analogue machine and with such of those of Booth and McCallion (4). as were comparable. 


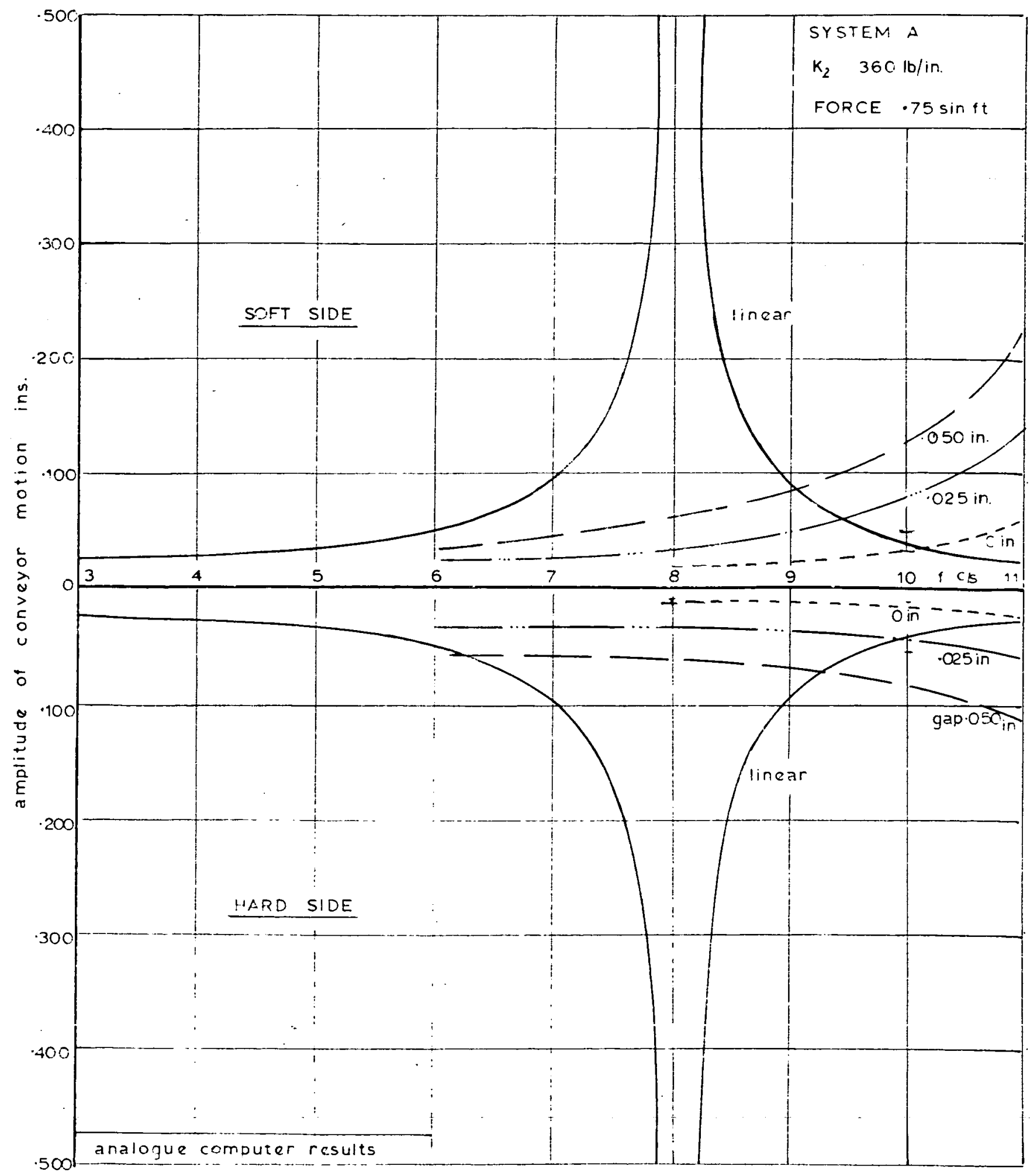

AMPLITUDE RESPONSE CURVES FOR SYSTEM A. FIG 6:1 


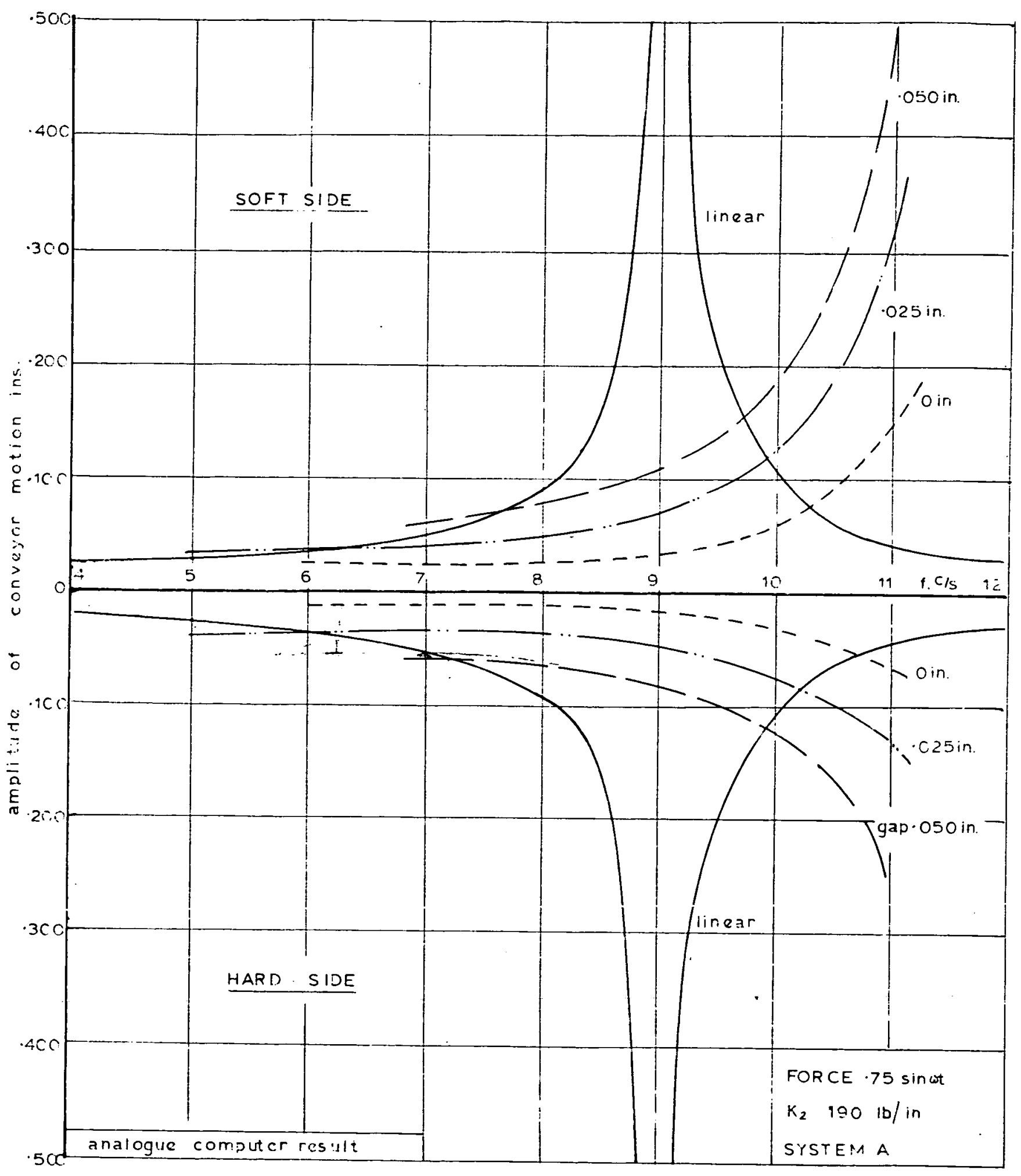

AMPLITUDE RESPONSE CURVES FOA SYSTEM A FIG $6: 2$ 


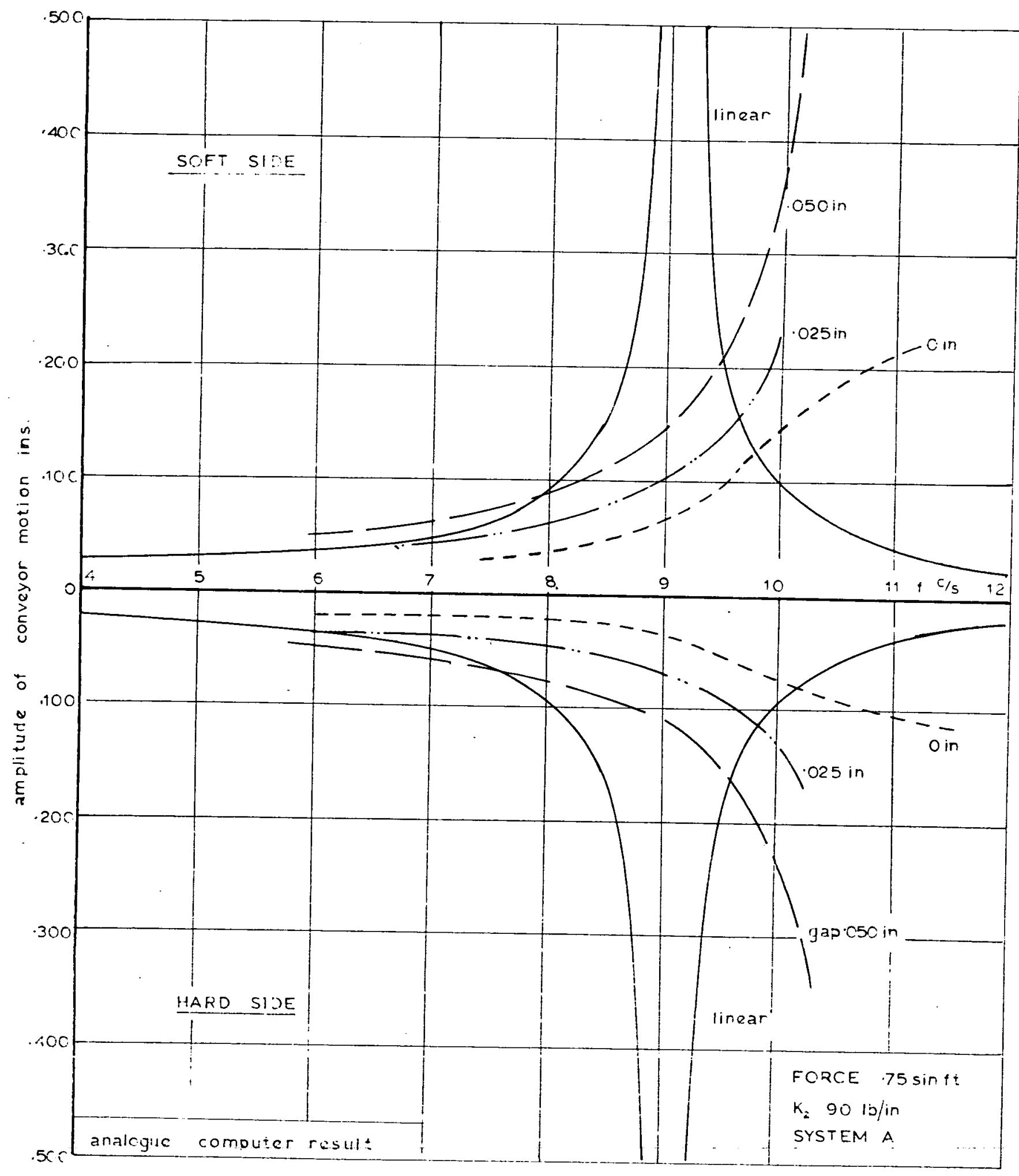

AMPLITUDE RESPONSE CURVES FOR, SYSTEM A

FIG.6:3 


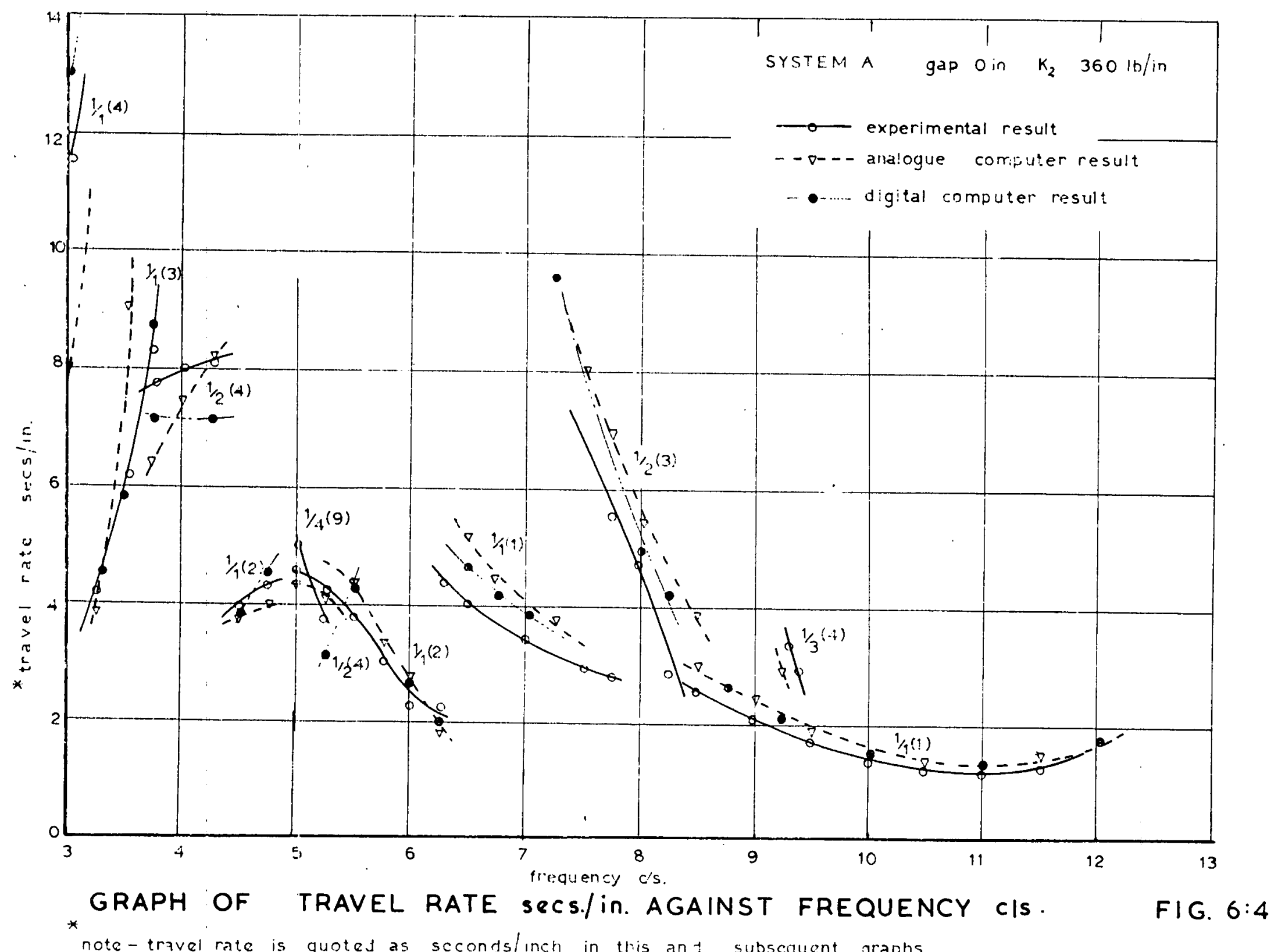




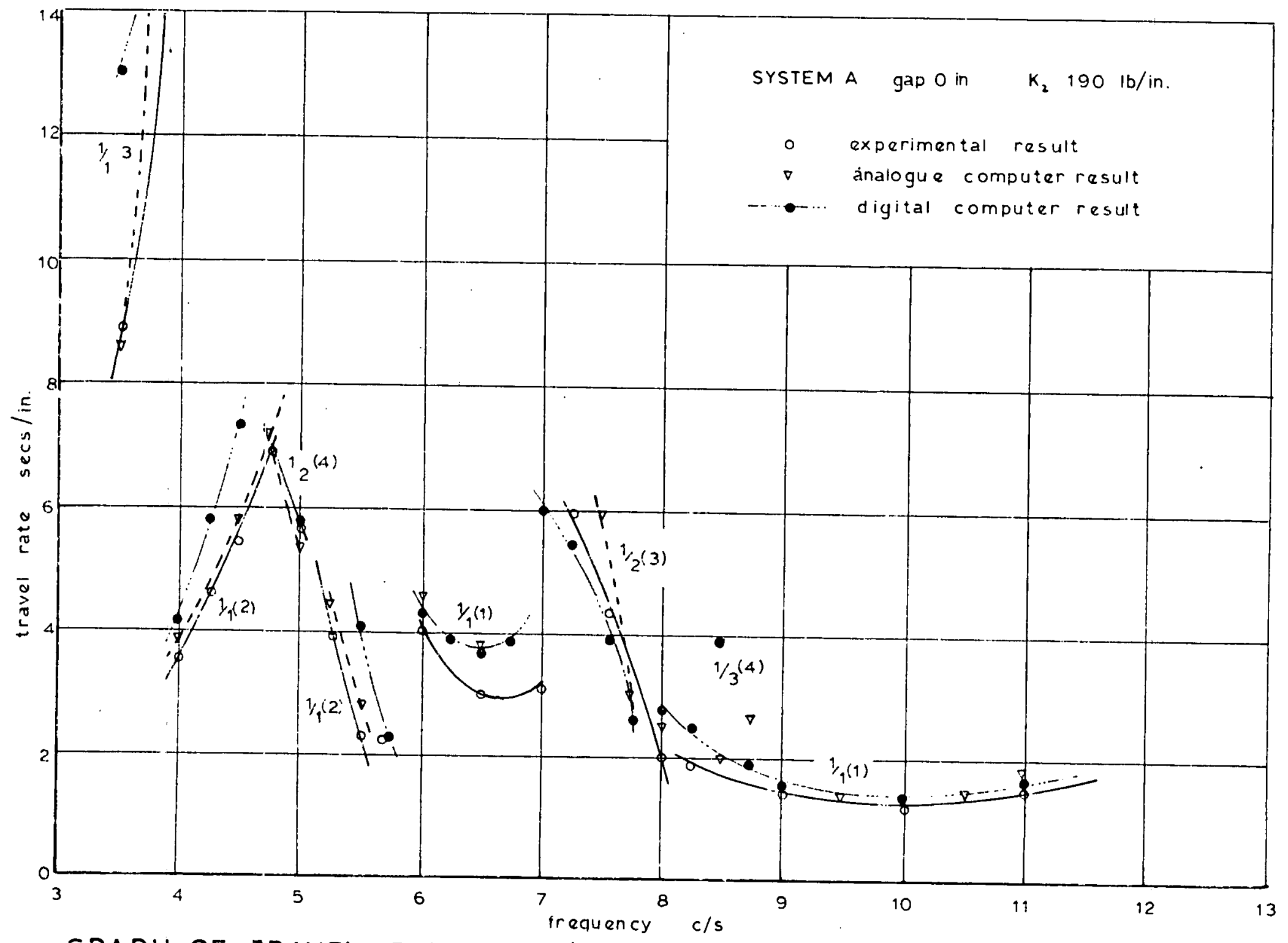

GRAPH OF TRAVEL RATE secs/in. AGAINST FREQUENCY c/s.

FIG. $6: 5$ 


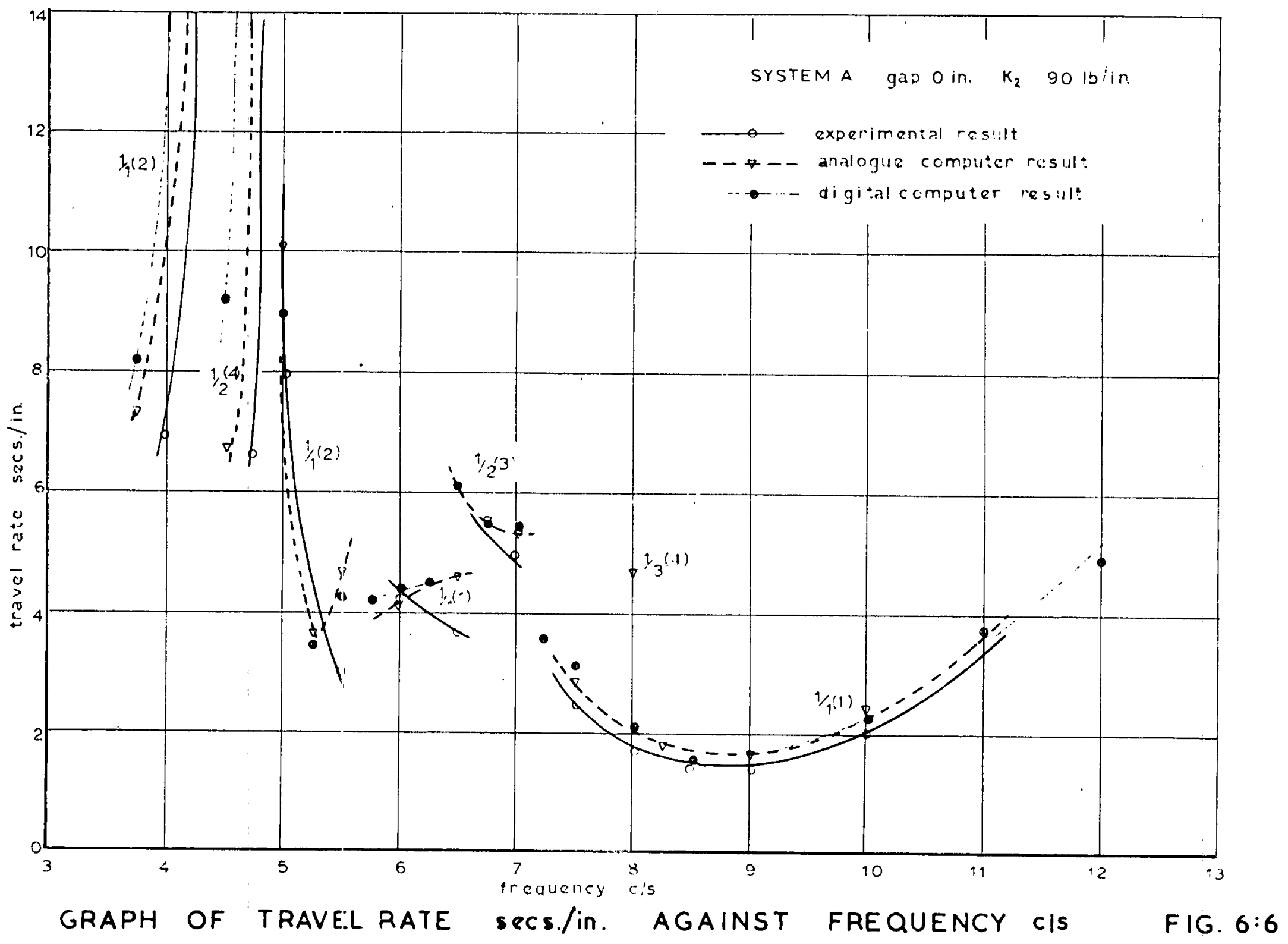




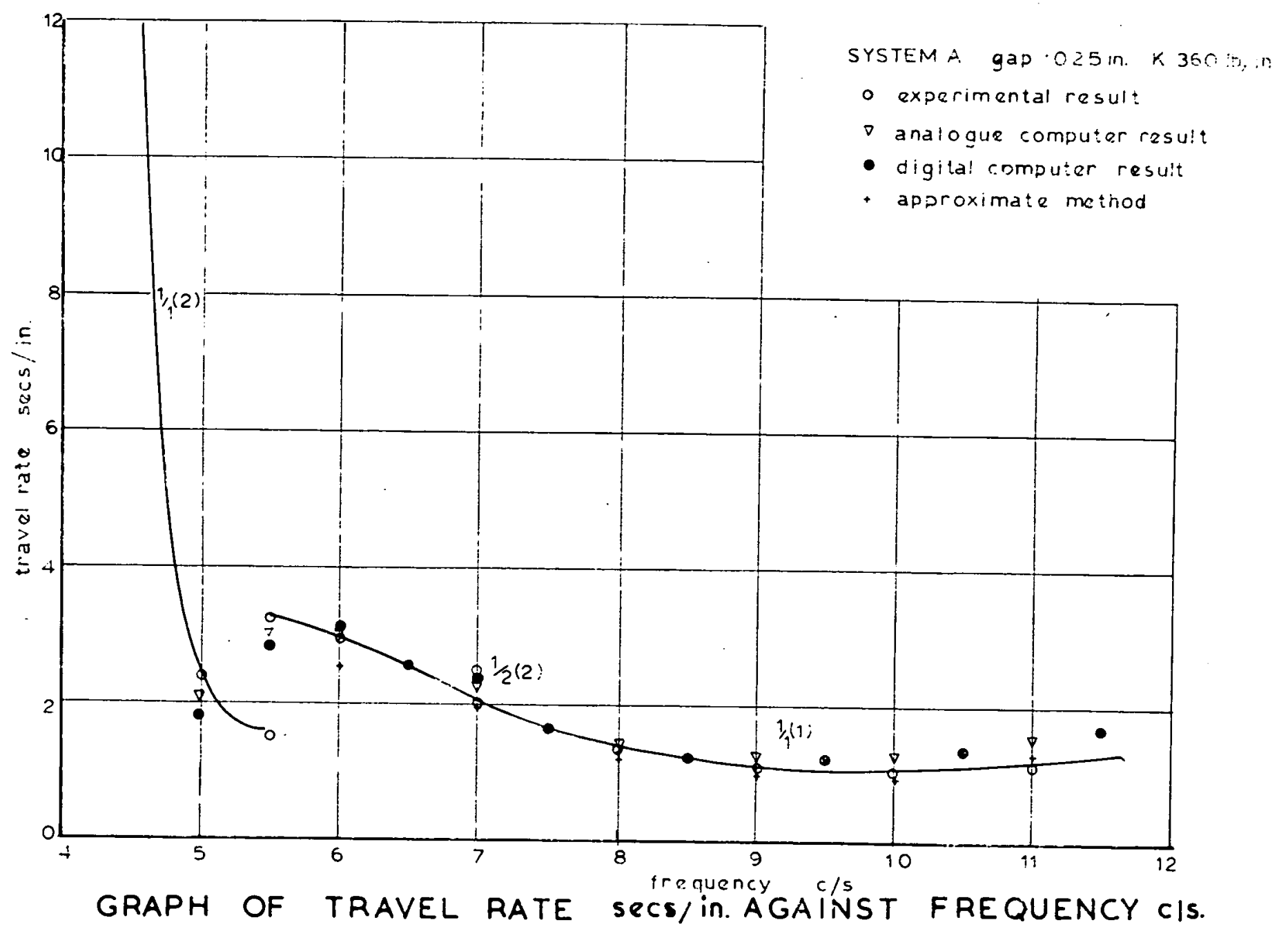

FIG. $6: 7$ 

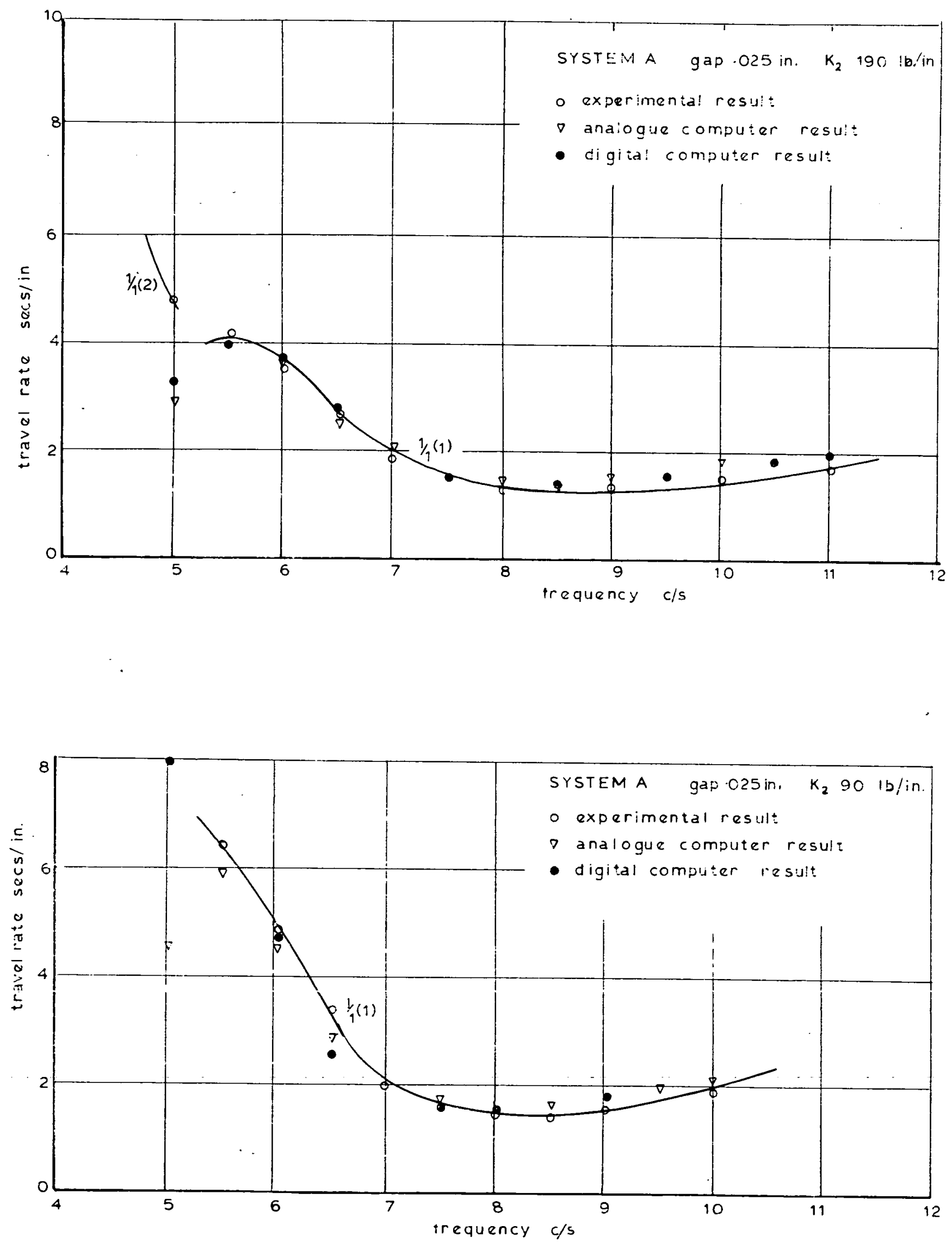

GRAPHS OF TRAVEL RATE secs/in. AGAINST FREQUENCY cls

FIG: $6: 8$ 


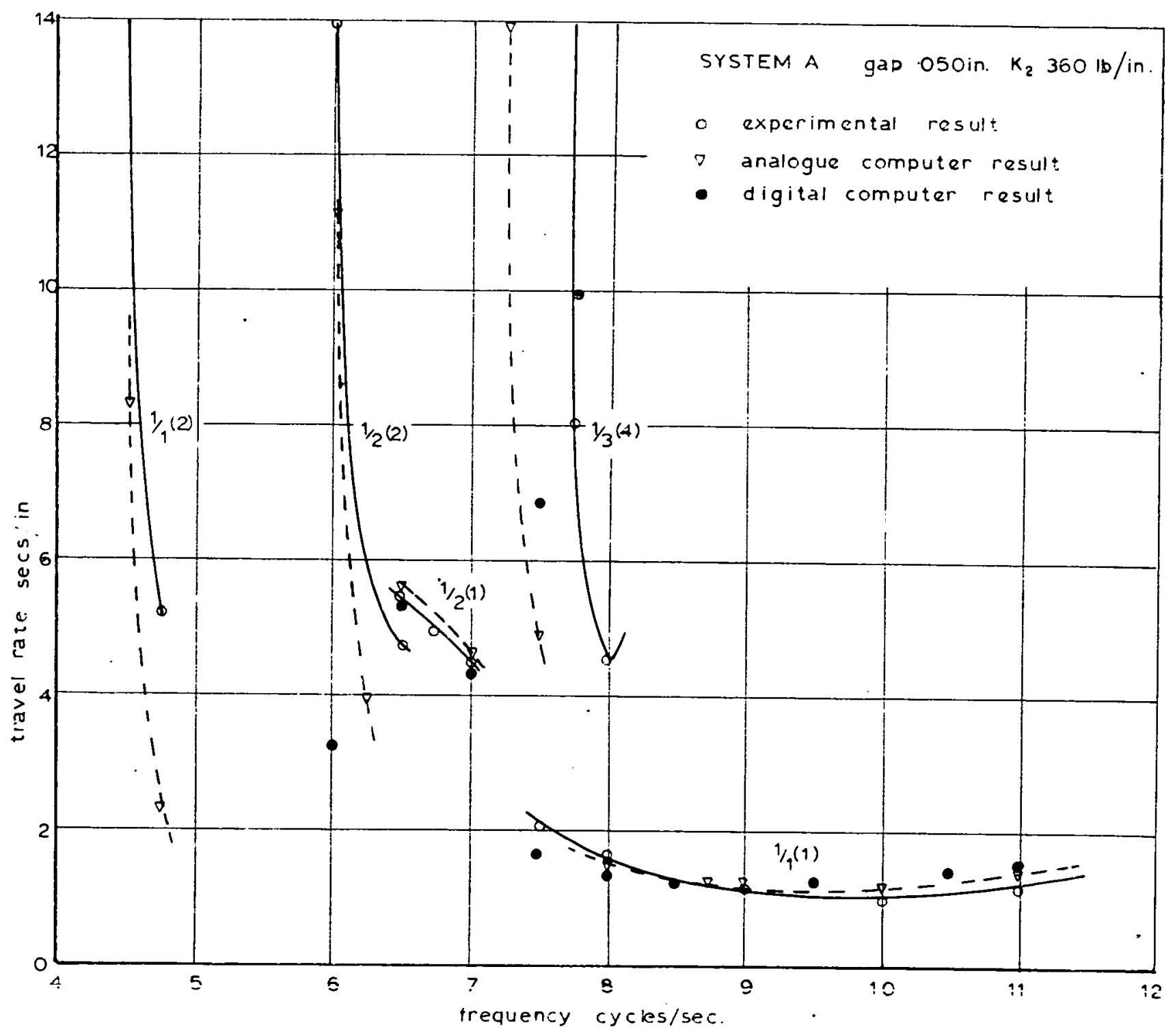

GRAPH OF TRAVEL RATE secs/in AGAINST FREQUENCY CIS.

FIG. 6:9 

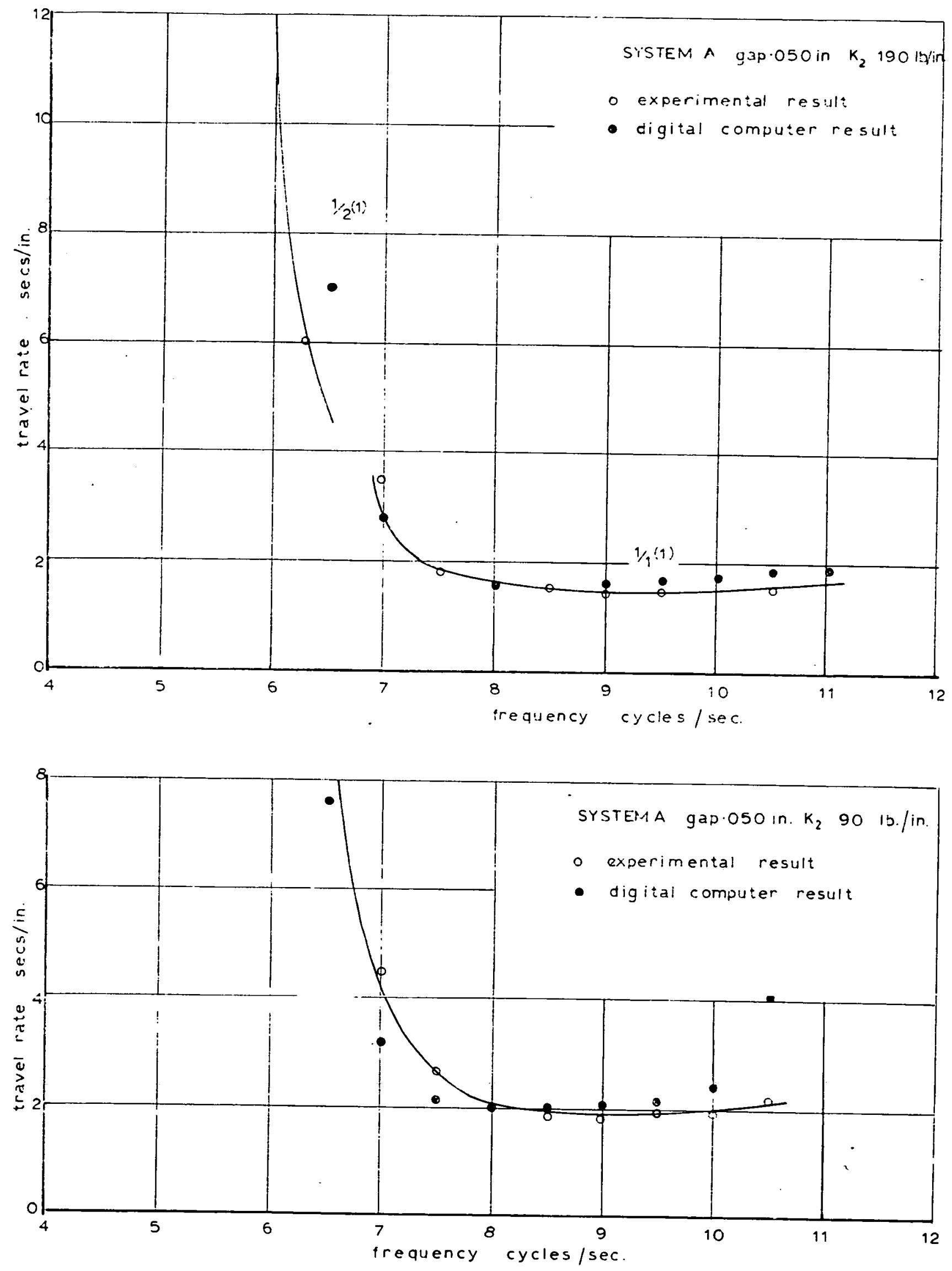

GRAPHS OF TRAVEL RATE secs/in AGAINST FREQUENCY $\mathrm{cls}$

FIG. $6: 10$ 


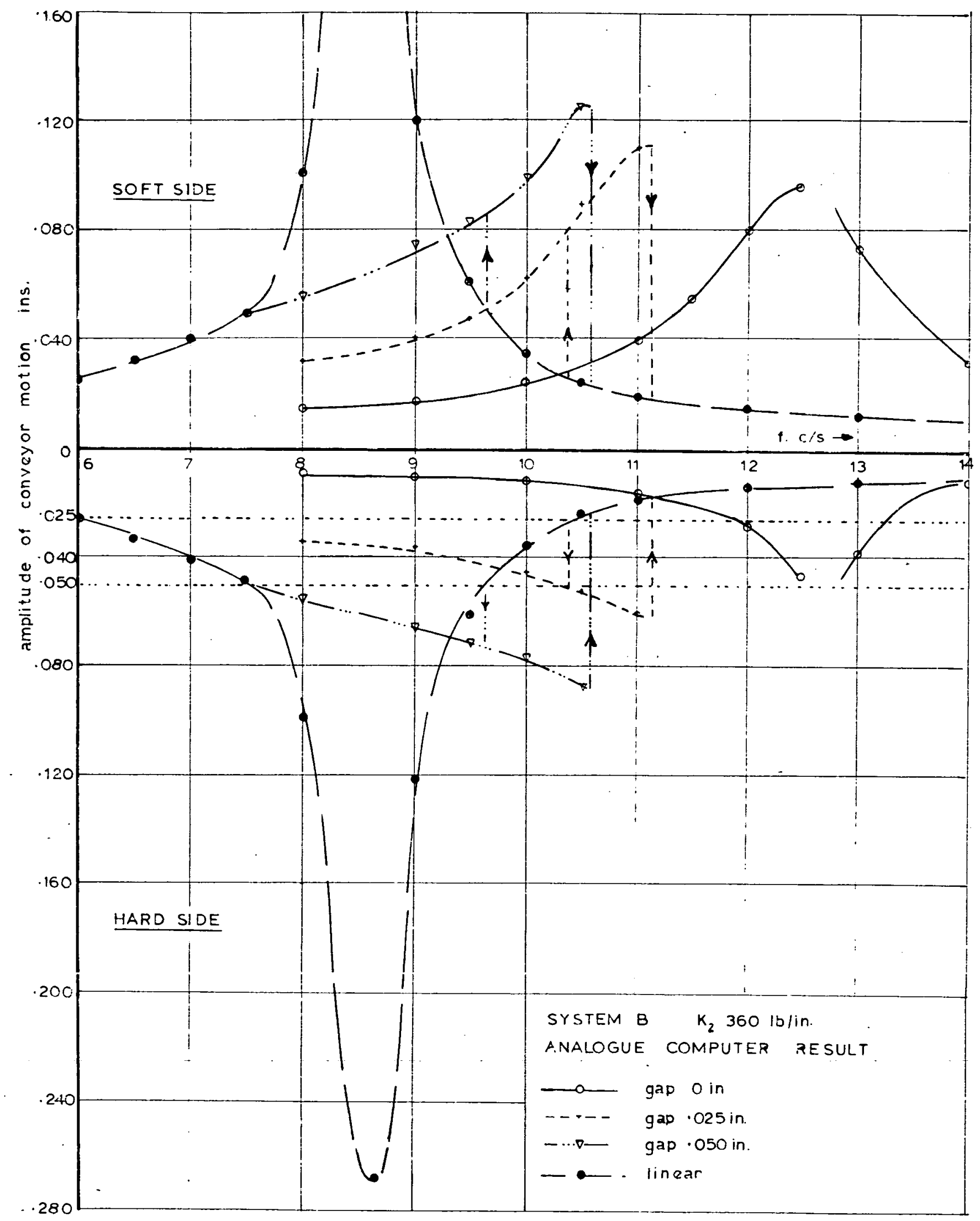

AMPLITUDE OF CONVEYOR MOTION ins. AGAINST FREQUENCY cls.

FIG. 6:11 


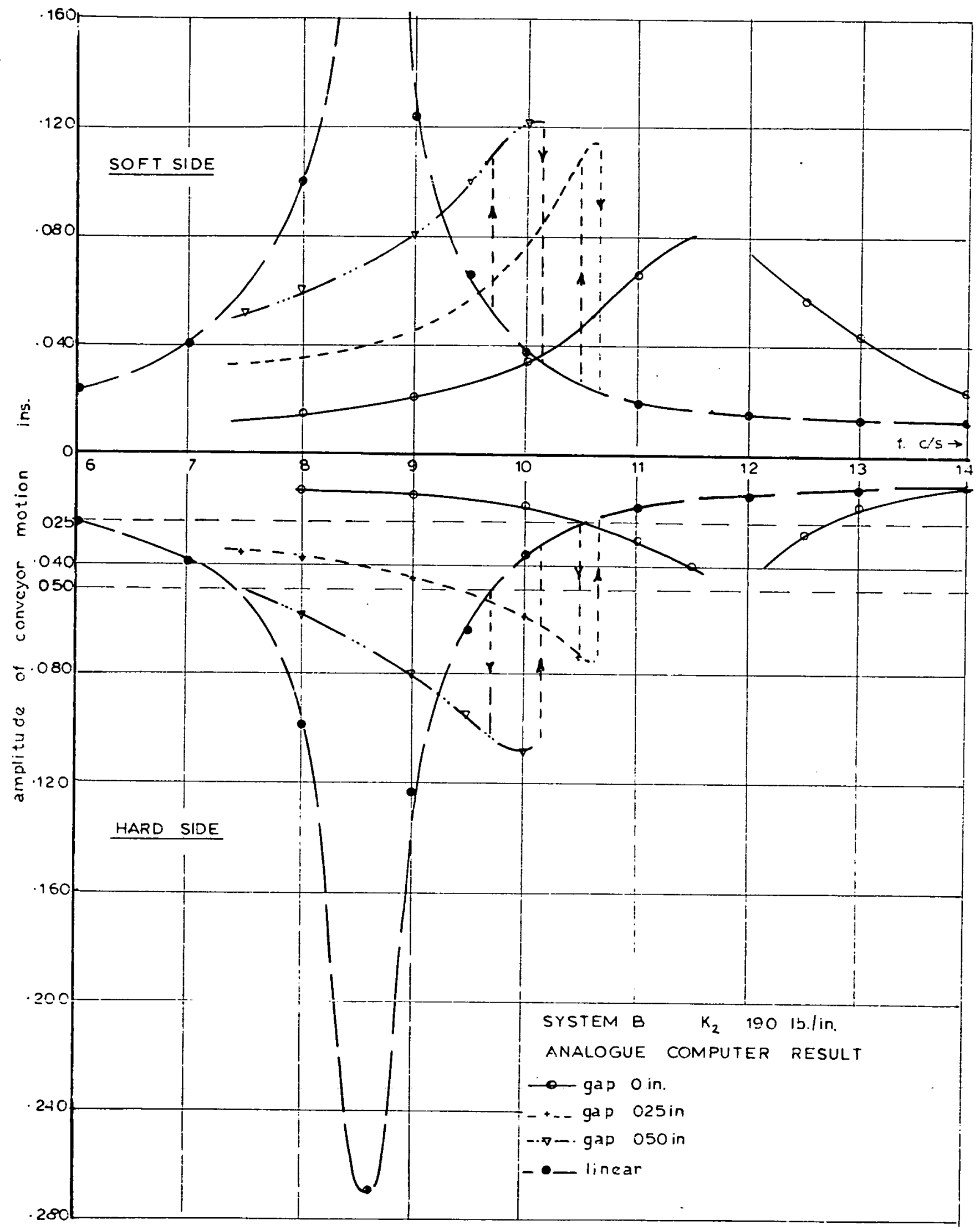

AMPLITUDE OF CONVEYOR MOTION ins. AGAINST FREQUENCY cls.

FIG. $6: 12$ 


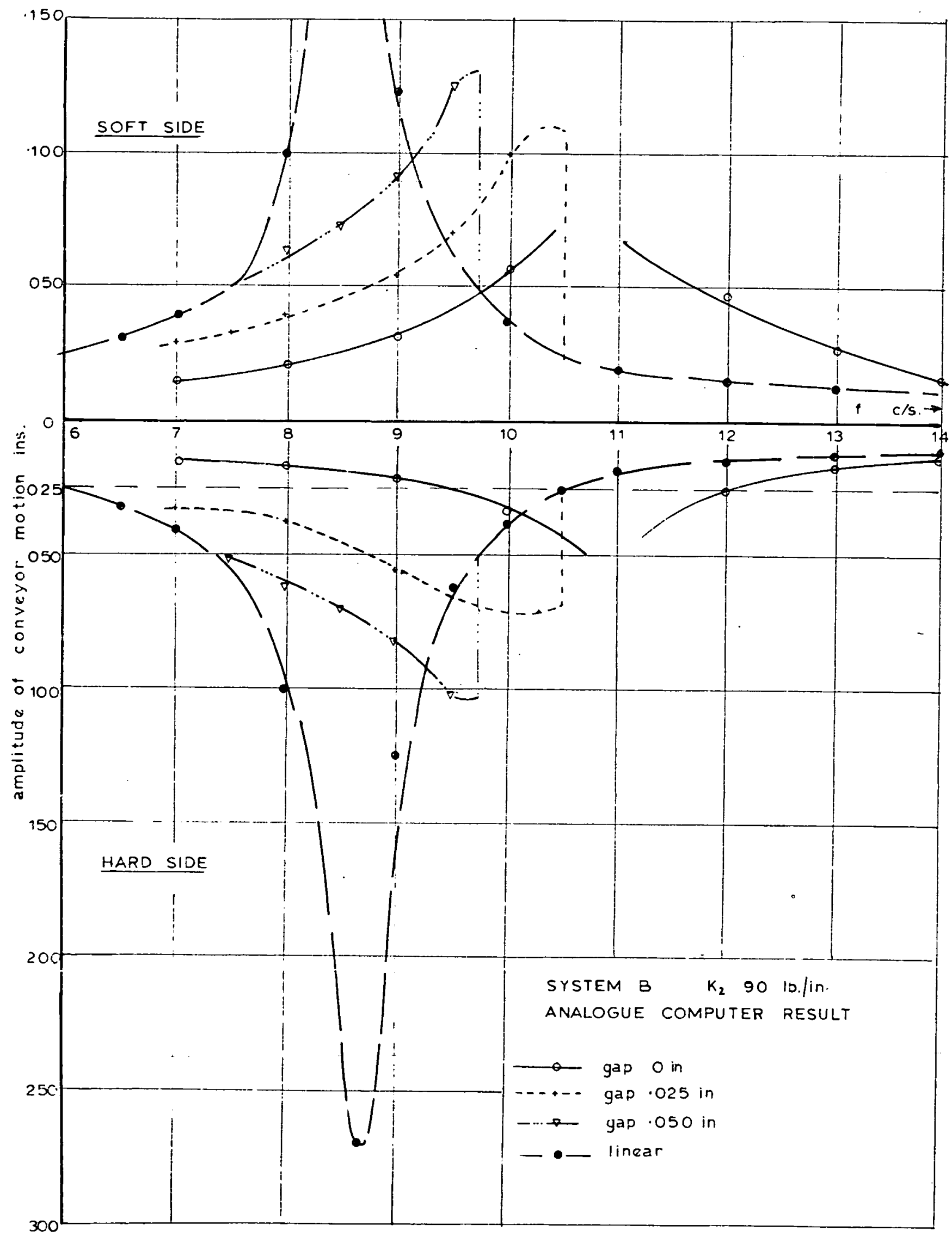

AMPLITUDE OF CONVEYOR MOTION ins. AGAINST FREQUENCY CIS.

FIG. $6: 13$ 


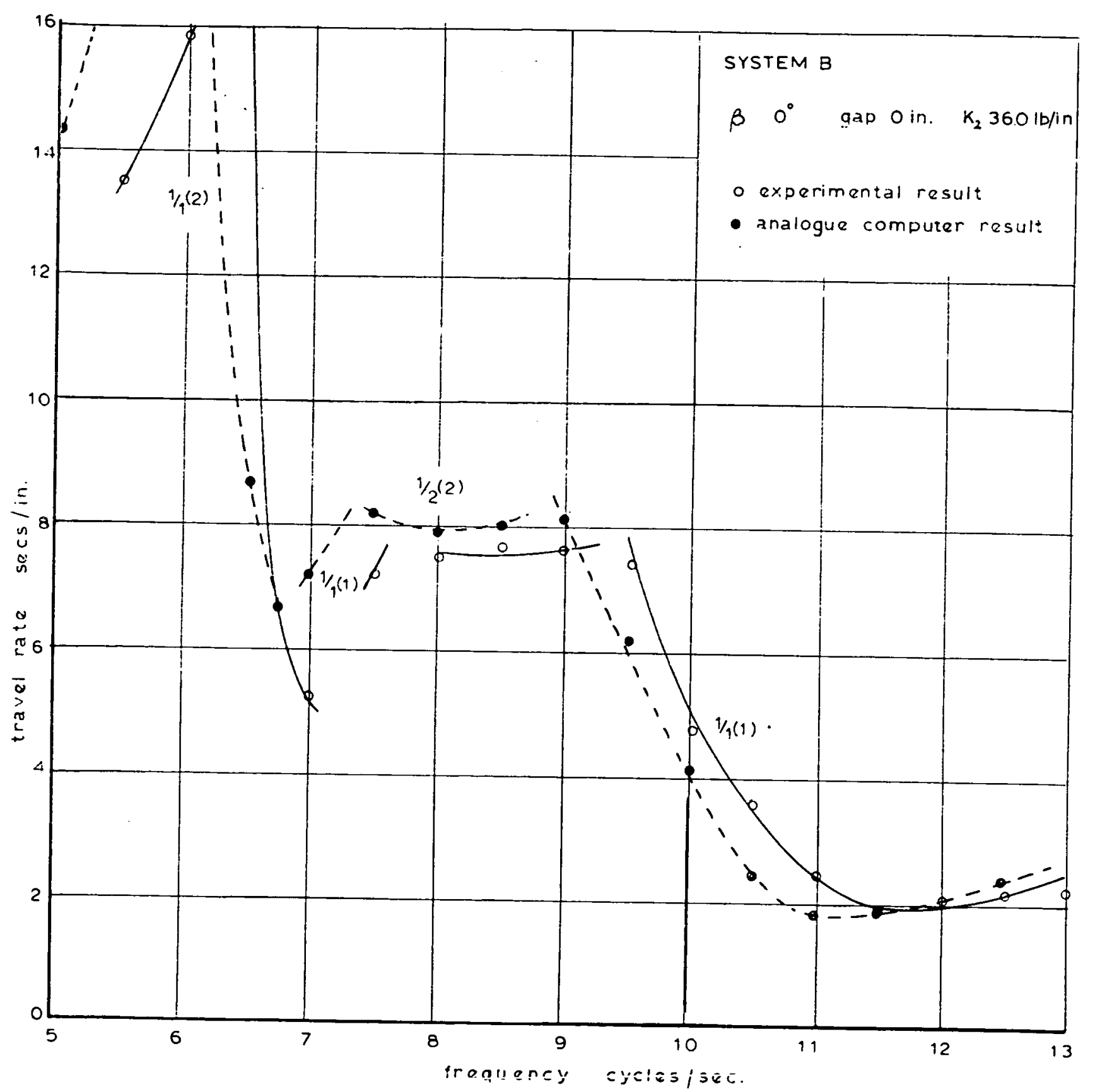

EXPERIMENTAL RESULTS \& ANALOGUE COMPUTER RESULTS GRAPH OF TRAVEL RATE secs/in AGAINST FREQUENCY cls

FIG. 6:14 

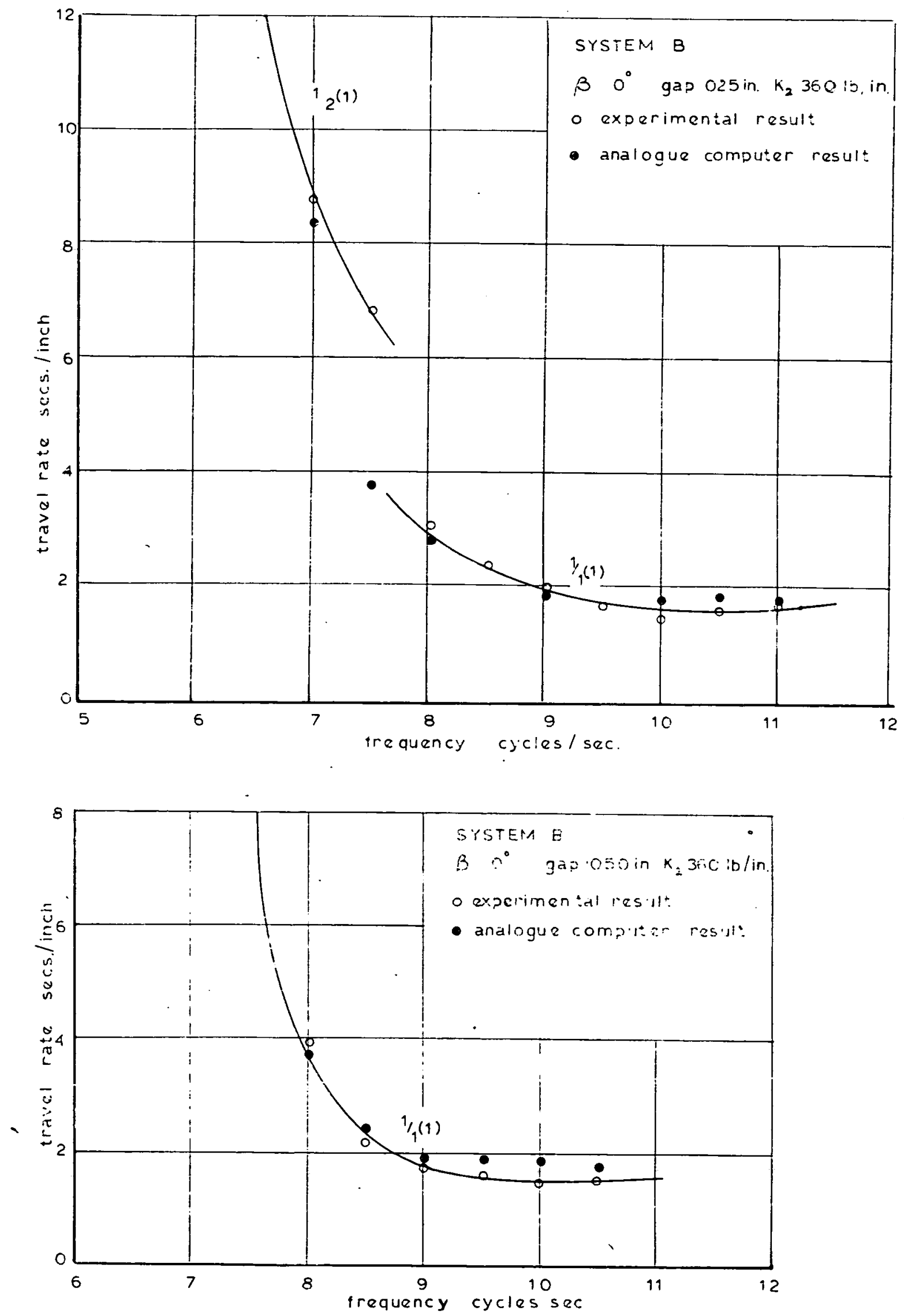

EXPERIMENTAL \& ANALOGUE COMPUTER RESULTS. GRAPH OF TRAVEL RATE sECS/in. AGAINST FREQUENCY cls.

FIG. 6:15 


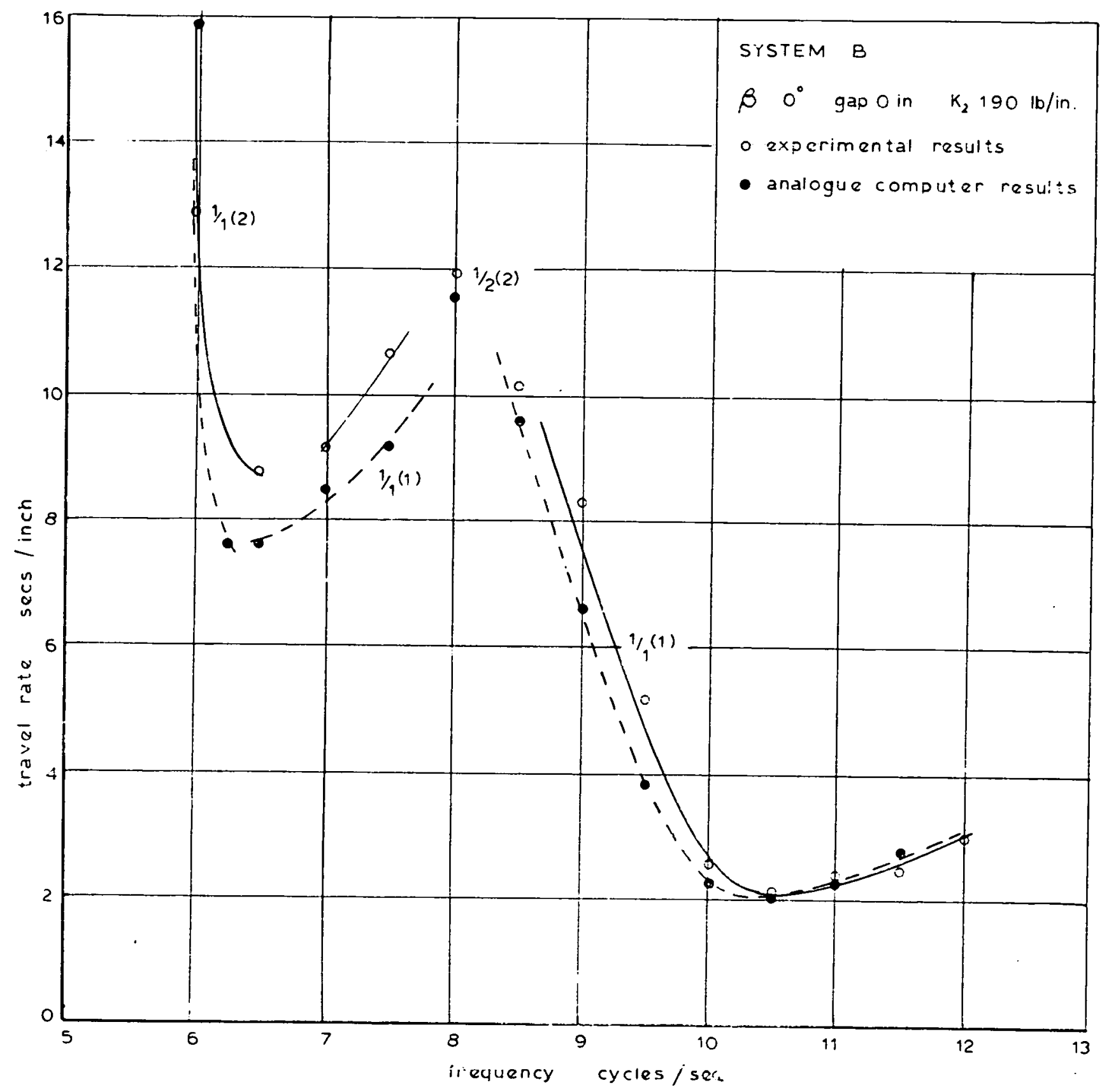

EXPERIMENTAL \& ANALOGUE COMPUTER RESULTS GRAPH OF TRAVEL RATE secs/in. AGAINST FREQUENCY cls FIG. 6:16 

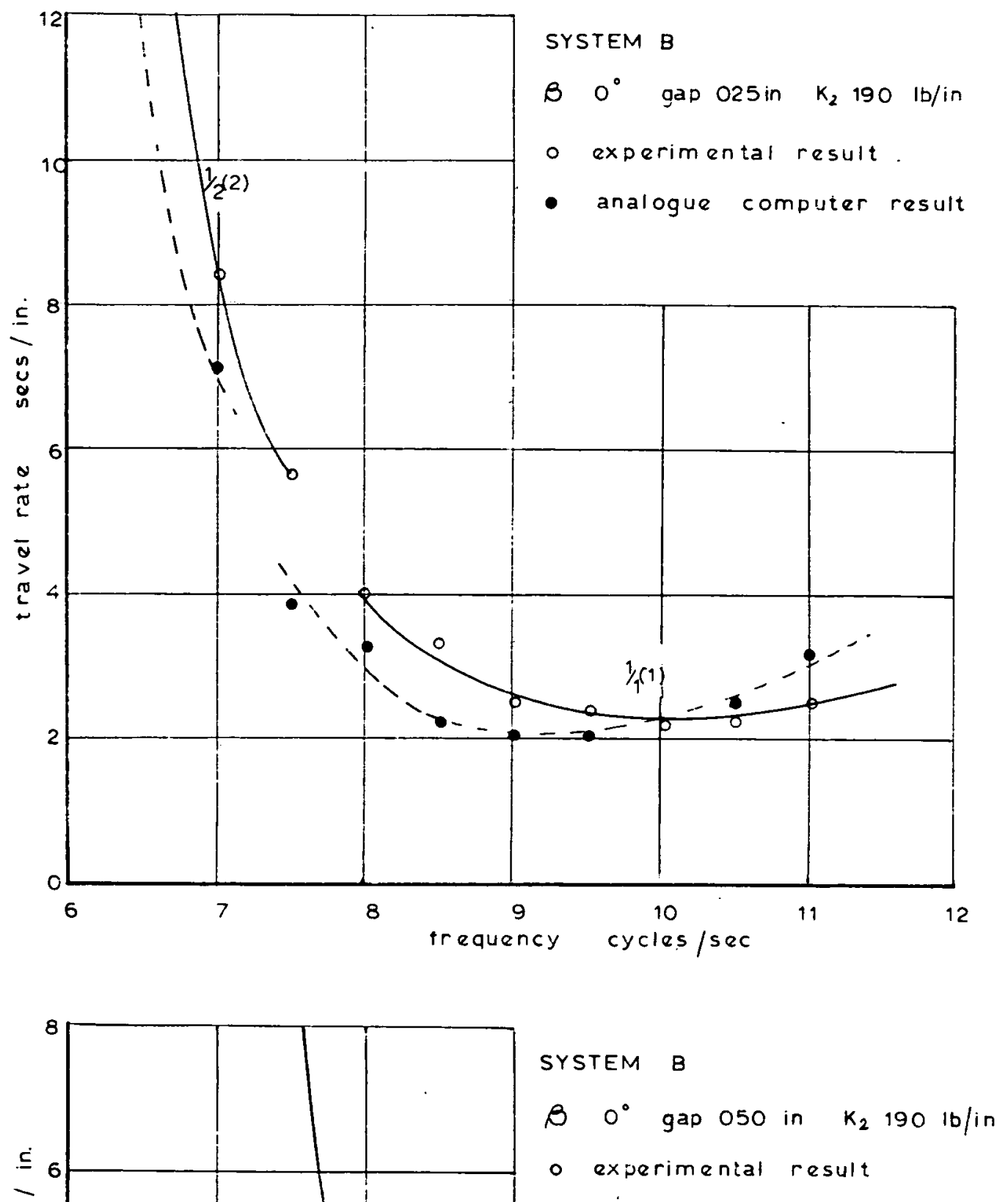

- analogue computer result

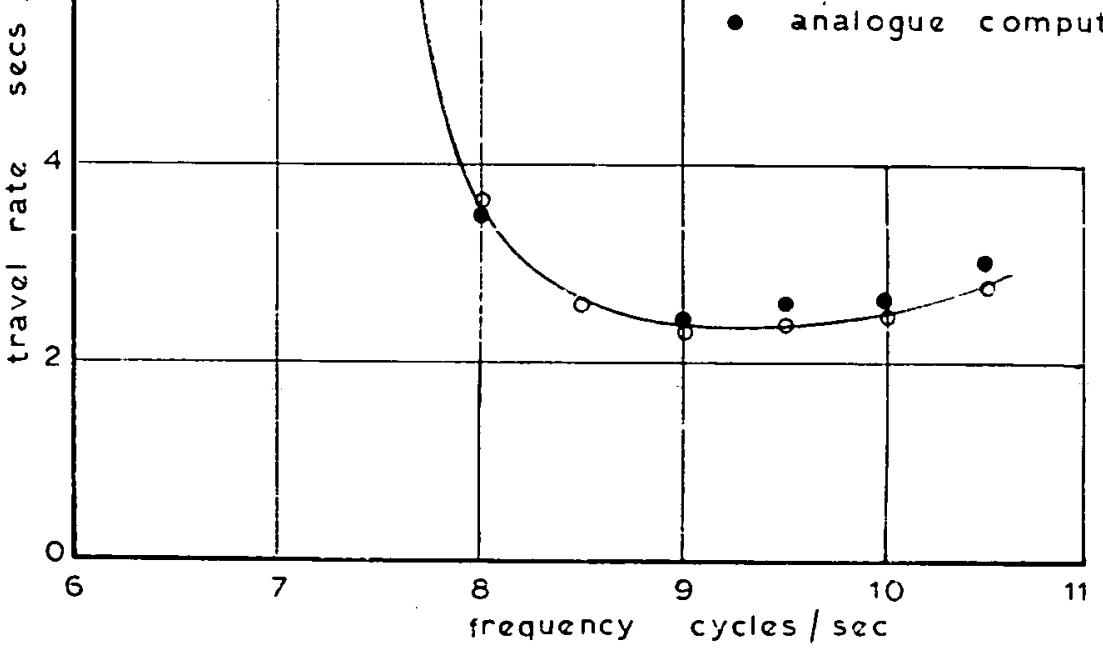

EXPERIMENTAL \& ANALOGUE COMPUTER RESULTS GRAPHS OF TRAVEL RATE seCs/in. AGAINST FREQUENCY CIs.

FIG. $6: 17$ 


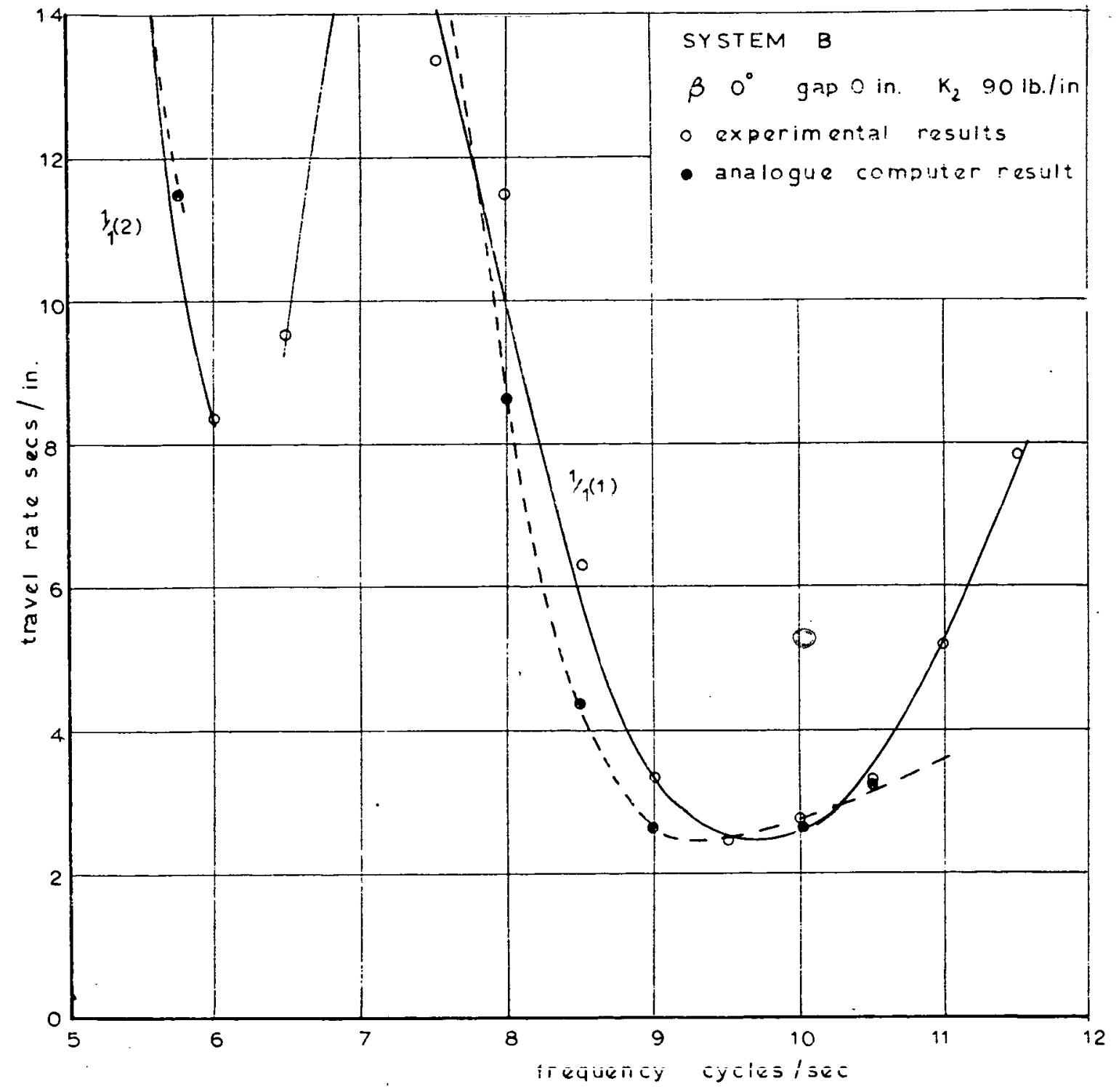

GRAPH OF TRAVEL RATE sPCs/in AGAINST FREQUENCY cIS

FIG. $6: 18$ 

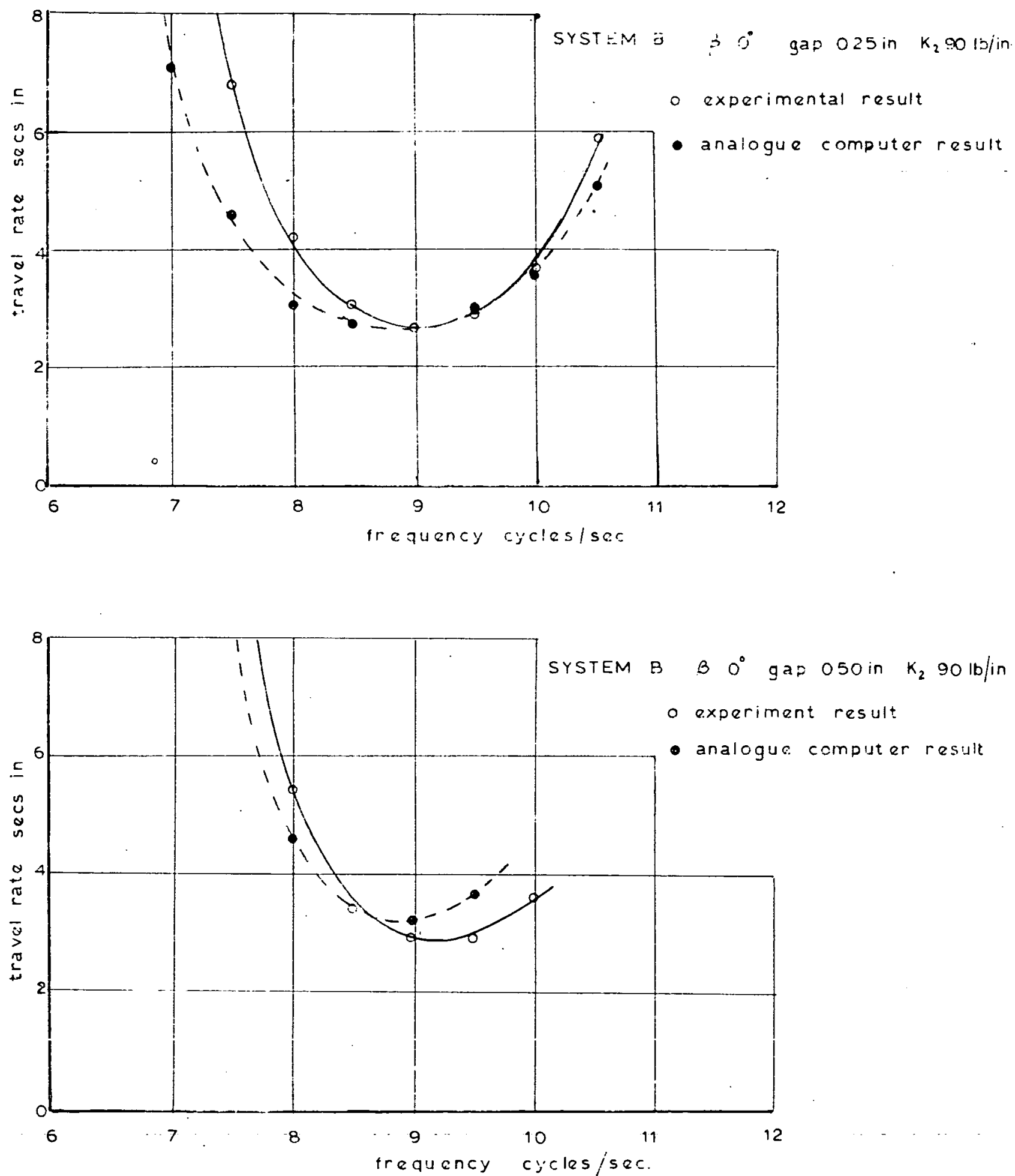

GRAPH OF TRAVEL RATE secs/in AGAINST FREQUENCY c/s

FIG $6: 19$ 


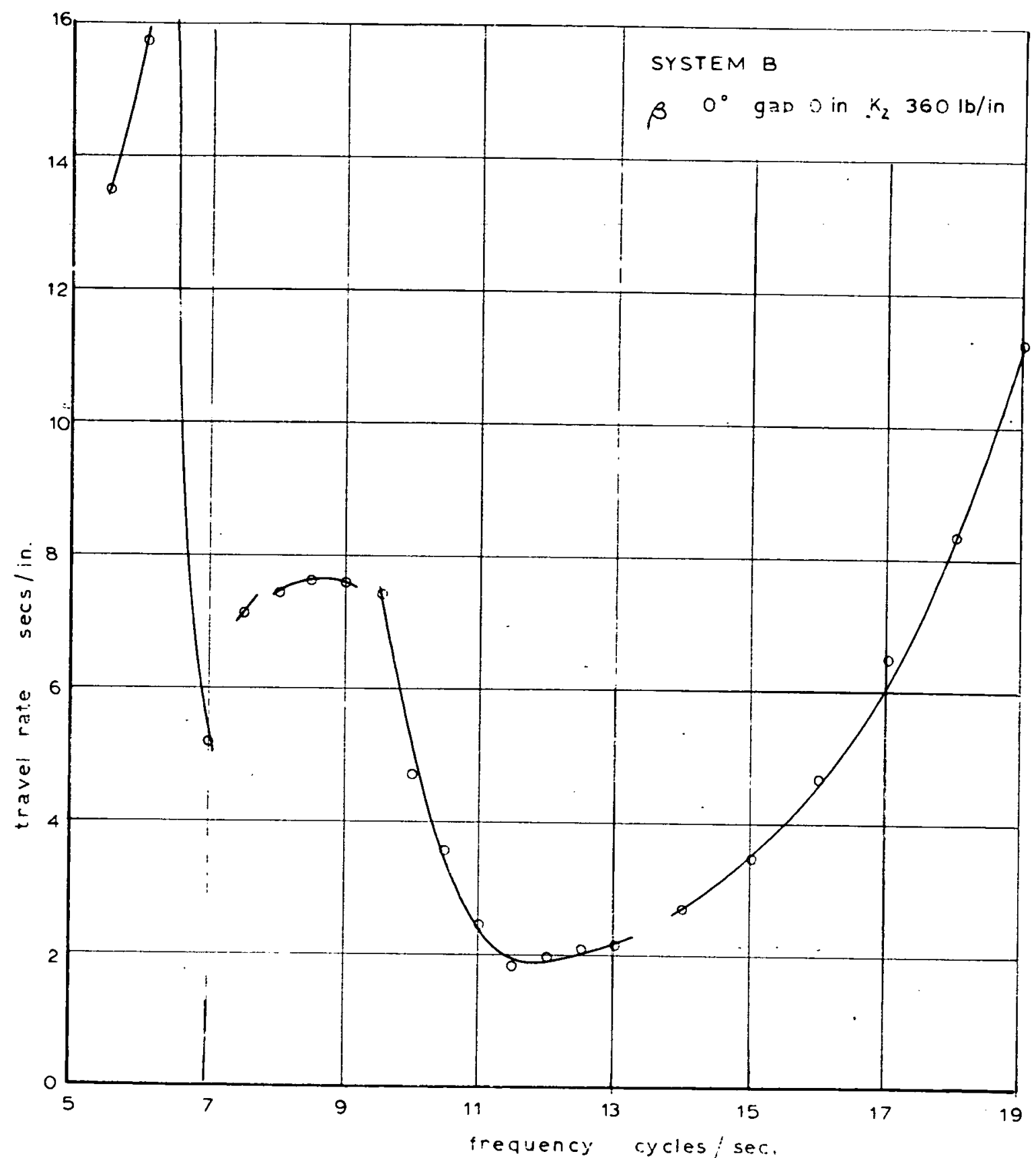

EXPERIMENTAL RESULTS SHOWING TRAVEL RATE secs/in. AGAINST TREQUENCY CIS. OVER RESONANCE JUMP

FIG. 6:20 


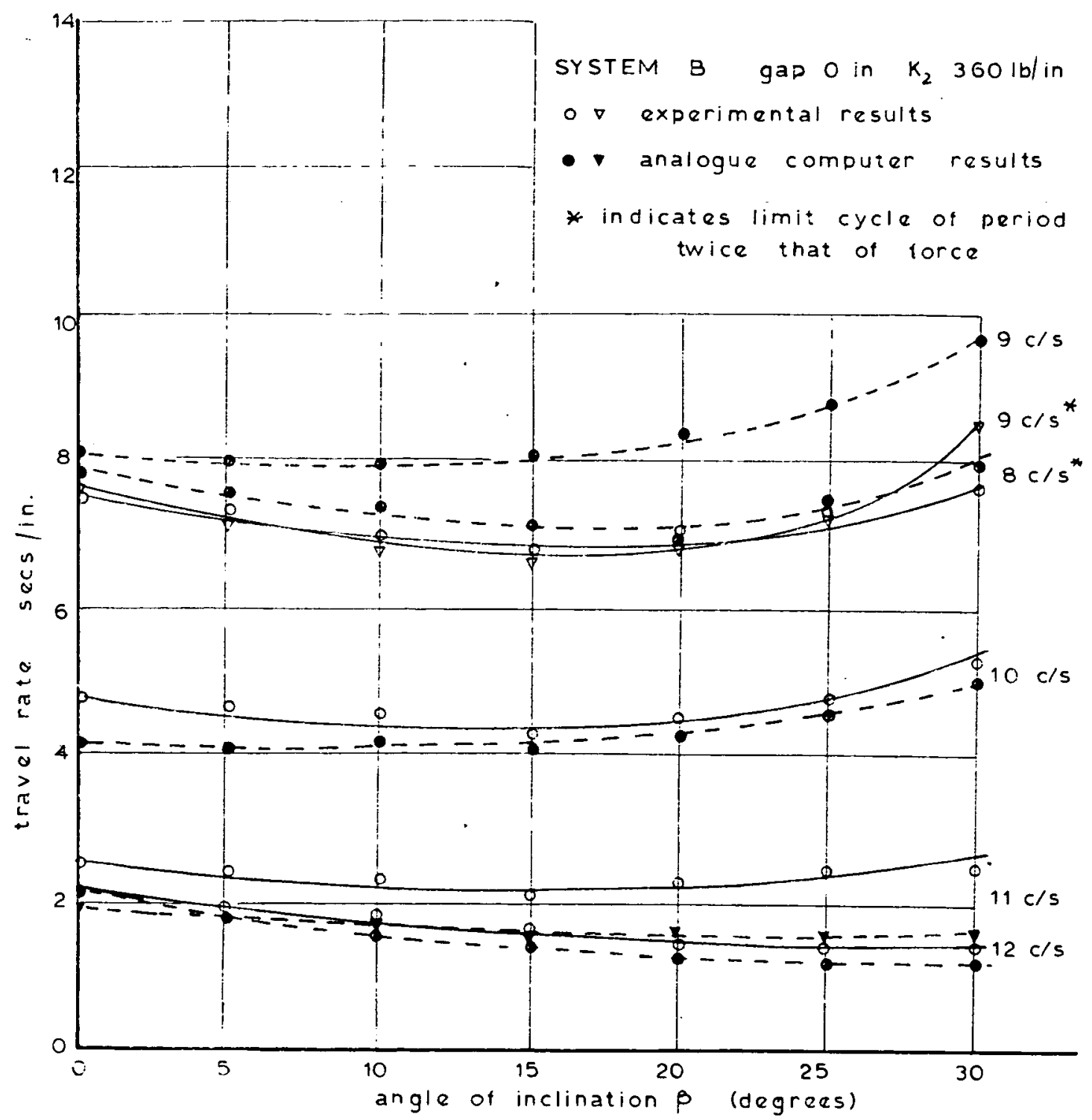

GRAPH OF TRAVEL RATE SECS/IN AGAINST ANGLE OF INCLINATION OF CONVEYOR VIBRATION $B^{\circ}$

FIG. $6: 21$ 

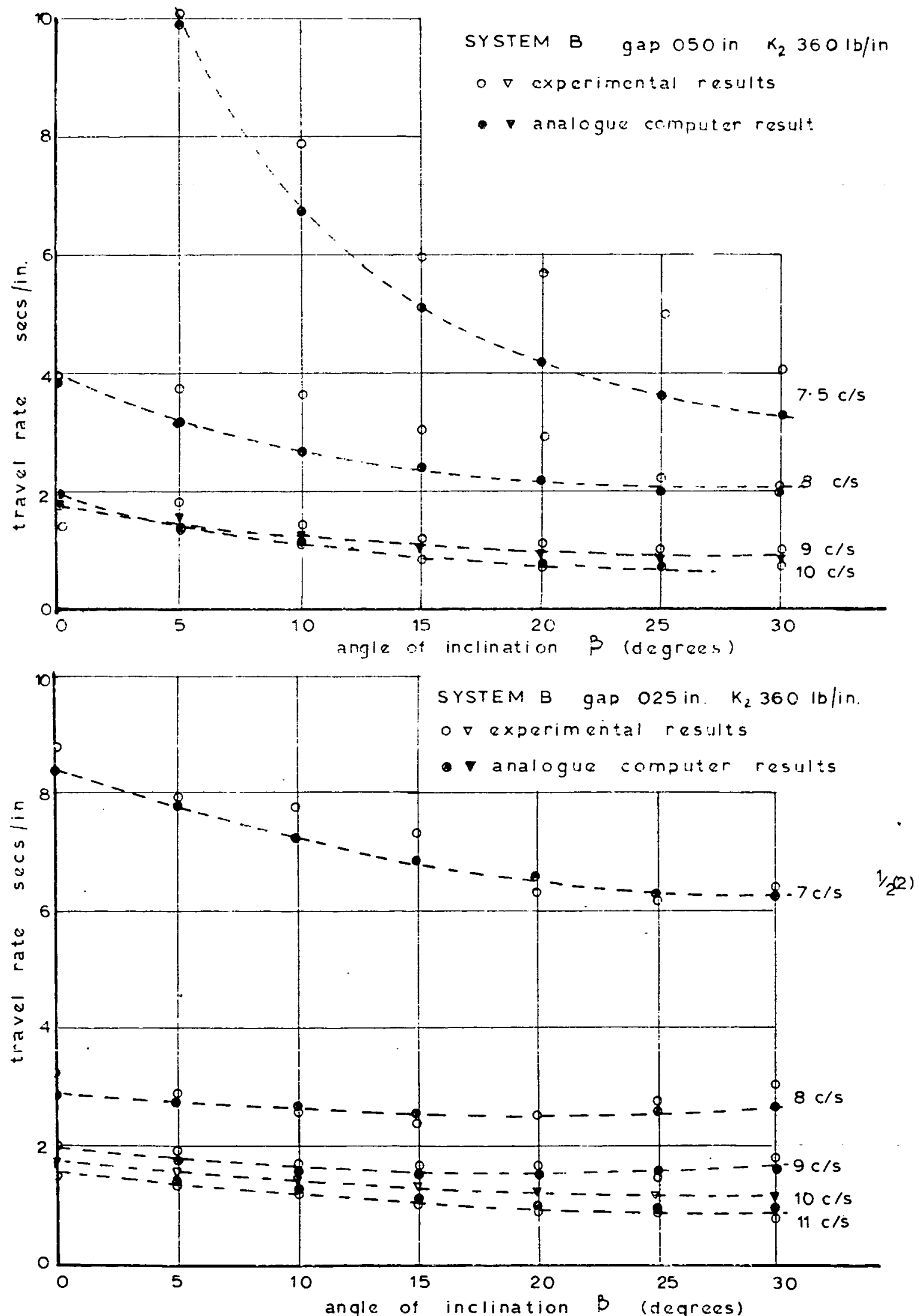

GRAPH OF TRAVEL RATE secs/in. AGAINST ANGLE OF INCLINATION OF CONVEYOR VIBRATION $\beta^{\circ}$

FIG. $6: 22$ 


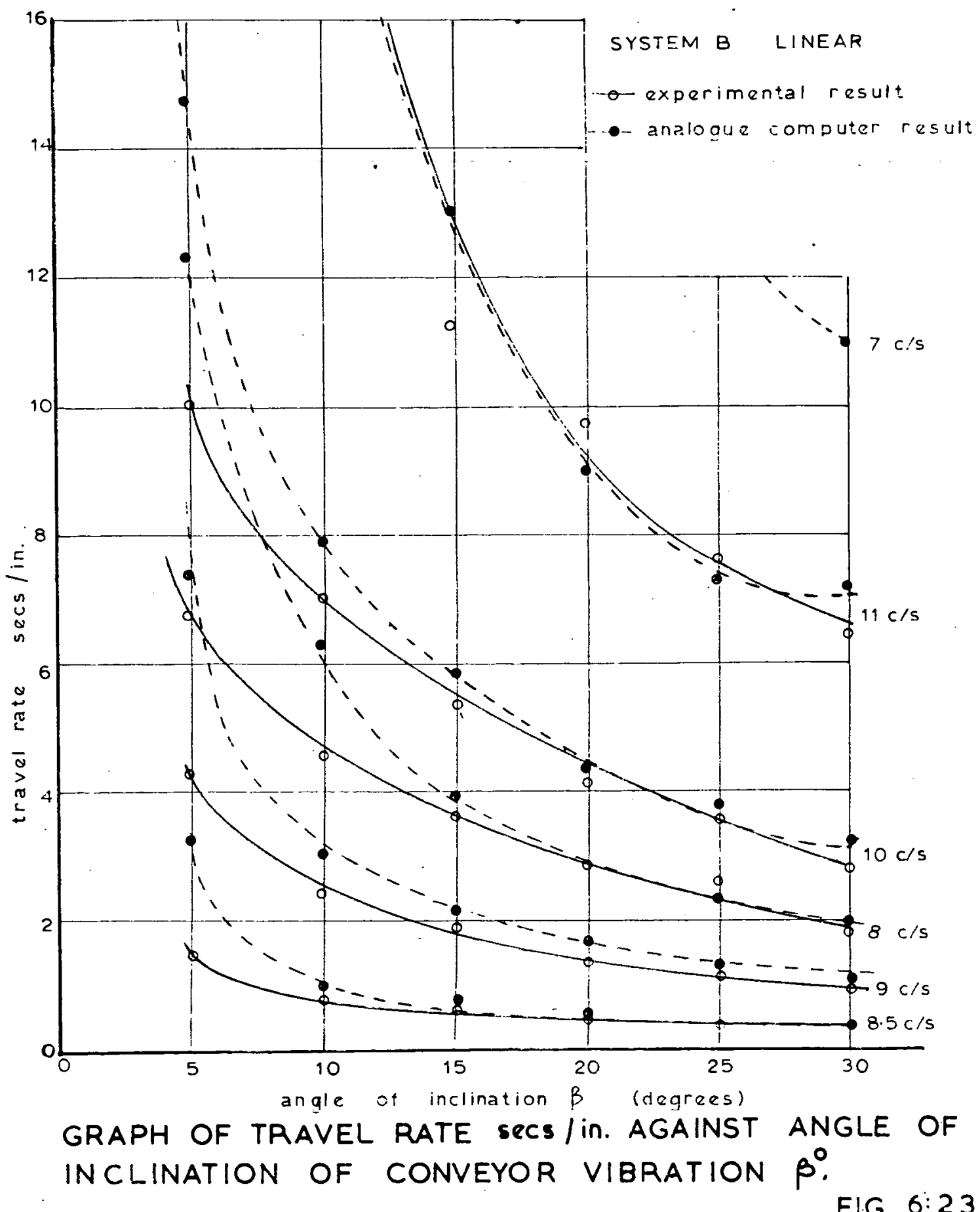




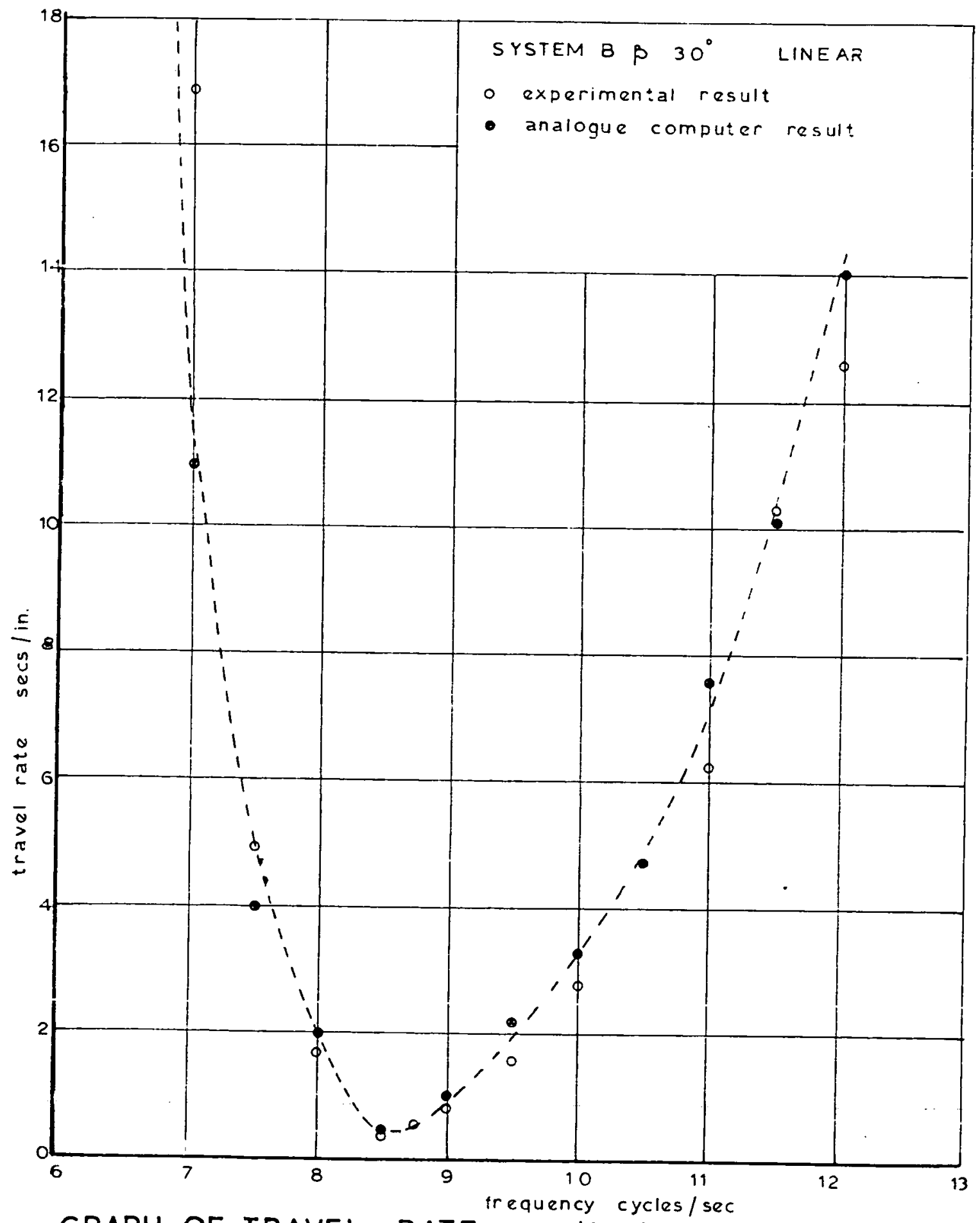

GRAPH OF TRAVEL RATE secs/in, AGAINST FREQUENCY cls.

FIG. $6: 24$ 


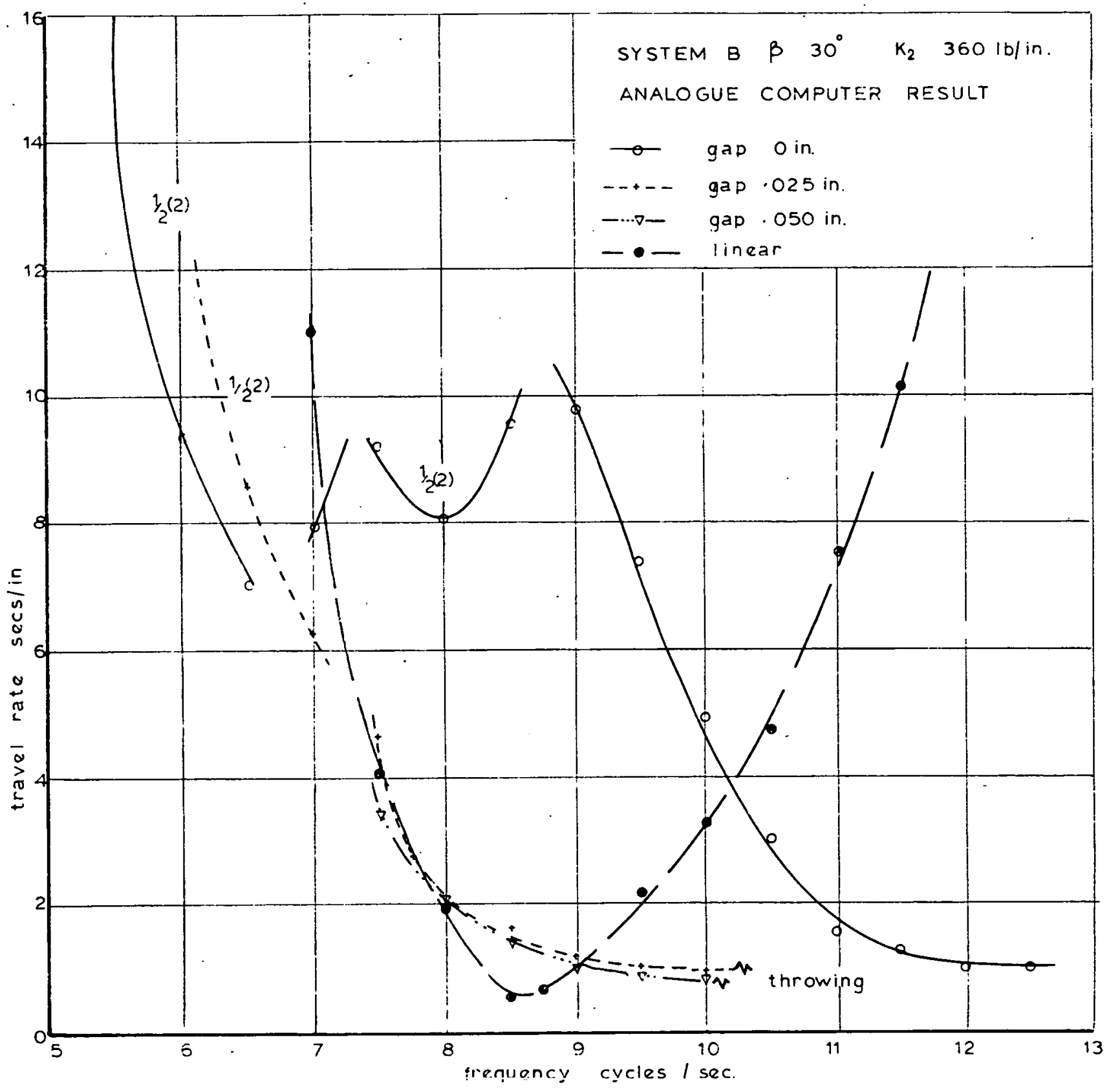

GRAPH OF TRAVEL RATE secs / in, AGAINST FREQUENCY cls.

FIG. $6: 25$ 


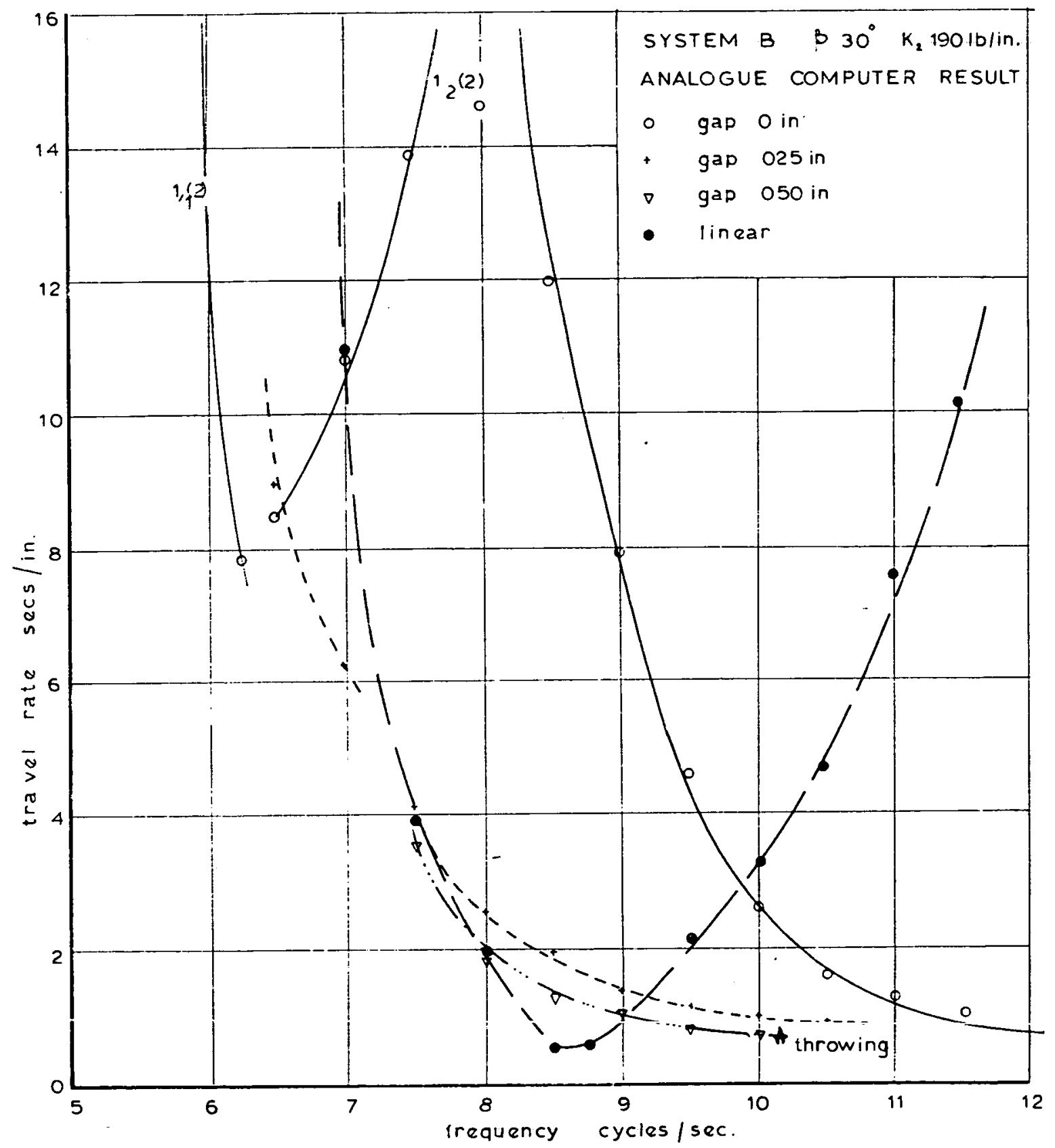

GRAPH OF TRAVEL RATE secs/in. AGAINST FREQUENCY CIS

FIG. $6: 26$ 


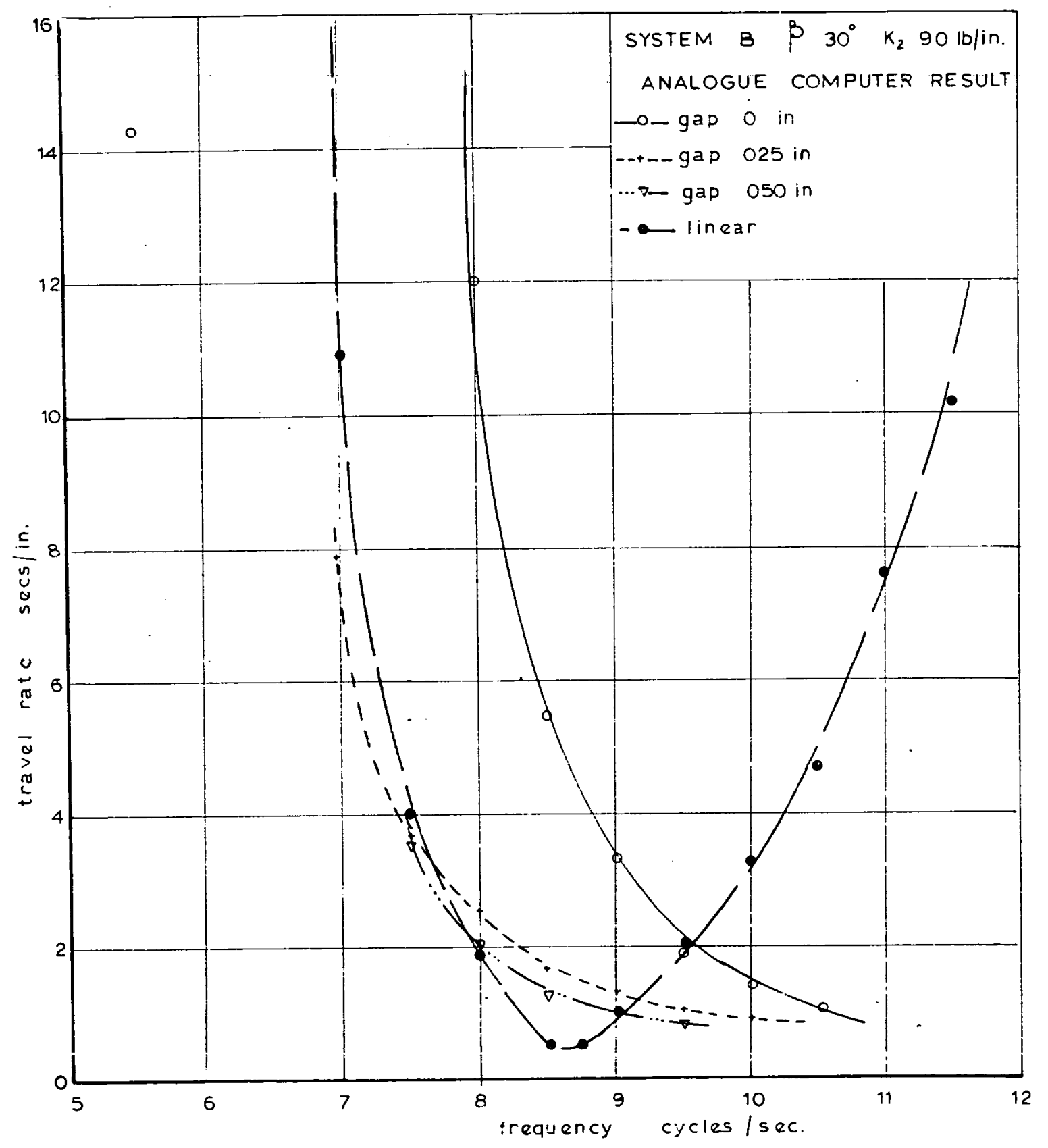

GRAPH OF TRAVEL RATE secs/in. AGAINST FREQUENCY Cls.

FIG. 6:27 
ANALOGUE COMPUTER RESULT

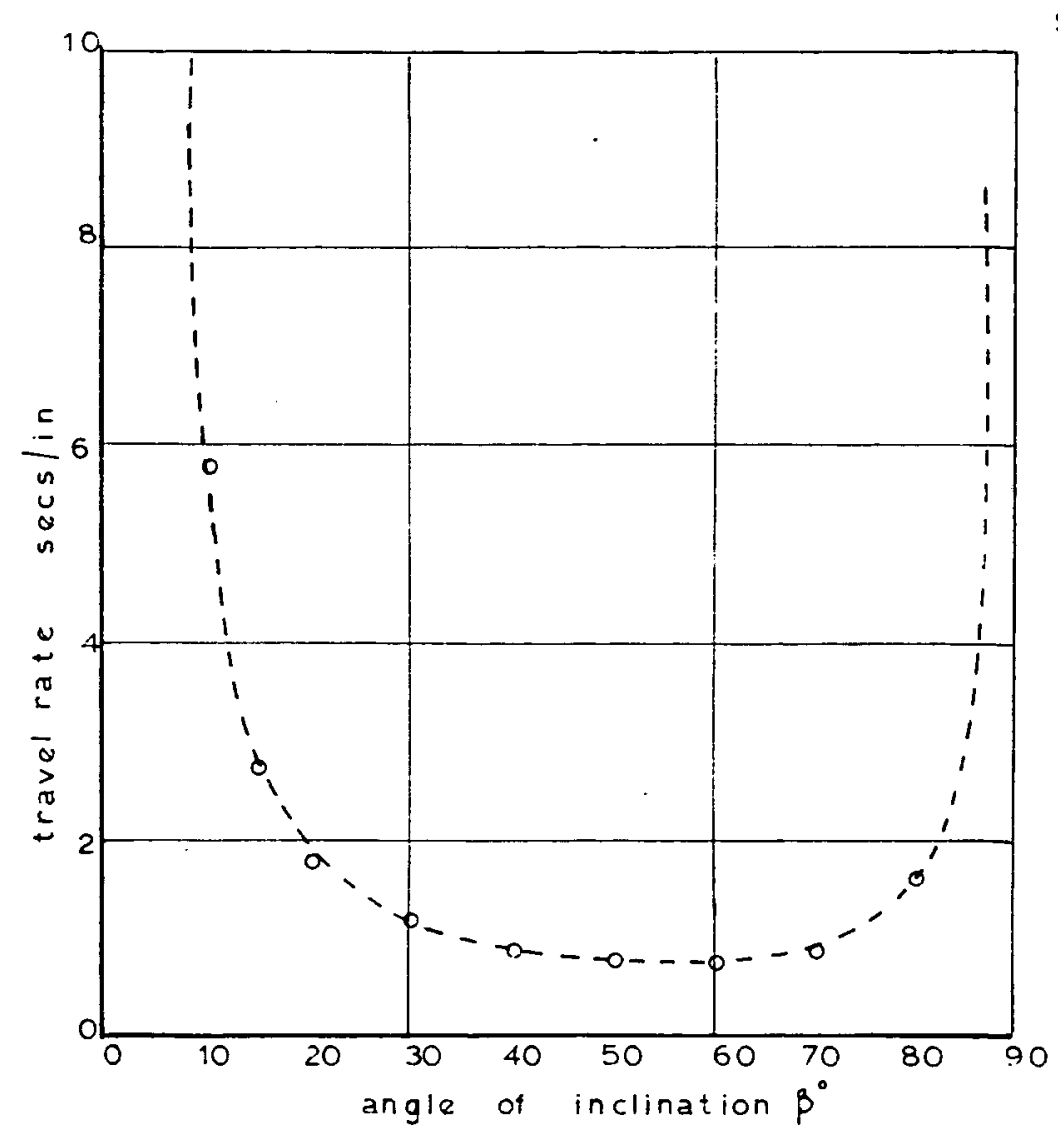

SYSTEM B - LINEAR

FREQUENCY $9 \mathrm{c} / \mathrm{s}$.

FRICTLON $\mu \cdot 12$

MAX ACCLN. $g$

GRAPH OF TRAVEL RATE secs/in. AGAINST ANGLE OF INCLINATION OF CONYEYOR VIBRATION $\beta^{\circ}$

FIG. $6: 28$ 


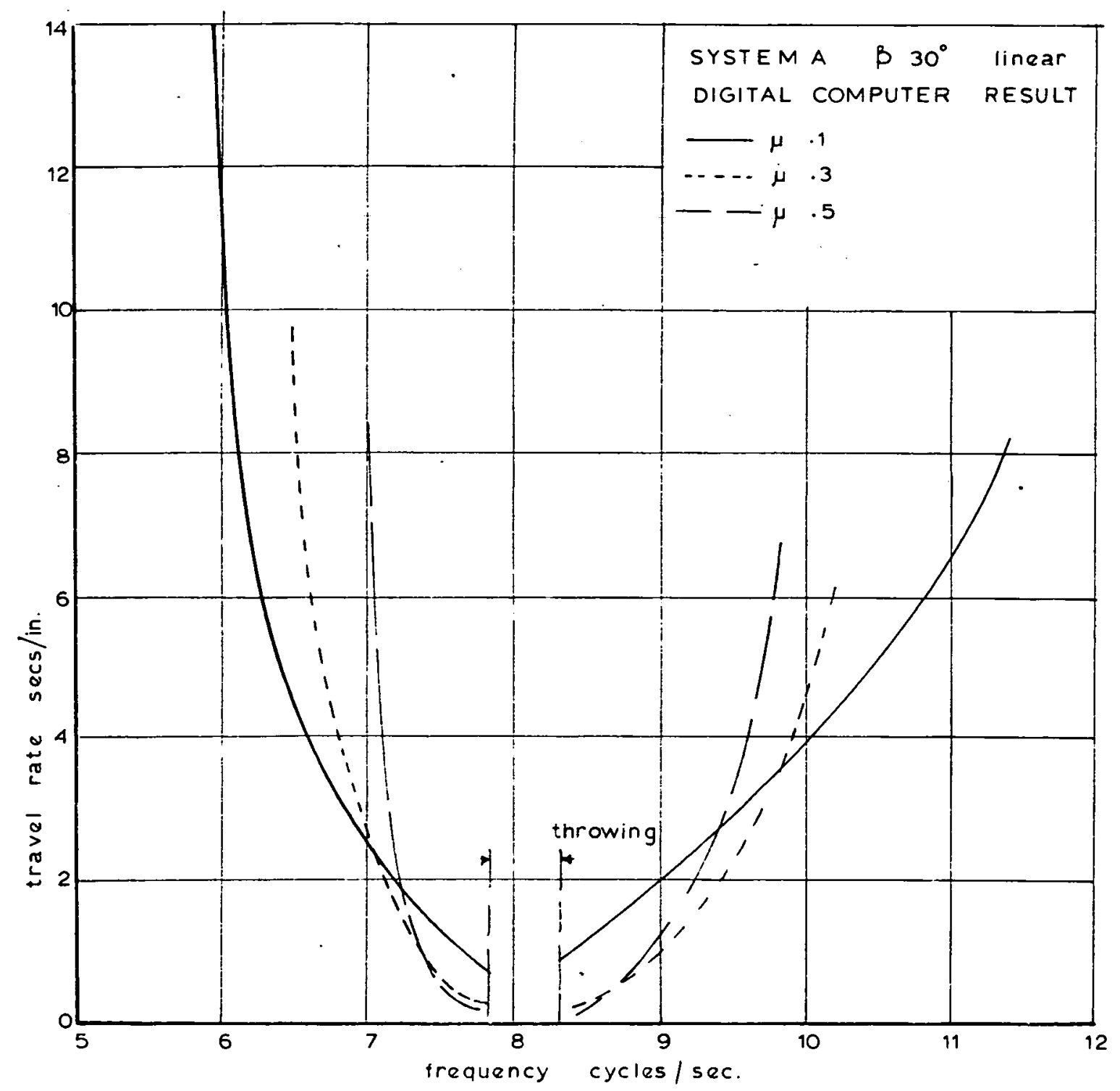

GRAPH OF TRAVEL RATE secs/in AGAINST FREQUENCY cIs. FOR VARIOUS VALUES OF. COEF. OF FRICTION $\mu$.

FIG. $6: 29$ 


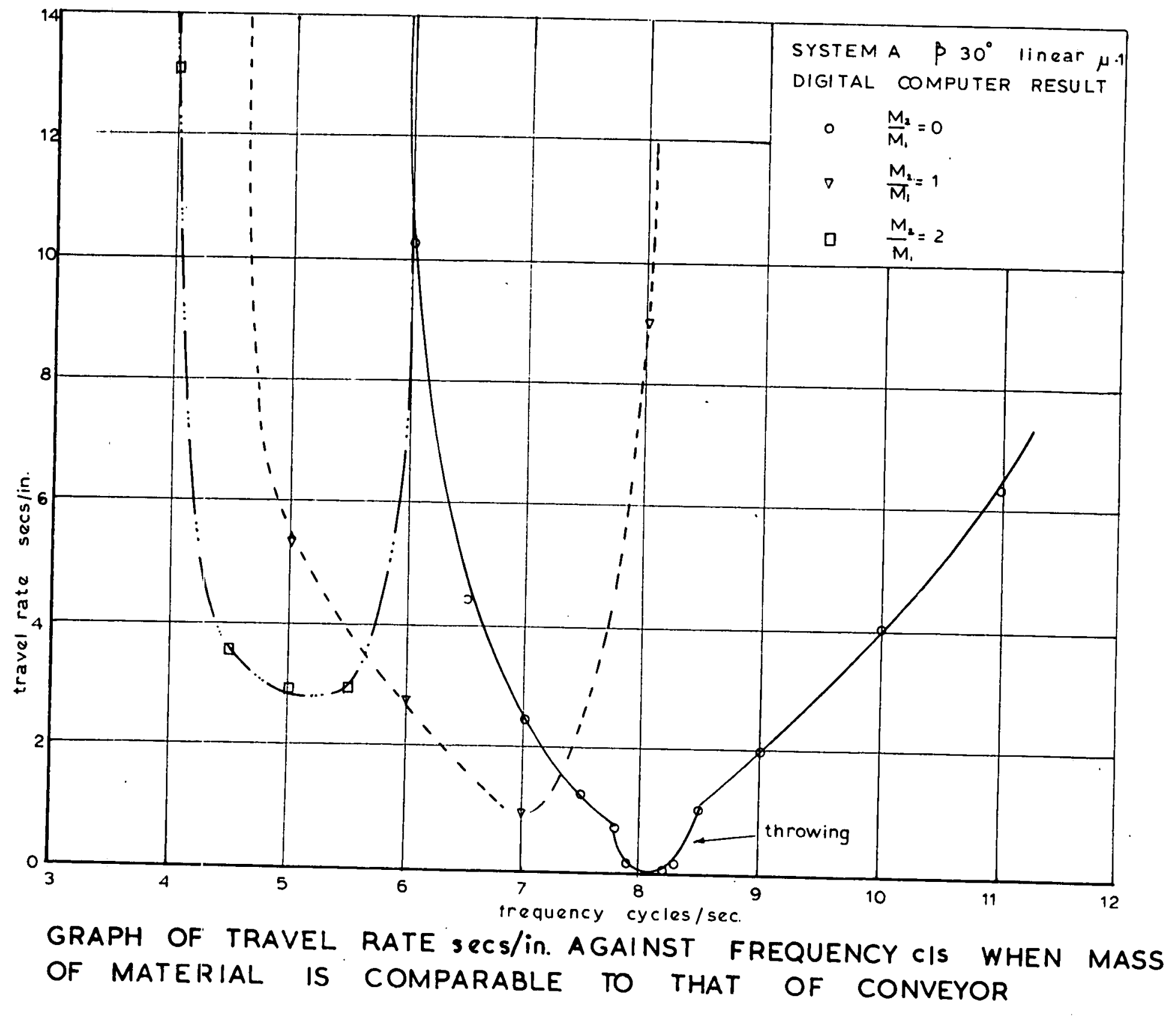

FIG. $6: 30$ 


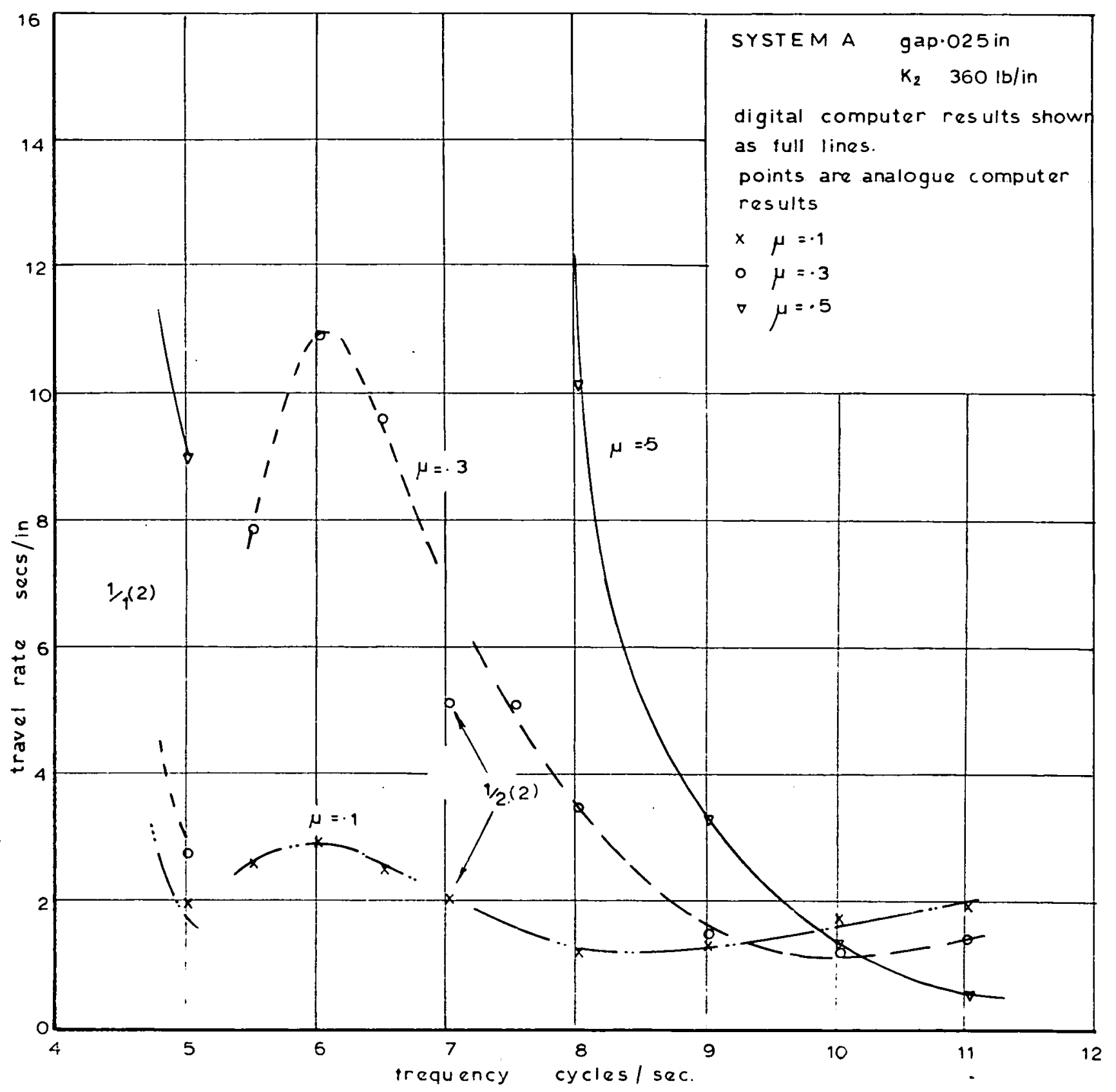

GRAPH OFF TRAAVEL RATE secsjin. AGAiNST FREQUENTCY cis FOR VARIOUS VALUES OF COEF. OF FRICTION $\mu$.

FIG. $6: 31$ 


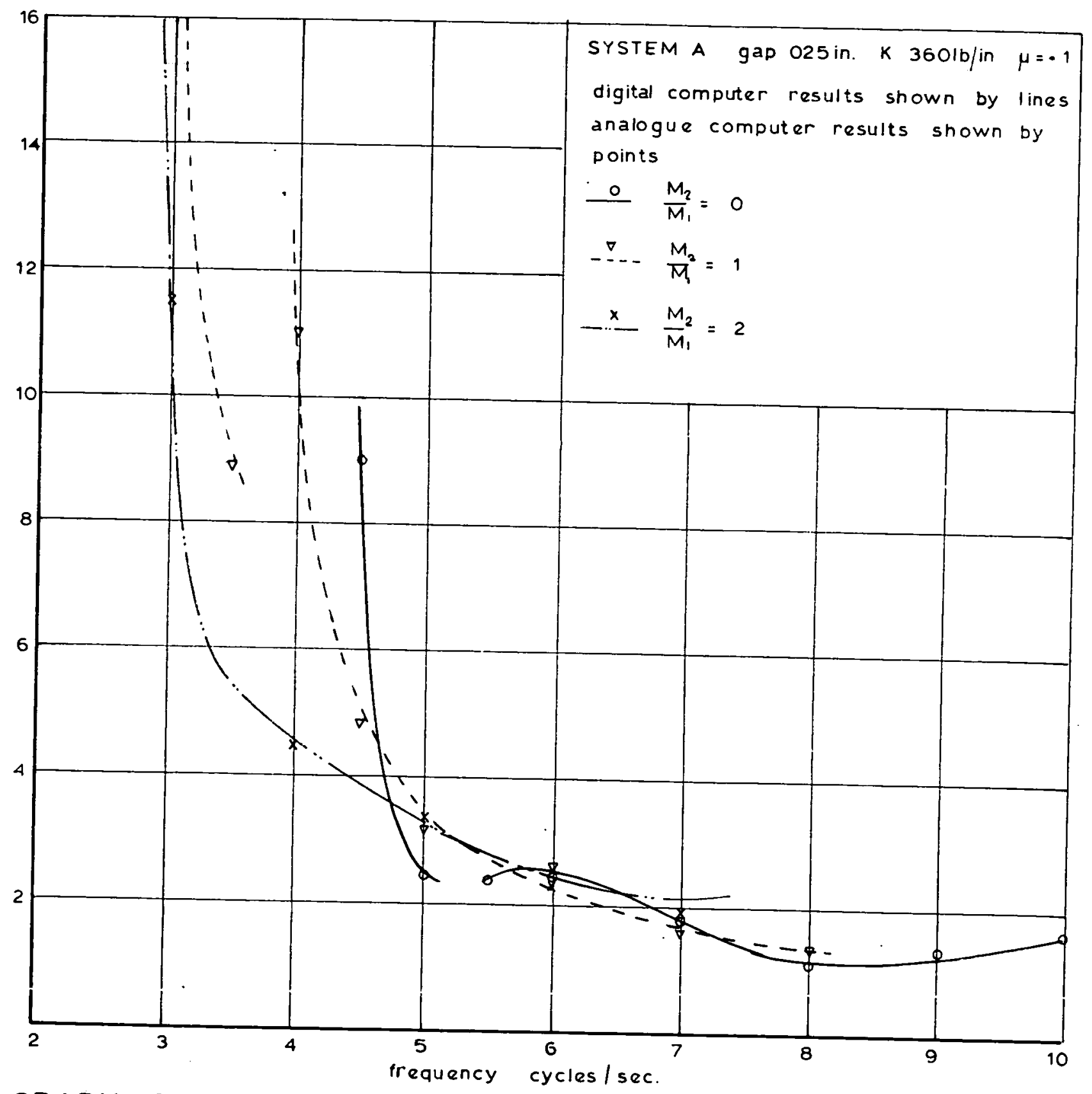

GRAPH OF TRAVEL RATE secs/in. AGAINST FREQUENCY CIs WHEN MATERIAL MASS IS COMPARABLE TO CONVEYOR MASS.

FIG. $6: 32$ 
SYSTEM A DIGITAL COMPUTER RESULT.
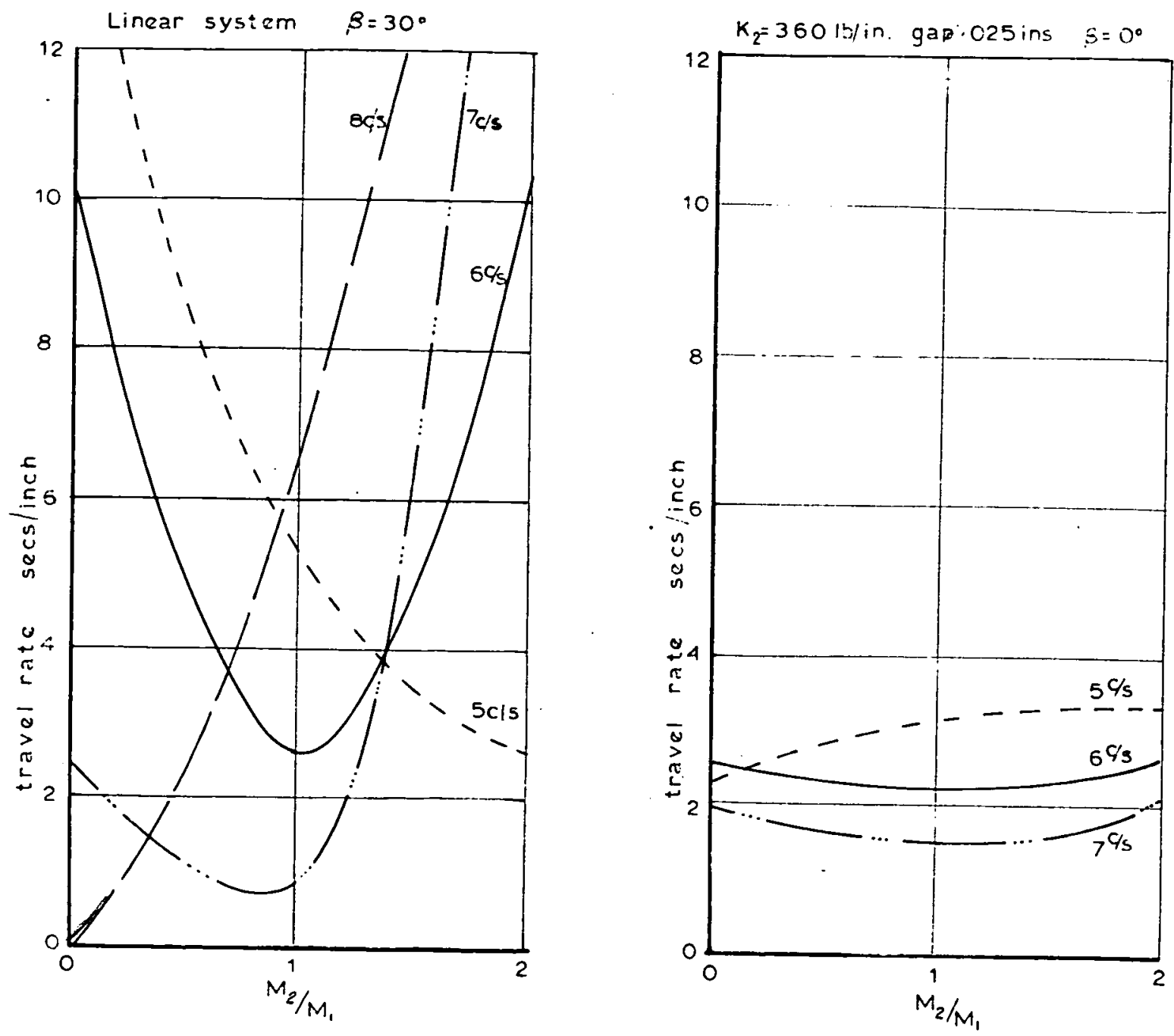

GRAPHS SHOWING DEPENDENCE OF TRAVEL RATE(secs/in) ON MATERIAL LOAD FOR VARIOUS VALUES OF EXCITING FREQUENCY C!S.

FIG. $6: 33$ 


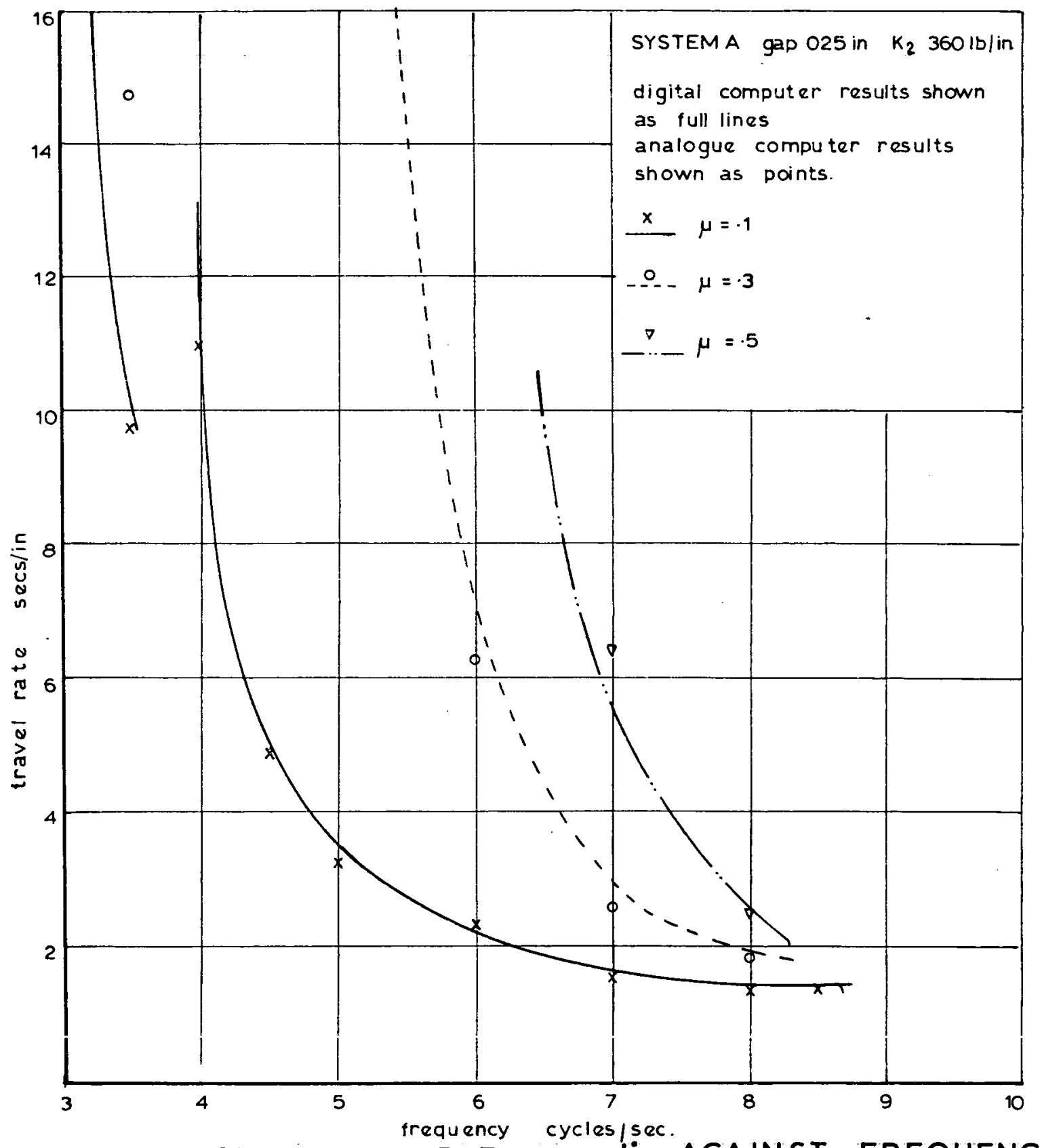

GRAPH OF TRAVEL RATE secs/in. AGAINST FREQUENCY cIs FOR VARIOUS VALUES OF COEF. OF FRICTION $\mu$ WITH MASS OF MATERIAL EQUAL TO THAT OF CONVEYOR $\left(M_{2}=M_{1}\right)$

FIG. 6:34 


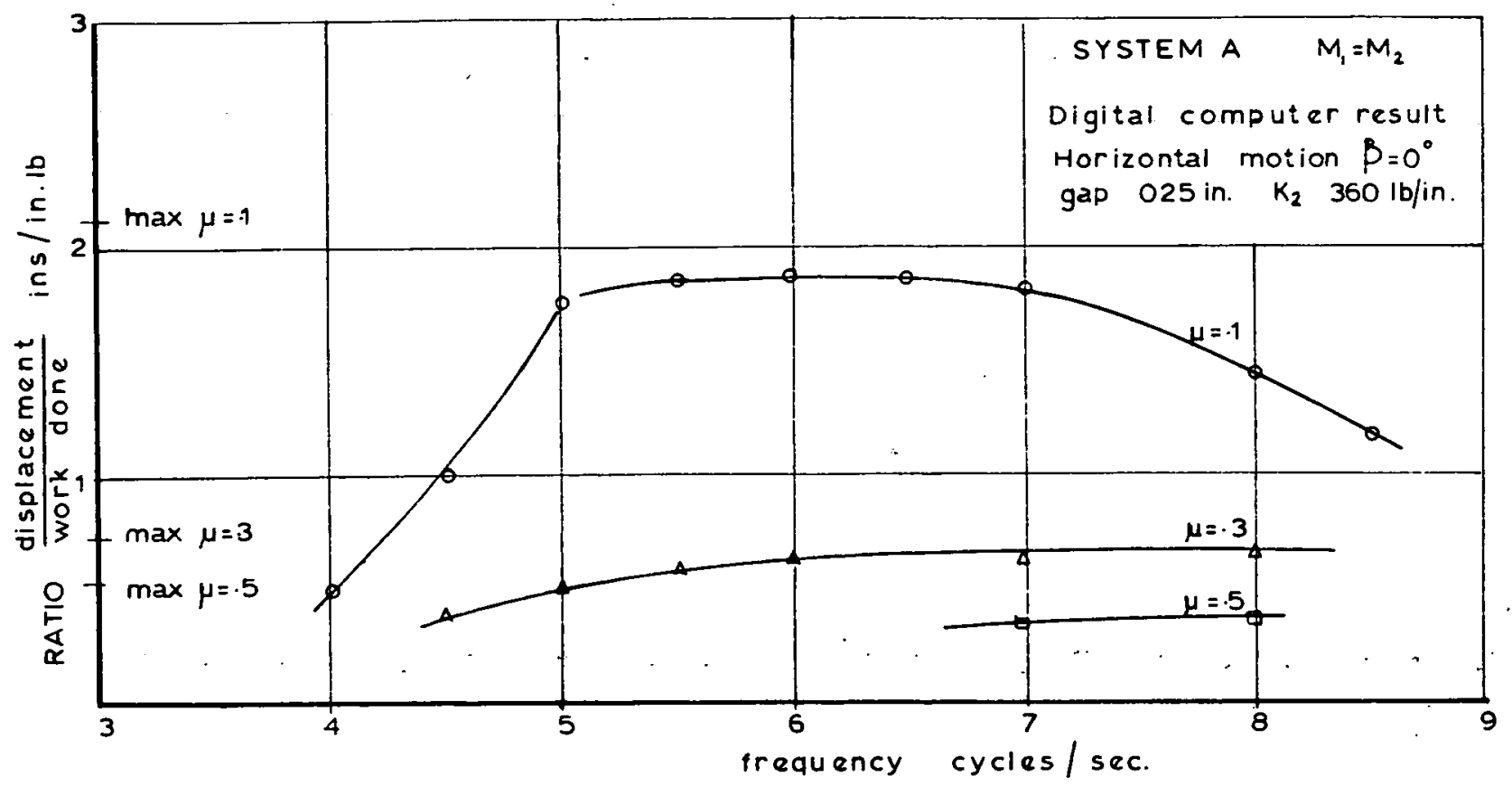

GRAPH OF 'EFFICIENCY' ins /in.lb AGAINST FREQUENCY c/s FOR VARIOUS VALUES OF COEF. OF FRICTION $\mu$.

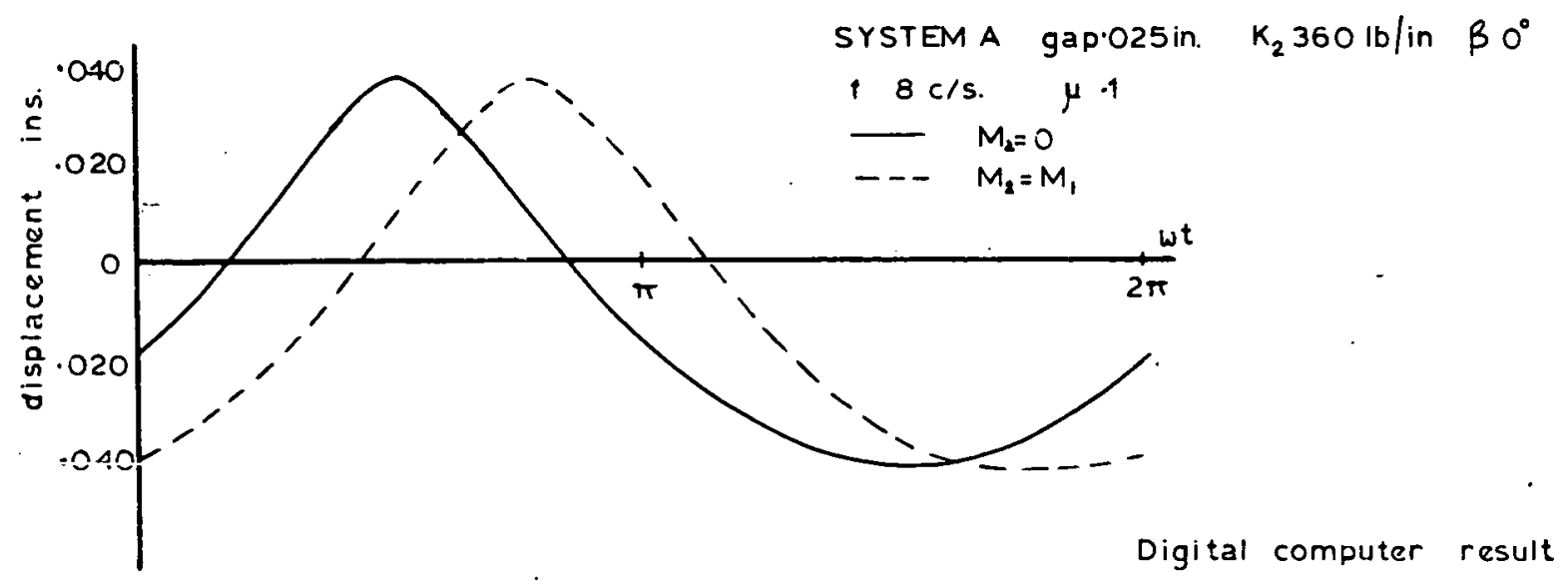

CONVEYOR DISPLACEMENT WAVEFORMS

FIG. 6:35 


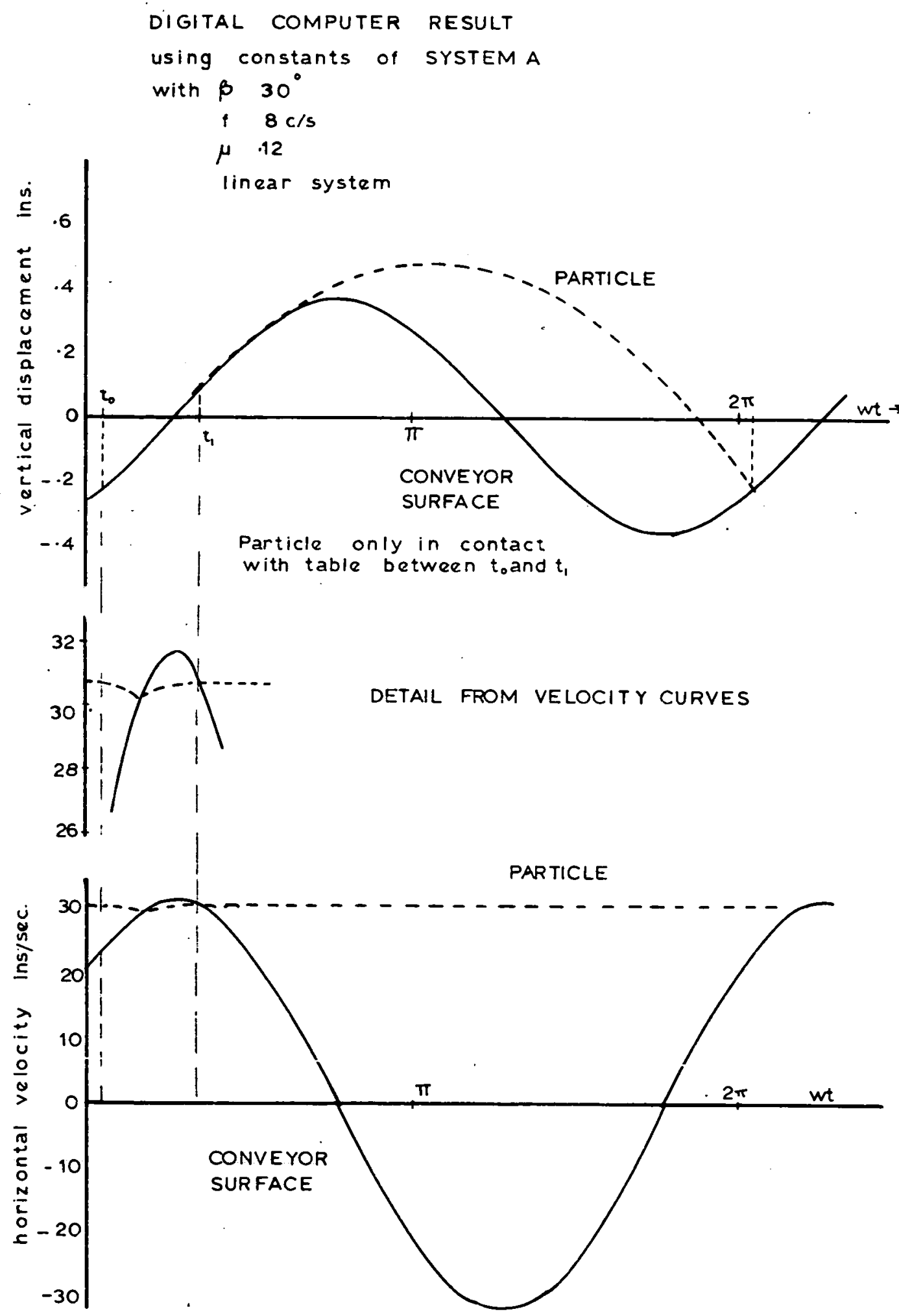

DISPLACEMENT \& VELOCITY WAVEFORMS WITH MAXIMUM VERTICAL ACCELERATION > GRAVITY

FIG. $6: 36$ 


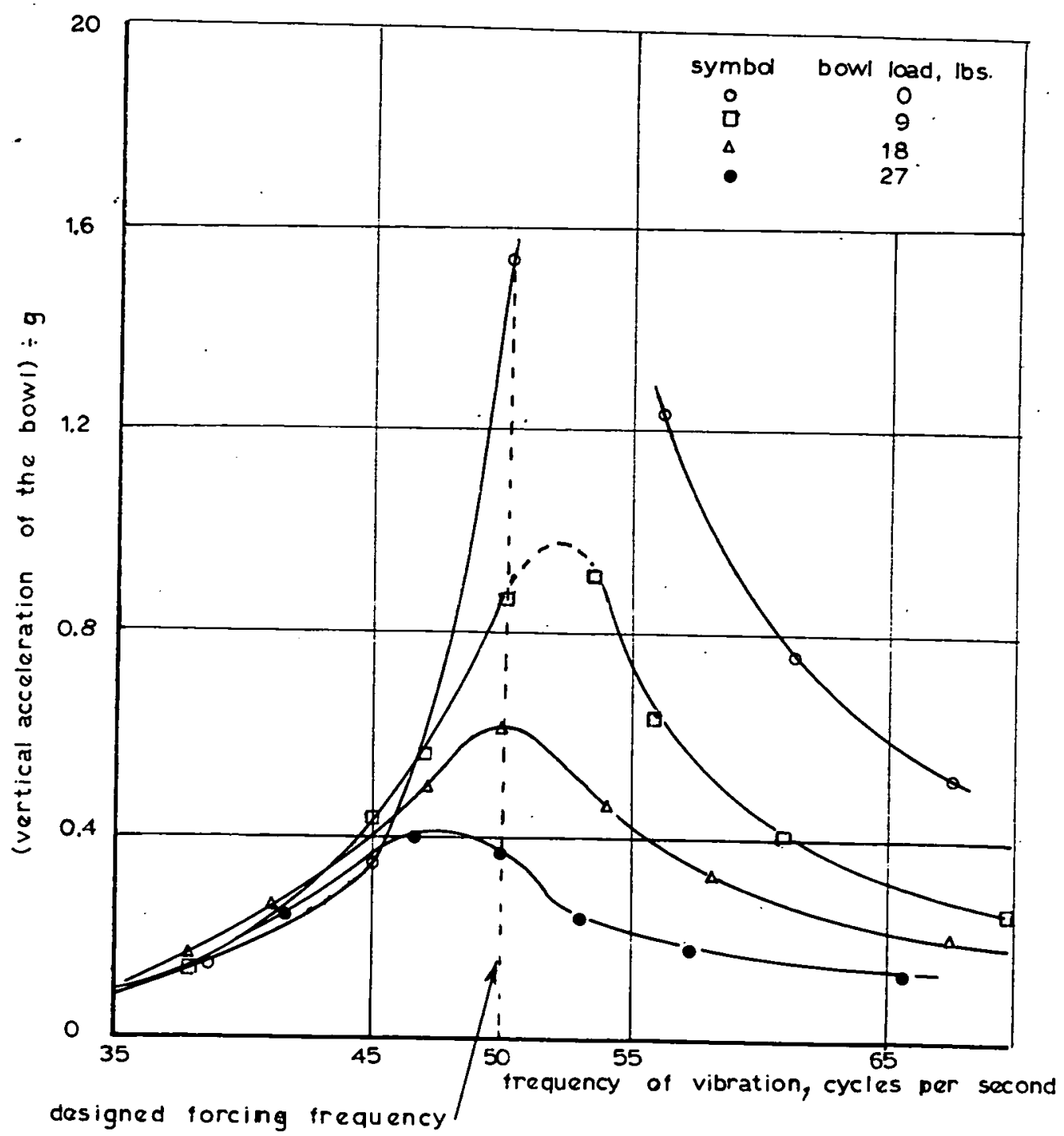

FREOUENCY RESPONSE CUMVES FOR VIRPATORY OOWL FEEDER FOR FOUR VALUES OF BOWL LOAD AND A CONSTANT INPUT TO THE BOWL ELECTRO-MAGNET OF 65 VOLT-AMPS.

RESULT FROM A.H. REDFORD.

FIG. $6: 37$ 


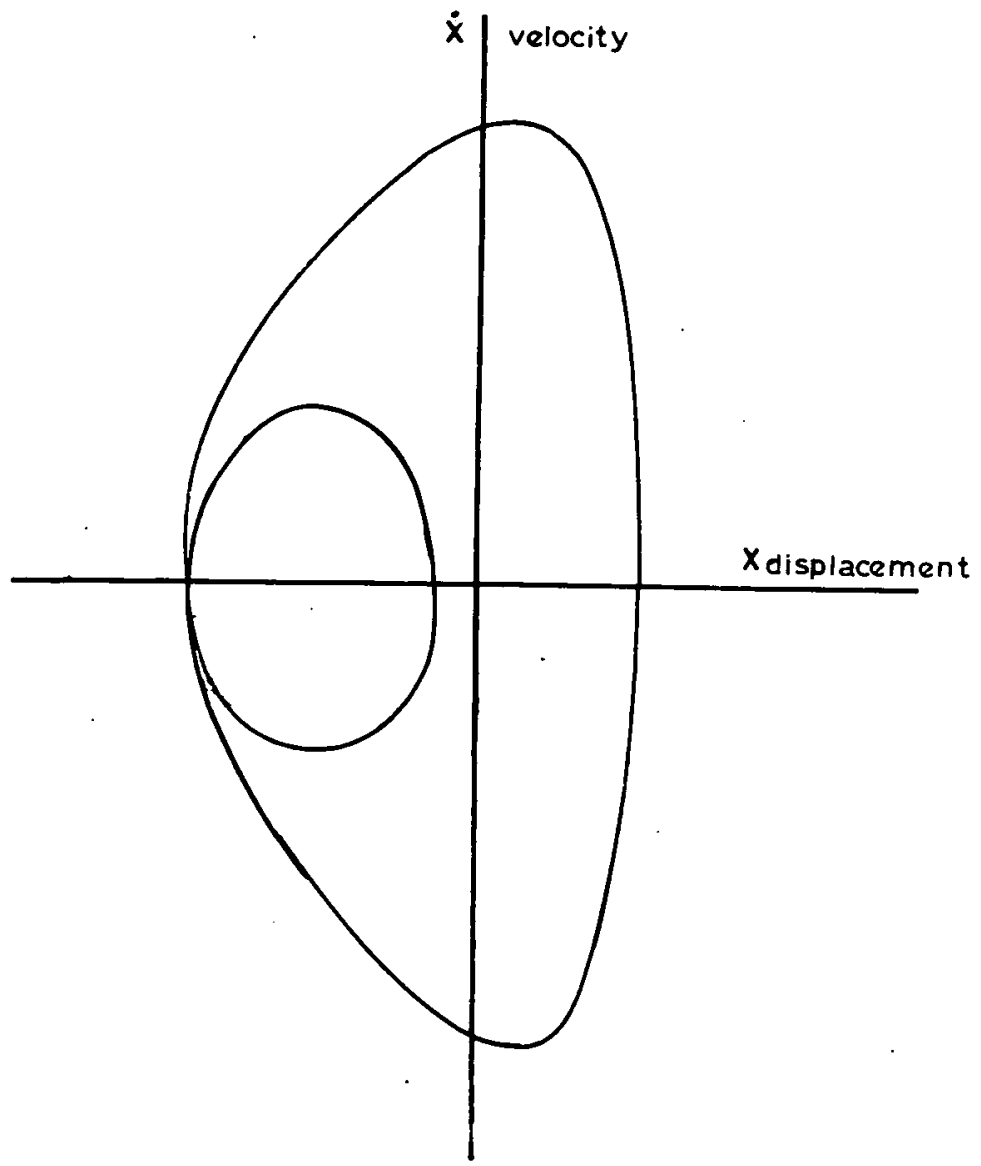

PHASE PLANE TRAJECTORY

HARMONIC SOLUTION $K_{2} 360 / 1 \mathrm{~b}$ in gap 0 ins. $\$ 6.5 \mathrm{c} / \mathrm{s}$.

FIG. $6: 38$ 


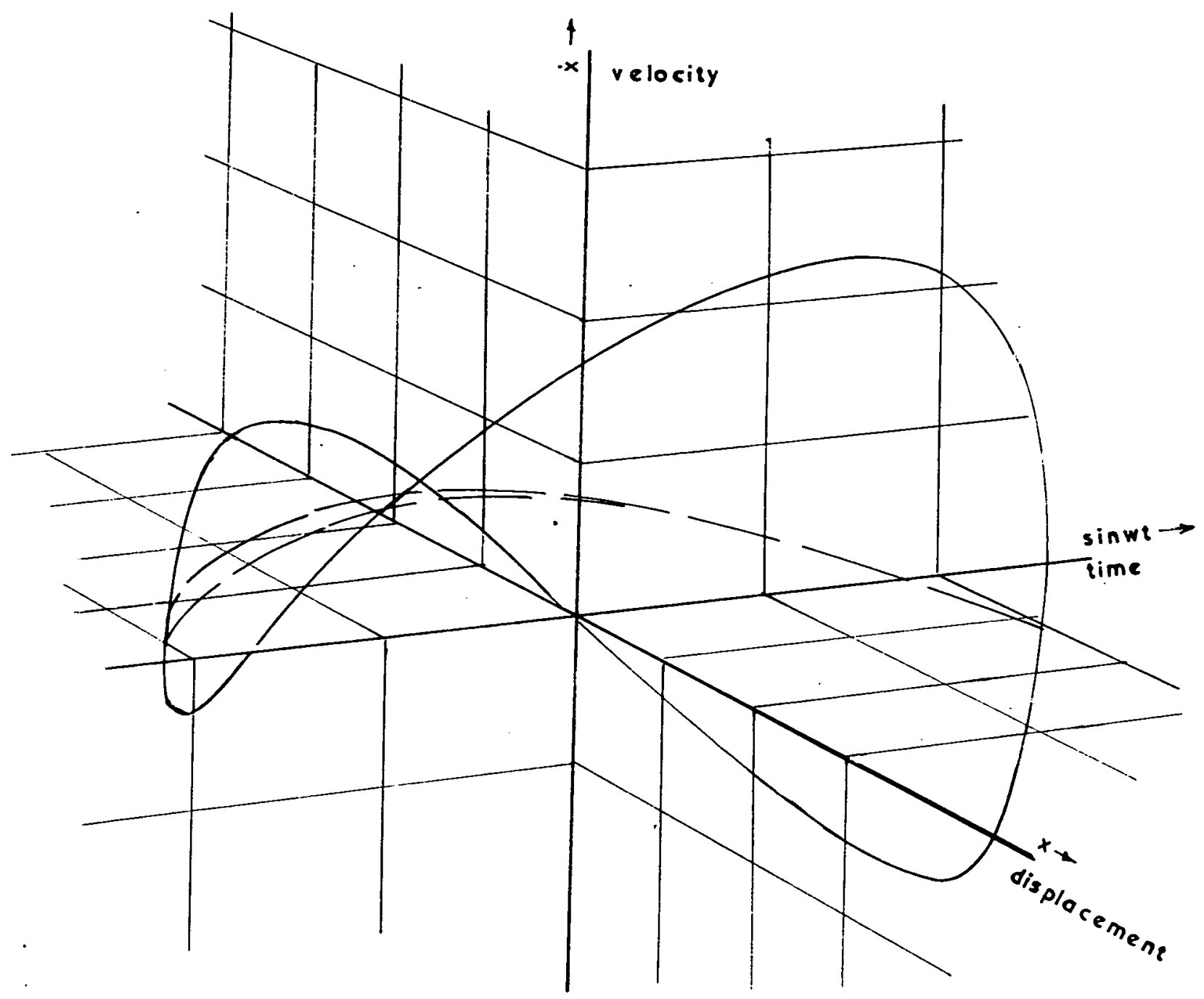

BROKEN LINE PROJECTION IN $X-T$ PLANE

PHASE PLANE TRAJEctory WITH TIME AS 3rd. CO-ORDINATE

FIG. $6: 39$ 

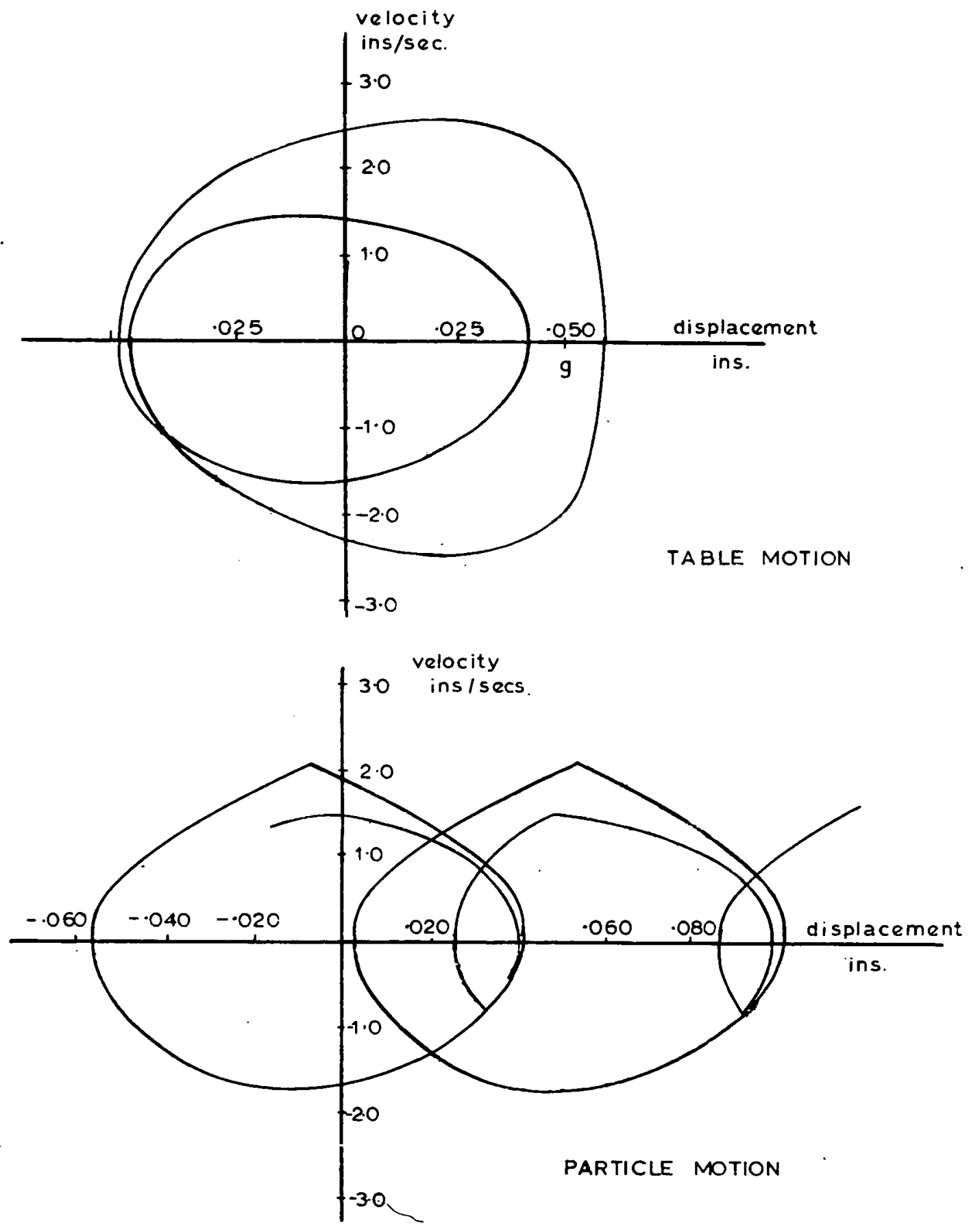

PHASE PLANE REPRESENTATION OF $1 / 2$ SUB - HARMONIC.

DIGITAL COMPUTER RESULT

FIG. $6: 40$ 


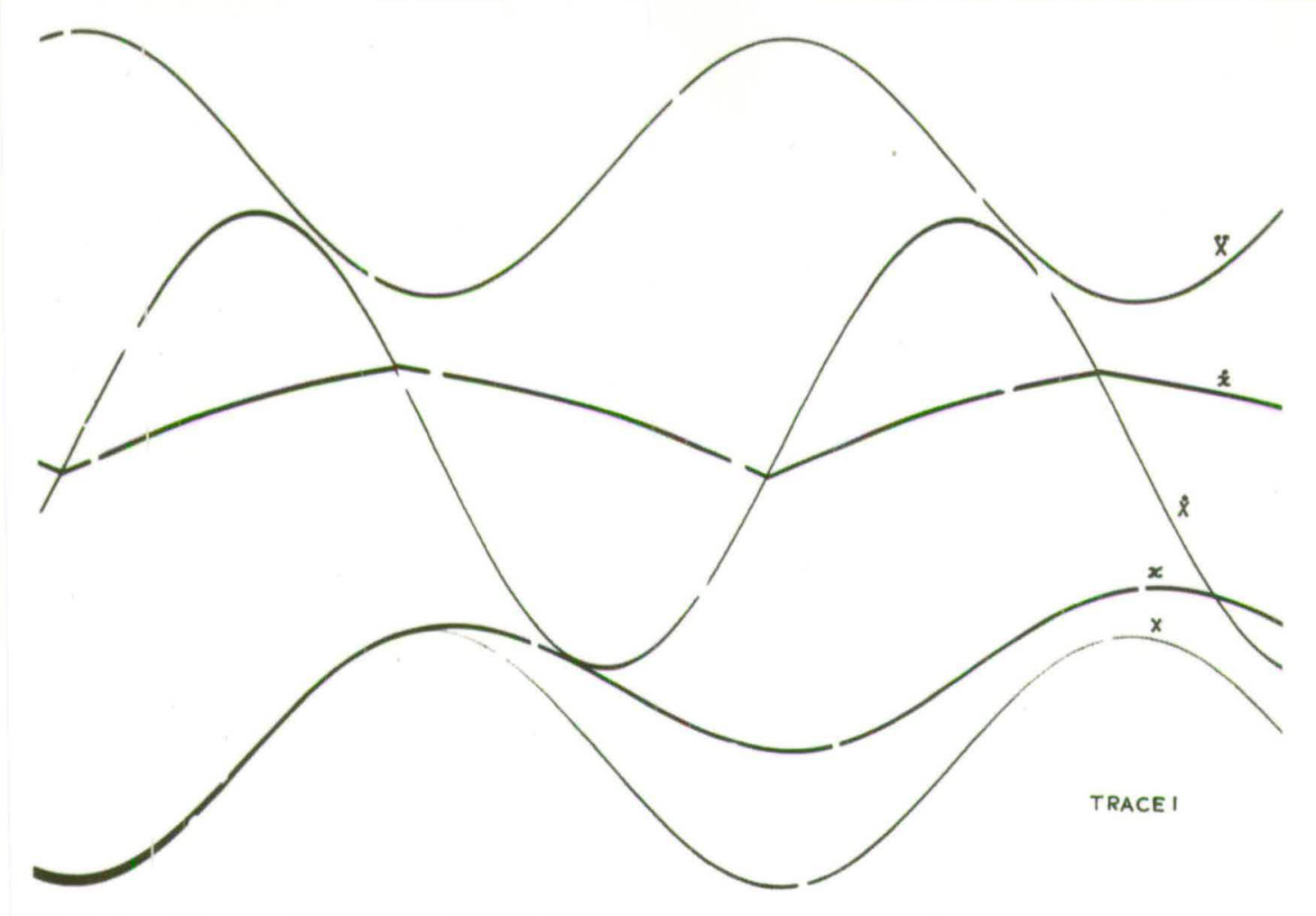

Plate 6.I Linear System

System B; $\beta=30^{\circ} ; \mu=.12 ; f=9 \mathrm{c} / \mathrm{s} ; M_{2}=0$ 

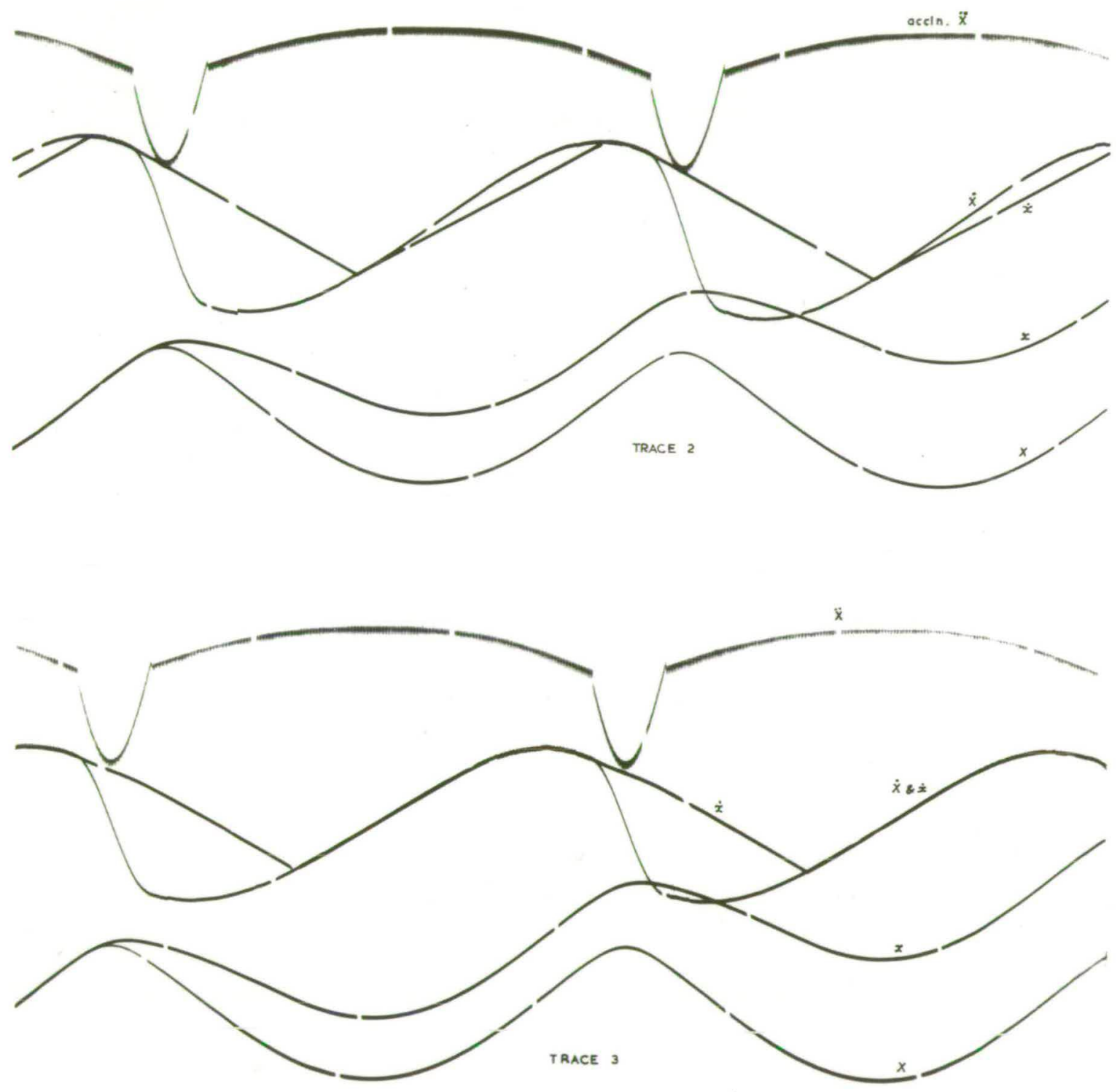

Plate 6.2 System B; gap 025 in.; $K_{2}=360$ Ib./in.; $\mu=.12$;

$\hat{f}=8 \mathrm{c} / \mathrm{s}, \mathrm{M}_{2}=0$

Trace $2 \beta \stackrel{2}{=} 0$

Trace $3 \quad \beta=30^{\circ}$ 

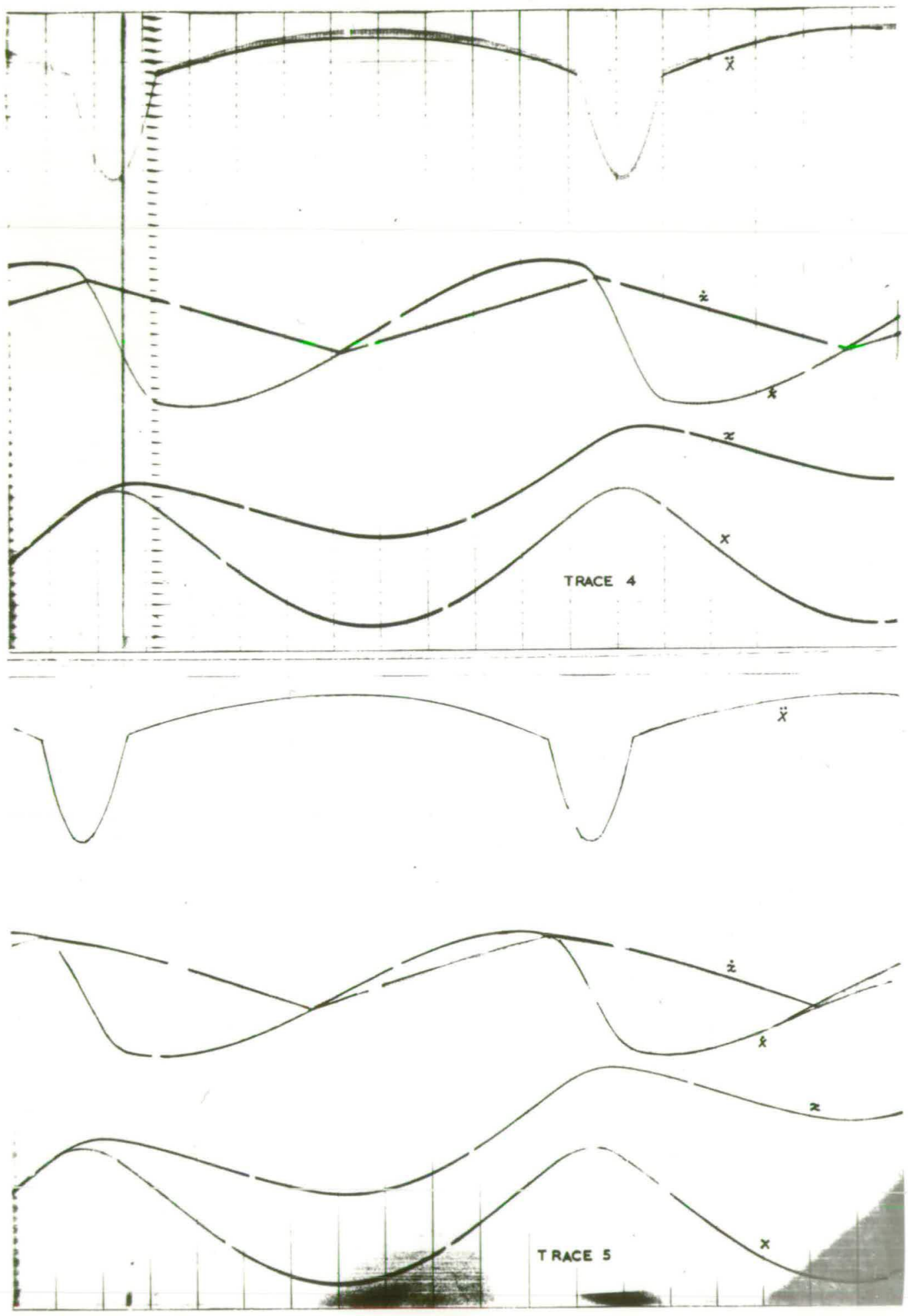

Plate 6.3 System B; gap 025 in.; $K_{2}=360 \mathrm{lb} /$ in.; $\mu=.12$; $f=9 \mathrm{c} / \mathrm{s} ; \mathrm{M}_{2}=0$

Trace $4 \quad \beta=0$

Trace $5 \quad \beta=30^{\circ}$ 

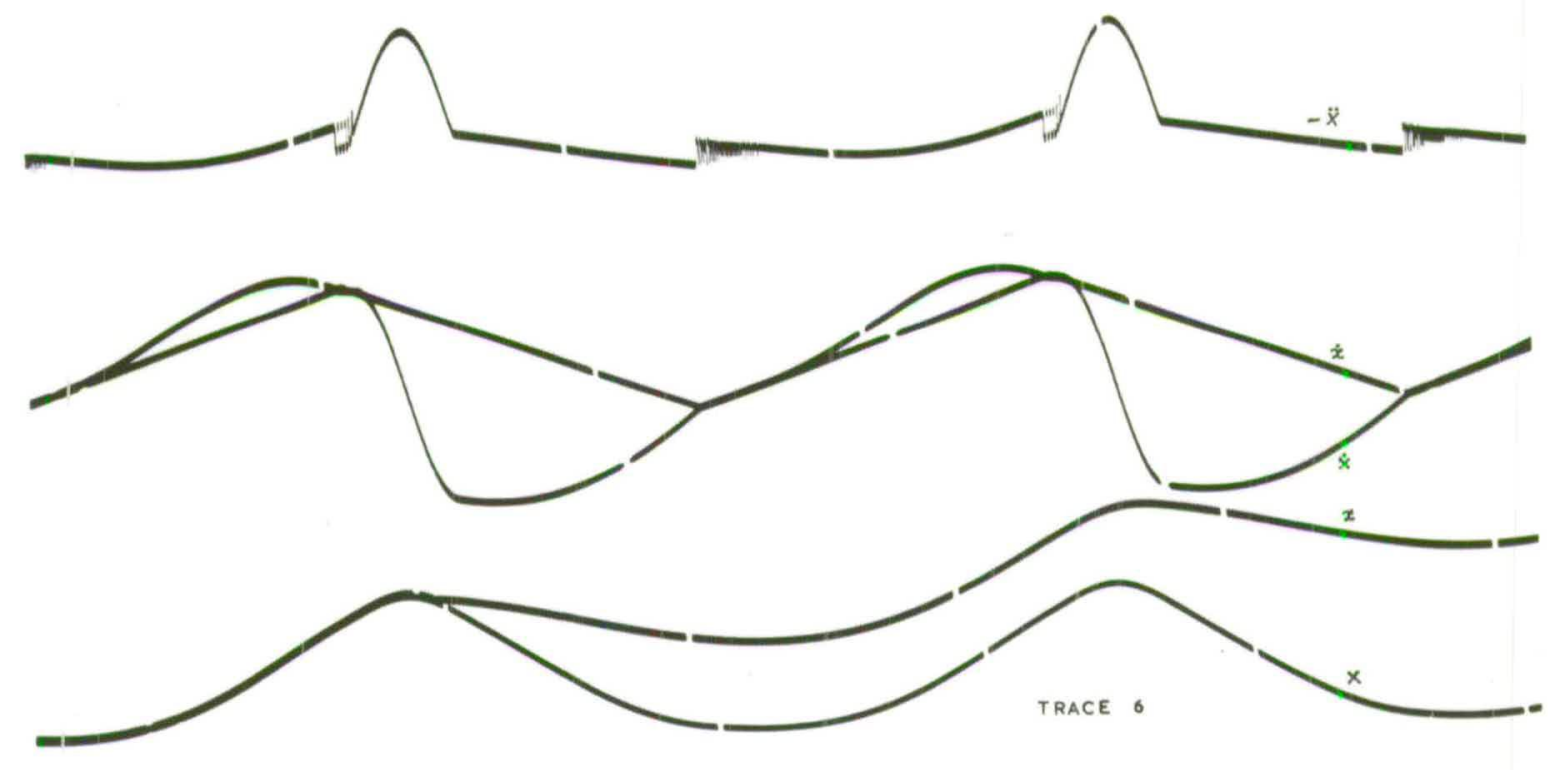

Plate 6.4 System A - Horizontal conveyor motion with a large mass of material being conveyed.

$f=8 \mathrm{c} / \mathrm{s} ; \mu=.1 ; \mathbb{M}_{2}=\mathbb{M}_{1} ;$ gap 025 in.; $K_{2}=360$ lb./in.;

'stick-slip' motion. 


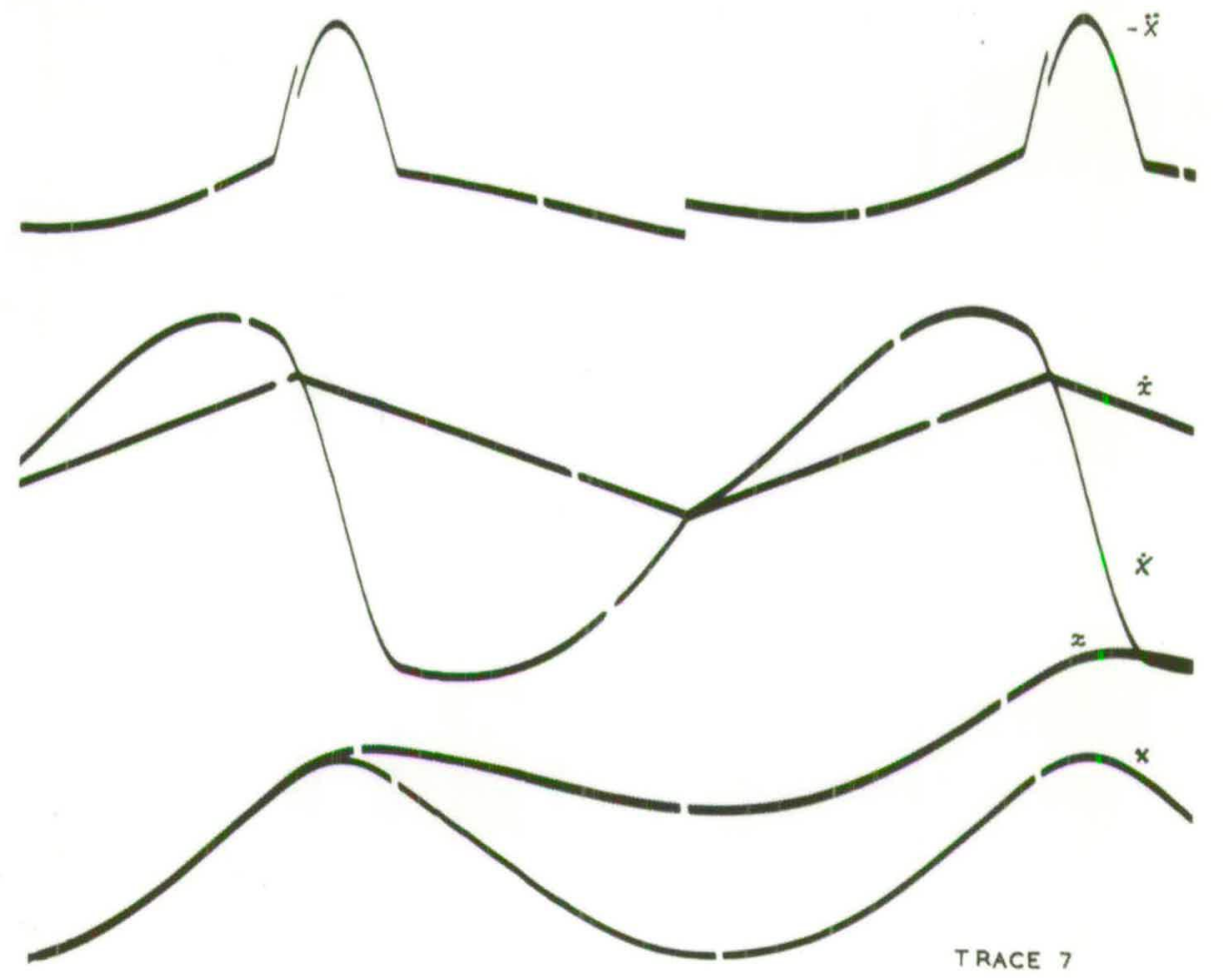

Plate 6.5 System A - Horizontal conveyor motion with a large mass of material being transported.

$f=9 \mathrm{c} / \mathrm{s} ; \mu=\cdot 1 ; M_{2}=M_{1} ;$ gap 025 in $; \mathbb{K}_{2}=300$ lb./in.

'continuous slip' motion. 


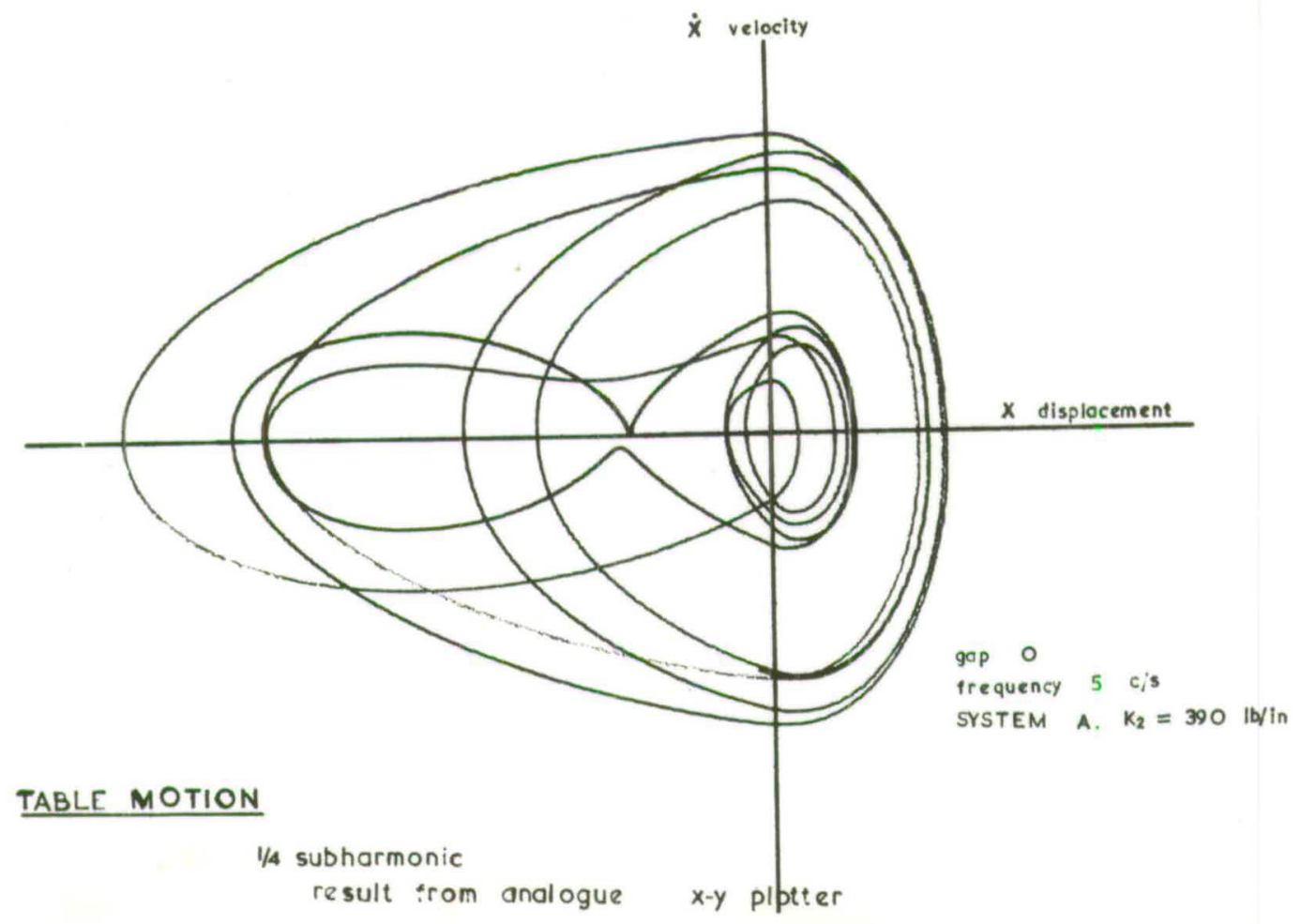

Plate 6.6 'Phase Plane' trajectory of the most complex motion founc. In this case the table makes contact with the hard spring 9 times in 4 cycles of force. This motion was also found on the experimertal equipment. 


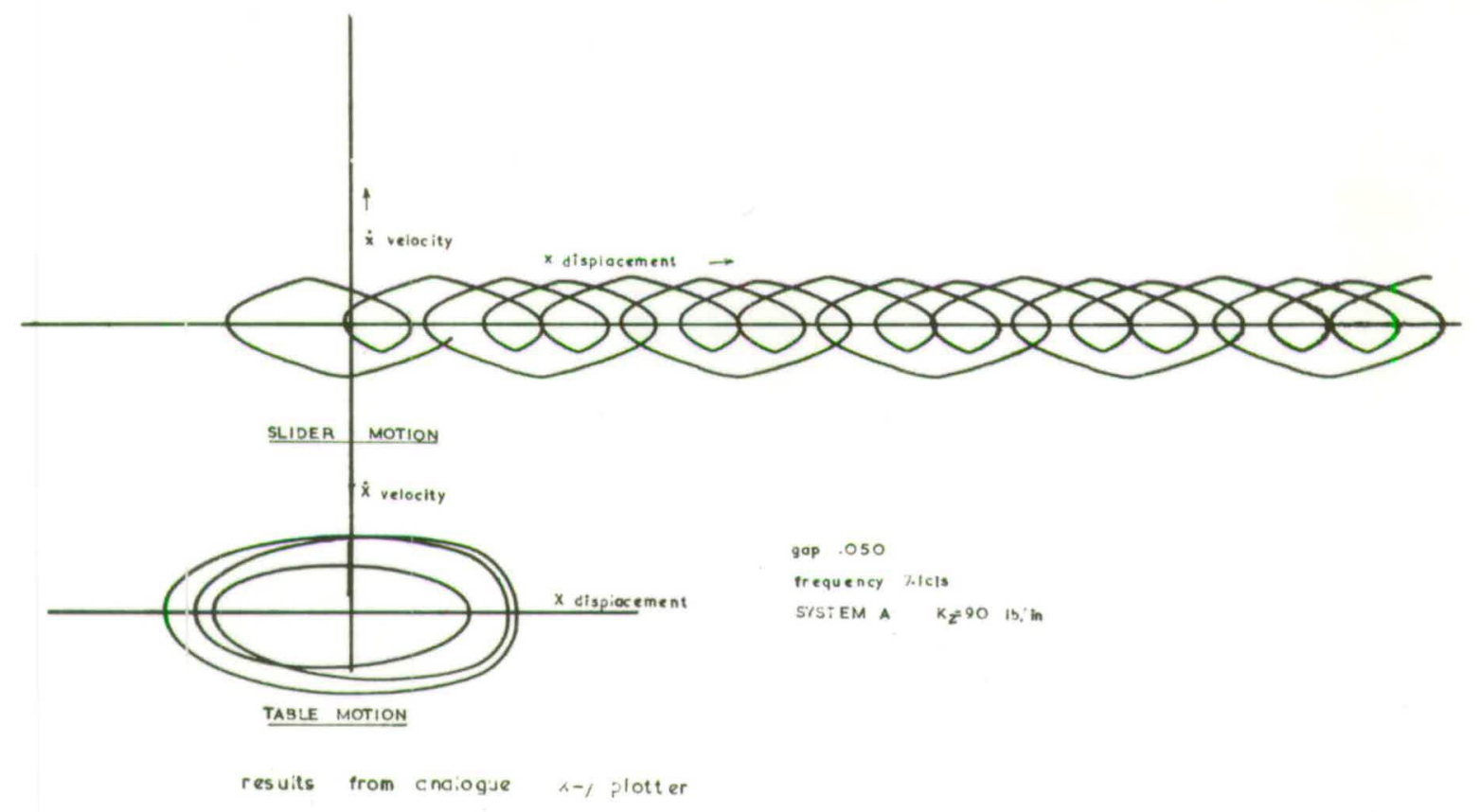

Plate 6.7 'Phase Plane' trajectory of both table and material 


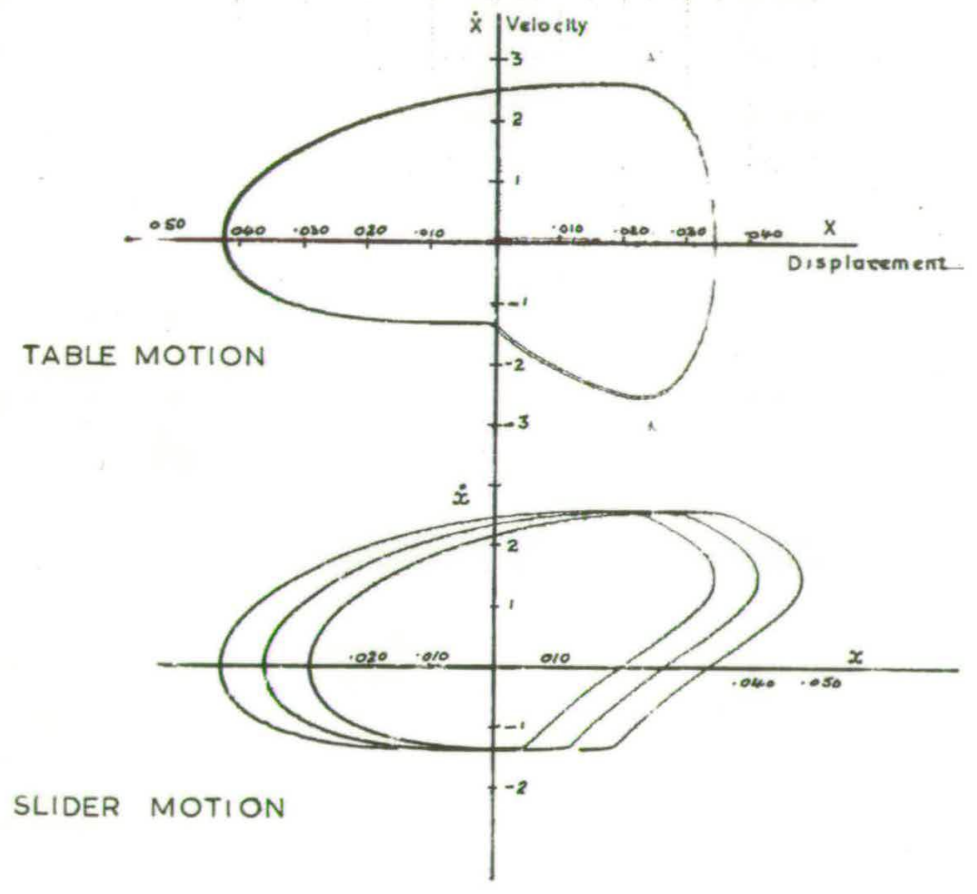

Plate 6.8 'Phase Plane' trajectory for System A with $\mathrm{M}_{2}=\mathrm{M}_{7}$, $\mu=\cdot 3$, gap 025, $\mathrm{K}_{2}=360 \mathrm{lb} \cdot / \mathrm{in} ., \mu=.3,{ }^{2} \beta=0$. The sharp change in the phase plane of the table motion indicates the discontinuity in acceleration when relative slipping ends. 


\section{CHAPTER 7}

7. CONCLUSIONS

\subsection{Objectives of the Investigation}

This research was undertaken to study the performance of a resonant vibratory conveyor in which the spring force was unsymmetrically nonlinear, and to evaluate this type of conveyor compared with the usual resonant linear systen. This involved two branches - the frequency response of the system and the effect of velocity waveform on travel rate. Previous research has established the mechanism of sliding by which rigid bodies are transported. Some further confirmation was required of the movement of bulk materials. Since non-linear systems do not allow results to be presented in non-dimensional form which could be applied to other systems, analogue computer circuits and digital computer progranmes were required which would allow calculation of the transport performance of any conveyor. Those computer methods were to be used to study the performance of conveyors when the material being transported was heavy and the usual idealisation of a weightless particle could not be used.

\subsection{Over-all results and conclusions}

The agreement obtained between the computer results and those from experimental work confirm the work of previous investigators in that the inechanism of material sliding is confirmed. If the conveyor surface displacement is defined and the coefficient of friction between the material and the conveyor surface given, it is possible to predict accurately the transport velocity of material. It is obvious that the assumption that the static and kinetic coefficients of friction are identical has produced no error. Simple experiments show that a bulk 
material behaves as a single rigid body. Nonlinearities such as the bilinear spring force in this thesis must be defined from some reference position. In this thesis the gap was defined from the equilibrium position of the table with no added material load. When the inclination is at an angle and there is a heavy load on the conveyor the gap setting is increased, and this destroys the tuning of the system. There is of course no reason why the spring characteristic should not be defined with reference to the equilibrium position of the conveyor under the material load which is expected.

The principal conclusions are as follows

1. If an unsymmetrical hard non-linearity is introduced to the restoring spring of a linear vibratory conveyor, the resulting system will offer comparable transport rates to those produced by the linear system. To obtain the best performance the new system will have to be excited at a higher frequency than the linear system.

2. The travel rate of material on the non-linear conveyor will, under optimum conditions be much less dependent on accurate tuning than is the case for a linear system.

3. The design frequency of operation of a non-linear conveyor must take account not only of the 'jump' frequency which will impose an upper limit, but also of a lower limit defined by regions of ultra-harmonic and ultra-subharmonic response which may occur.

4. The performance of a linear vibratory conveyor is adversely affected if the material is heavy. It is not possible to maintain the tuning of such a sustem to include wide ranges of material load.

5. While a linear conveyor depends on an inclination in the vibration, usually between $30^{\circ}$ and $60^{\circ}$, a non-linear vibratory does not in general benefit from inclined vibration. 
6. The performance of a horizontally vibrating non-linear conveyor is almost independent of material loading over wide ranges.

7. Theoretical analysis gives expressions for absolute maximum performance of a vibratory conveyor in terms of the coefficient of friction between the load and the conveying surface and the maximum acceleration of the conveyor. These results show that the maximum transport velocity is inversely proportional to the frequency of excitation. The displacements which give these maximum travel rates are extremely non-sinusoidal. For given maximum accelerations three cases of motion were considered. Best transport velocities occur when the horizontal and vertical displacements are independently applied, and the case when they are components of a single inclined displacement gives better transport than does purely horizontal displacement.

8. Both the analogue computer circuit and the digital computer programme give accurate predictions of travel rate. The former was only developed to include cases where the maximum vertical acceleration is less than gravity. Particle transport could be calculated when the vibration was at an angle of inclination, and heavy loads were included when the conveyor motion was horizontal. The digital programme gives results for particle and heavy loads with both horizontal and inclined vibration and was expanded to include throwing motion with the material idealised as a particle. Whose waight is negligible compared with 7.3 How these results affect the design of a conveyor.

the table weight.

It is regretted that discussions of ultra-harmonic and ultrasubharmonic response in Appendix E were so inconclusive. In the absence of any convenient theoretical analysis recourse must be made to analogue computing methods. Because of the greater insight which can be obtained from analogue methods these should be used even when it is 
intended to use digital methods to check the final design.

The nature of the non-linear spring force used in this study is by no means the only one possible though it may well be found to be a convenient one. Large peak accelerations are produced and these might prove difficult to isolate from surrounding plant and require a more massive foundation. Balanced impact devices could help to reduce these forces on the mounting. The degree of non-linearity to be used would depend on the variation in operating conditions expected. Where large fluctuations in load occur the non-linearity should be larger. The effect of various gap settings of the hard spring used in this investigation was not fully investigated, but it is expected that the static deflection of the linear system under a force equal to the exciting force to be used would give a useful range of frequency at which the conveyor could be operated. Damping is not such an easy problem as in the linear case. While light damping would allow the upper frequency limit to be increased, it may also contribute to the presence of regions of ultra-harmonic and ultra-subharmonic motion. While these regions may give good travel rates if the system was exactly tuned to operate in a particular region, sudden changes in travel rate may occur if the response changes from one region to another. When a heavy load is transported the damping introduced by the coulomb friction of the material load will suppress the presence of these regions. One of the drawbacks of a nonlinear system is that if the jump frequency is exceeded the conveyor will probably stop transporting.

The significance of the result of Appendix $B$ should not be neglected. Recent interest in exciting a linear system sinusoidally, the horizontal and vertical components being separately applied with a phase difference between them has been shown to be merely a special case 
of a more general expression. If a highly sophisticated conveyor was required research on the possibilities of combining two non-linear systems would be useful. Theoretically at least this has a greater potential than using sinusoidal displacements.

\subsection{Areas for further studies}

The immediate need is for further analytical study of systens which are markedly non-linear. Until now most of the mathematics on non-linear systems have been concentrated on quasi-linear cases. While computer techniques give good results they cannot yield the same insight into the behaviour of a system as an analytic approach, though it is appreciated that repeated results would require to be obtained from computers. Further experiments are required to attempt to present some approximate rule by which system parameters could be chosen to give a desired travel rate. The improved efficiency which is predicted if the conveyor has its horizontal and vertical components excited separately, not only with a phase angle but with non-linear displacements as shown in Appendix B would make the construction of such a conveyor most interesting. The experiments on sard were very tentative and a more complete investigation of the behaviour of granular media, particularly in throwing motions, would be valuable. The throwing process when a heavy material load is involved is one which would be most interesting to analyse. Finally the stability of material being transported would we worth further study. It is expected that throwing motion is only suitable for granular media, but it has been shown that even under purely horizontal surface motion the travel rate of a component could be seriously affected by rocking motion. In feeding components into automated processes it is important that their alignment be maintained, and how various conveyor motions contribute to this could also be investigated. 
BIBIIOGRAPHY

1. ATKINSON, C.P. and HEFLINGER

2. BERRY, P.E.

3. BERRY, P.E.

4. BOOTH, J.H. and MCCALLION, $\mathrm{H}$.
'Subharmonic and superharmonic oscillation of a bilinear vibrating system?

J. Franklin Inst. 1956, p.185.

'Research on Oscillating Conveyors'. J. agric Engng. Res. 1958, 3(3) 249.

'Basic Theory of Low-acceleration Oscillating Conveyors'.

J. agric Engng. Res. 1959, 4(3) 204.

IOn Predicting the Mean Conveying Velocity of a Vibrating Conveyor'. Proc. Inst. Mech. Engng. Vol.178; Pt.I, No.19, 1963/64.

4a. also BOOTH, J.H. 'Mechanics of a Vibrating Conveyor' M.Sc. Thesis, University of Nottingham, 1960.

5. BRICKMAN, A.D.

'The Vibratory Transport of Bulk Materials' Ph.D. Thesis, The University of Michigan, Nov. 1963.

6. BROCK, J.E.

'An iterative numerical method for nonlinear vibrations'. Jour. App. Meoh. Trans. A.S.M.E. Vol. 74,

7. de COCK, H.G.

'Vibratory Feeders'. Phillips Tech. Review, 24, 3, 1962/63.

8. EFSTATHIADES, G.J. 'Combination Tones in Non-Linear Systems'. Muirheads 'Technique' Oct. 1966.

9. GRAHAM, D. and McRUER, D.

10. GUTMAN, I.

11. den HARTOG, J.P.

'Analysis of Non-Linear Control Systems' John Wiley, New York, 1961.

'Vibratory Conveyors'. Engineers Digest, May, 1963.

'Forced Vibrations with Non-Linear Spring Constants'.

Trans. A.S.M.T. Paper APM-54-15.

12. HAYASHI, C.

13. HUSKEY and KORN
'Nonlinear oscillations in physical systems'. McGraw-Hil1, 1964.

'Computer Handbook'. icGraw-Hill. 
14. INGLIS, C.

15. KENNEDY, C.C. and PANCU, C.D.P.

16. KIRK, C.L.

17. KLUCE, W.

18. LEVENSON, M.

19. LEVENSON, M.

20. LUDEKE, C.A.

21. MAGMARTIN, I.P.

22. MARTIENSSEN, 0 .

23. McLACHLAN, N.W.

24. MINORSKY, N.

25. MITROPOLSKY and BOGOLINBOV

26. $\mathrm{PAZ}, \mathrm{M}$.
'Applied Mechanics for Engineers'. Cambridge University Press.

'Use of vectors in vibration measirement and analysis'.

J. Aero. Sci. 1.947 14 p.603.

'Particle motion on direct drive coal screens'. Colliery Ingng. Vol. 35, March 1958.

'Modern Vibratory Screens'. Quarry Managers Journal, 1953, 36, 2 .

'Harmonic and subharmonic response for the Duffing equation $\ddot{x}+\alpha x+\beta x^{3}=F \cos \omega t$
$(\alpha>0)$,

J. of App. Physics. Vol. 20. Nov. 1949, 1045 - 1051.

'A numerical determination of subharmonic response for the Duffing equation $\ddot{x}+\alpha x+\beta x^{3}=F \cos \omega t \quad(\alpha>0) r$. Quart. of Appl. Maths. April 1967, Vol. 25 No.I.

'An experimental investigation of forced vibrations in a mechanical system having a non-linear restoring force'. J. Appl. Phys. Vol. 17, July 1946.

'The movement of a rigid body on a horizontal plane thich is undergoing forced, nonsinusoidal motion parallel to itself'. Honours Project Report, University of Edinburgh, Engng. Dept. 1963.

'Uber neue Resonanzerscheinungen in Wechselstromkreissen'. Phys. Zeitschr. 114481910.

'Ordinary Non-Linear Differential Equations'. Z̃na Ẽã. Uxforả I956́.

'Non-Linear Oscillations'

Van Nostrand, New York, 1962.

'Asymptotic methods in the theory of nonlinear oscillations'. Translated and published by Hindustan

Publishing Corp. (Delhi) 1961.

'Conveying Speed of Vibratory Equipment'. A.S.M.E. Paper, 64-W'A/NH-I 
27. POVIDAYIO, V.A.

28. PRENDERED, J.W. and BISHOP, R.E.D.

29. RAUSCHER, M.

30. REDFORD, A.H.

31. REUTER, G.E.H.

32. RUFF, H.

33. SCHERTZ, C.E. and HAZEN, T.E.

34. STEPHEN, A.C.

35. STOKER, J.J.

36. TANIGUCHI, SAKATA, SUZUKI, OSANI

37. TRMOSHENTO, S.

38. TROITSKII, V.A.

39. TROITSKII, V.A.
'Optimum vibratory feeder operating conditions'. Machines and Tooling, Vol. XXXI, No.5, 1960.

A critical introduction to some resonance testing techniques':

Journal Mech. Eng. Sci. Vol. 5, 1963.

'Steady Oscillations of Systems with NonLinear and Unsymmetrical Elasticity?. Jour. App. Mech. Vol. 5, 1938.

'Vibratory Conveyors'. Ph.D. Thesis, Royal College of Advanced Technology, Salford, 1967.

'Subharmonics in a non-linear system with unsymmetrical restoring force? Quart. Journ. Mech. and Appl. Mech. Vol. II, Pt(2) 1949.

'Resonance Screens in Quarries'. Paper read at 1959 Annual Conf. of Inst. of Quarrying.

'Predicting Motion of a Granular Material on an Oscillating Conveyor'. Trans. Am. Soc. Agric. Engrs. Vol. 6, Nov. 1963.

Vibration of a single degree of freedom system under combined impulse and sinusoidal excitation'. N.E.L. Report No.287.

'Non-Linear Vibrations'. Interscience Publishers Inc., New York, 1950.

'Studies on Vibratory Feeders'. Bull. Jap. Soc. Mech. Eng. Vol.6, No. 2l, 1963.

!Vibration Problems in Engineering! . published by Van Nostrand, 3rd Ed. 1955.

' Variational Problems of Optimisation of Control Processes for Equations with Discontinuous Right Hand Sides'. Prikladnaia Mathematika I Mechanika 26, No. 2, 1962.

'On the Optimisation of a Vibrator Transporter Process'. Prikladnaia Mathematika I Mechanika 27, No. 6, 1963. 
40. WEHMEIER, K.H.

41. WOLFF, E.

42. KLOCKHAUS, W.

43. SCHRAUD, A.
'Vibratory Conveyors, Calculation, Design, Operation'.

Fordern und Heben, 12, 1963.

The Motion of Bulk Materials on Vibratory Conveyors'.

A.S.M.E. Paper, No. 62-WA-2I.

'Fordergeschwindigkeit von Schwingrinnen und Schwingsieben'.

Erdol und Kohle, No. 5 (1952).

'Der Reistungsbedarf von Schwingforderanlagen'. Forschung auf dem Gebiete des Ingenieurwesens No. 23, (1957). 


\section{APPENDIX A}

\section{SYSTEM COEFTIC IENTS}

The two experimental conveyors used are described in chapter 3. They are referred to as System A and System B and are similar in construction though they have different coefficients.

\section{SYSTEM A}

i) Table The table was made of mild steel. The dimensions were fixed with consideration to the available electro-magnetic vibrators. The conveying surface was 10 " long by $3^{\prime \prime}$ wide and had a ground finish. Table thickness was dictated by the method of suspension, though the centre was cut away as shown in Fig. 3.4 to reduce weight. The table complete with end caps for springs and including the force transducer and coupling screws weighed $4.7 \mathrm{lb}$.

ii) Springs From the weight of the table the spring stiffness required to give a resonant frequency in the region $8-10 \mathrm{c} / \mathrm{s}$, was calculated. Four coil springs were made up. These had a combined stiffness of $33 \mathrm{lb} . / \mathrm{in}$.

iii) Damping The damping of the system was measured both by decrement methods and by analysis of the response curve at resonance. The amplitudes of the table motion were measured using smoked glass slides. In System $A$ the guides were adjusted so that the balls were slightly loose in the Vee notches. The damping was assumed to be viscous and was .0114 of critical damping.

\section{SYSTEM B}

i) Table The table of System B was more complicated than that of System $A$ in that a pivot and clamp was added to allow the conveying 
surface to be maintained at any angle to the line of vibration. The table complete with end caps for springs and including the force transducer and coupling screws weighed $6.21 \mathrm{~b}$.

ii) Springs As for System A, four coil springs were made up. These had a combined stiffness of $47 \mathrm{lb} . / \mathrm{in}$.

iii) Damping Since this system was to be vibrated with horizontal and vertical components, no slackness was permissible in the balls and the guides were accordingly tightened to give a firm, positive location. This increased the damping of the system. In the first instance a damping factor, assuming viscous damping, was obtained as for system A. This gave damping of .053 critical. However when this value was used in an analogue computer circuit poor agreement was obtained between computed amplitudes and those found by experiment for cases in which the amplitude was small. Experiments showed that the best agreement was obtained if Coulomb damping of .16 Ib. and a viscous damping factor of .018 critical were assumed to act together.

Hard 'Impact' Springs. The same hard 'impact' springs were used in both systems. They were coil springs, one end of which was fitted with a l" radius brass keep, the other being fitted with a nounting screw. Three such springs were used. Their stiffnesses were 360 lb./in., 190 Ib./in. and $90 \mathrm{Ib} . / \mathrm{in}$.

Coefficient of friction. Previous researchers have shown that no significant difference exists between the static and kinetic coefficients of friction and further, even if the static coefficient was higher than the dynamic coefficient, little error would result if the dynamic coefficient alone was used. On such a small conveying length, errors from local variations in the coefficient of friction could be marked. 
This could be influenced by the cleanliness or degree of polish of the surface. Humid atmospheric conditions were also found to cause surface tackiness. The coefficient of fricition between the brass slider and the ground steel base was measured by tilting the base to an angle which just caused a constant slipping to occur. This value was taken as $\mu=.12$. 


\section{APPENDIX B}

THE DEVELOPMENT' OF EXPRESSTONS OF OPTIMUM TRAVEL RATE FOR A GIVEN

\section{MAXIMUM ACCELERATION}

In this section it is intended to develop solutions for the maximum travel rate which can be obtained with a given coefficient of friction $\mu$, and a maximum permissible acceleration $U$. Only harmonic solutions of period $T_{0}=2 \pi / \omega$ will be considered. The approach is from a purely physical viewpoint. Troitski ${ }^{(38)}$ has attacked the problem from a purely analytical angle as an extension of work on variational methods applied to systems of equations with discontinuous right hand sides. The extremely mathematical approach, including the choice of parameters, makes a physical interpretation of his work difficult.

There are three types of relative motion which will be considered i) Purely horizontal table motion

ii) Motion at an angle of inclination $\beta$ such that $U_{x}=U \cos \beta$ and $\mathrm{U}_{\mathrm{y}}=\mathrm{U} \sin \beta$

iii) Motion such that $U_{x}$ and $U_{y}$ are independent.

In this analysis only the conveyor surface motion is considered to be of interest, and not the mechanism by which it is produced. It does not matter whether the material being transported has a finite mass or a negligible mass. The theory of slider motion developcd in section 2 still applies.

As previously discussed there are three possible configurations of slider and table velocities

a) Single sided (forward) slip

$$
\begin{aligned}
\dot{x}(t)-\dot{X}(t) & =F(t) & & 0 \leq t \leq t_{1} \\
& =0 & & t_{I} \leq t \leq T_{0}
\end{aligned}
$$


b) Double sided slip. Sliding occurs in both directions during a cycle but with a finite period of no relative slip

$$
\begin{aligned}
x(t)-\dot{x}(t) & =F_{1}(t) & & 0 \leq t \leq t_{1} \\
& =0 & & t_{1} \leq t \leq t_{2} \\
& =F_{2}(t) & & t_{2} \leq t \leq t_{3} \\
& =0 & & t_{3} \leq t \leq T_{0}
\end{aligned}
$$

c) The slider is continuously slipping relative to the table

$$
\begin{aligned}
\dot{x}(t)-\dot{x}(t) & =F_{1}(t) & & 0 \leq t \leq t_{1} \\
& =F_{2}(t) & & t_{1} \leq t \leq T_{0}
\end{aligned}
$$

where time in the above expressions has been arbitrarily chosen to start from the instant at which slip begins or changes sign.

For periodic solutions two conditions can be stated

$$
X(t)=X\left(t+T_{0}\right) \text { and } \dot{X}(t)=\dot{X}\left(t+T_{0}\right) \quad \ldots B . I
$$

which is the condition that displacement and velocity be periodic and

$$
\int_{0}^{T_{0}} \dot{x} d t=0
$$

which is the condition that there shall be no mean velocity of the table over a cycle.

When looking for a maximum condition the rather obvious point can be made that when gradient is a liniting condition maximum areas will be bounded by straight lines at the limiting gradient rather than by curves. Thus having fixed a maximum acceleration (gradient of velocity) the maximum travel rates will result from velocity waveforms composed of straight line sections. B.I Case (I) Travel rate resulting from a purely horizontal conveyor motion. 


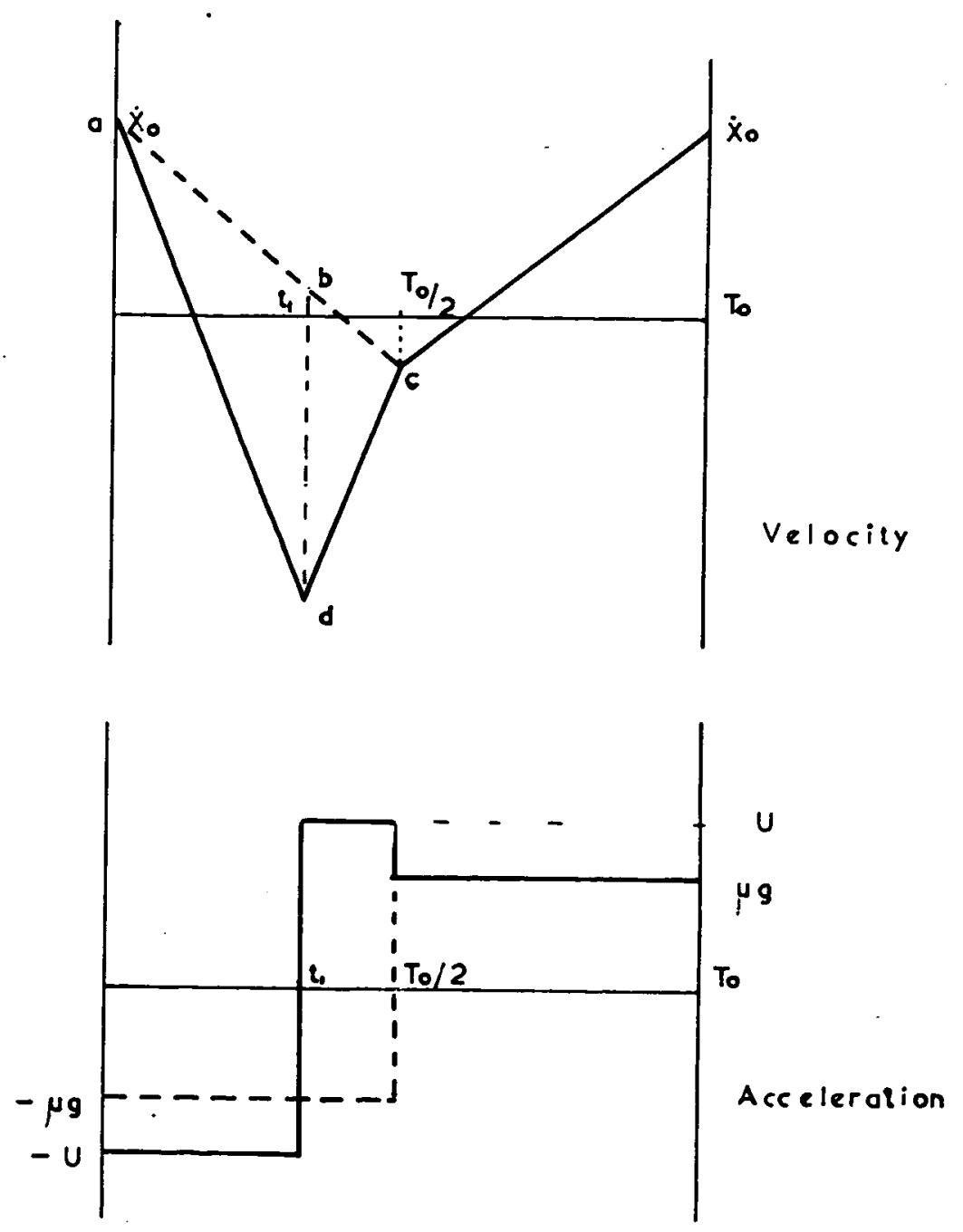

SLIDER MOTION SHOWN BY BROKEN LINE Waveform for maximum travel rote Horizontal Motion

FIG $B: I$ 
When the slider is slipping relative to the table its velocity is given by

$$
\dot{x}=\dot{x}_{0} \pm \mu g t
$$

where $\dot{x}_{0}=x_{0}$, the table velocity when slipping begins. As the discontinuity in the R.H.S. is simply a sign change, if the motion is of type (c) then $t_{1}=T_{0} / 2$ and if of type (a) $t_{1} \leq T_{0} / 2$ if the condition for a periodic solution is to apply. That is to say, positive or negative slip can extend only over half a cycle at the most.

Consider now motion of type (a)

$$
\begin{aligned}
\dot{x}(t)-\dot{X}(t) & =F(t) & & 0 \leq t \leq t_{I} \\
& =0 & & t_{1} \leq t \leq T_{0}
\end{aligned}
$$

where $t_{1} \leq T_{0} / 2$, the equality being used for a maximum. Referring to fig. B.I, let slip begin at $t=0$ when $\dot{X}=\dot{x}_{0}=\dot{x}_{0}$ Then at $t=t_{I}=T_{0} / 2 \quad \dot{x}=\dot{x}_{0}-\mu \mathrm{g}^{T_{0}} / 2$

Further, for single sided slip, which is our definition of type (a) we must have $\ddot{x} \leq \mu g \quad T_{0 / 2} \leq t \leq T_{0}$, and again the equality must be used to give a maximum.

It is now required to construct a function $X(t)$ in the zone $0 \leq \mathrm{t} \leq \mathrm{T}_{0} / 2$ to give a maximum area.

$$
\text { at } \begin{aligned}
t & =0 & & \dot{x}=\dot{x}_{0} \\
t & =T_{0} / 2 & & \dot{x}=\dot{x}_{0}-\mu \mathrm{g}^{T_{0} / 2 .}
\end{aligned}
$$

Defining $t_{2}$ such that the minimum value of $t_{2}$ occurs there gives

$$
\dot{\mathrm{x}}_{0}-u t_{2}=\dot{\mathrm{x}}_{0}-\mu \mathrm{g} \mathrm{T}_{0} / 2-u\left(\frac{\mathrm{T}_{0}}{2}-t_{2}\right)
$$

giving $\quad t_{2}=T_{0} / 4\left(1+\frac{\mu g}{\mathrm{U}}\right)$

Then the slider and conveyor velocities can be described

$$
\begin{aligned}
\dot{X} & =\dot{x}_{0}-U t & & 0 \leq t \leq T_{0} / 4\left(1+\mu \frac{\mu g}{U}\right) \\
& =\dot{x}_{0}-2 U t_{2}+U t & & \frac{T_{0}}{4}\left(1+\frac{\mu g}{U}\right) \leq t \leq \frac{T_{0}}{2} \\
& =\dot{x}_{0}-\mu g T_{0}+\mu g t & & \frac{T_{0}}{2} \leq t \leq T_{0}
\end{aligned}
$$


and

$$
\begin{aligned}
\dot{x} & =\dot{\mathrm{x}}_{0}-\mu g t & & 0 \leq t \leq \mathrm{T}_{0} / 2 \\
& =\dot{\mathrm{x}}_{0}-\mu \mathrm{gT}+\mu g t & & \mathrm{~T}_{0} / 2 \leq t \leq \mathrm{T}_{0} \quad \ldots B .7
\end{aligned}
$$

The displacement per cycle $d$ is the area abcd in Fig. Bl giving

$$
\delta=\frac{T_{0}^{2}}{16} \mid \frac{U^{2}-(\mu g)^{2}}{U}
$$$$
\text { -.. B.8 }
$$

which does not involve $\dot{X}_{0}$. This is the only unknown and can be found from condition B.2.

$$
\int_{0}^{T_{0}} \dot{x} d t=0
$$

and integrating $\dot{X}$ described in $B .6$ over the three regions gives

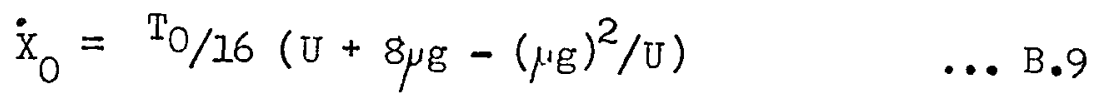

Equation $B .8$ can be shown to be the maximum possible travel rate which can occur. Considering cases (b) or (c), it can be seen that some negative slip will occur. For a periodic solution the slider velocity must change sign at $t_{1}=T_{0} / 2$ and thus table and slider velocities must be identical at this point. Thus maximising the forward slip in the region $0 \leqslant t \leqslant T_{0} / 2$ and preventing backward slip from occuring gives the maximum travel rate available under horizontal motion only.

Fig. B.I shows the acceleration of the conveyor and slider as rell as the velocities.

B.2 Case (II) Surface motion at angle of inclination $\beta^{\circ}$

In this case the table has a maximum acceleration of $U$ at an angle of $\beta$ to the horizontal such that the maximum horizontal and vertical accelerations are $U \cos \beta$ and $U \sin \beta$ respectively. Again motion of type (a) will be considered as being the most probable to give a maximum travel rate. Proceeding as before the conditions for 


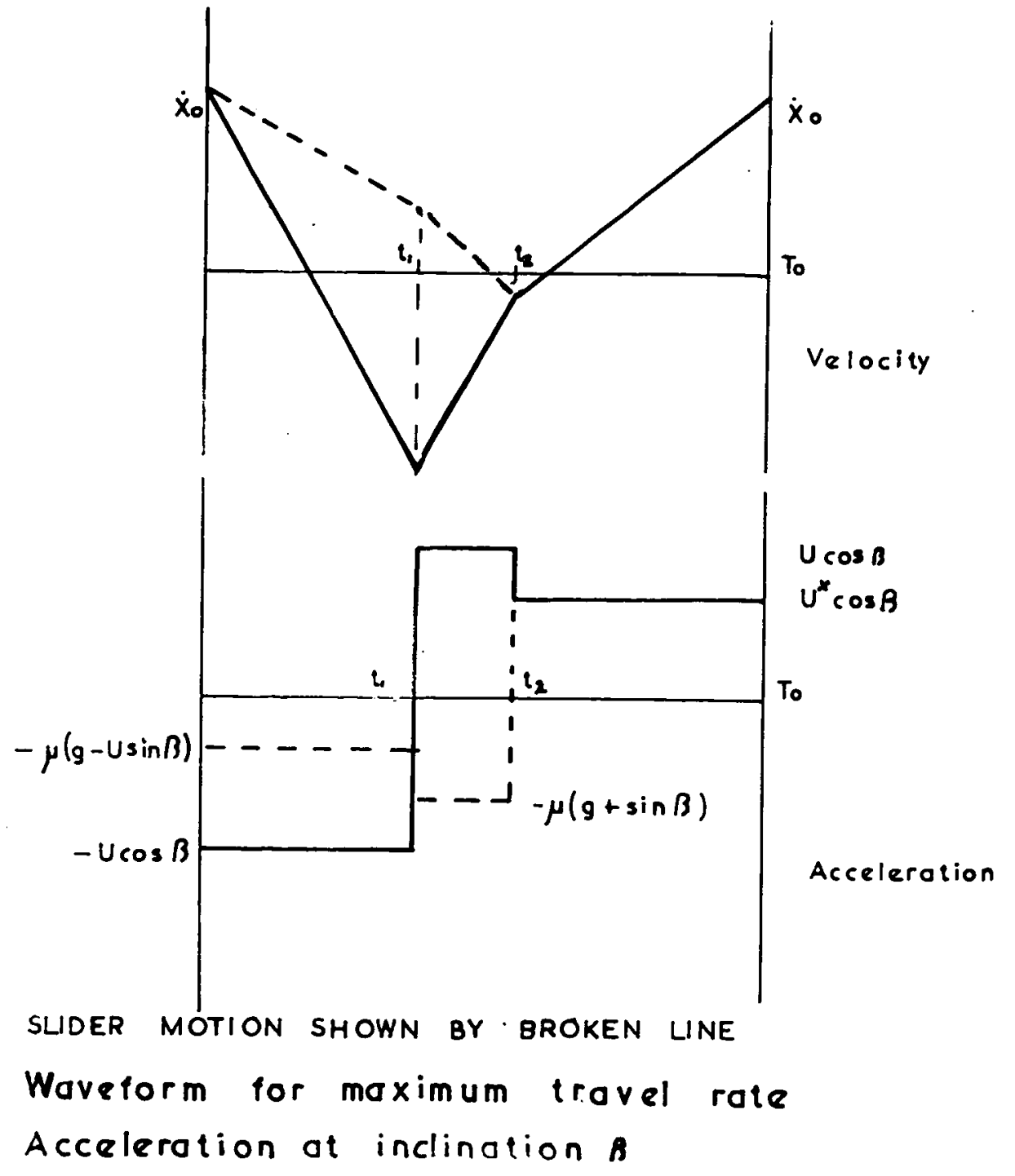

FIG $B: 2$ 
single sided slip are used.

When the material is slipping $\ddot{x}= \pm \mu(g+U(t) \sin f)$

Thus for no slip to occur the naximum acceleration which can be permitted is $U^{* 5}$ where $U^{\text {if }}$ is defined by

$$
\ddot{\mathrm{x}}=\ddot{\mathrm{x}}= \pm \mu\left(\mathrm{g}+\mathrm{U}^{\#}(\mathrm{t}) \sin \beta\right)=\mathrm{U}^{3 \pi}(\mathrm{t}) \cos \beta
$$$$
\text { ... B.10 }
$$

where $U^{F}(t) \leq U$. As before $t=0$ is defined as the instant at which slipping starts. Then the period of zero slip is $t_{2} \leq t \leq T_{0}$. Following the reasoning of the previous section Fig. B.2 is arrived at as being the best configuration.

Then

$$
\begin{aligned}
& \left.\begin{array}{l}
\dot{x}=\dot{x}_{0}-\mu(g-U \sin \beta) \cdot t \\
\dot{x}=\dot{x}_{0}-U \cos \beta \cdot t
\end{array}\right\} \quad 0 \leq t \leq t_{1} \\
& \begin{array}{l}
\dot{x}=\dot{x}_{0}-\mu(g-U \sin \beta) t_{1}-\mu(g+U \sin \beta)\left(t-t_{1}\right) \\
\dot{x}=\dot{x}_{0}-2 U \cos \beta \cdot t_{1}+U \cos \beta \cdot t
\end{array} \quad t_{1} \leq t \leq t_{2} \\
& \dot{x}=\dot{x}=\dot{X}_{0}-U^{*} \cos \beta \cdot\left(T_{0}-t_{1}\right) \quad t_{2} \leq t \leq T_{0} \quad \text {...B.II }
\end{aligned}
$$

At $t=t_{2}$ there are three conditions by which $\dot{x}=\dot{x}$ can be found.

$$
\begin{aligned}
& \dot{\mathrm{X}}\left(\mathrm{t}_{2}\right)=\dot{\mathrm{X}}_{0}-\mathrm{U}^{*} \cos F\left(\mathrm{~T}_{0}-\mathrm{t}_{2}\right) \\
& \dot{x}\left(t_{2}\right)=\dot{x}_{0}-2 U \cos \beta \cdot t_{1}+U \cos \beta \cdot t_{2} \\
& \dot{X}\left(t_{2}\right)=\dot{x}_{0}+2 \mu U \sin \beta \cdot t_{1}-\mu(g+U \sin \beta) t_{2} \quad \ldots B .12
\end{aligned}
$$

Solving these equations for $t_{1}$ and $t_{2}$ gives

and

$$
\left.t_{I}=\frac{U_{T} \frac{\pi}{2 U}}{(\mu g+U(\cos \beta+\sin \beta)}\right)
$$

$$
t_{2}=\frac{U^{m t} T(\cos \beta+\mu \sin \beta)}{\mu \mathrm{g}+U^{\pi i}(\cos \beta+\mu \sin \beta)}
$$

The relative displacement per cycle $\delta$ is found as before giving

$$
\delta=-\frac{\mathrm{T}_{0}{ }^{2}}{16 U} \frac{(\cos \beta+\mu \sin \beta)\left((\mu g)^{2}-U^{2}(\cos \hat{\beta}+\mu \sin \beta)^{2}\right)}{\cos ^{2} \beta}
$$


This is the maximum travel rate for motion of type (a), and again it is obvious that where is is possible it will give a greater travel rate than either type (b) or (c). That it does not hold over the entire range $0 \leq \beta \leq 90^{\circ}$ is obvious since when $\beta=90^{\circ}$ the denominator becomes zero, and $\delta$ becomes infinite. The above equation is valid for a maximum vertical acceleration of less than gravity and an angle $\beta$ defined with respect to $U$, the maximum acceleration, by

$$
\mu\left(\mathrm{g}+\mathrm{U}^{\#} \sin \beta\right)=\mathrm{U}^{*} \cos \beta \quad \text { where } \mathrm{U}^{*} \leq \mathrm{U} \text {. }
$$

Hence equation $B .15$ is only valid in the range $0 \leq \beta \leq \beta^{3}$ where $\beta^{\text {th }}$ is given by

$$
U^{E=}=U=\frac{\mu g}{\cos \beta^{m}-\sin \beta^{x t}}
$$

Beyond this limiting value. $\beta^{*}$ single sided slip as defined by equation (B.II) cannot occur. In this range $\beta^{\circ \pi} \leq \beta \leq 90^{\circ}$ two. modes are possible depending on whether $\mu(g+U \sin \beta)>$ or $\langle U \cos \beta$. (It is always a condition that Ucos $\beta>\mu$ (g - Usinp) for slip to occur). Fig. B.3 shows the resulting possible waveforms.

Considering firstly the case of $\beta>\beta^{*}$ and $\mu(E+U \sin p)>U \cos \beta$. This condition will lead to double sided slip. This is shown in Fig. B.3. In this case it is noted that $t=0$ is the instant of maximum table velocity and not of the start of slipping.

The conditions for perioulic solutions impiies that $\dot{x}(t)=\ddot{x}\left(t+I_{0}\right)$

$$
\dot{X}(t)=\dot{X}\left(t+T_{0}\right)
$$

The velocity of the conveyor surface is

$$
\begin{array}{rlrl}
\dot{\mathrm{X}} & =\dot{\mathrm{X}}_{0}-U \mathrm{Ut} \cos \beta & & 0 \leq t \leq \mathrm{T}_{0} / 2 \\
& =\dot{\mathrm{X}}_{0}-U \mathrm{TT} \cos \beta+U t \cos \beta & \mathrm{T}_{0} / 2 \leq t \leq \mathrm{T}_{0}
\end{array}
$$



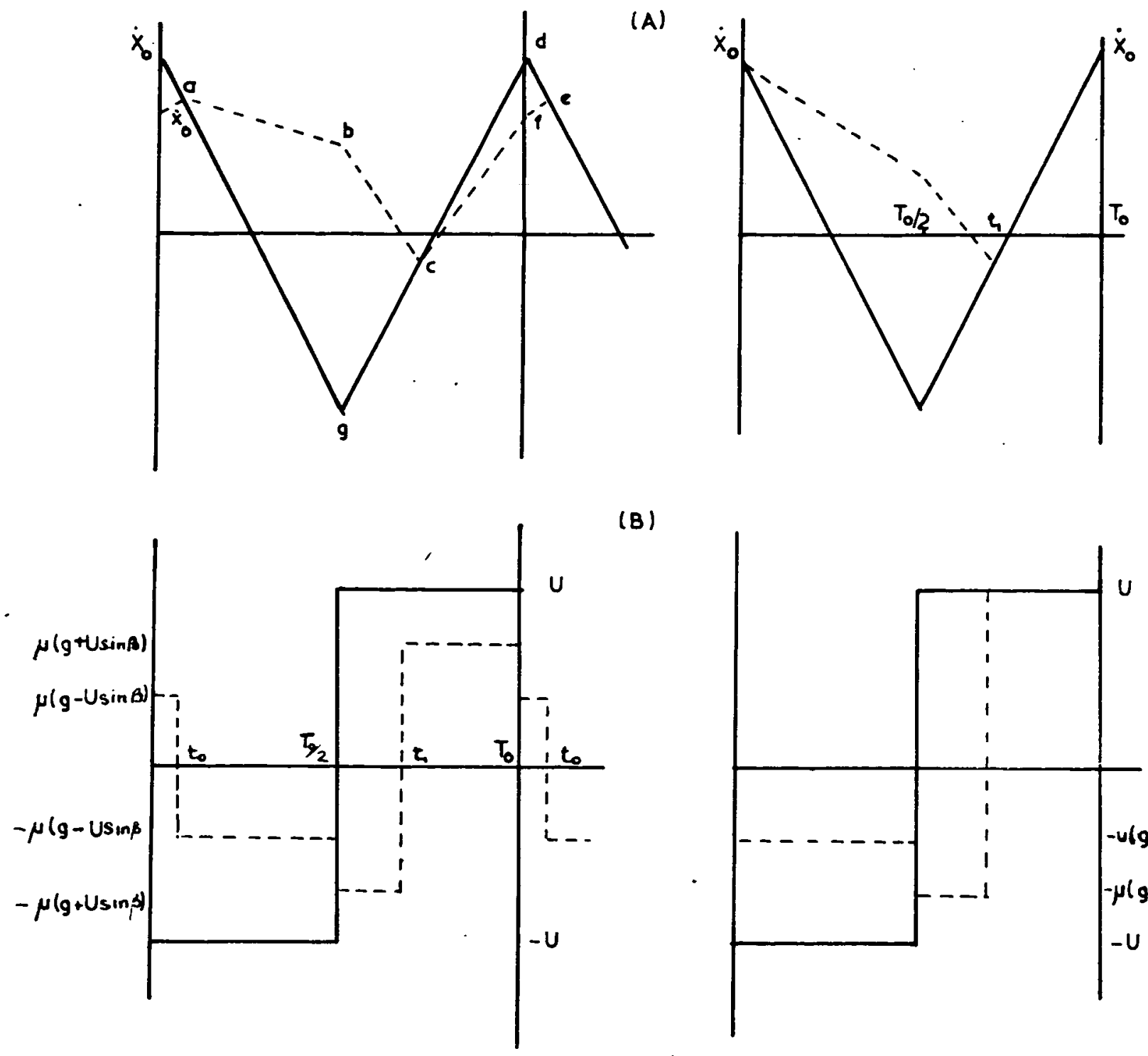

(B)
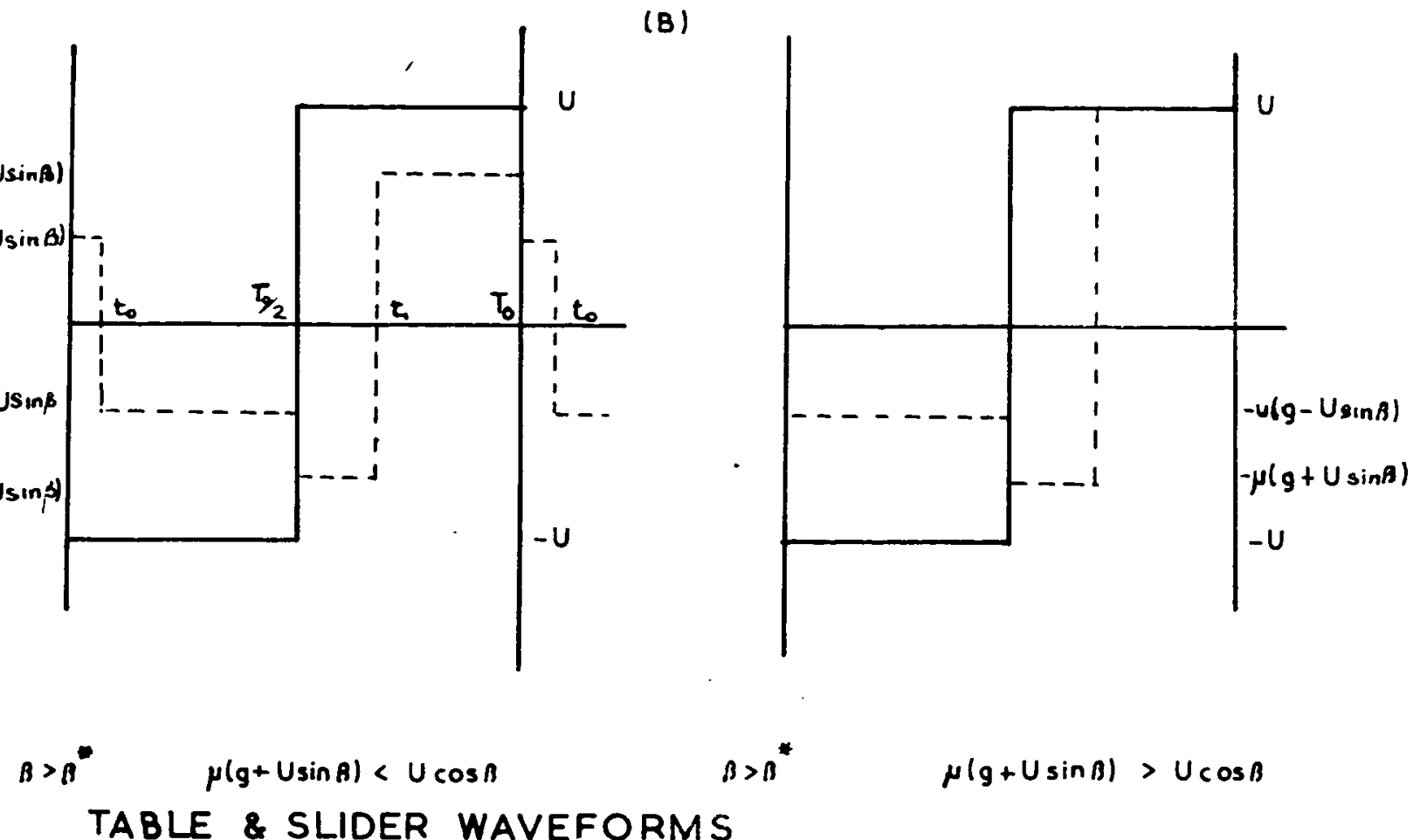

(A) VELOCITIES

(B) ACCELERATIONS

FIG. $B: 3$ 
and the slider velocity is

$\dot{\mathrm{x}}=\dot{\mathrm{x}}_{0}-\mu(g-U \sin \beta)\left(t_{0}-t\right)$

$0 \leq t \leq t_{0}$

$\dot{x}=\dot{x}_{0}-\mu(g-U \sin \beta)\left(t-t_{0}\right)$

$t_{0} \leq t \leq \mathrm{T}_{0 / 2}$

$\dot{x}=\dot{x}_{0}-\mu(g-U \sin \beta)\left(\frac{T_{0}}{2}-t_{0}\right)-\mu(g+U \sin \beta)\left(t-T_{0 / 2}\right)$

$\mathrm{T}_{0 / 2} \leq t \leq t_{1}$

$\dot{x}=\dot{x}_{0}-\mu(g-U \sin \beta) t_{0}-\mu(g+U \sin \beta)\left(T_{0}-t\right)$

$t_{1} \leq t \leq T_{0}$

... B.18

At $t=t_{I}$ there are then three equations.

$$
\begin{aligned}
& \dot{\mathrm{X}}=\dot{\mathrm{X}}_{0}-U \mathrm{UT}_{0} \cos \beta+U t_{1} \cos \beta \\
& \dot{\mathrm{X}}=\dot{\mathrm{x}}_{0}-\mu(\mathrm{g}-\mathrm{U} \sin \beta)\left(\mathrm{T}_{0} / 2-t_{0}\right)-\mu(\mathrm{g}+U \sin \beta)\left(t_{I}-{ }^{\left.\mathrm{T}_{0} / 2\right)}\right. \\
& \dot{\mathrm{X}}=\dot{\mathrm{x}}_{0}-\mu(\mathrm{g}-U \sin \beta) t_{0}-\mu(g+U \sin \beta)\left(\mathrm{T}_{0}-t_{I}\right)
\end{aligned}
$$

with the unknowns $\dot{x}_{0}, \dot{x}_{0}, \dot{x}, t_{0}, t_{1}$. Two other equations give

$$
\dot{x}_{0}=\dot{x}_{0}-U \cos \beta \cdot t_{0}
$$

and from

$$
\int_{0}^{T_{0}} \dot{X} d t=0 \quad \dot{X}_{0}=\frac{1}{4} U T_{0} \cos \beta
$$

Solution of the above equations gives

$$
t_{0}=t_{I}\left\{\frac{g+U \sin \beta}{g-U \sin \beta}\right\}-\frac{T_{0}}{2}\left\{\frac{g+2 U \sin \beta}{g-U \sin \beta}\right\}
$$

and $\quad t_{0}=T_{0}\left\{\frac{U(\cos \beta-\mu \sin \beta)-\mu g}{U(\cos \beta-\mu \sin \beta)+\mu g}\right\}-t_{I}\left\{\frac{U(\cos \beta-\sin \beta-\mu g}{U(\cos \beta-\sin \beta+\mu g}\right\}$

giving $t_{0}=\frac{T_{0}}{4 U}\left\{\frac{U(\cos \beta-\sin \beta)-\mu g}{\cos \beta}\right\}$

Then $\delta$ the relative displacement per cycle is

$\dot{\sigma}=\frac{1}{2}\left(t_{1}-t_{0}\right)(U(\cos \beta+\sin \beta)-\mu g)\left(\frac{T_{0}}{2}-t_{0}\right)-\frac{1}{2}\left(T_{0}+t_{0}-t_{1}\right)$

$$
(U(\cos \beta-\mu \sin \beta)+\mu g) t_{0}
$$

where $t_{0}$ and $t_{1}$ are found as above. This equation is valid for $\beta^{\#} \leq \beta \leq 90^{\circ}$ when $\mu(g+U \sin \dot{\mu}) \geqslant \operatorname{Ucos} F$. 
The other case which must be considered is that when $\mu(g+U \sin \beta)>U \cos \beta$. Again a waveform can be obtained (Fig. B.3). This gives

$$
\begin{aligned}
\dot{X}=\dot{X}_{0}-U t \cos \beta & 0 \leq t \leq T_{0} / 2 \\
=\dot{X}_{0}-U T_{0} \cos \beta+U t \cos \beta & T_{0 / 2} \leq t \leq T_{0} \\
& \ldots \text { B.24 }
\end{aligned}
$$

and

$$
\begin{array}{rlr}
\ddot{x} & =\dot{x}_{0}-\mu(g-U \sin \beta) \cdot t & 0 \leq t \leq T_{0} / 2 \\
& =\dot{x}_{0}-\mu(g-U \sin \beta) \cdot T_{0} / 2-\mu(g+U \sin \beta)\left(t-T_{0} / 2\right) & \frac{T_{0}}{2} \leq t \leq t_{1} \\
& =\dot{x}_{0}-U T_{0} \cos \beta+U t \cos \beta & t_{I} \leq t \leq T_{0} \\
& \ldots B .25
\end{array}
$$

at $t=t_{1}$

$$
\begin{aligned}
& \dot{\mathrm{X}}=\dot{\mathrm{X}}_{0}-\mathrm{UT} \cos \beta+U t_{1} \cos \beta \\
& \dot{\mathrm{X}}=\dot{\mathrm{X}}_{0}+U \sin \beta \cdot \mathrm{T}_{0}-\mu(\mathrm{g}+\mathrm{U} \sin \beta) t_{1}
\end{aligned}
$$

giving

$$
t_{1}=T_{0}\left\{\frac{U(\cos \beta+\sin \beta)}{U(\cos \beta+\sin \beta)+\mu g}\right\}
$$

and hence the relative displacement per cycle

$$
\delta=\mathrm{T}_{0} / 4\{\mathrm{U}(\cos \beta+\mu \sin \beta)-\mu g\} \mathrm{t}_{1}
$$

the equation being valid in the region $\beta^{25} \leq \beta \leq 90$ with $\mu(g+U \sin \beta) \leq \cos \beta$.

Equations B.15, B.23 and B.27 give the maximum travel rate which can occur for motion at an angle $\vdots$ and a maximum acceleration $U$ for a system with a coefficient of friction $\mu$. By the same reasoning as was used in the case of the purely horizontal motion it can be seen that these travel rates are indeed the best that can be achieved. Figs. B.2 and B. 3 show the velocity and acceleration of the conveyor and material in each case. These equations have been calculated for a range of 


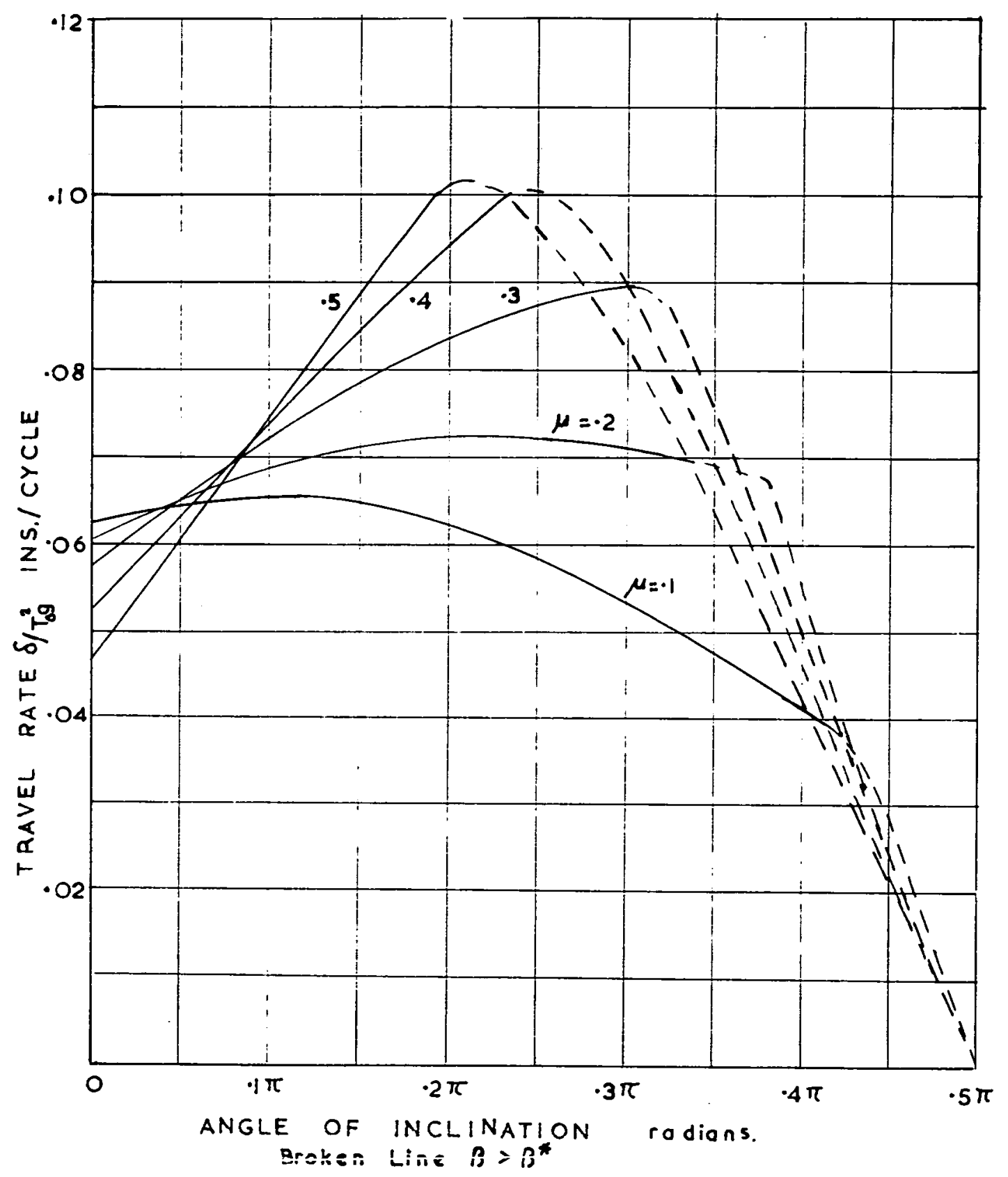

GRAPH OF MAXIMUM TRAVEL RATE Vs
ANGLE OF INCLINATION $U=9$

GRAPH B:I 


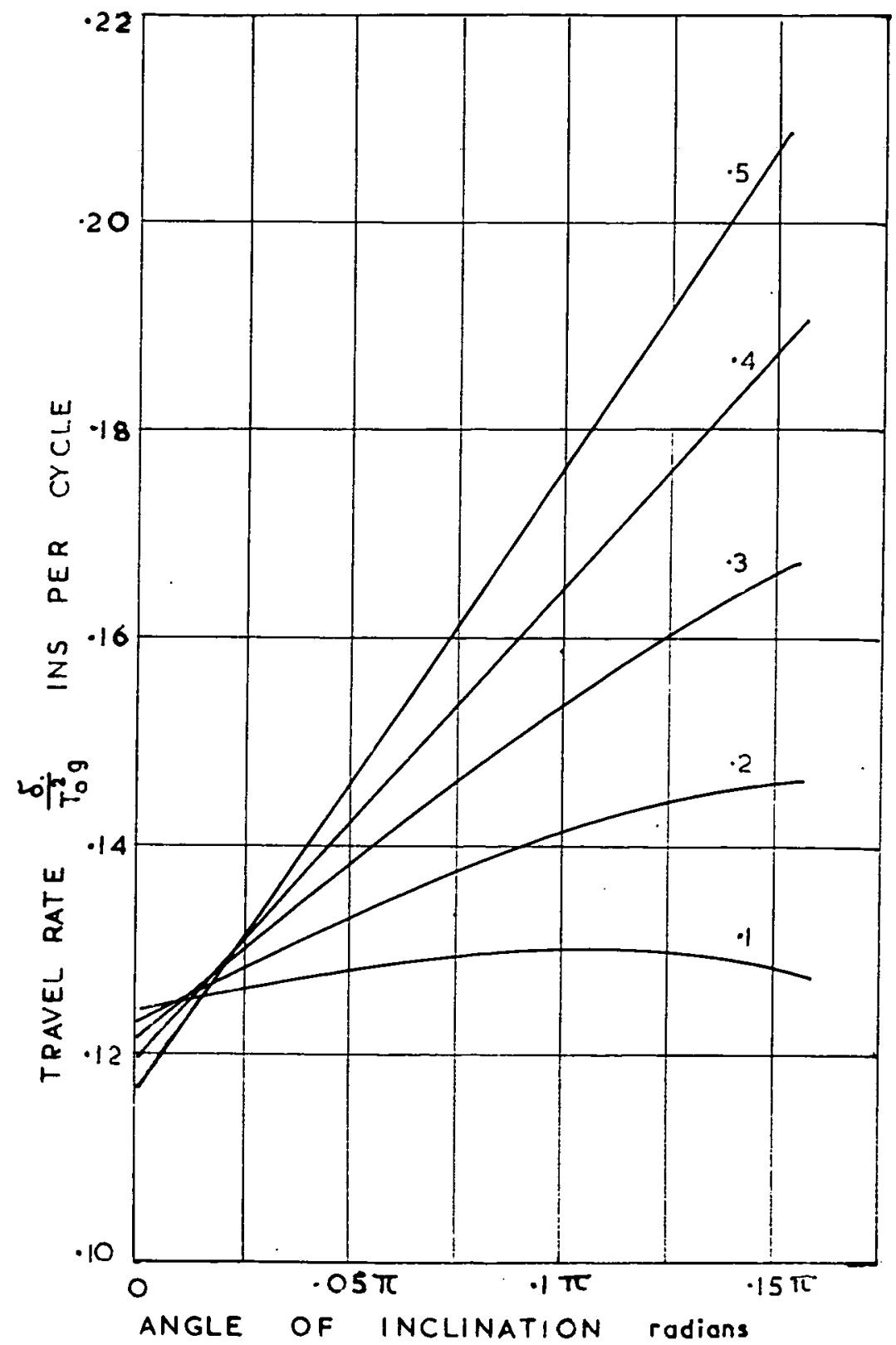

GRAPH OF MAXIMUM TRAVEL Vs ANGLE OF INCLINATION $U=2 y$

GRAPH B:2 
values $0 \leq \beta \leq 90^{\circ}$ for maximum accelerations of gravity and twice gravity for coefficients of friction $\mu=.1, .2, .3, .4$ and .5 . The calculation was carried out on a KDF9 computer programmed in Atlas Autocode. The results are shown tabulated in graphs B.I and B.2. For the case of a maximum acceleration of $2 \mathrm{~g}$ the maximum vertical acceleration exceeds $g$ at values of $\beta>30^{\circ}$ and thus sliding without throwing is no longer possible. From these graphs it is seen that while the maximum travel rate decreases with the coefficient of friction at zero slope it increases rapidly with $\mu$ as $\beta$ increases and above about $5^{\circ}$ it would appear that higher coefficients of friction are an advantage. It is only for the case of $\mu=0.1$ that the travel rate displays a turning point in the range tabulated.

For the case of maximum acceleration of $\mathrm{g}$, the maximum vertical acceleration is always less than $\mathrm{g}$ so the equations hold till $90^{\circ}$. The region $\beta>p^{3}$ is shown as a broken line. For the lower values of $\mu^{\prime}$ a turning point is reached in the region $\beta<\beta^{3}$. For $\mu=3$ and above $\beta=\beta^{5}$ is approximately the maximum value found. It is worth noting that if $\mu=1$ then $\beta^{*}=0$. Travel increases with $\mu$ about $5^{\circ}$ until $45^{\circ}$ inclination. Below $\beta=5^{\circ}$ and aboue $\beta=85^{\circ}$ it would appear that the lower values of $\mu$ give the faster transport. In the region $45^{\circ} \leq \beta \leq 85^{\circ}$ there is an optimum value of $\mu$ in range .I $\leq \mu \leq .5$.

B.3 Case (III) Motion resulting from horizontal and vertical accelerations which are independent of each other. In this case the maximurn horizontal acceleration $=U_{\mathrm{X}}$ and the maximum vertical acceleration $=U_{y} \leq \mathrm{g}$. 
The conditions for a solution to be periodic in $\mathrm{T}_{0}$ are

$$
\begin{aligned}
& \dot{X}(t)=\dot{X}\left(t+T_{0}\right) ; \quad \dot{x}(t)=\dot{x}\left(t+T_{0}\right) \\
& \int_{0}^{T_{0}} \dot{X} d t=0 \text { no mean horizontal velocity } \\
& \int_{0}^{T} \dot{Y} d t=0 \quad \text { no mean vertical velocity. }
\end{aligned}
$$

The arguments used in the previous sections may still be used, in particular it will again be assumed that the optimum travel rate will result from single sided slip. Proceeding as before, with slip beginning at $t=0$, for a given $U_{y}$ the maximum table acceleration which can occur in the horizontal plane without relative slipping is given by

$$
\ddot{x}=\ddot{x}=\mu\left(g+U_{y}\right)=U_{x}{ }^{\#} \text { where } U_{x}{ }^{*} \leqslant U_{x} \quad \ldots B .28
$$

To obtain the maximum area between the paths of table velocity and slider velocity, the slider deceleration when slipping should be as little as possible. i.e. $\ddot{x}=-\mu\left(\xi-U_{y}\right)$

However, having put this in as an optimum condition the condition of zero mean velocity over a cycle produces the result that the vertical acceleration must be given by

$$
\begin{aligned}
U_{\mathrm{y}} & =-U_{\mathrm{y}} & & 0 \leq \mathrm{t} \leq \frac{\mathrm{T}_{0}}{2} \\
& =U_{\mathrm{y}} & & T_{0} / 2 \leq \mathrm{t} \leq \mathrm{T}_{0}
\end{aligned}
$$

And thus the slider velocity is given by

$$
\begin{aligned}
& \dot{\mathrm{x}}=\dot{\mathrm{x}}_{\mathrm{O}}-\mu\left(\mathrm{g}-\mathrm{U}_{\mathrm{y}}\right) \quad 0 \leq \mathrm{t} \leqslant \mathrm{T}_{0} / 2
\end{aligned}
$$

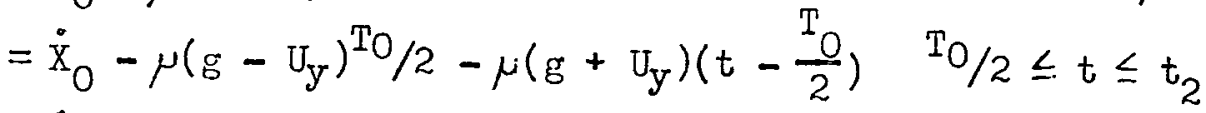

$$
\begin{aligned}
& =\dot{\mathrm{x}}_{0}+\mu\left(\mathrm{g}+\mathrm{U}_{\mathrm{y}}\right)\left(\mathrm{t}-\mathrm{T}_{0}\right) \quad \mathrm{t}_{2} \leq \mathrm{t} \leq \mathrm{T}_{0} \\
& \text {... B. } 30
\end{aligned}
$$

where $t_{2}$ is the instant at which relative slipping stops. As before the table motion must be fitted in using the maximum slopes permissible 

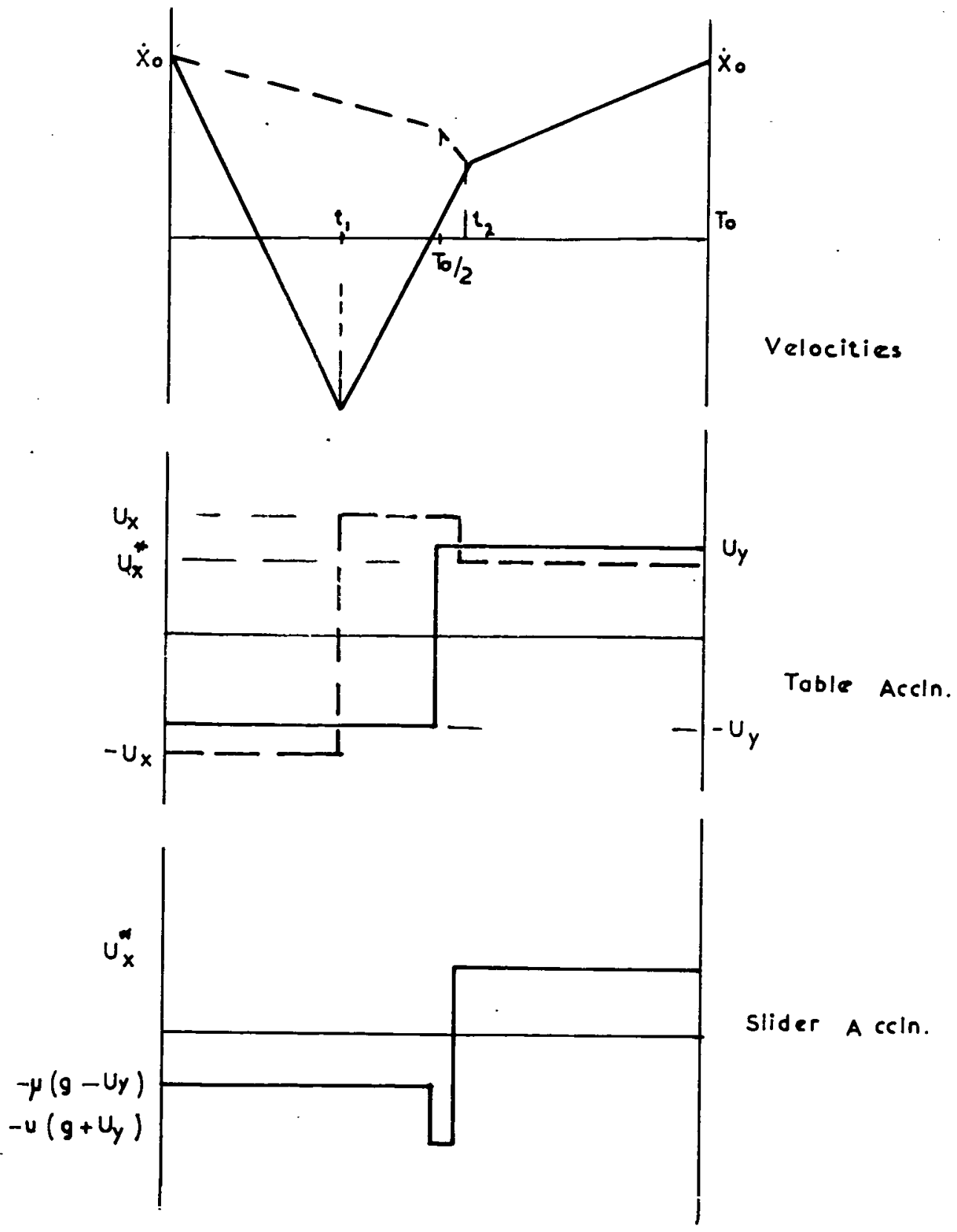

Waveform for maximum trovel rate

Table accins. horiz. \& vert. applied independently

FIG $B: 4$ 
$\pm U_{x}$ such that $\dot{x}=\dot{x}_{0}$ at $t=0$ and $\dot{x}=\dot{x}$ at $t=t_{2} . \quad t_{1}$ is defined as the point at which the minimum slider velocity occurs. Then the table velocity can be written

$$
\begin{aligned}
\dot{X} & =\dot{X}_{0}-U_{x} t & & 0 \leq t \leq t_{1} \\
& =\dot{X}_{0}-2 U_{x} t_{1}+U_{x} t & & t_{1} \leq t \leq t_{2} \\
& =\mu\left(g+U_{y}\right) t & & t_{2} \leq t \leq T_{0}
\end{aligned}
$$

Three equations for $t=t_{2}$ can be written

$$
\begin{aligned}
\dot{\mathrm{x}}=\dot{\mathrm{x}} & =\dot{\mathrm{x}}_{0}+\mu\left(\mathrm{g}+\mathrm{U}_{\mathrm{y}}\right)\left(\mathrm{t}_{2}-\mathrm{T}_{0}\right) \\
\dot{\mathrm{x}} & =\dot{\mathrm{x}}_{0}-2 \mathrm{U}_{\mathrm{x}} \mathrm{t}_{I}+\mathrm{U}_{\mathrm{x}} \mathrm{t}_{2} \\
\dot{\mathrm{x}} & =\dot{\mathrm{x}}_{0}-\mathrm{U}_{\mathrm{y} \mathrm{T}_{0}}-\mu\left(\mathrm{g}+\mathrm{U}_{\mathrm{y}}\right) \mathrm{t}_{2}
\end{aligned}
$$

from which

$$
t_{I}=\frac{T_{O}}{4}\left\{\frac{g}{g}+2 U_{y}+\frac{\mu g}{U_{y}}+\frac{U_{x}}{U_{x}}\right\}
$$

and

$$
t_{2}=\frac{T_{0}}{4}\left\{\frac{g+2 U_{y}}{g+U_{y}}\right)
$$

From these values, $\delta$ the relative displacement per cycle can be obtained, after some manipulation, in the form

$$
\delta=\frac{\mathrm{T}_{0}^{2}}{16 \mathrm{U}_{\mathrm{x}}}\left\{\mathrm{U}_{\mathrm{x}}^{2}\left(I+\frac{\mathrm{U}_{\mathrm{y}}}{\mathrm{g}+\mathrm{U}_{\mathrm{y}}}\right)^{2}-(\mu \mathrm{g})^{2}+\frac{4 \mu \mathrm{U}_{\mathrm{x}} U_{\mathrm{y}}^{2}}{\mathrm{~g}+\mathrm{U}_{\mathrm{y}}}\right\} \quad \ldots \mathrm{B} .35
$$

By the same intuitive approach as used in the previous cases this system is the best travel rate that can occur. The slider and table velncity and acceleratiuns are piotted in Fig. B.4.

B.4

Expressions have thus been derived for each of the three cases by a consideration of the physical features of the problem. As a check it can be shown that equation (B.15) reduces to $(B .8)$ if $\beta=0$. Similarly $(B .35)$ reduces to $(B .8)$ if $U_{y}=0$. These equations are similar to those found by Troitski who derived equations (B.8) and 
(B.35). He does however give a slightly different answer from (B.15) namely

$$
\delta=-\frac{\mathrm{T}_{0}^{2}}{16 U}\left\{\frac{(\cos \beta+\mu \sin \beta)\left((\mu g)^{2}-U^{2}(\cos \beta+\mu \sin \hat{\rho})\right)}{\cos ^{2} \beta}\right\}
$$

The close similarity leads to the possibility of printers errors, as other minor mistakes occur in the paper. Troitski does not develop case (II) beyond equation (B.15).

Considering the example $U=3.6 \mathrm{~g} ; \beta=30^{\circ} ; \mu=0.5 ; \quad U_{x}=3.12 \mu \mathrm{g}$; $\mathrm{U}_{\mathrm{y}}=1.8 \mu \mathrm{g}$ i.e. the maximum table acceleration is the same in all three cases, we get

$$
\begin{aligned}
\delta_{\text {horizontal }} & =.208 \mu \mathrm{gT}_{\mathrm{O}}^{2} \\
\delta_{\beta} & =.396 \mu \mathrm{gT}_{0}^{2} \\
\delta_{\text {independent }} & =.540 \mu \mathrm{gT}_{0}^{2}
\end{aligned}
$$

For the same case Troitoki gives

$$
\begin{aligned}
\delta_{\text {horizontal }} & =.208 \mu \mathrm{gT}_{0}^{2} \\
\delta_{\beta} & =.418 \mu \mathrm{gT}_{0}^{2} \\
\delta_{\text {independent }} & =.550 \mu \mathrm{gT}_{0}^{2} .
\end{aligned}
$$

This approach allows accelerations to be discontinuous - finite, instantaneous changes taking place. In a practical system this is not likely to be achieved. The maximum acceleration occurs over prolonged periods giving rise to large table velocities and fast travel rates. If table acceleration is an important design criterion, and in considering table frame stresses this is likely to be so, then these travel rates would be an important basis on which to evaluate the efficiency of various waveforms.

The example calculated above shows what is likely to be a standard pattern. Greater efficiency results from the presence of vertical acceleration. When the horizontal and vertical accelerations 
are components of one acceleration at an inclination $\beta$ the optimum travel rate is about twice that which can be achieved under horizontal motion alone. When the two accelerations are independently applied, but still of the same period a further $35 \%$ increase results.

A set of results were obtained by analogue computer for comparison with these optimum travel rates with a view to comparing the efficiencies of waveforms. However there is no, basis on which to evaluate these waveforms on the basis of the spring characteristic from which they were obtained as the response depends on the resonance characteristic and the frequency of excitation involved. The computed results are for System $B$ for a coefficient of friction of 0.12 and a maximum acceleration of $\mathrm{g}($ or $-\mathrm{g})$. The frequency was $9 \mathrm{c} / \mathrm{s}$. The absolute maxima for this system are for motion horizontal, $\delta=.292$ inches/cycle and for motion at $30^{\circ}, \delta=.318$ inches/cycle.

TABLE BI

EFFICIENCY OF WAVEFORMS. SYSTEM B. $U=g ; \mu=.12 ; \mathrm{f}=9 \mathrm{c} / \mathrm{s}$.

1)

\begin{tabular}{|c|c|c|c|c|c|c|}
\hline \multirow[b]{2}{*}{$\mathrm{K}_{1}$} & \multirow[b]{2}{*}{$\mathrm{K}_{2}$} & \multirow[b]{2}{*}{ gap } & \multicolumn{2}{|c|}{ HORIZONTAL } & \multicolumn{2}{|c|}{ INUCLINATION $30^{\circ}$} \\
\hline & & & $\delta$ & 7 & $\delta$ & 7 \\
\hline 47 & 0 & LINEAR & 0 & 0 & .0961 & 30.2 \\
\hline 47 & 360 & 0 & .0646 & 22.1 & .0704 & 22.1 \\
\hline$"$ & " & .025 & .0653 & 22.4 & .0869 & 27.3 \\
\hline$"$ & $"$ & .050 & .0388 & 13.3 & .0921 & 29.0 \\
\hline 47 & 190 & 0 & .0866 & 29.7 & .1140 & 35.8 \\
\hline$"$ & $"$ & .025 & .0570 & 19.5 & .1018 & 32.0 \\
\hline$"$ & $"$ & .050 & .0342 & 11.7 & .0940 & 25.5 \\
\hline 47 & 90 & 0 & .0530 & 18.1 & .1116 & 35.0 \\
\hline$"$ & $"$ & .025 & .0419 & 14.3 & .0998 & 31.2 \\
\hline$"$ & $"$ & .050 & .0260 & 8.9 & .0952 & 30.0 \\
\hline
\end{tabular}


The maximum efficiency found above is approximately $30 \%$ for horizontal motion and $35 \%$ when the motion is at an angle $\beta=30^{\circ}$. The linear system does not, of course, have a travel rate for purely horizontal motion. The system with the least non-linearity (47/90/050) shows the lowest travel rate for horizontal motion and the greatest improvement when a vertical component is introduced as might be expected. Values not shown in the table indicate that with $\beta=60^{\circ}$ the linear case has an efficiency of about $55 \%$. As has previously been mentioned, too much cannot be read into the values of table Bl. The spring configuration on its own is no absolute reference to the waveform, which is a function of many parameters associated with the non-linear resonance.

It has been shown that the greatest travel rates will be obtained from a system whose horizontal and vertical accelerations are applied independently. While until recently all work on vibratory conveyors has been with horizontal and vertical components 'in-phase' as in section B.2, Redford $(30)$ examined the possibilities of incorporating a phase shift between the horizontal and vertical components. Using sinusoidal waveforms he showed that this could produce more favourable travel rates. Despite the fact that the results quoted were for throwing with a maximum vertical acceleration of $1.2 \mathrm{~g}$ a comparison was attempted between Redford's work and the results predicted by section B.3. Taking the case when the conveying surface is horizontal with a maximum vertical acceleration of $1.2 \mathrm{~g}$, an angle of vibration of $20^{\circ}$ and a coefficient of friction $\mu=.2$, Redford found that the travel rate would be a maximum if the vertical motion lagged behind the horizontal motion by $35^{\circ}$. Using these parameters with $\mathrm{U}_{\mathrm{y}}=\mathrm{g}(\mathrm{c} / \mathrm{f}$ Redford $1.2 \mathrm{~g})$, 


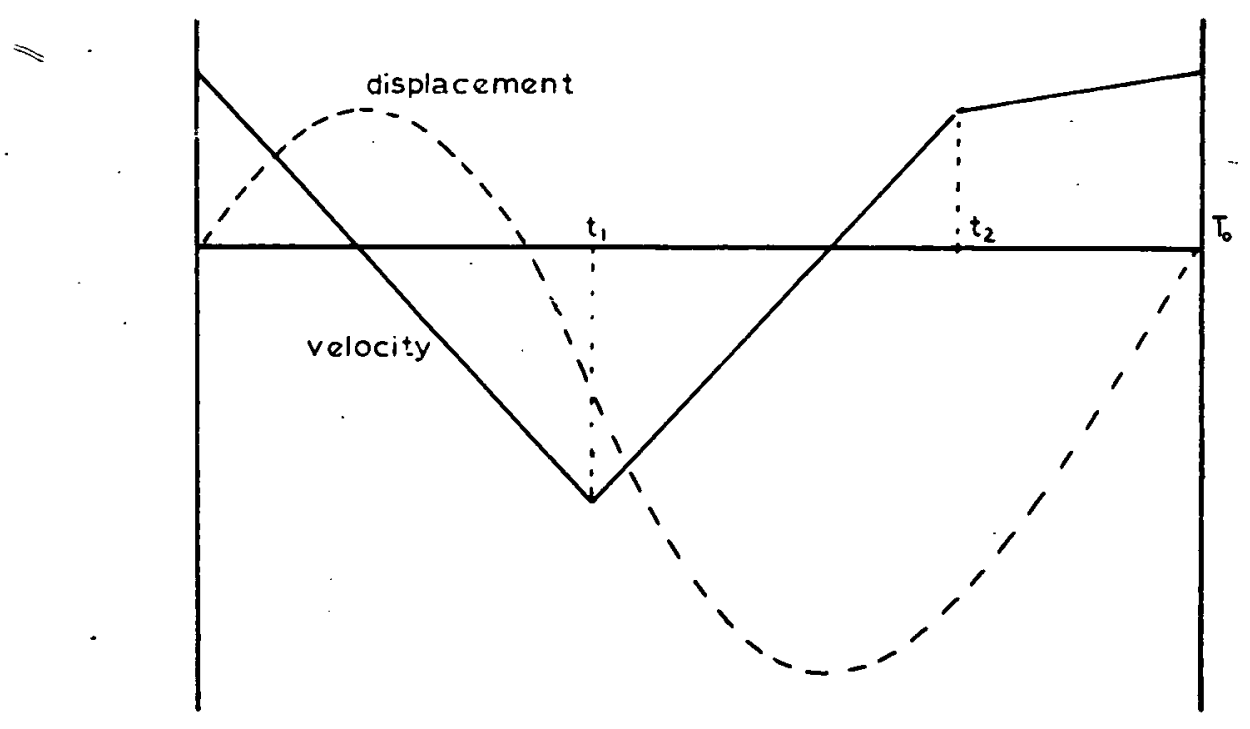

HORIZONAL MORON.

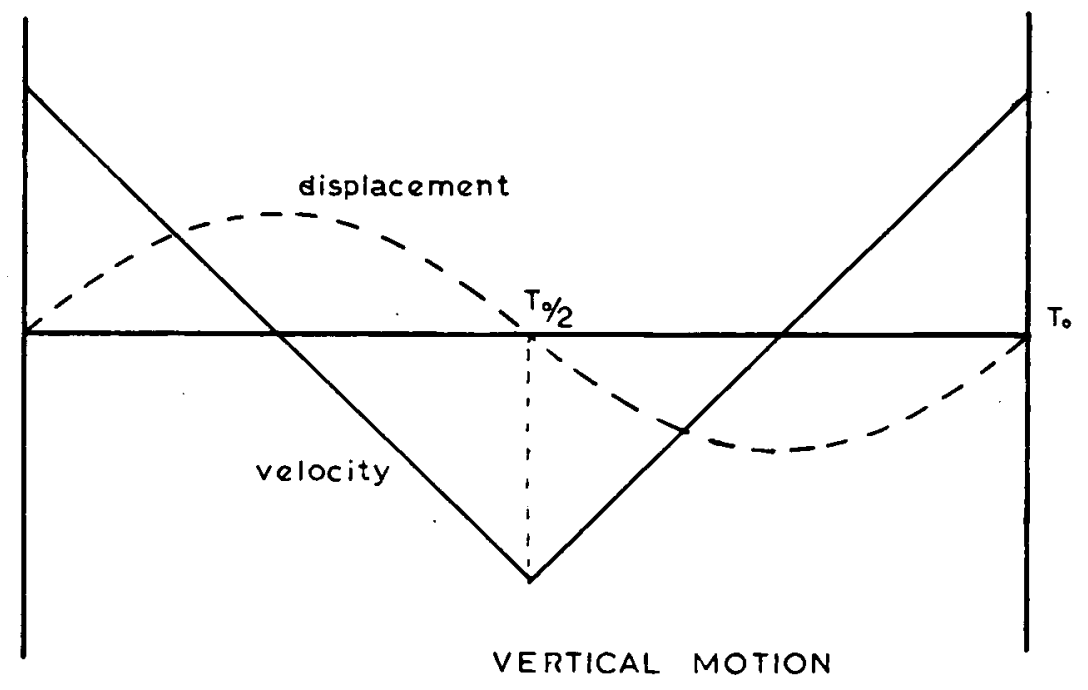

HORIZONTAL \& VERTICAL VELOCITY \& DISPLACEMENT WAVEFORMS TO GIVE MAXIMUM TRAVEL RATE WHEN COMPONENTS ARE APPLIED INDEPENDENTLY.

FIG. B:5. 
$\mathrm{U}_{\mathrm{X}}=\mathrm{g} / \tan 20^{\circ}=2.75 \mathrm{~g}, \mathrm{U}_{\mathrm{x}}^{\mathrm{*}}=.4 \mathrm{~g}$, section $\mathrm{B} .3$ gave $\mathrm{t}_{1}=.393 \mathrm{~T}_{0}$ and $t_{2}=.75 \mathrm{~T}_{0^{\circ}}$ Then representing the waveforms $\mathrm{U}_{\mathrm{x}}$ and $\mathrm{U}_{\mathrm{y}}$ in Fig. $\mathrm{B} .4$ by a Fourier series, and considering only the fundamental term, harmonic analysis gave $U_{x}=R \sin \left(t+220^{\circ}\right)$ while by inspection $U_{y}=S \sin \left(t+180^{\circ}\right)$. This gives an almost identical result to that found by Redford.

Another point which emerges from the analysis is that the result quoted by Redford that travel rate (inches/second) is inversely proportional to the frequency, is show in the various formulae. The optimum travel rate will thus improve as frequency decreases. For a given maximum acceleration this would produce larger velocities and displacements which would probably be limited by physical considerations. This indicates that shaker conveyors have a higher 'waveform efficiency' than is likely to be found with vibratory conveyors. Fig. B.5 shows velocity and displacement waveforms for the case calculated above. The vertical displacement is composed of two parabolic curves. While this may seem very similar to a sinusoidal waveform the latter would. produce poor approximation to the acceleration curve required. Methods of obtaining the desired curves by non-linear spring characteristics would prove interesting.

It should be understood that this Appendix allows the development of expressions for 'waveform efficiency'. This is similar to the work done by Redford. The fact that the expressions are in terms of maximum acceleration may appear very attractive at first sight. This approach reflects badly on the non-linear system used in this thesis, for peak acceleration is of very short duration. It should be realised that the 'waveform efficiency' may not necessarily be the same as efficiency of the conveyor system. Large peak accelerations are obtained in the non-linear system with low power input. If a 
new criterion of 'root mean square acceleration' was used instead of maximum acceleration then there is little doubt that the efficiency of the non-linear system would exceed that of a linear system. Analogue computer investigation of the results of this Appendix could be conveniently carried out using the circuit developed in chapter 4 to generate the slider motion. 


\section{APPENDIX $\quad \mathrm{C}$}

\section{EXPERTMENTS WITH SAND}

A short investigation was carried out into the behaviour of sand undergoing vibratory transport. The behaviour of sand and similar bulk meterials under dynamic conditions is a largely unexplored field. The following paragraphs describe some very elementary exploratory studies.

The sand may be assumed incompressible and composed of layers between which there is a constant coefficient of friction $\mu_{\mathrm{s}}$ throughout the depth, with a coefficient of friction $\mu_{f}$ between the sand and the conveyor surface. If the conveyor is vibrating with displacement $X(t)$ in the horizontal plane, the sand will move as a rigid body if $\mu_{f}\left\langle\mu^{\prime} s^{*}\right.$ If $\mu_{f}>\mu_{s}$ some slipping between the layers might be expected. Due to the sidewall effects there will also be a frictional force $\mu_{f} R$ where $R$ is the integral of the normal pressure. This sidewall pressure distribution has been found to be approximately hydrostatic $(2)$, hence $R=\rho g h^{2}$ where $h$ is the depth and $\rho$ the density of sand. Then for rigid body motion, the material displacement $x(t)$ is given by

$$
M \ddot{x}= \pm \mu\left(M g+g h^{2}\right)
$$

during slipping, which begins if $|\ddot{X}|>\mu \cdot g$ and continues until $x=x$. If slipping between layers is assumed to take place, equating the horizontal forces on a small element of mass $m$ at depth $h$, ignoring sidewall effects

$$
\begin{gathered}
p \ddot{x} d h= \pm \mu g(p h-p(h+d h)) \\
\text { or } \quad \ddot{x}= \pm \mu g
\end{gathered}
$$$$
\text { -.. G.2 }
$$

which is the same equation as for rigid body motion. This indicates that slipping between layers will not occur, and the sand moves as a single rigid body. 


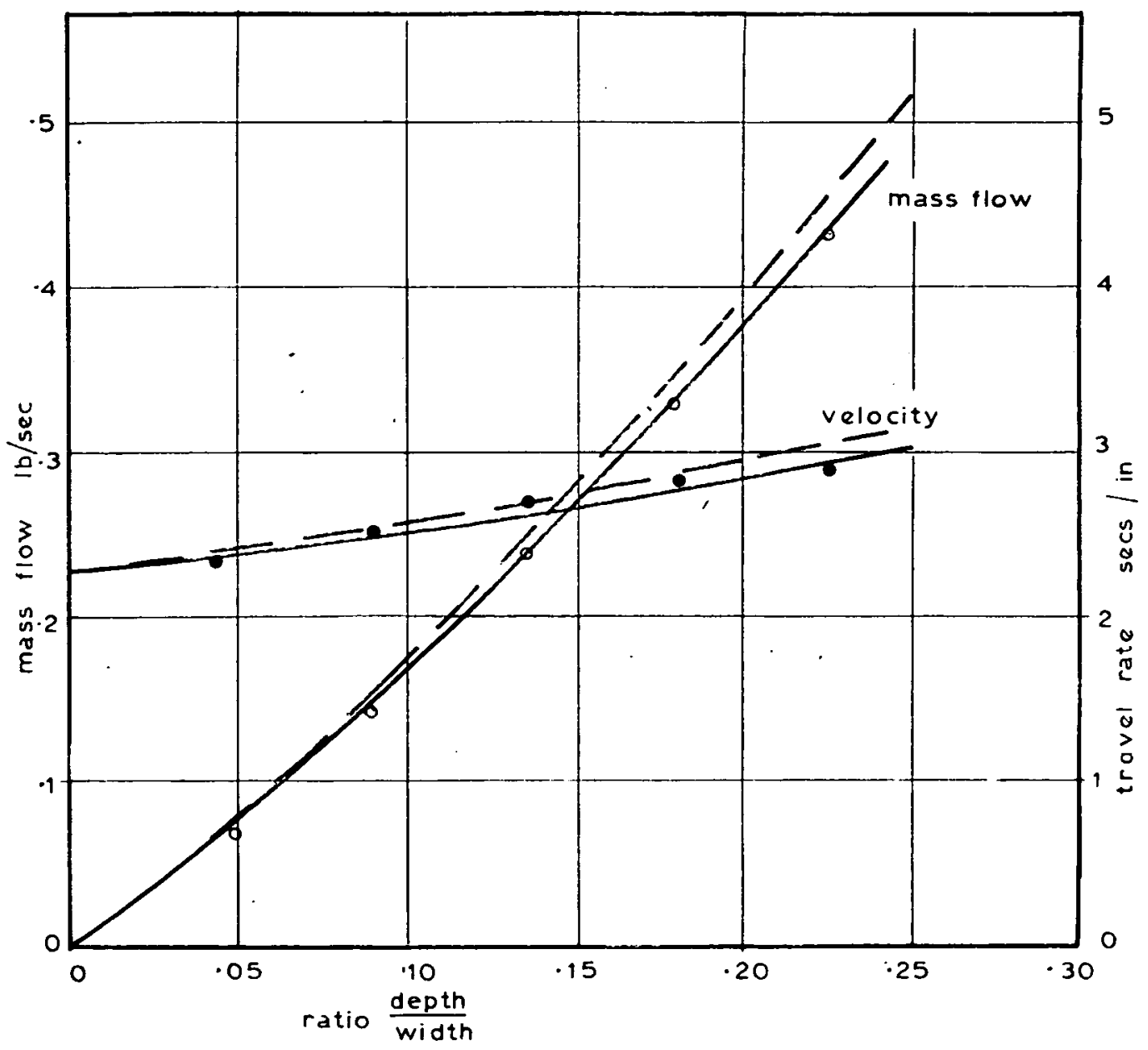

full line - experimental

broken line - theoretical

EFFECT OF CHANGE IN DEPTH ON MATERIAL VELOCITY \& MASS FLOW RATE.

FIG. $C: 3$

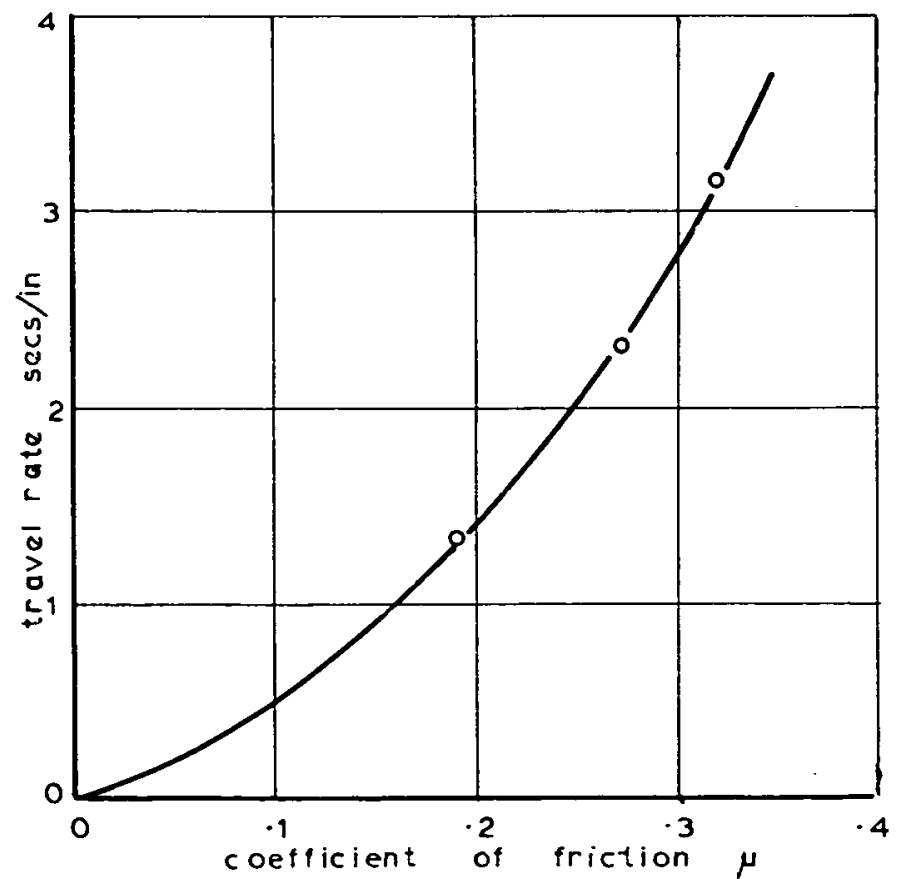

Sinusoidal Motion $\beta 30^{\circ}$ Amplitude .190 in

Frequency $8.5 \mathrm{c} / \mathrm{s}$

. VARIATION IN TRAVEL RATE WITH $\mu$. FIG C: 4 
A more general approach is to allow both the density and the coefficient of friction $\mu$ to be linear functions of depth $h$.

$$
\text { Let } \begin{aligned}
\mu(\mathrm{h}) & =\mu_{0}+\mu_{\mathrm{s}} \cdot \mathrm{h} \\
\mu(\mathrm{h}) & =\mu_{0}+\mu_{\mathrm{s}} \cdot \mathrm{h}
\end{aligned}
$$

Equating forces on an element of unit width at depth $h$, with the layers above and below slipping relative to it with velocities of opposite sign gives $\quad P(h) \cdot d h \cdot x= \pm g\left(\mu(h) M_{1}-\mu(h+d h) M_{2}\right) \quad \ldots c .4$ where

$$
\begin{aligned}
& M_{1}=\int_{0}^{h} \rho(h) d h=\rho_{0} h+\frac{1}{2} \rho_{s} \cdot h^{2} \\
& M_{2}=\int_{0}^{h+d h} \rho(h) d h=P_{0}(h+d h)+\frac{1}{2} \rho_{s}(h+d h)^{2}
\end{aligned}
$$

Substituting into $\mathrm{C.4}$ and expanding gives

$$
\ddot{x}= \pm g\left\{\mu(h)+h \frac{d \mu}{d h} \cdot\left(\rho_{0}+\frac{1}{2} \rho_{s} h\right) /\left(\rho_{0}+p_{s} h\right)\right\}
$$

and this gives

$$
\frac{d \dot{x}}{d h}= \pm \mu_{s} g\left\{2-\rho_{s} \cdot h\left(P_{0}+\frac{I}{2} \rho_{s} h\right) /\left(\rho_{0}+\rho_{s} h\right)^{2}\right\} t+\dot{x}_{0} \quad \ldots c .5
$$

where $\frac{d \dot{x}}{d h}$ is the velocity gradient.

An example calculated with $P_{\mathrm{s}} \mathrm{d}=.2 P_{0}$ and $\mu_{\mathrm{s}} \mathrm{d}=.2 \mu_{0}$ showed an almost linear relationship for the velocity gradient with the bottom layer being decelerated faster than the top layers. Reference to Fig. C.2 shows that if single sided slip is occurring, and all the layers begin to slip relative to each other simultaneously, then surface layers will travel faster than bottom layers. If, however, continuous slip occurs, reference to the figure shows that the gain made in forward slipping is lost in backward slipping. A time lag is introduced between the relative motion of layers, but no nett change in travel rate occurs. 

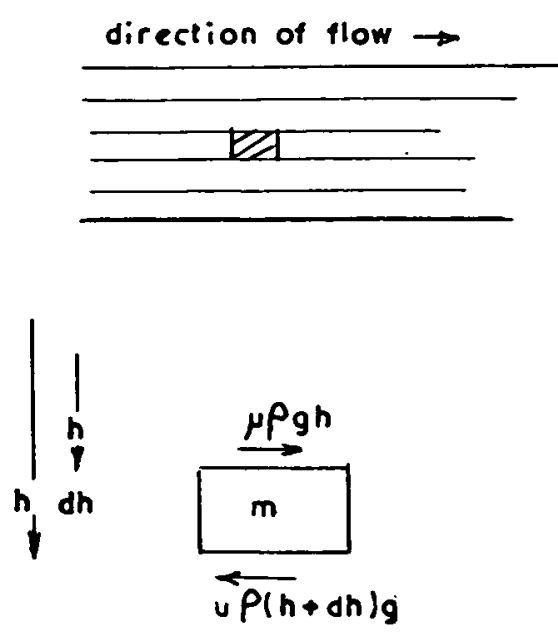

Forces on Element
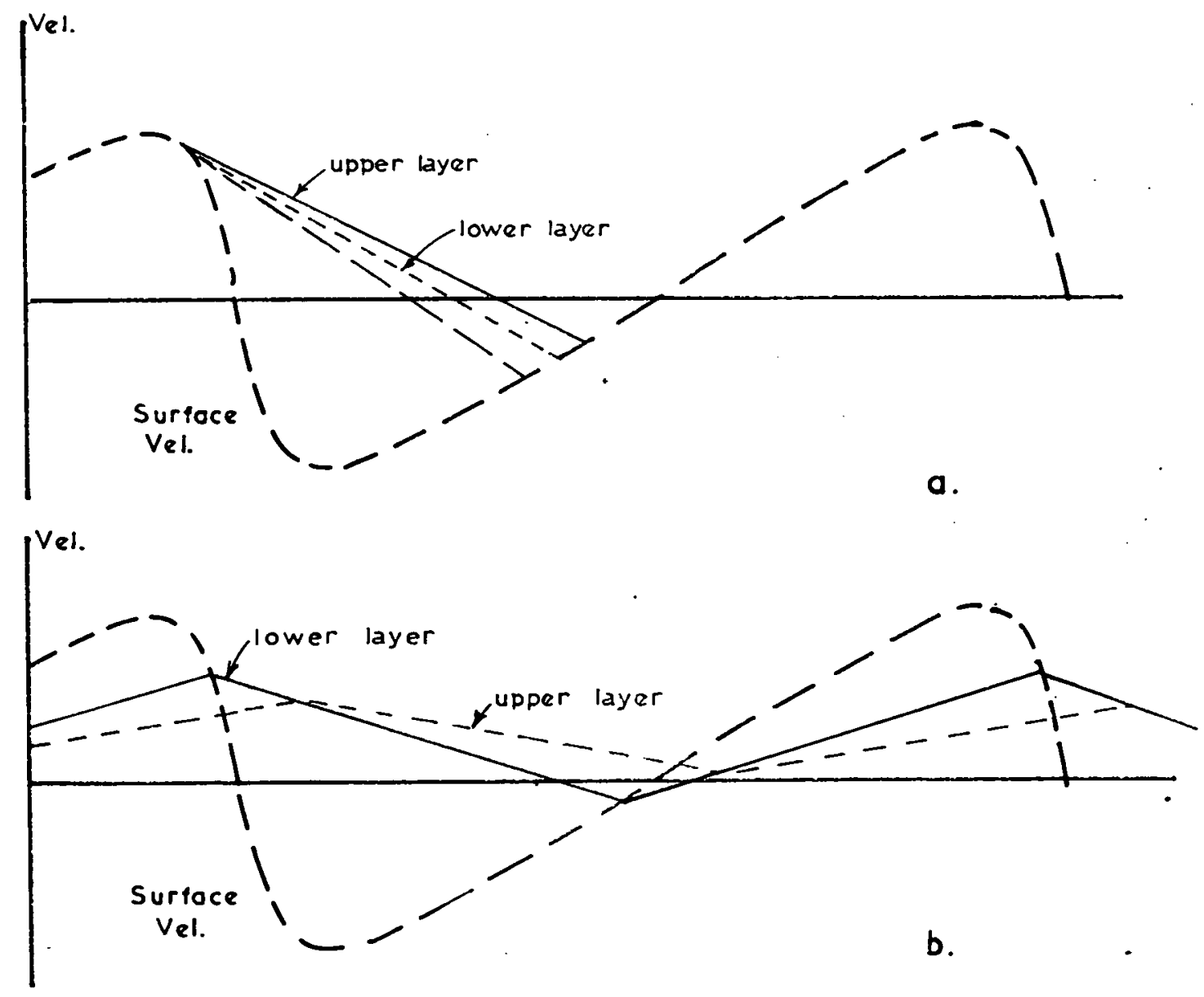

Effect of relative slipping of layers 
Various experiments were used to establish whether or not a velocity gradient exists in a bulk material being transported by vibration The most simple model used was a pack of playing cards. Since any relative movement of a card affects the balance and thus the forces acting between layers, no conclusive results were obtained.

A perspex trough was constructed and fitted onto the vibrating table used for the experiments with the brass slider. This was almost identical to the trough shown in Fig. 2.I a with a gate to control the material depth. With the system vibrating, sand was poured into the hopper. It travelled along the conveyor and was collected at the other end. When steady conditions were established the vibration was stopped and traces inserted into the sand to indicate the displacement at various depths. These tracers were either small paper tabs, or columns of white powder inserted down into the sand against the perspex sidewall. The conveyor was then vibrated for several cycles when the new position of the indicators could be studied. It was found that with the exception of the surface layer, where some irregular rolling of particles occurred, the sand moved as a rigid body.

A further experiment was carried out keeping a constant amplitude of vibration and measuring the travel rates for various depths of material. The depth was measured slightly 'downstream' from the gate, and the mass flow was measured by collecting the sand passed by the conveyor over a known time interval. The mass flow rates are shown in Fig. C.3, together with a material velocity rate calculated from the mass flow. Under identical conditions of amplitude and frequency, values were obtained of the travel rate of sliders having various coeffieicnts of friction. This is shown in Fig. C.4. The projection of the velocity curve on Fig. C.3 to the vertical axis gives a travel rate for the 
material with zero depth. From this a value of the coefficient of friction of sand on perspex with no sidewall effect can be obtained by reference to Fig. C.4. If the sand moves as a rigid body, then from equation $\mathrm{C} .1$ the friction force opposing relative motion is $\pm \mu\left(\mathrm{Mg}+\rho \mathrm{gh}^{2}\right)$. Writing this as $\pm \mu^{\prime} g$ gives

$$
\mu^{\prime}=\mu\left(1+\frac{h}{b}\right)
$$

where $\mu^{\prime}$ is the effective coefficient of friction assuming rigid body motion and hydrostatic distribution of sidewall forces. Travel rates for various depths assuming this variation in $\mu^{\prime}$ are shown in Fig. C.3, the agreement between this and the experimental curve showing the validity of the assumptions. Attempts to find a value of $\mu$ for sand and perspex by pulling a bottomless can of sand over a perspex surface did not give the same value so that obtained above, but a somewhat higher one. The difference may be between actual sliding of sand on perspex and shearing between two layers of sand.

The above work is at a very elementary level. It appears that rigid body motion is at least a good approximation to what actually happens. Very fast travel rates can be obtained with a non-linear system vibrating in a purely horizontal motion. No one who has seen this could fail to notice a similarity between the flow of fine sand and liquid flow, and a further investigation of this would be interesting. In general a vibratory conveyor for sand would not be horizontal but have a vertical component, possibly enough to cause throwing. This would be necessary to minimise the abrasive effects of the sand slipping. 


\section{APPENDIX D}

THEORY OF MOTION WHEN CENTRE OF GRAVITY IS NOT AT GEOMETRIC CENTRE

In the nature of surfaces, contact between two smooth flat surfaces occurs at a number of high spots, and not by a uniform contact over the entire base area. The brass sliders used in the experiments were turned on a lathe. It was soon realised that the centre was a likely high-spot, and it was accordingly recessed. However, despite this, a brass slider with a centre which was slightly recessed still exhibited some 'directional preference'. In its most marked form this meant that if the table was executing simple harmonic motion in a purely horizontal plane, at certain angular attitudes of the slider, transport along the table could occur, which is contrary to Fig. 2.4a. This effect could be accentuated by adding an additional mass to offset the centre of gravity.

Considering a body whose centre of gravity is not central, such as is shown in Fig. D.Ia, and whose contact with the surface is a simple 'two point' contact.

Then

$$
\begin{aligned}
m \ddot{x} & =-\mu\left(R_{1}+R_{2}\right) \\
m g & =R_{1}+R_{2} \\
R_{1} a & =R_{2} b+\mu\left(R_{1}+R_{2}\right) h
\end{aligned}
$$$$
\text { ... D.I }
$$

while slipping is taking place.

Hence $\quad R_{I}=\frac{m g(b+\mu h)}{a+b} ; R_{2}=\frac{m g(a-\mu h)}{a+b} \quad \ldots D .2$

Since $R_{1} \geqslant 0$ and $R_{2} \geqslant 0, \mu h \leq a$ otherwise the body will pivot about $R_{1}$. If this happens, using the convention of Fig. D.Ia, when the body is slipping and tilting 

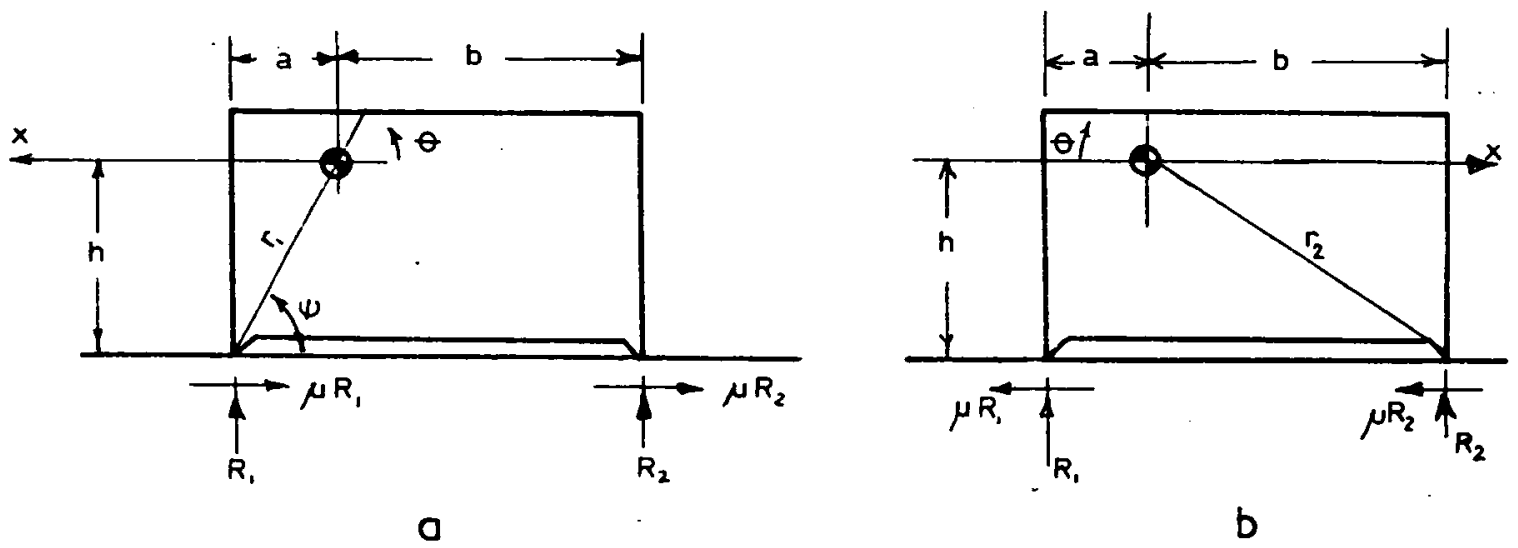

b

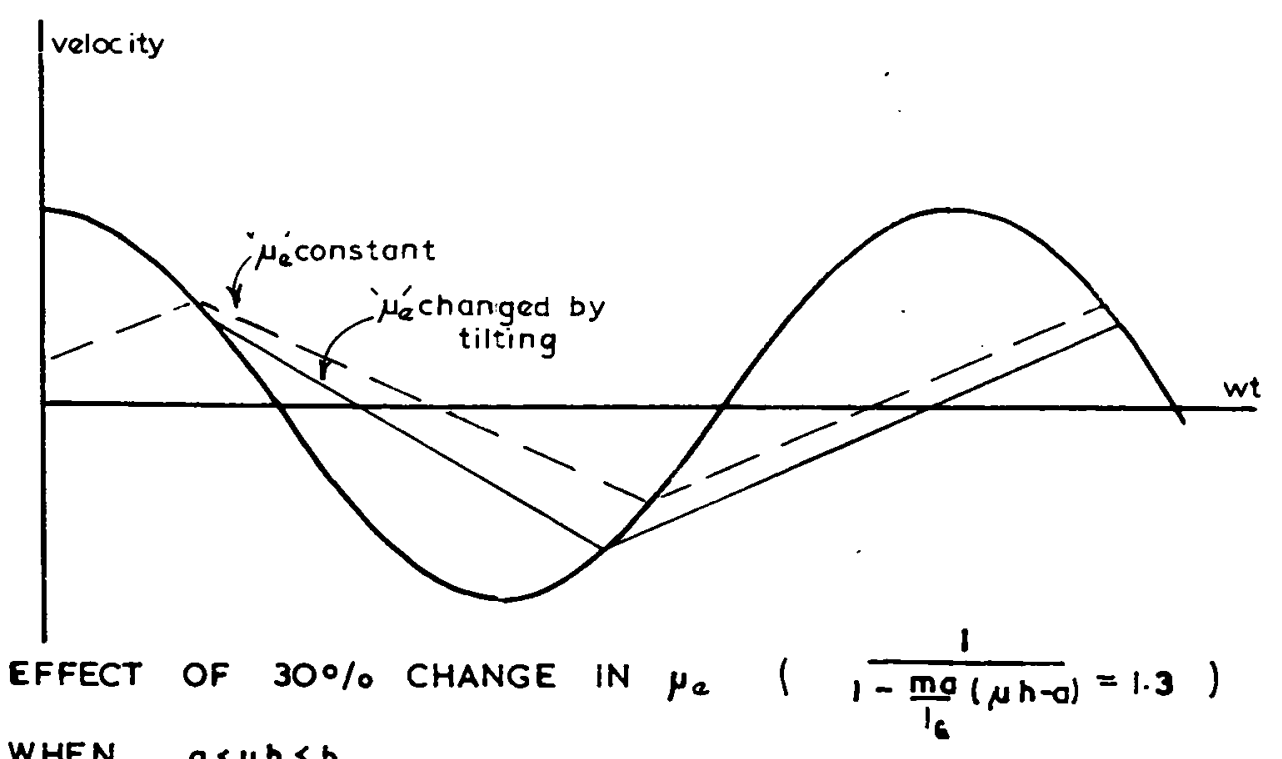

WHEN $a<\mu h<b$

c

FIG $D: 1$ 


$$
\begin{aligned}
\ddot{m} & =-\mu R_{I} \\
\ddot{m} \ddot{y} & =R_{I}-m g \\
I_{G} \ddot{\theta} & =\mu R_{I} h-R_{I} a
\end{aligned}
$$

about the Centre of Gravity.

... D.3

Since the body is pivoting about $A, y=r_{1} \theta \cos \phi=a \theta$ and equations D. 3 give

$$
\begin{aligned}
& \ddot{m}=-\mu R_{I} \\
& R_{I}=m(g+a \ddot{\theta})=\frac{m g}{I-\frac{m a}{I_{G}}(\mu h-a)} \\
& \ddot{x}=\frac{-\mu R_{I}}{m}=\frac{-\mu g}{1-\frac{m a}{I_{G}}(\mu h-a)}
\end{aligned}
$$

where $\mu \mathrm{h} \geq \mathrm{a}$.

Considering now the configuration of Fig. D.1b. When slipping is taking place

$$
R_{1}=\frac{m g(b-\mu h)}{a+b} ; \quad R_{2}=\frac{m g(a+\mu h)}{a+b}
$$

where, as before, $R_{1} \geqslant 0 ; R_{2} \geqslant 0$ and $R_{1}+R_{2}=m g$. The condition at which tilting will begin is $\mu \mathrm{h} \geqslant \mathrm{b}$. Since $\mathrm{b}>\mathrm{a}$ it is likely that 'directional preference' will be caused by tipping in one direction only $\mathrm{a}<\mu \mathrm{h}<\mathrm{b}$. However, any tall, narrow based object may rock symmetrically and seriously effect the mean travel rate. Allowing tilting to take place during the slipping motion gives

$$
\begin{aligned}
m \ddot{x} & =-\mu R_{2} \\
\ddot{m} \ddot{y} & =R_{2}-m g \\
I_{G} \ddot{\theta} & =-b R_{2}+\mu R_{2} h
\end{aligned}
$$

giving

$$
R_{2}=\frac{m g}{1+\frac{m b}{I_{G}}(b-\mu h)}
$$


and

$$
\ddot{x}=\frac{-\mu g}{1-\frac{m b}{I_{G}}(\mu h-b)}
$$

where $\mu \mathrm{h} \geqslant \mathrm{b}$.

If, for example, $a<\mu h<b$, equation $D .4$ shows that a rather greater deceleration of the slider occurs in one direction than in the other. This effect might be thought small, but Fig. D.Ic shows a case when $\frac{1}{1-\frac{m a}{I_{G}}(\mu h-a)}=1.3$ with $\mu=\left(\frac{2}{T_{0}} \cdot \frac{\hat{x} \omega}{g}\right)$, and obviously a fairly fast travel rate will result, the direction of travel being such that the eccentricity of the centre of gravity is diametrically opposite the direction of advance.

The above equations assiume that the rotation is small. If the rotation is allowed to become large more complicated equations result. As before

$$
\begin{aligned}
\ddot{m} \ddot{x} & =-\mu R_{I} \\
m \ddot{y} & =R_{I}-m g \\
I_{G} \ddot{\theta} & =\mu R_{I} h / \cos \theta-R_{I} h \tan (\sigma-\theta)
\end{aligned}
$$

where

$$
y=r_{1} \theta \tan (\sigma+\theta)=\theta(a-h \theta)
$$

Then the equations become

$$
\begin{aligned}
\ddot{m} \dot{x} & =-\mu R_{I} \\
R_{I} & =m g+m\left(a \ddot{\theta}-2 h\left(\dot{\theta}^{2}-\theta \ddot{\theta}\right)\right) \\
I_{G} \ddot{\theta} & =\mu R_{I} h-R_{I} h\left(\frac{a-h \theta}{h+a \theta}\right)
\end{aligned}
$$$$
\text { ... D.9 }
$$

which gives

$$
\begin{aligned}
{[\operatorname{mah}(\mu h-a)-} & I_{G} h+\left\{\operatorname{mh}(2 h \cdot(\mu h-a)+(\mu a+h) \cdot a)-I_{G} h j \theta\right] \ddot{\theta} \\
& -2 m^{2}(\mu h-a+(\mu a+h) \theta) \dot{\theta}^{2} \\
& +\operatorname{mgh}(\mu a+h) \theta+\operatorname{mgh}(\mu h-a)=0
\end{aligned}
$$


The rocking motion of the slider is particularly complicated. Simple experiments indicate modes when the rocking of the slider is periodic in as many as 4 cycles of table motion. The impact at the end of each rock of the slider further complicates the problem. Static considerations show that instability will occur if $\theta>\tan ^{-1}\left(\frac{a}{h}\right)$. However there is no reason to suppose that dynamic instability need occur if this Iimit is exceeded.

Other phenomona which have been noted include the possible reversal of direction of relative slip between the table and slider under certain conditions of frequency and amplitude. On a similar subject it was noticed that when the conveyor surface motion was non-linear a tall thin cylinder on its end had two stable modes of transport. In one it proceeded smoothly with no rock in a direction towards the non-linear 'impact' spring. In the other it rocked violently and moved slowly away from the impact spring. The solution to this may emerge from a study of the complete equations.

In general it may be stated that if a body being transported has a configuration in which it is statically stable such that the distance from its outermost contact point from a perpendicular line through the centre of gravity is less than $\mu \mathrm{h}$ where $\mathrm{h}$ is the height of the centre of gravity above the conveyor surface, then erratic results may occur. 


\section{APPENDTX E}

The complex regions of motion which resulted in the small broken regions of travel rate in Figs. $6.4-6.6$ are very difficult to explain. No system which gave similar results has been found in a search of references.

The problem is complicated by the extremely non-linear nature of the spring characteristic. Control systems with highly non-linear elements are not unusual (9), but most vibration studies are primarily for quasi-linear systems. Hayashi ${ }^{(12)}$ studied harmonic and subharmonic response of a system whose spring force is $K(x)=k_{1} x+k_{2} x^{2}+k_{3} x^{3}$ and using Hill's equation investigates the stability of the solution found. Minorsky ${ }^{(2 / 4)}$ included a chapter on piecewise linearisation of non-linear systems. Methods by Den Hartog(11), Rauscher ${ }^{(29)}$, Brock ${ }^{(6)}$ and Inglis $(14)$, while being capable of solving markedly non-linear equations assume the solution at the outact. The problem of establishing the stability of a solution is not considered and is in fact a major difficulty. To obtain any indication of the behaviour of System A it was found necessary to draw conclusions from the behaviour of quasi-linear systems which have been investigated in detail by many authors.

In chapter 2 the various types of non-linear response were described. The definitions used vere taken from Stoker ${ }^{(35)}$ and Levenson (18). These authors define the solutions in terms of the response of a linear system to forced vibrations and allow the extension of these definitions to non-linear problems. The definitions will be restated. 
Considering the equation $\ddot{x}+p^{2} x=F \cos \omega t \quad$... E.I with initial conditions $\dot{x}(0)=0 ; \quad x(0)=A$ then the solution of $\mathrm{T} .1$ is

$$
x(t)=\left(A+\frac{F}{\omega^{2}-p^{2}}\right) \cos p t-\frac{F}{\omega^{2}-p^{2}} \cos \omega t \quad \ldots \text { E. } 2
$$

The solution $x(t)$ is periodic only in the following cases

a) $\quad A+\frac{F}{\omega^{2}-p^{2}}=0 \quad$ no free oscillations

b) $\quad \omega=n p \quad n>1$

c) $\quad \omega=\frac{1}{m} \cdot p \quad m>1$

d) $\quad \omega=\frac{n}{m} \cdot p \quad m>1, n>1, m$ and $n$ relatively prime

These solutions are defired as a) harmonic; b) subharmonic; c) ultraharmonic and d) ultra-subharmonic.

The extension of these definitions to non-linear differential equations can cause ambiguities. Considering Duffing's equation with a cubic non-linearity

$$
m \ddot{x}+k_{1} x+k_{3} x^{3}=f \cos \omega t \quad k_{3} \operatorname{small} \quad \ldots E .3
$$

This has a solution

$$
x(t)=a_{1} \cos \omega t+a_{3} \cos 3 \omega t+\ldots \quad \ldots E \cdot 4
$$

This solution is usually referred to as the harmonic solution though the term $a_{3} \cos 3 \omega t$ could be called an ultro-harmonic. Similarly a waveform given by

$$
\begin{gathered}
x(t)=a_{0}+a_{1} \cos \left(\frac{1}{2} \omega t+\phi_{1}\right)+a_{2} \cos \left(\omega t+\phi_{2}\right)+a_{3} \cos \left(\frac{3}{2} \omega t+\phi_{3}\right)+\ldots \\
\ldots \text { E. } 5
\end{gathered}
$$

could either be considered as a $\frac{1}{2}$ subharmonic by considering $a_{1}$, or as an ultra-subharmonic by considering $a_{3}$. When one term is very much larger than the others no difficulty exists, but where both terms are 

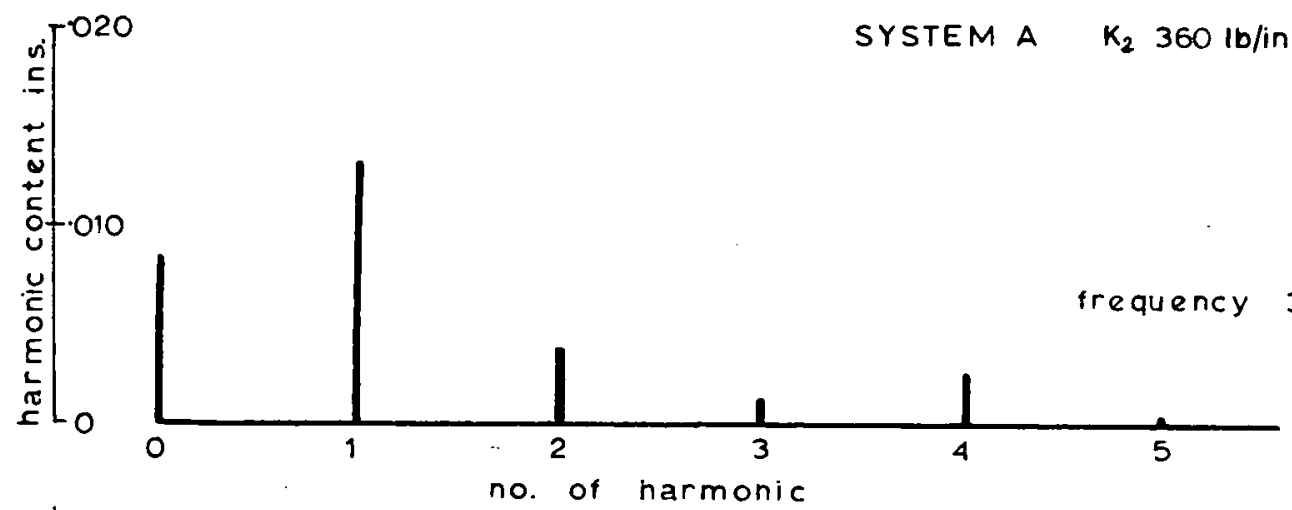

$t_{0}^{1.010}$

no. of harmonic

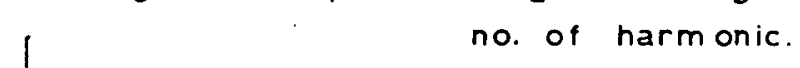

frequency $3.5 \mathrm{c} / \mathrm{s}$.
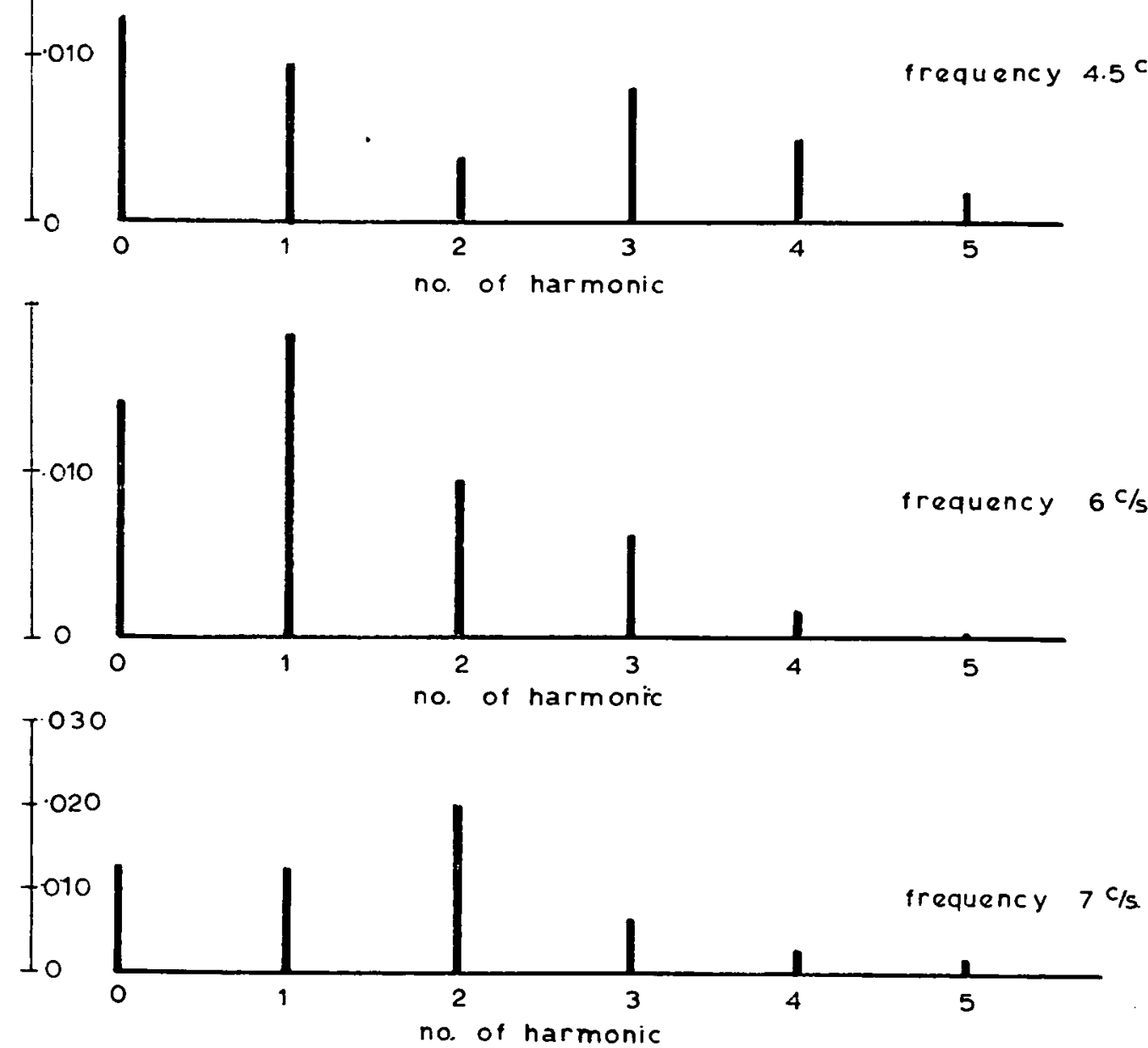

AMPLITUDE OF HARMONIC CONTENT FIG. E:I 


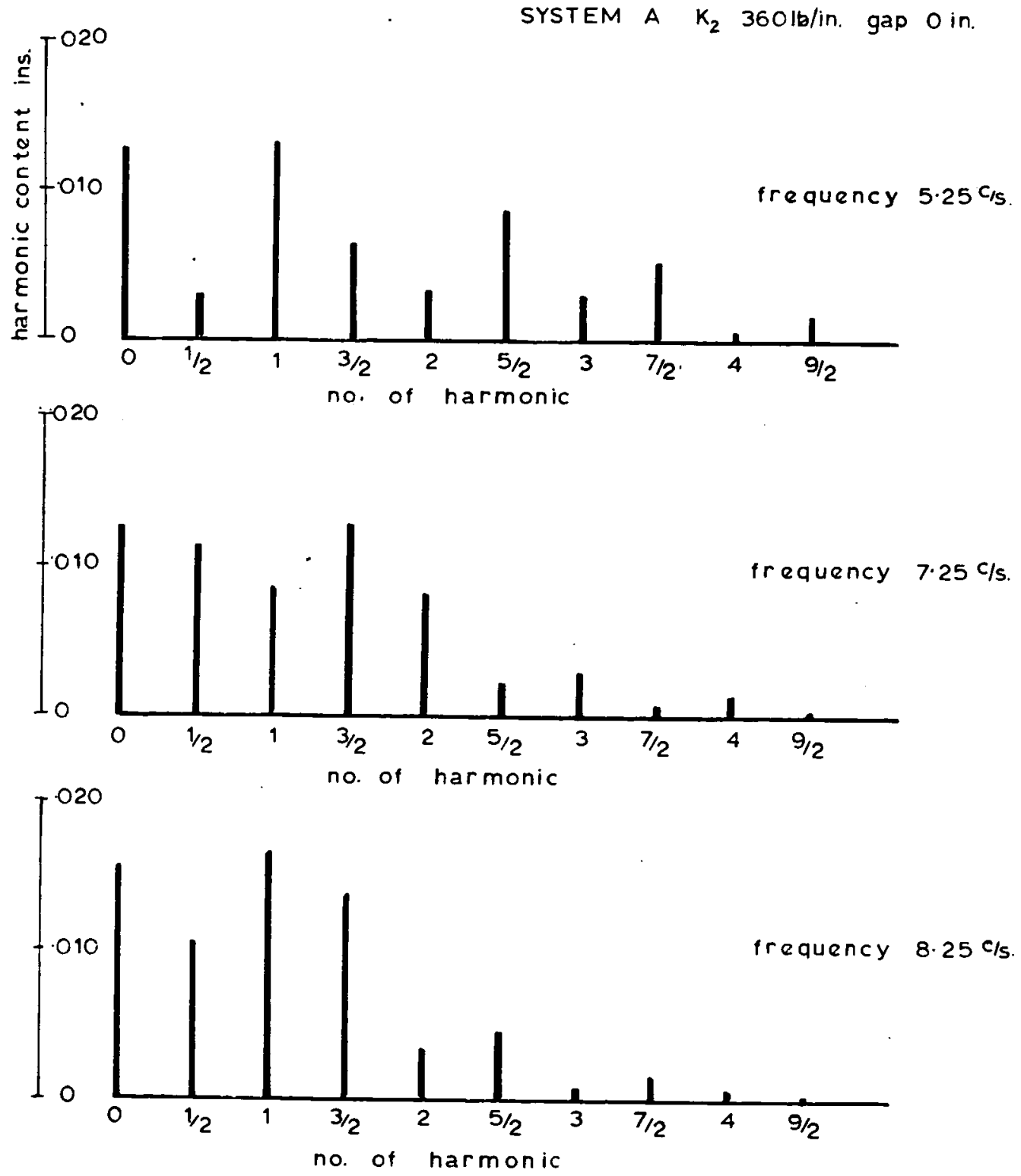

AMPLITUDE OF HARMONIC COMPONENT

FIG E:2 
of the same magnitudes the definition would appear arbitrary. In quasi-linear systems the problem of ultra-harmonic response is seldom considered. Solutions of the type E.5 will not usually emerge from perturbation methods where $x(t)$ is written $x(t)=x_{0}(t)+b x_{1}(t)+b^{2} x_{2}(t)+$ with $b$ small, thus precluding many terms having the same order of amplitude.

Subharmonic response in quasi-linear systems only occurs with the subharmonic component having a frequency close to that of the linear frequency. Subharmonics of the order $\frac{1}{3}$ are obtained from equation $\mathbf{E} \cdot 3$ only in the region $\omega>3 \sqrt{\frac{\mathrm{k} l}{\mathrm{~m}}} \quad$ (23). The equation $m \ddot{x}+k_{1} x+k_{2} x^{2}=F \cos \omega t \quad k_{2} \operatorname{small} \quad \ldots \mathrm{E} .6$ can have regions of subharmonic response both above and below $\omega=2 \sqrt{\frac{k}{m}}$, but the region is closely associated with $\omega=2 \sqrt{\frac{k_{l}}{m}}$. Levenson $(18,19)$ states that similar relationships hold for ultra-subharmonic and ultraharmonic response.

Few of the experimental waveforms obtained had any distinction which would have enabled them to be lettered as ultra-harmonic or ultrasubharmonic response, and early attempts at theoretical solution did not consider these possibilities. Much time was devoted to the method of Hayashi, but no result could be obtained for sub-harmonic response at the frequencies at which it existed experimentally. It was only after repeated attempts had produced no results that it was decided to check the waveforms by harmonic analysis. A simple digital computer progranme was written to read in any number of equally spaced ordinates and to print out the amplitudes of the constant term and the first 9 harmonics, giving the modulus and phase angle. Figs. E.1 and E.2 show the harmonic content from the analysis of several waveforms obtained from System $A$ with $k_{2}=360 \mathrm{lb} / \mathrm{in}$. and zero gap. 
full response

(conjectured)

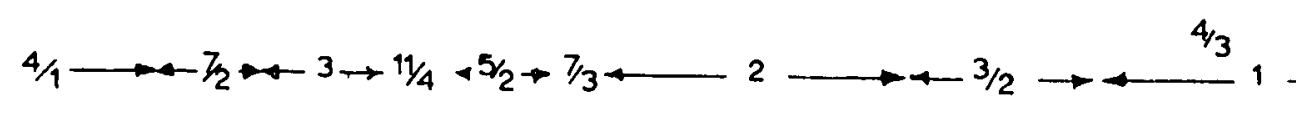

harmonic analysis

of digital comp.

$4 / 1$

$3 / 1 \quad 5 / 2 \quad 7 / 3$

$2 / 1$

$3 / 2$

$1 / 1$

results.

designation on graph 6:4

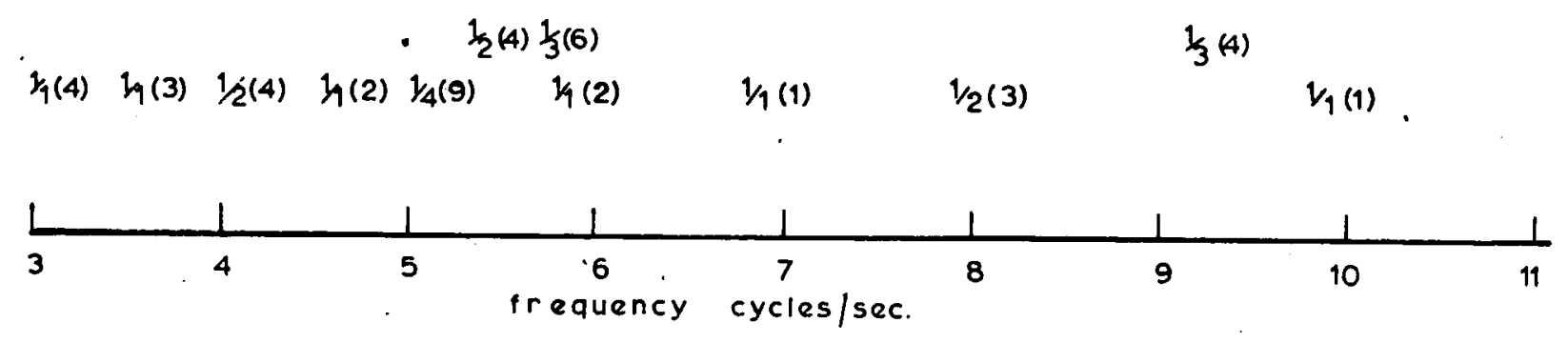

REGIONS OF ULTRA HARMONIC \& ULTRA SUBMARMONIC RESPONSE

FIG E:3 


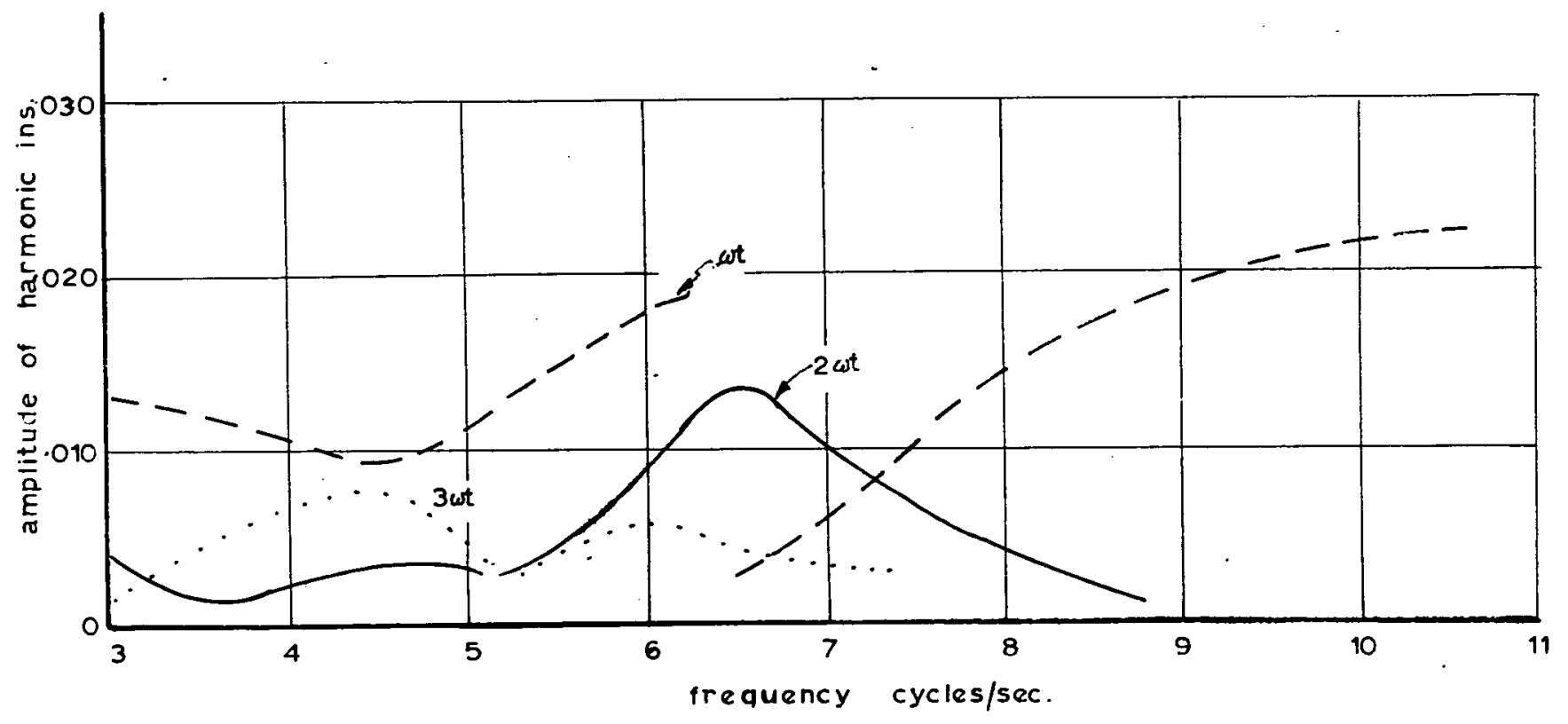

AMPLITUDE OF HARMONIC COMPONENTS

FIG. E:4. 
The trend which it was hoped to establish may best be seen by reference to the analysis of the response at $7 \mathrm{c} / \mathrm{s}$ (Fig. E.I) when the second harmonic is the largest component. This response, it was felt, could justifiably be labelled an ultra-harmonic of order 2 . The response at $6 \mathrm{c} / \mathrm{s}$ would appear to have the characteristics of a true harmonic, but the response at $4.5 \mathrm{c} / \mathrm{s}$ indicates an uItra-harmonic solution of order 3. It can be seen that the harmonic content can be confusing. The presence of high order powers in the expansion $K(X)$, the spring force, will produce complicated solutions. Product terms caused when a series solution is raised to higher powers would appear to ensure that if two harmonics occur to a larger extent, then harmonics which are the sum or difference of the 'dominant harmonics' will be large.

Fig. E.2. shows the harmonic analysis of 3 'subharmonic' waveforms. The upper analysis is held to be that of a $5 / 2$ ultra-subharmonic, and the lower analyses those of $3 / 2$ subharmonics.

Fig. E. 3 is an attempt to identify the various regions of travel rate shown on Fig. 6.4 with various ultra-harmonic and ultra-subharnonic solutions. The jump frequency for this configuration was established by analogue corputer to be between $15 \mathrm{c} / \mathrm{s}$ and $16 \mathrm{c} / \mathrm{s}$. It is reasonable to assume that the 'dominant harmonic' shall have a frequency between $15 \mathrm{c} / \mathrm{s}$ and the frequency of the linear resonance at $8-15 \mathrm{c} / \mathrm{s}$. For all the regions shown on Fig. E.3 the 'dominant harnonic' has a frequency between $I I \mathrm{c} / \mathrm{s}$ and $15 \mathrm{c} / \mathrm{s}$. The amplitudes of the harmonic components are plotted against frequency in Fig. E.4, and this shows that the fundamental term is affected by the presence of higher harmonics. The fundamental term is slightly reduced when the third harmonic term is large, and has an amplitude jump associated with the peak response of 
the second harmonic. This indicates that there is some justification for discounting the amplitude of the fundamental when labelling ultraharmonics and ultra-subharmonics since it is obviously bound up with that of other components.

It is usual in examining subharmonic response of quasi-linear systems to find that at frequencies at which the subharmonic occurs the harmonic solution is also stable. In most cases with System $A$ only one stable solution existed at a particular frequency though the $4 / 3$ ultra-subharmonic and the harmonic solution were both stable at $9.26 \mathrm{c} / \mathrm{s}\left(K_{2}=360 \mathrm{lb} /\right.$ in., gap 0 in。 $)$. However Reuter ${ }^{(31)}$ has shown that in the quasi-linear system represented by

$$
\ddot{x}+K(\dot{X})+\omega^{2} x(1+\alpha x)=f \operatorname{cospt} \quad \ldots . . \text { E. }
$$

relationships can be calculated for frequencies at which there may be two stable solutions or only one, the presence of a subharmonic being able to make the harmonic solution unstable. While in the experiments conducted there were frequancies at which no stable harmonic was found this does not prove that this type of solution did not exist, though by analogy with the work of Reuter this may well be the case.

In one case where two solutions existed (Systen A, gap .025 ins $\mathrm{K}_{2}=360 \mathrm{lb} / \mathrm{in}$. , frequency $7 \mathrm{c} / \mathrm{s}$ ) harmonic analysis of the solutions showed that the presence of the subharmonic and ultra-subharmonic terms had little effect on the amplitude of the harmonic components. From the maximum amplitude in the harmonic solution the autoperiodic freugency was calculated, this calculation being facilitated by the piecevise linear nature of the spring force. The autoperiodic frequency thus obtained was $9.8 \mathrm{c} / \mathrm{s}$ compared with $10.5 \mathrm{c} / \mathrm{s}$ for the $3 / 2$ ultra-subharnonic component. This supports the general relationship that ultra-harmonic of ultra-subharmonic response may occur with the frequency of the 
'dominant harmonic' is close to the auto-periodic frequency calculated from the anplitude of harmonic response at the exciting frequency. However it is not always possible to calculate a meaningful auto-periodic frequency, and also the stability of the solutions of the differential equation is very dependent on damping.

The analytical approach to the problem by the standard techniques of non-linear vibrations requires that the spring force be represented as a power series. Reasonable approximation to the bilinear characteristic used in this thesis required a series expansion to the fifth lower of displacement.

Having established the differential equation with the power series for the restoring spring force no theoretical difficulty exists in applying the Ritz-Galerkin method. A series solution is assumed and inserted onto the differential equation. By multiplying by sinn $t$ and $\cos n t$ and integrating over a cycle a number of simultaneous equations equal to the number of unknowns in the assumed series solution are obtained. The practical problem is to solve these equations as can be seen in the following example with the differential equation

$$
\begin{aligned}
& m \ddot{x}+k_{1} x+k_{2} x^{2}=F \cos 2 \omega t \\
& \text { or } \quad \ddot{x}+p_{1}^{2} x+p_{2}^{2} x^{2}=f \cos 2 \omega t
\end{aligned}
$$

Assume a solution of the form

$$
x=a_{0}+a_{1} \cos \omega t+a_{2} \sin \omega t+a_{3} \cos 2 \omega t+a_{4} \cos 3 \omega t+a_{5} \sin 3 \omega t \ldots \text { E.10 }
$$

Substituting into E.9 and equating coefficients gives

$$
\begin{aligned}
& a_{0} p_{1}^{2}+p_{2}^{2}\left(a_{0}^{2}+\frac{1}{2}\left(a_{1}^{2}+a_{2}^{2}+a_{3}^{2}+a_{4}^{2}+a_{5}^{2}\right)\right)=0 \\
& \left(p_{1}^{2}-{ }^{2}\right) a_{1}+p_{2}^{2}\left(2 a_{0} a_{1}+a_{1} a_{3}+a_{3} a_{4}\right)=0 \\
& \left(p_{1}^{2}-2\right) a_{2}+p_{2}^{2}\left(2 a_{0} a_{3}-a_{2} a_{3}+a_{3} a_{5}\right)=0
\end{aligned}
$$




$$
\begin{aligned}
& \left(p_{1}^{2}-4^{2}\right) a_{3}+p_{2}^{2}\left(2 a_{0} a_{3}+a_{1} a_{4}+\frac{1}{2}\left(a_{1}^{2}-a_{2}^{2}\right)+a_{2} a_{5}\right)=0 \\
& \left(p_{1}^{2}-9^{2}\right) a_{4}+p_{2}^{2}\left(2 a_{0} a_{4}+a_{1} a_{3}\right)=0 \\
& \left(p_{1}^{2}-9^{2}\right) a_{5}+p_{2}^{2}\left(2 a_{0} a_{5}+a_{2} a_{3}\right)=0
\end{aligned}
$$

Even for this simple example solution of these equations presents a very large problem. Without cohsidering a larger series solution for the displacement, if the spring constant contains higher powers of $\mathrm{x}$ the calculation becomes excessive. While digital computer methods could possibly be developed to solve these equations this would destroy any benefit that an analytic approach might have. A disadvantage of the above method is that it would require to be repeated for all expected subharmonics, and having established a solution at a given frequency its stability would have to be checked. Hayashi (12) used this approach for simple subharmonic response and investigated the stability via the variational equation and the stability regions of Hill's equation.

The perturbation method used by Levenson ${ }^{(18)}$ and Reuter ${ }^{(31)}$ is essentially a quasi--Iinear technique. Starting from the equation

$$
\ddot{y}+a y+b y^{2}=f \cos 2 \omega t
$$

$\omega t$ is put equal to $z$. The small perturbation can be arbitrarily chosen but $\mathrm{b}$ is generally used. It is convenient, though not strictly necessary to put $f=b F$ and assume that the force is amall with the perturbation factor. This approach was tried for a $3 / 2$ ultra-subharmonic but in the course of the procedure gave the solution that the ultra-subharmonic was zero. Leaving the force as $f$ gives

$$
\omega^{2} \ddot{y}+a y+b y^{2}=f \cos 2 z
$$


with

$$
\begin{aligned}
& y=y_{0}+b y_{1}+b^{2} y_{2}+. \\
& \omega=\omega_{0}+b \omega_{1}+b^{2} \omega_{2}+.
\end{aligned}
$$

substituting $\$ .14$ in E.13 gives

$$
\begin{array}{r}
\left(\omega_{0}^{2}+2 b \omega_{0} \omega_{I}+b^{2}\left(\omega_{1}+2 \omega_{0} \omega_{2}\right)+\ldots\right)\left(\ddot{y}_{0}+b \ddot{y}_{I}+b^{2} \ddot{y}_{2}+\ldots\right) \\
+a\left(y_{0}+b y_{I}+b^{2} y_{2}+\ldots\right)+b\left(y_{0}^{2}+2 b y_{0} y_{I}+\ldots\right)=f \cos 2 z \\
\ldots . . .15
\end{array}
$$

Equating coefficients of $b^{r}, r=0,1,2 \ldots$ on each side of E.15 yields $\mathrm{b}^{\mathrm{O}}$ :

$$
\begin{aligned}
& \omega_{0}^{2} \ddot{y}_{0}+a y_{0}=f \cos 2 z \\
& y_{0}=A \cos \sqrt{\frac{a}{\omega_{0}}} \cdot z+B \sin \sqrt{\frac{a}{\omega_{0}}} \cdot z-\frac{f}{4 \omega^{2}-a} \cos 2 z \quad \ldots \pm .16
\end{aligned}
$$

where $A$ and $B$ are established by initial conditions. In order that the solution $\mathrm{k} .16$ should be periodic in $2 \pi, \frac{\sqrt{a}}{\omega_{0}}$ must be an integer. If $V_{a}=3 \omega_{0}$ then

$$
y_{0}=A \cos 3 z+B \sin 3 z+\frac{f}{5 \omega_{0}^{2}} \cos 2 z
$$

$b^{1}:$

$$
\omega_{0}^{2} \ddot{y}_{1}+a y_{1}=-y_{0}^{2}-2 \omega \omega_{1} \ddot{y}_{0}
$$

Substituting for $y_{0}$ and removing secular terms gives $\omega_{I}=0$ and $y_{1}$ has a solution of the form

$$
\begin{array}{r}
y_{1}=\alpha+\beta \cos z+\gamma \sin z+\delta \cos 4 z+\epsilon \cos 5 z+\rho \sin 5 z+\sigma \sin 6 z+\not \phi \cos 6 z \\
\ldots \text { E. } 17
\end{array}
$$

and hence the solution to the first approximation is

$$
y=A \cos 3 z+B \sin 3 z+\frac{f}{5 \omega_{0}^{2}} \cdot \cos 2 z+b y_{I} \quad \ldots . E \cdot I 8
$$

Continuing to the coefficient of $b^{2}$ gives

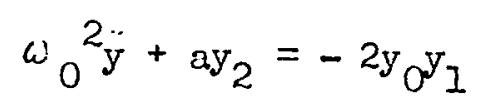

By removing secular terms, equations may be obtained which allow the calculation of $\left(A^{2}+B^{2}\right)$ and $\omega_{2}$. 
The above approach is similar to that used by Levenson, and can be seen to be of linited practical interest, for damping which is known to have an important effect on subharmonic response has been neglected.

A reference to ultra-subharmonic response has been found in combination tones in non-linear systems ${ }^{(8)}$, again using Duffing's equation with a cubic non-linearity. Further references on subharmonic response and other topics are listed in the Bibliography. Bearing in mind the dominance of these regions of uItra-harmonic and ultrasubharmonic response it is surprising that they are not mentioned in other references. Thay were not reported by Stephen ${ }^{(34)}$ who used a similar system with a rigid stop, and who only describes a limited class of pure subharmonic. It is known that in System $A$ of this thesis the results with a rigid stop were similar to those listed in Fig. E.3. 